\title{
REAL-TIME COMPUTER PLATFORM FOR VIBRATION-BASED \\ STRUCTURAL HEALTH MONITORING OF THE \\ CONFEDERATION BRIDGE
}

\author{
Submitted by \\ Serge L. Desjardins, B.Sc.A. \\ Université de Moncton, N.-B.,Canada
}

A thesis submitted to the Faculty of Graduate Studies and Research in partial fulfillment of the requirement for the degree of Master of Applied Science

\author{
Department of Civil and Environmental Engineering \\ Carleton University \\ Ottawa, Ontario
}

도 Serge L. Desjardins, 2004

The Master of Applied Science in Civil Engineering is a joint program with the University of Ottawa, administered by the Ottawa-Carleton Institute of Civil Engineering 


$\begin{array}{ll}\begin{array}{l}\text { Library and } \\ \text { Archives Canada }\end{array} & \begin{array}{l}\text { Bibliothèque et } \\ \text { Archives Canada }\end{array} \\ \begin{array}{l}\text { Published Heritage } \\ \text { Branch }\end{array} & \begin{array}{l}\text { Direction du } \\ \text { Patrimoine de l'édition }\end{array} \\ \begin{array}{l}\text { 395 Wellington Street } \\ \text { Ottawa ON K1A 0N4 }\end{array} & \begin{array}{l}\text { 395, rue Wellington } \\ \text { Ottana ON K1A ON4 } \\ \text { Canada Oa }\end{array}\end{array}$

Your file Votre référence ISBN: 0-494-00737-0

Our file Notre référence

ISBN: 0-494-00737-0

NOTICE:

The author has granted a nonexclusive license allowing Library and Archives Canada to reproduce, publish, archive, preserve, conserve, communicate to the public by telecommunication or on the Internet, loan, distribute and sell theses worldwide, for commercial or noncommercial purposes, in microform, paper, electronic and/or any other formats.

The author retains copyright ownership and moral rights in this thesis. Neither the thesis nor substantial extracts from it may be printed or otherwise reproduced without the author's permission.
AVIS:

L'auteur a accordé une licence non exclusive permettant à la Bibliothèque et Archives Canada de reproduire, publier, archiver, sauvegarder, conserver, transmettre au public par télécommunication ou par l'Internet, prêter, distribuer et vendre des thèses partout dans le monde, à des fins commerciales ou autres, sur support microforme, papier, électronique et/ou autres formats.

L'auteur conserve la propriété du droit d'auteur et des droits moraux qui protège cette thèse. $\mathrm{Ni}$ la thèse ni des extraits substantiels de celle-ci ne doivent être imprimés ou autrement reproduits sans son autorisation.
In compliance with the Canadian

Privacy Act some supporting forms may have been removed from this thesis.

While these forms may be included in the document page count, their removal does not represent any loss of content from the thesis.
Conformément à la loi canadienne sur la protection de la vie privée, quelques formulaires secondaires ont été enlevés de cette thèse.

Bien que ces formulaires aient inclus dans la pagination, il n'y aura aucun contenu manquant. 


\section{Abstract}

Structural Health Monitoring (SHM) is the in-depth analysis of structural wellbeing by using various field measured data. Recently, vibration-based structural health monitoring (VBSHM) techniques are attracting great interest for its adapting to civil engineering applications, where sensor captured vibration responses of the monitored structure due to ambient environmental loading, such as wind and traffic, are used to assess the condition of the structure. The VBSHM technique is based on the premise that changes in vibration characteristics of a physical system reflects potential deterioration or damage in the system. It can overcome the difficulties and limitations of conventional structural evaluation techniques, such as acoustic, ultra-sonic and magnetic field techniques, which need a priori knowledge of the potential damage location and can only provide localized information on the condition of the structure. The Confederation Bridge monitoring project at Carleton University offers a unique opportunity to advance the technologies and the state-of-the-art of the emerging new field. Adapting the VBSHM technique to continuous monitoring of large complex civil engineering structures results in the need to process and analyze large amount of monitoring data to extract the vibration characteristics of the system. It is obvious there is a very practical need for the development of advanced integrated computer tools to automate this complex process. The thesis concerns the development of such an important integrated computer platform, which greatly accelerates and streamlines the processing and analysis of data to extract important engineering information. The development of the modular platform with user friendly graphical user interface (GUI) not only represents major advances in numerical 
techniques for VBSHM, it is also an achievement in transfer of technology from research to engineering practice by providing bridge engineers and operators the tools they need for real-time operating and maintenance decision support. The integrated computer platform includes separate modules for data-management, signal processing, data analysis, system identification, and visualization. The analysis module employs a new highly robust and accurate output-only stochastic subspace system identification technique, developed and implemented by the research team at Carleton University, to obtain the dynamic properties of the structure (frequencies, mode-shapes and damping ratios). The visualization module, which includes a detailed three-dimensioned model of the bridge, can animate any field observed behaviour from the monitoring data in realtime or can animate extracted information, such as vibration mode-shapes. The visualization engine also permits rapid viewing and comparison of behaviour patterns of the monitored structure and thus gives important engineering information needed for decision support to end-users of the structure.

Major research has been carried out by the research team at Carleton University using these advanced modules and tools, such as verification of the design assumptions of the Confederation Bridge. A very good correlation is obtained between the field measured vibration properties of the structure and those based on calibrated finite element models of the bridge. Through extensive detailed analysis of the dynamic monitoring data, vibration signature characteristics of the bridge have been established, despite the uncertainties present at the bridge site resulting in high noise in the data signals. For the first time ever, a relationship between variability in the field measured vibration characteristics and temperature changes of the bridge is discovered and quantified. 
Je dédie cet ouvrage

à Véronik, Jean-Marie, Lorraine et Mélanie.

Je vous aime. 


\section{Acknowledgements}

I would like to express most sincere gratitude to Professor David T. Lau, for his time, expertise and enthusiasm throughout this research. It has been a great honor for me to work and learn from this great knowledgeable person and internationally renowned academic researcher.

Financial support for this research from the Natural Science and Engineering Research Council of Canada (NSERC) in the form of a post-graduate scholarship is gratefully acknowledged. Additional funding from Carleton University and its Faculty of Graduate Studies and Research is also gratefully acknowledged.

Special thanks are owed to Professor Heng A. Khoo for his considerations and input, to Don McGinn of Strait Crossing Bridge Ltd. (SCBL) for his suggestions and hospitality and to John Eagan of Public Works and Government Services Canada (PWGSC) for his help and expertise with maintenance of the data acquisition system.

Most importantly to my dear friend and colleague, Nicolàs Londoño, for his work, diligence, tremendous help, knowledge, co-operation, encouragement and patience, I owe my greatest and sincerest thanks.

I wish to take this opportunity to express my deepest appreciations to my family and extended family for their love, encouragement and motivation without which this work would not have been possible. 


\section{Table of Contents}

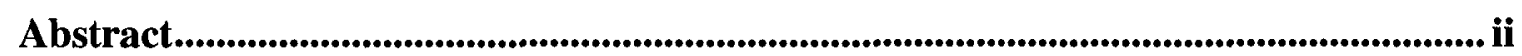

Acknowledgements .......................................................................................................................... v

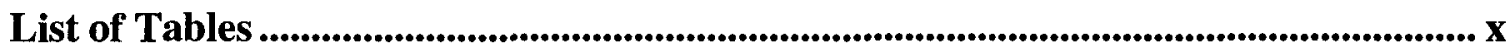

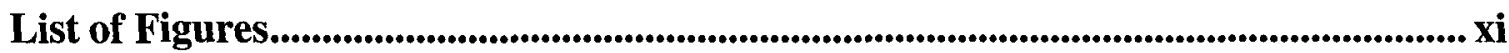

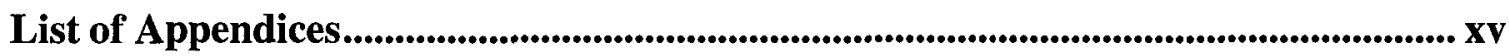

Chapter 1 - Introduction ............................................................................................................................... 1

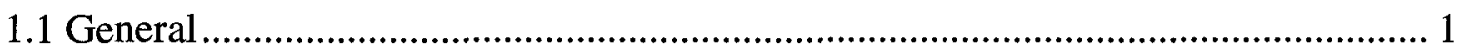

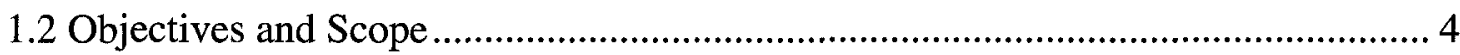

Chapter 2 - Vibration-Based Structural Health Monitoring...............................................9

2.1 Sensing Technologies .................................................................................... 12

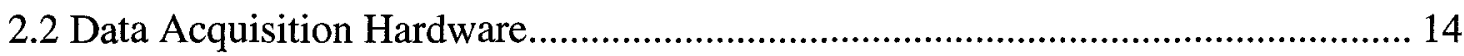

2.3 High-Speed Communication Network.............................................................. 17

2.4 Data Processing, Analysis \& Condition Assessment................................................ 18

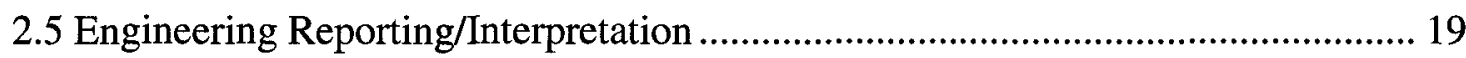

2.6 International Vibration-Based Field Monitoring Projects ........................................ 20

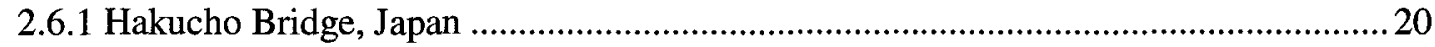

2.6.2 Vasco de Gama Bridge, Portugal .........................................................................2

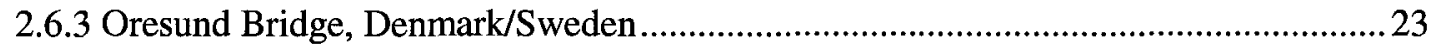

2.6.4 Runyang Yangtze River Bridge, China......................................................................2 24 


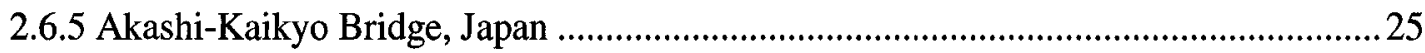

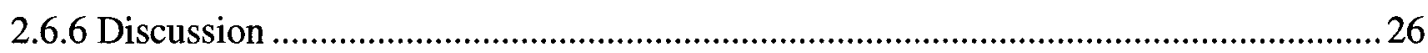

Chapter 3 - Monitoring of the Confederation Bridge................................................. 30

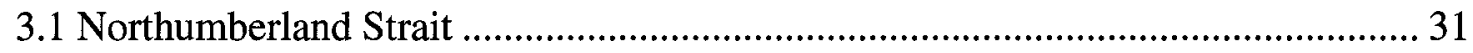

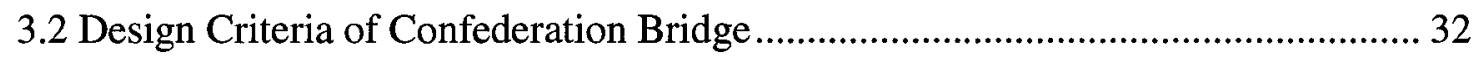

3.3 Structural Layout of Confederation Bridge ...................................................... 32

3.4 Confederation Bridge Monitoring Project ...................................................... 33

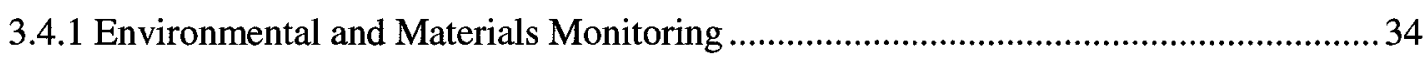

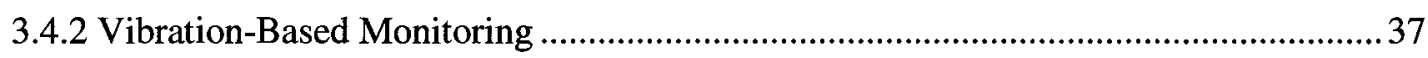

Chapter 4 - Processing of Dynamic Monitoring Data .............................................. 48

4.1 Dynamic Vibration Monitoring Data.............................................................. 50

4.2 Data Management and Pre-processing Operations ........................................... 53

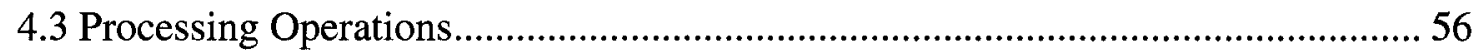

4.4 Processing Module with Graphical User Interface (GUI) .................................... 61

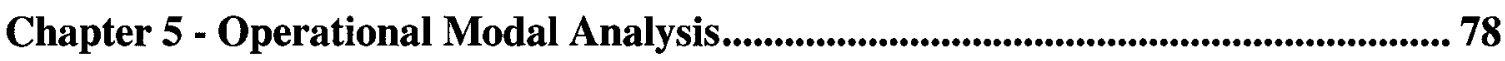

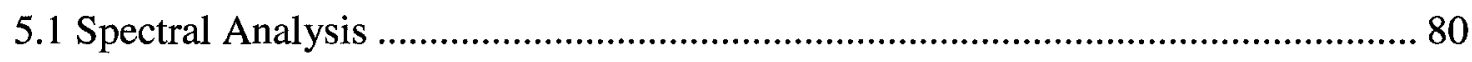

5.1.1 Welch's Power Spectral Density Estimate ............................................................. 81

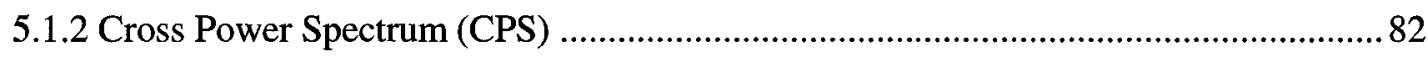

5.2 Stochastic Subspace Identification Method ................................................... 83

5.3 Analysis Modules with Graphical User Interface ............................................ 89

Chapter 6 - Real-Time Visualization of Monitoring Data .................................... 105

6.1 Data Display Module with Graphical User Interface .................................... 106 


\section{Chapter 7 - Applications of Real-Time Structural Health Monitoring Computer}

Platform for Dynamic Research on the Confederation Bridge ................................. 118

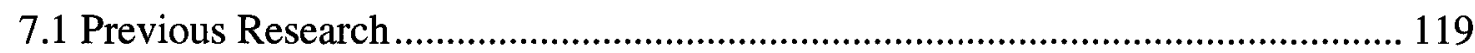

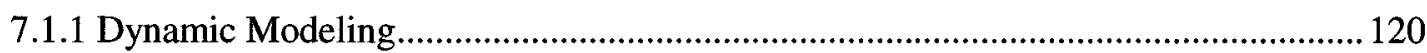

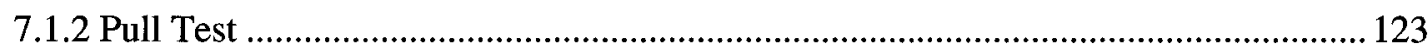

7.1.3 Dynamic Characteristics Obtained From Ambient Excitations ................................ 124

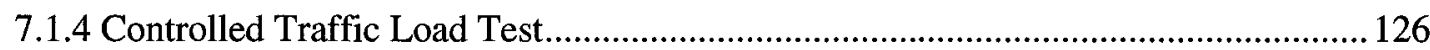

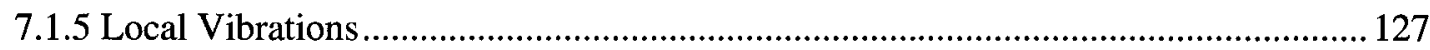

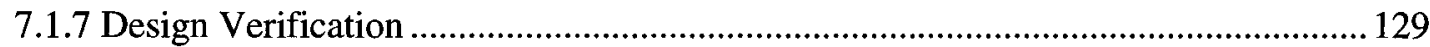

7.1.8 Baseline Variability of Modal Properties.................................................................. 131

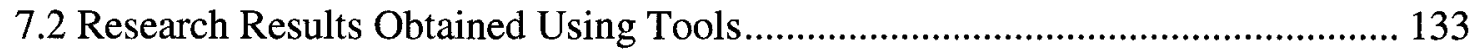

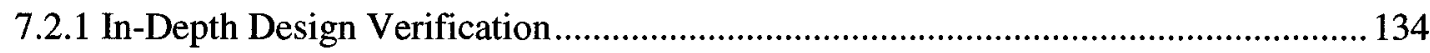

7.2.2 Observed Behaviour of the Confederation Bridge .................................................. 138

7.2.3 Environmental Effects on the Variability of Modal Properties...................................139

Chapter 8 - Future Developments and Conclusions ............................................................. 173

8.1 Future Developments and Recommendations ............................................. 173

8.1.1 Further Research into Variability and Damage Identification ................................ 174

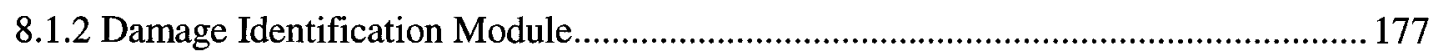

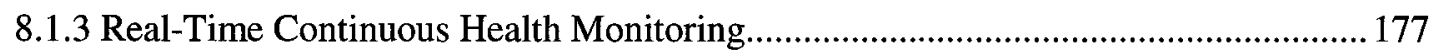

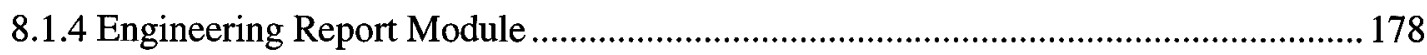

8.2 Conclusion

References........................................................................................................................ 181

Appendix A - Types of Accelerometers ........................................................................... 187

Appendix B - Example of Accelerometer and Data-Logger............................................... 190

Appendix C - Processing Module Code Structure .......................................................... 191 
Appendix D - Analysis Module Code Structure ........................................................ 192

Appendix E - Visualization Modules Code Structure........................................... 193 


\section{List of Tables}

Table 3.1. Sensor location and model of accelerometers 40

Table 3.2. Specifications for accelerometers located on Confederation Bridge. 41

Table 4.1. Typical comma separated data file 64

Table 4.2. Tab-delimitated intermediate data format. 65

Table 7.1. Modal properties extracted by different authors from dynamic monitoring data of the Confederation Bridge. 143

Table 7.2. Verification of experimental modal propeties 144

Table 7.3. Summary of in-depth verification study 145

Table 7.4. Analysis results for study of variability due to environmental effects. ..... 146

Table 7.5. Variability of results from analysis of 42 data sets. 147

Table 7.6. Results of regression analysis in environmental variability study 148 


\section{List of Figures}

Figure 1.1. Screen-capture of SPPLASH modules for Confederation Bridge SHM.... 7

Figure 1.2. Pictures of the Confederation Bridge. 8

Figure 2.1. Pendulum analogy for aliasing problem in sampling. 28

Figure 2.2. Resolution and range of data-logger sampling. 29

Figure 3.1. Location of Confederation Bridge 42

Figure 3.2. Bridge components. 43

Figure 3.3. Ice monitoring instrumentation. 44

Figure 3.4. Creep and shrinkage loading device. 45

Figure 3.5. Accelerometer locations 46

Figure 3.6. Data transmission schematic. 47

Figure 4.1. Typical response monitoring data due to wind excitation. 66

Figure 4.2. Typical response monitoring data due to traffic excitation. 67

Figure 4.3. Typical response monitoring data due to wind excitation (40 seconds)... 68

Figure 4.4. Typical response monitoring data due to traffic excitation (40 seconds).. 69

Figure 4.5. Common data characteristics and corrections. 70

Figure 4.6. Finite Impulse Response (FIR) low-pass filter. 71

Figure 4.7. Infinite Impulse Response (IIR) low-pass filter 72 
Figure 4.8. Example of a decimated signal. 73

Figure 4.9. Example of an un-filtered signal at location $18 \mathrm{~T}$. 74

Figure 4.10. Infinite Impulse Response (IIR) high-pass filter . 75

Figure 4.11. Integration of acceleration and velocity data. 76

Figure 4.12. Processing module. 77

Figure 5.1. Example of a windowed segment of data. .95

Figure 5.2. Power spectral density estimates of signals captured under strong wind.. 96

Figure 5.3. Power spectral density estimates of signals captured during traffic. 97

Figure 5.4. Typical FFT of time history signal 98

Figure 5.5. Typical FFT of windowed time history signal 99

Figure 5.6. Typical auto-power spectrum of a signal. 100

Figure 5.7. Typical stabilization plot showing stability of poles. 101

Figure 5.8. Data display module incorporating Welch's PSD estimate. 102

Figure 5.9. System Identification GUI. 103

Figure 5.10. Assembling of the $\mathbf{Y}_{\text {pref }}$ and $\mathbf{Y}_{f}$ matrices for the block Toeplitz matrix. 104

Figure 6.1. Screen capture of the animation module. 113

Figure 6.2. Simple 3D wire mesh model of instrumented portion of bridge 114

Figure 6.3. Detailed 3D model of instrumented portion of Confederation Bridge.... 115

Figure 6.4. Fine meshed 3D model of the Confederation Bridge 116 
Figure 6.5. Sensor locations and labels shown on the fine meshed 3D model. 117

Figure 7.1. Finite element model using 3D beam elements. 149

Figure 7.2. 3D fine-meshed shell finite element model 150

Figure 7.3. Vertical and transverse acceleration time histories 151

Figure 7.4. Vertical and transverse spectral density estimates 152

Figure 7.5. Mode-shape comparison of $0.34 \mathrm{~Hz}$ mode. 153

Figure 7.6. Mode-shape comparison of $0.37 \mathrm{~Hz}$ mode 154

Figure 7.7. Mode-shape comparison of $0.47 \mathrm{~Hz}$ mode 155

Figure 7.8. Mode-shape comparison of $0.57 \mathrm{~Hz}$ mode 156

Figure 7.9. Mode-shape comparison of $0.65 \mathrm{~Hz}$ mode. 157

Figure 7.10. Mode-shape comparison of $0.68 \mathrm{~Hz}$ mode. 158

Figure 7.11. Mode-shape comparison of $0.92 \mathrm{~Hz}$ mode. 159

Figure 7.12. Mode-shape comparison of $0.97 \mathrm{~Hz}$ mode. 160

Figure 7.13. Mode-shape comparison of $1.32 \mathrm{~Hz}$ mode 161

Figure 7.14. Mode-shape comparison of $1.61 \mathrm{~Hz}$ mode 162

Figure 7.15. Mode-shape comparison of $1.81 \mathrm{~Hz}$ mode 163

Figure 7.16. Mode-shape comparison of $2.75 \mathrm{~Hz}$ mode 164

Figure 7.17. Mode-shape comparison of $3.45 \mathrm{~Hz}$ mode 165

Figure 7.18. Mode-shape comparison of $4.53 \mathrm{~Hz}$ mode 166 
Figure 7.19. Mode-shape comparison of $5.13 \mathrm{~Hz}$ mode.

Figure 7.20. Response frequency behaviour to typical high wind scenario. 168

Figure 7.21. Response frequency behaviour to controlled heavy traffic scenarios. ... 169

Figure 7.22. Response frequency behaviour to typical ambient scenarios with ice ... 170

Figure 7.23. Response frequency behaviour to ambient scenarios with no ice 171

Figure 7.24. Operational modal frequency variation with respect to temperature. ... 172 


\section{List of Appendices}

Appendix A - Types of Accelerometers.................................................................... 187

Appendix B - Example of Accelerometer and Data-Logger......................................... 190

Appendix C - Processing Module Code Structure ………………………………….... 191

Appendix D - Analysis Module Code Structure ...................................................... 192

Appendix E - Visualization Modules Code Structure ................................................... 193 


\section{Chapter 1}

\section{Introduction}

\subsection{General}

Research on vibration-based structural health monitoring (VBSHM) of the Confederation Bridge is presented in this thesis. With recent advances, VBSHM has been attracting increasing interest and research as a very promising method for non-destructive evaluation of civil engineering structures. Sophisticated structural monitoring systems permit capture of motion response data information by means of vibration measurements. From the vibration data, modal properties, such as vibration frequencies, mode shapes and damping ratios, of the structure can be identified. By monitoring the identified dynamic characteristics of the structure, any observed changes in the vibration frequency, mode-shape or damping ratio are considered as manifestations of a possible damage scenario of the structure. Changes in modal characteristics reflect changes in physical 
properties and therefore in the physical conditions of the structure. Difficulties occur in the implementation of VBSHM in the field, where monitoring data are affected by noise and other uncertainties of the environment. The solution to alleviate this problem and facilitate correct interpretation of the engineering information embedded in the data is to establish the trend and detailed behaviour and characteristics of the monitoring data by long-term collection of the information, even over the life span of the facility. For longterm monitoring, structural monitoring systems can be permanently installed on the structure to capture important responses due to extreme events such as earthquakes, hurricanes, ice floes or impacts. The captured monitoring data during extreme events can be used to rapidly evaluate the structure's safety, its performance and conditions. Vibration-based monitoring systems are generally non-intrusive and they do not interfere with the normal function and operation of the monitored structure. In order to extract the needed information from the data acquired from these monitoring systems, highly sophisticated and complex numerical operations for signal processing purposes need to be performed. System identification of modal properties of the structure from the response monitoring data is a central component of VBSHM techniques. It is the basic step in the algorithms for identifying damage in the structure or assessing the structural condition from response vibrations. Structural responses caused by normal operating loads, such as moderate wind or traffic (in the case of bridges), are referred to as ambient responses. These ambient responses are very important for continuous long-term vibration-based structural evaluation techniques. The identification of modal properties, such as frequencies, mode-shapes and damping ratios, from these ambient responses has been shown to be very complicated due to the high level of uncertainties of the input 
excitations, the very low amplitude of the responses, high noise contaminations of the data, non-stationarity of the signals, non-linearity of the structural behaviour resulting in high uncertainty of the signals. Special system identification (SI) algorithms that can effectively reduce the effects of noise contaminations by means of signal correlations have been proven to be the most robust output-only identification techniques. It is only after the successful and consistent identification of modal properties is achieved, that damage identification algorithms can be developed which deal with the variability of the identified dynamic parameters. As of yet no field monitoring project has successfully implemented all these aspects in a complete continuous vibration-based condition evaluation of a large-scale operating civil engineering structure. The ultimate goal of current VBSHM research is to provide continuous evaluation of structural conditions for safety and/or maintenance purposes.

In order to properly capture motions of a physical system, sampling of information needs to be performed at relatively high frequencies; in the order of 100 to $250 \mathrm{~Hz}$ (samples per second). This high sampling frequency combined with long-term monitoring operations produces large amounts of structural response data that needs to be efficiently managed, processed, and analyzed. The sheer amount of collected vibration data shows that the need for a highly sophisticated computer platform for rapid and efficient data management, signal processing, and system identification operations is evident. Presented in this thesis is the development of the Signal processing PLatform for Analysis of Structural Health (SPLASH), a centralized networked computer platform for VBSHM operations. What distinguishes this computer platform from others are the highly sophisticated easy-to-use custom graphical user interfaces (GUI) created for all 
possible vibration-based data operations and interactions (Desjardins et al. 2004) as shown in Figure 1.1. The visualization modules integrated in the platform are the most practical and provide the most useful engineering information. The ability to visualize real-time animation of structural responses is an asset for any structure's engineer and/or operator.

The Confederation Bridge in eastern Canada provides the ideal and unique opportunity for in-depth studies into the use of vibration monitoring data for successful identification of modal properties and subsequent condition assessment by non-destructive means. An elaborate monitoring system has been installed on a representative portion of the $12.9 \mathrm{~km}$ long structure and has been collecting vibration data since 1997. The data collected from the Confederation Bridge has been the subject for the development of the centralized computer platform described in detail in this thesis.

\subsection{Objectives and Scope}

The primary objective of this thesis is to develop a versatile and user friendly computer platform of modules and tools for easy and timely processing and analysis of vibrationbased structural health monitoring (VBSHM) data. The development of the structure and various components and modules of the computer platform is discussed. The computer platform is developed specifically for the Confederation Bridge monitoring project. However, the structure and organization of the platform and computer tools are developed in modular form and are thus project independent, which can be easily adapted 
and modified for monitoring of other infrastructures or facilities. Research results obtained using modules of the computer platform are also presented.

The uniqueness of the Confederation Bridge, shown in Figure 1.2, and the collected monitoring data since its opening offer great opportunities to carry out research on vibration-based structural health monitoring which attempts to assess the health of structures using state-of-the-art signal processing and system identification techniques. Structural Health Monitoring (SHM) is the in-depth analysis of structural wellbeing using different types of field data. Therefore detailed knowledge of all measurable parameters is essential for correct interpretation of the monitoring data to arrive at accurate assessment results of the condition of the evaluated structures. Recent developments and advances in the field of SHM that have received plenty of attention are the techniques and application of vibration-based structural health monitoring (VBSHM) using sensors to capture vibration responses of the monitored structure, which are then used to assess or evaluate the condition or performance of the structure. Field vibration-based monitoring projects often generate large amounts of raw data. The raw data need to be efficiently managed, processed and analyzed. Hence, the need for a VBSHM computer application platform is evident. This thesis concerns the development of this important computer tool and platform which can greatly facilitate and expedite the processing and analysis of the data to extract valuable engineering information. This developed computer platform with the various modules and user interfaces are used to facilitate the continuing research of SHM by analyzing large amounts of monitoring data to determine the actual field dynamic properties of the Confederation Bridge. The behaviour and observed variability of the field extracted dynamic properties are discussed. The visualization tools 
developed to facilitate the interpretation of monitoring data and vibration behaviour of the Confederation Bridge are discussed, which can be used as real-time decision support tools for the operation of the monitored facilities by engineers and operators.

The organization of this thesis is divided into 8 chapters:

Chapter 2 gives an introduction on the motivations and details of VBSHM.

Chapter 3 presents the Confederation Bridge monitoring project.

Chapter 4 deals with the processing of the vibration monitoring data and the development of a specialized real-time module with GUI.

Chapter 5 presents the analysis and system identification operations performed on the vibration monitoring data and the development of the computer modules.

Chapter 6 discusses the real-time visualization and engineering interpretation of the vibration monitoring data.

Chapter 7, in its first part, is a literature review of research on Confederation Bridge vibration monitoring data, while the second part covers research studies performed with the developed tools presented in the thesis.

Chapter 8 discusses future possible developments on the platform with concluding remarks. 


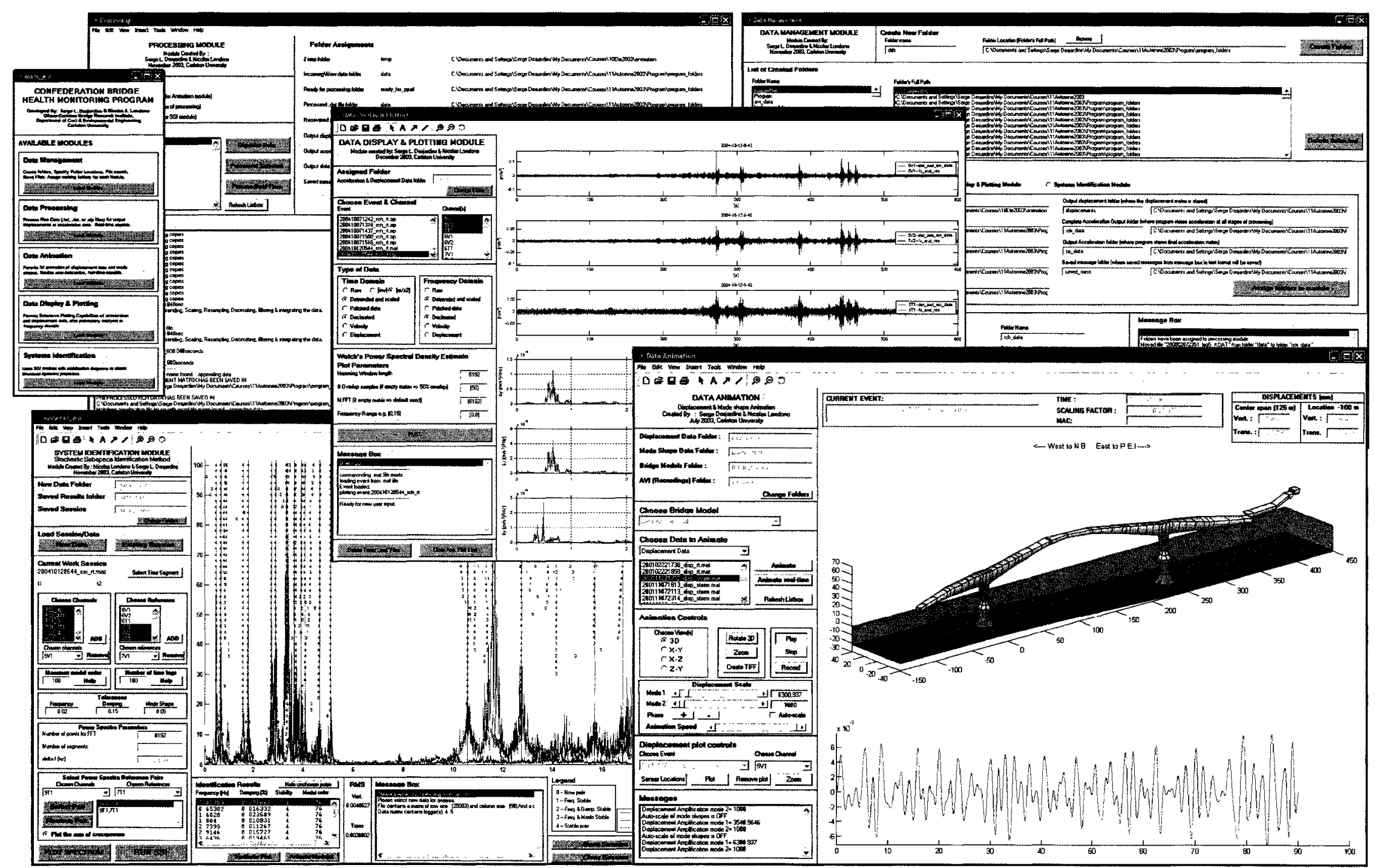

Figure 1.1. Screen-capture of SPLASH modules for Confederation Bridge SHM. 


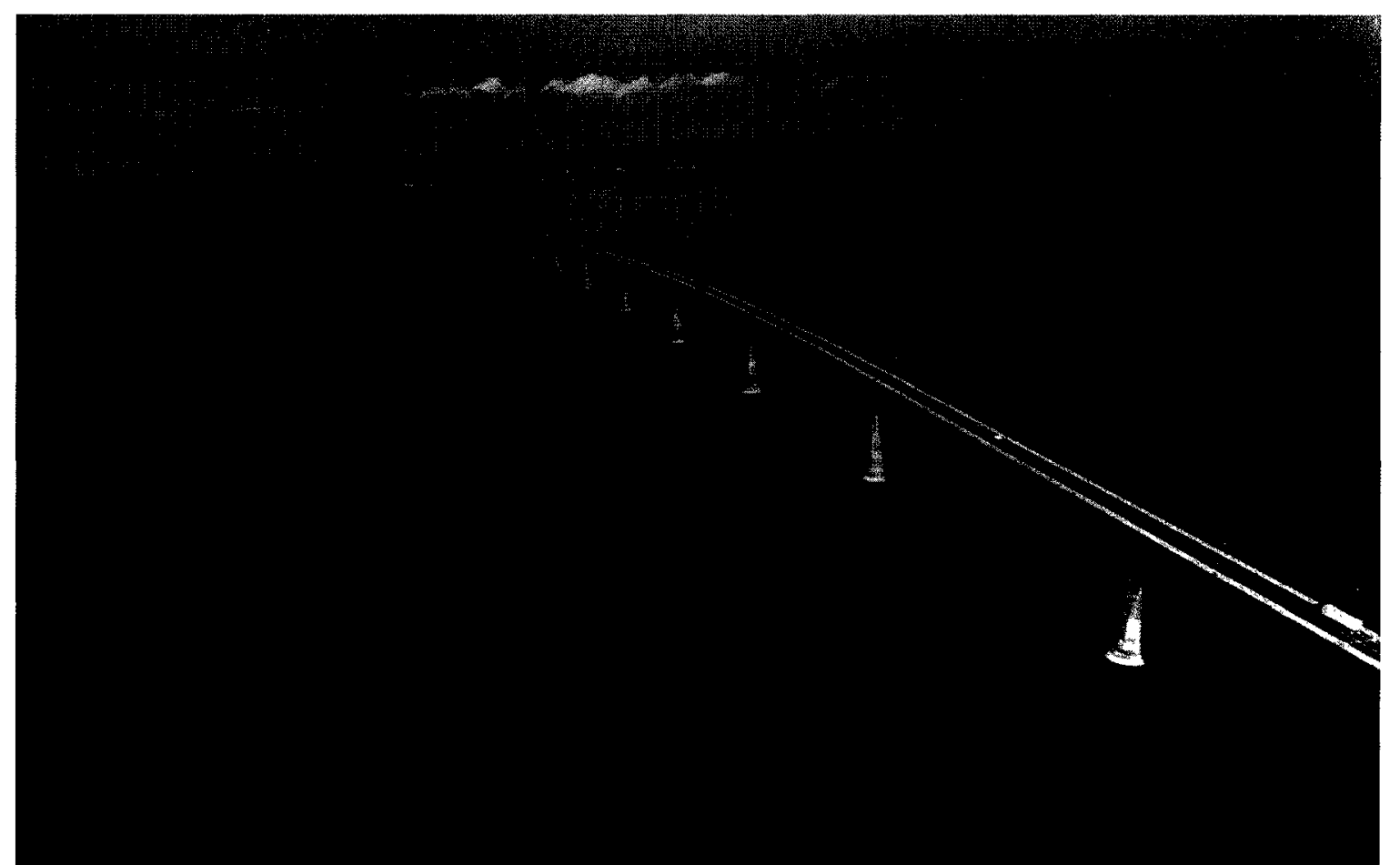

Courtesy of PWGSC

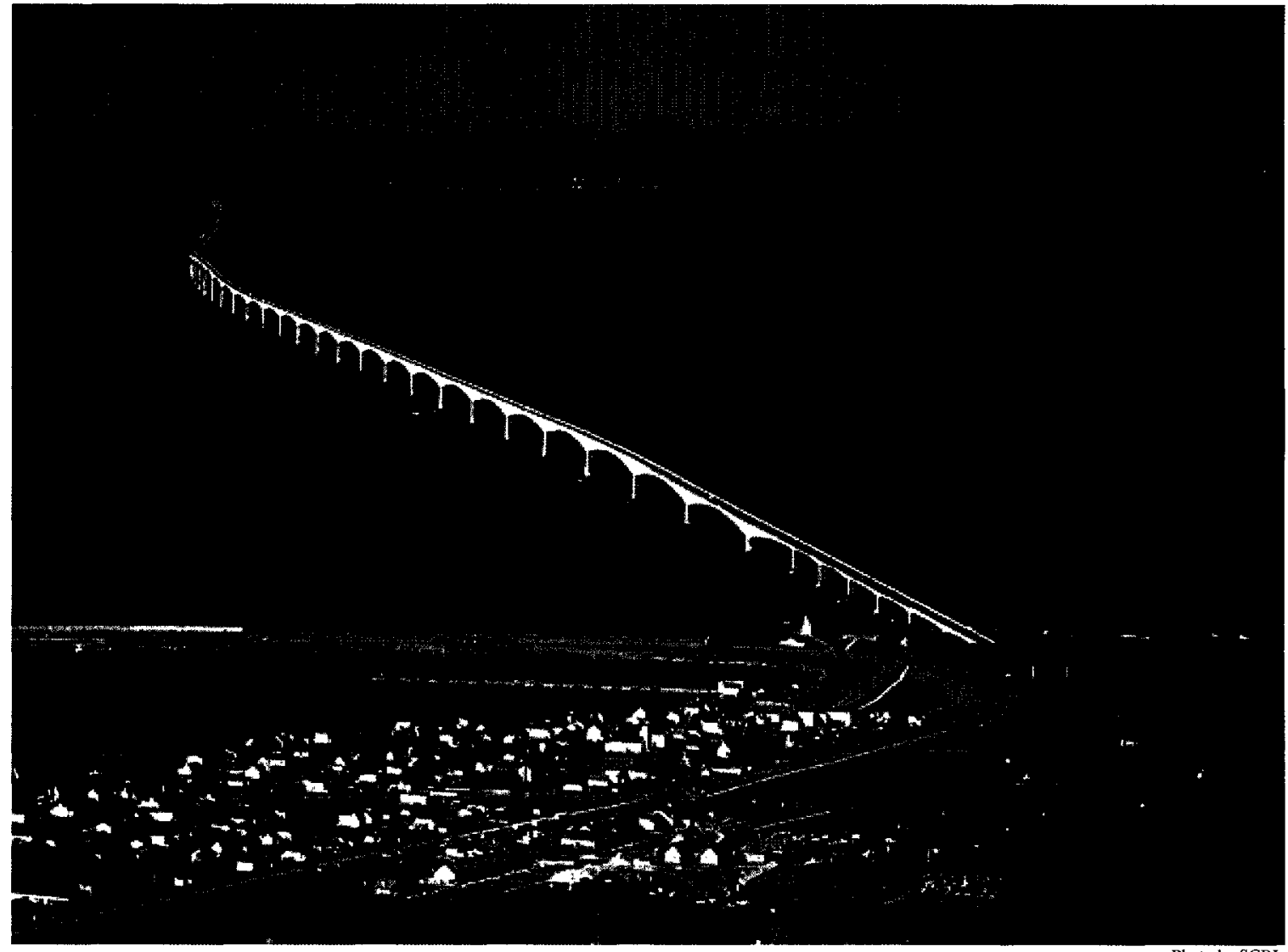

Photo by SCBL

Figure 1.2. The Confederation Bridge. 


\section{Chapter 2}

\section{Vibration-Based Structural Health Monitoring}

Structural health monitoring (SHM) is an emerging new field of interests in civil engineering. It is the study of the wellbeing of new and in-service structures by means of analyzing captured information and data from the structure itself. In recent years, there has been increasing research on non-destructive structural testing and evaluation methodologies in response to the commonly encountered problems of deteriorating infrastructures in many parts of the world. The use of advanced sensing technologies and development of continuous monitoring systems for measuring structural responses on a real-time basis are being studied around the world with promising results. A literature review of SHM and current SHM projects around the world are presented in this chapter. 
The needs of SHM technologies arising from the construction of new structures and maintenance of aging in-service structures are first discussed.

The design and construction of new complex structures are continuously pushing the limits of civil engineering know-how and technologies. Longer, higher and stronger structures relying on new and innovative design concepts and technologies are being constructed all over the world. The designs of these new complex structures are often beyond the scope of current engineering design codes or specifications. Therefore it is of great interest and importance to verify the safety and performance of these modern designs and their adopted design assumptions. A better understanding of the behaviour and performance of these new systems can lead to significant advances in the knowledge base of structural engineering research and practices. Structural health monitoring of innovative and challenging structures can provide critical information and data needed for advancing the state-of-the-art in this new emerging field of research. In this regards for the development of accurate and realistic damage detection methodologies, information and data captured on a new or healthy structure are indispensable benchmarks for later assessment and evaluation of the deteriorated or damaged conditions of the structure as it progresses in its service life. Recent advances have led to rapid developments of new data analysis procedures, data mining and information extraction techniques for analysis of vibration measurement data from continuous monitoring systems. With the rapid developments in advanced computing, some signal processing or data analysis tasks of processing large amounts of data from continuous monitoring systems, which may seem daunting or challenging now can be easily handled a few years from now. 
In Canada, as well as in many other countries in the world, infrastructure renewal is a common major problem, which requires urgent solutions and huge amount of resources to fix. Many of the infrastructures built 40 years ago are now nearing the end of their service life. With limited resources, it is impossible to replace all the old structures. In order to reduce cost, the service life of deteriorated structures can be extended by means of new and innovative technologies, of which SHM is an integral part of this strategy. Thus, informations on the strength and reserved capacities of old structures are often required for rehabilitation or renewal purposes. Current practices of structural evaluations are very much based on site inspection results, which are labor intensive, and can only provide limited information that can accurately reflect the condition of the structure. Period maintenance inspections can only provide stepwise snap-shots of the structural condition, which do not give any detail on the rate of change of the condition and information which may reveal the cause of deterioration.

The need then arises for a new non-destructive evaluation approach which can help to optimize operations of the structures. An evaluation method capable of providing detailed information on the condition of a structure in a continuous real-time basis is an invaluable tool to structural engineers, operators and end-users of the facility. The ability to detect deterioration at an early stage before significant damage, so that early remedial measures can be carried out as part of routine maintenance of the facility, can significantly reduce the total life-cycle cost of the facility while at the same time deliver a higher level of performance. Remote vibration-based SHM systems capture responses of the monitored structure through a sensing medium, which usually comprises of hardware (sensors, data loggers, etc.) connected to a communication network. The captured 
dynamic behaviour and performance of the instrumented structure are continuously monitored over time. Any changes in the structural conditions as reflected by changes or shifts in the dynamic properties, such as frequencies, mode-shapes and damping ratios obtained by applying system identification (SI) techniques, are indications of damage or deterioration in the structure. Research carried out by automotive and aerospace applications shows that an observed change in vibration frequencies over time of a physical system indicates a change in the stiffness of the system which in turn may signify deterioration or damage.

Research in VBSHM is concentrated in the following fields: sensing technologies, data capture hardware, high-speed communication network, signal processing, data-analysis with system identification, condition assessment and finally engineering interpretation.

\subsection{Sensing Technologies}

Extensive research has been carried out to develop new sensing instruments. The sensors are a very important part of any monitoring system. It is where the measurement data are quantified. All information captured by a structural health monitoring system pass through a sensing medium. Sensors are used to quantify the environmental conditions corresponding to the observed structural response measurements, such as wind speed, wind direction, air temperature, solar energy, rainfall quantities, water current speed, ice floe thickness, ice floe speed, earthquake accelerations, and other sources of load or excitations. To quantify structural responses, sensors are used to measure displacements, velocities, accelerations, strains, thermal temperature, material strength, corrosion such as 
that in reinforcing steel, applied forces, etc. As the first link in the complicated chain of a vibration-based SHM system, the quality of the information extracted from the monitoring measurements is dependent on the accuracy of the sensors used to capture the data.

Dynamic responses of a structure are typically measured by accelerometers. Accelerometers can be described as a combination of two transducers. The primary transducer, typically a single-degree-of-freedom vibrating mass converts the acceleration input excitation into a displacement. Then a secondary transducer converts the displacement of the vibrating mass into an electric signal. In most accelerometers the primary transducer is a spring-retained vibrating mass, where accelerations of the structure forces the vibration mass, which is damped and restrained by a spring, to move relative to the casing of the instrument along a single axis. The secondary transducer then responds to the displacement and/or force associated with the vibrating mass. The displacement of the mass and the extension of the spring are proportional to the acceleration only when the oscillation is below the natural frequency of the sensor system. Other accelerometers use a double-cantilever beam as a primary transducer which is very similar to the spring-retained vibrating mass since the dual-cantilever beam can also be modeled as a spring-mass-dashpot system.

Types of secondary transducers, which output an electric signal related to the mechanical displacement, include piezoelectric, potentiometric, reluctive, servo, strain gauge, capacitive, and vibrating element. Presented in Appendix A of this thesis is a summary of workings for each type of accelerometer from Eibeck et al. (2000). 
To summarize, sensing research and knowledge is the basis for a robust VBSHM system. The most widely used vibration sensing instrument is the accelerometer. Important characteristics of accelerometers include range of acceleration level, frequency response, transverse sensitivity (i.e. sensitivity to motion in the non-active direction), mounting errors, temperature and acoustic noise sensitivity, and mass. All these factors need to be well understood and properly adjusted for accurate operation and assured quality of captured information.

\subsection{Data Acquisition Hardware}

The captured signal from the sensors on a VBSHM system must be initially conditioned, filtered and logged by hardware usually installed in the structure or near the sensors. For vibration measurements, a high speed data acquisition system, which incorporates the above mentioned hardware, is needed. As discussed in previous section, the sensors, or accelerometers in the case of vibration measurements, usually transmit the vibration response by electric signal which needs to be conditioned by signal conditioners and filtered by hardware filters to remove frequency contamination from power supplies or other sources. Data-loggers act as the interface for the acquisition of data. A modern data logger typically consists of processors, active memory and high speed communication cards. Data-loggers control the acquisition of data, convert the analog signals into digital readings by high speed sampling, and store the digital information in memory devices. 
An important function of a data acquisition system is the conversion of analog measurement signals from sensors to digital data information. To accurately reflect the structural behaviour of a system and thus to prevent aliasing of the response measurement signals, the vibration measurement information must be sampled at a sampling rate sufficiently high to ensure accuracy and quality of the data by the data-logger. The concept for aliasing can be illustrated by a pendulum analogy. The motion of a pendulum oscillating at $f \mathrm{~Hz}$ is to be captured by taking photographs, as shown in Figure 2.1. If a photograph is taken at a period of over half of the actual period of the oscillation (every two seconds in example shown in Figure 2.1) starting when the pendulum position is at the minimum, it is impossible to correctly reconstruct the motion of the pendulum from these sampled photographs of the system. It is impossible to distinguish whether the pendulum vibrates at the frequency $f(0.375 \mathrm{~Hz}$ in Figure 2.1$)$ or whether it actually vibrates at lower frequency of $f / 3(0.125 \mathrm{~Hz})$. To properly capture the oscillation behaviour of the pendulum vibrating at the frequency of $f \mathrm{~Hz}$, so as to prevent the aliasing error discussed here, it is necessary to sample or take measurement of the vibration response at a sampling frequency at least twice the vibration frequency of the system $\left(F_{s}\right.$ $=2 \times f)$, where $F_{s}$ is the sampling frequency. Sampling at 2 second intervals shown by the dotted red vertical lines in Figure 2.1, which is an inadequately low sampling frequency, results in aliasing errors where the period of the recreated response shown by the red dotted waveform is significantly different from the period of the actual oscillation. Sampling at the time intervals of the black lines of Figure 2.1 at the sampling rate greater than $2 \times f$, where $f$ is the vibration frequency of the oscillating system, creates a reconstructed vibration behaviour, shown in gray in Figure 2.1, more closely representing 
the true oscillation behaviour. The frequency representing half of the sampling frequency $\left(F_{s} / 2\right)$ is called the Nyquist frequency and represents a higher limit for proper capture of an oscillation. To have a good resolution in the representation of the oscillation behaviour, a minimum of 6 to 8 sampling points is typically needed within one cycle of oscillation to properly represent the waveform, as shown in Figure 2.1. Data sampling rate for capturing vibration response of civil structures is typically set between $100 \mathrm{~Hz}$ to $250 \mathrm{~Hz}$ to give good frequency resolution in the frequency range of interest which may vary between 0 to $20 \mathrm{~Hz}$ (Londoño and Lau 2003a). Two other important considerations in the data collection process of a monitoring system are the resolution and range of measurement. These two factors control the accuracy of the measurement and can affect the usefulness of ambient vibration data as a means for detection of damage in a structure. The range of measurement of a data-logger can usually be programmed manually either on-site or remotely depending on the type of monitoring system. The resolution on the other hand is a constant characteristics parameter depending on the sensor or data-logger used to capture and digitize the response measurements. Detailed knowledge of the expected response of the structure is needed prior to setting the range values. If the capture of a structural response to a major earthquake is of importance, the range needs to be appropriately set to accommodate the maximum amplitude of the response. On the other hand, if the ambient vibration response of the structure is of importance, a lower range limit can be set to improve the smoothness of the measurement data. Figure 2.2a shows a response properly captured with adequate range and resolution while Figure $2.2 \mathrm{~b}$ shows the same response improperly captured with either an inadequate range or low resolution. 
With advances in electronics and telecommunication technologies, data acquisition systems are continually improved by faster processors which will enhance the resolution of the system, larger memory to permit storage of larger data tables, higher-speed communication technologies which will allow direct streaming of vibration data for continuous monitoring and remote operation, adaptive filters to improve the quality of the signal by reducing noise contaminations. Research in this field is on-going and will have an impact on the success of VBSHM applications.

\subsection{High-Speed Communication Network}

Another integral component of a successful vibration monitoring project is a good communications network. Data acquisition systems and control computers of a vibration response monitoring system need to be accessible through a robust high-speed local area network. High-bandwidth capacity is vital for transmission of the large amount of data typically associated with vibration monitoring. Remote access to data-loggers for efficient control of their operations and download of the stored monitoring data is also of great importance, so a good internet connection is necessary. For a continuous monitoring project, the data transmission capacity of the network connection for the monitoring system should be greater than the rate of data accumulation. Because of recent advances in telecommunication, optical networks of high bandwidth capacity are continuously being improved and implemented at relatively lower cost. 


\subsection{Data Processing, Analysis \& Condition Assessment}

Significant research needs of VBSHM are in the processing and analysis of the field vibration data for proper structural condition assessment. After the vibration responses are captured by the sensing medium and the data are conditioned, filtered and sampled by on-site data acquisition systems, the resulting conditioned data are then transmitted through communication networks to a central location which may be off-site, for data archival and further more detailed processing and analysis of the data. Monitoring data are often subjected to noise contaminations and the influences of other factors such as the response characteristics and sensitivities of the sensors, resulting in signal drifting or offsets in the data, etc. Therefore numerical data processing procedures are generally needed to enhance the quality of the monitoring data before detailed data analysis can be carried out to extract engineering information from the data. Data analysis may be carried out in the form of spectrum analysis through Fast Fourier Transform (FFT) and/or system identification (SI) using sophisticated numerical processes. As mentioned earlier, many condition assessment algorithms rely on the use of identified modal properties, such as frequencies, mode-shapes and damping ratios, therefore accurate extraction of these information from the monitoring data is an integral part of a structural health monitoring system. Discussions of some SI techniques used in international monitoring projects are presented in Section 2.6. The use of SHM data for damage-identification can be classified into four levels of damage (Doebling et al. 1996). Level 1 damage detection determines if the structure has damage, usually through analysis of time histories of the identified modal frequencies. Level 2 damage detection algorithms identify the geometric location of damage by means of identified frequencies and mode-shapes. 
Level 3 detection quantifies damage severity, and a Level 4 algorithm estimates the remaining service life of the structure. Research on system identification techniques and condition assessment algorithms can be divided in two categories: laboratory testing and full scale testing under actual operating conditions. In a laboratory setting, relatively widespread research has shown successful Level 3 and Level 4 damage identification. However, monitoring systems implemented in actual structures operating in the field have yet to successfully identify presence of damage (Level 1). Much research is therefore needed to deal with the high uncertainty of a full-scale structure excited by ambient conditions. This thesis concerns the processing and analysis of data of a large scale vibration-based monitoring project - the vibration monitoring of the Confederation Bridge.

\subsection{Engineering Reporting/Interpretation}

An important practical component of a VBSHM project is the interpretation and use of information for decision support to engineering practices. Practical use of information extracted from VBSHM data means timely processing and analysis of the monitoring data, and presentation of the results in easy to understand forms, such as real-time visualization and graphical display of response behaviour. Other formats of data analysis summary which show the state or health of the monitored structure may be periodic data $\log$ files that summarize the performance of structure, and alarm systems which signal problems requiring urgent attentions by engineers or operators. Research in this field is mostly based on automation of the monitoring system to quickly and easily provide 
useful information about the state of the structure for decision support to facilitate safe and efficient operation of the monitoring facilities.

\subsection{International Vibration-Based Field Monitoring Projects}

Presented here are five international research projects using field obtained vibration measurements for structural health monitoring, which demonstrate the state of research in the field of VBSHM.

\subsubsection{Hakucho Bridge, Japan}

Hakucho Bridge, completed in 1998, is a 720 m span steel box girder suspension bridge located in a windy and seismically active area of northern Japan at the entrance of Muroran Gulf in Hokkaido Prefecture. This bridge is instrumented with accelerometers at 19 measurement points to capture the vertical motion of the girder due to various types of loading scenario. A study has been carried out to understand the actual dynamic behaviour and loading conditions of the bridge and also to determine the feasibility of using ambient vibration measurements for structural health monitoring purposes (Abe $e t$ al. 2000). This study also presents a data processing and identification scheme for ambient vibration-based monitoring. To process the captured raw data, researchers use the random decrement method to cancel random components and obtain a free vibration time history response (Cole 1973). A time domain system identification method by Ibraham (1977) is employed to identify the dynamic properties of the structure from the 
free vibration responses obtained from the random decrement processing. Natural frequencies, mode-shapes and damping ratios are then correlated with wind and temperature measurements captured at bridge site to study the variability of the obtained dynamic properties. Interesting results are obtained that contradict the expected behaviour from their wind-tunnel experiments conducted prior to construction. Field obtained natural frequencies tend to decrease as wind speed increases. They conclude their study by stating that the processing and identification scheme is applicable to identify modal properties from ambient vibration with high accuracy and has potential to be applied to health monitoring of civil structures.

\subsubsection{Vasco de Gama Bridge, Portugal}

The Vasco de Gama Bridge, crossing the Tagus River in Lisbon, Portugal, is about $12 \mathrm{~km}$ long and includes a $420 \mathrm{~m}$ cable stayed span over the navigational channel. A study has been carried out using ambient vibration measurements to assess the aerodynamic and seismic behaviour of the bridge and to compare two identification schemes (Peeters et al. 2002). An ambient vibration test was performed on the bridge deck which is $31 \mathrm{~m}$ wide and is formed by two lateral pre-stressed concrete girders connected by slab and transversal I-beams. The ambient vibration test was carried out using 2 reference 16 bit GSR-16 tri-axial strong motion recorders permanently installed on the bridge. Two other 'scanning' tri-axial motion recorders were set-up at 25 different locations along the bridge deck. Measurement records were taken for a length of 16 minutes for each set-up. Ambient response was excited by wind which varied from $1 \mathrm{~m} / \mathrm{s}$ to $22 \mathrm{~m} / \mathrm{s}$ during the test. The two system identification methods covered in this study were the simple peak 
picking method and the more advanced stochastic subspace identification method. The peak picking method consists of identifying eigenfrequencies as the peaks of power spectrum plots and assumes low damping and well separated frequencies of the system. The simplicity of this method inevitably results in some difficulties and inaccuracy of the results. Peak identification is a very subjective task; if spectrum peaks are not clearly identifiable, as is often the case of field monitoring data, errors in modes may thus result. It is important to note that the peak picking method does not identify mode-shapes of the response but rather the operational deflection shapes which may contain a combination of mode-shapes of closely spaced modes and that the damping estimates are not included in the identified properties. The stochastic subspace identification (SSI) method uses a stochastic state space model which is identified directly from the measured output data. Studies have shown that a stochastic state space model is a good representation of a vibrating structure excited by unknown forces, which are assumed to be white noise signals (Peeters 2000, Peeters \& DeRoeck 1999). The SSI method compared well with the peak picking method in this study albeit with a few advantageous differences, the SSI method clearly identifies more low frequency modes in the range of $0-1 \mathrm{~Hz}$ and also identifies damping ratios along with the mode-shapes and eigenfrequencies of a vibrating structure. The stochastic subspace identification method is presented in detail in Chapter 5 of this thesis. Peeters et al. (2002) conclude from their study on the vibration data of the Vasco de Gama Bridge that the SSI method is a more complete identification method. It is also stated that the need for free vibration tests becomes less significant since the SSI method can accurately determine damping ratios along with the frequencies and modeshapes from ambient measurement responses of vibrating structures. 


\subsubsection{Oresund Bridge, Denmark/Sweden}

The Oresund Bridge is part of the fixed link between Copenhagen (Denmark) and Malmo (Sweden). The cable stayed bridge has a main span of $490 \mathrm{~m}$ and two side spans of $160 \mathrm{~m}$ and $141 \mathrm{~m}$. The bridge deck has two levels, the lower level has two railway tracks and the top level has a four lane motorway. The bridge deck is connected to the two H-shaped pylons, the top of which are $204 \mathrm{~m}$ above sea level, by 10 pairs of cables on each side. A permanent PC-based continuous monitoring system has been installed on the cable stayed portion of the fixed link. This monitoring system measures both static and dynamic load and response parameters such as temperature, wind characteristics, air humidity, strains and accelerations. A study into the dynamic behaviour of the bridge was carried out by Peeters et al. (2003). A detailed presentation of the dynamic monitoring system installed by GeoSIG (called CR-4 Central Recorder) including sensor information and data transmission is included in the reference. Advanced software is essential in this type of high speed monitoring system. While other studies fail to mention what type of software was employed, either commercial or custom, this study on the Oresund Bridge includes detailed information on a windows-based data acquisition and processing software for the CR-4 system. Three different application tools are utilized in this project; SEISLOG, a data acquisition software, CENTRAL, an interface for the remote access of the CR-4 systems, and CMS (civil monitoring system), a processing tool for the static data acquired by the system. Although operational modal analysis, a dynamic analysis using output only data with no detailed knowledge of input excitation, was carried out, no software for modal analysis is mentioned in this study. A detailed dynamic analysis was carried out using a 5-minute recording of the 85 channels. The identification technique 
used in this study is the stochastic subspace identification method discussed earlier and in more details in Chapter 5. Identification results from responses measured by sensors placed directly on cables were successfully used to calculate the cable tensions from the obtained eigenfrequencies. Deck and tower vibrations were also analyzed using the SSI method and some low frequency modes were accurately identified. Due to the fact that most sensors were placed on stay cables, limited information on mode-shapes of the deck and towers were obtained. The study concludes by stating that operational modal analysis applied to dynamic data can provide useful information on the health of the bridge and its components.

\subsubsection{Runyang Yangtze River Bridge, China}

The Runyang Yangtze River Bridge in China is composed of a cable-stayed bridge and a suspension bridge. The cable-stayed portion is a steel box girder bridge with three spans $(176 m+406 m+176 m)$, double pylon towers and double cable faces. The suspension bridge portion is a single simply supported steel box girder bridge with a main span of $1490 \mathrm{~m}$. A report has been published on the health monitoring system installed on this super bridge ( $\mathrm{Li}$ et al. 2003). The sensor system includes anemoscope, temperature transducer, displacement meter, GPS, strain gauge, force transducer, axle speedometer, acceleration transducer, signal amplifier and regulator. The data acquisition system includes several micro-computer-controlled data acquisition stations including stations installed inside the bridge structure. Each station collects data from the sensors, regulates the signal readings, preliminarily processes and stores the collected data, and then transmits them to the data processing and analyzing system in the monitor center via the 
high-speed optical fiber network. The monitoring management center has many high performance workstations for the various tasks of data processing, graphic display of structural information, structural analysis, system control and management, maintenance and diagnosis. The report by $\mathrm{Li}$ et al. (2003) presents the monitoring system and its physical components and gives a detailed review of VBSHM. It does not however present any findings or research results from the acquired data of the system. They state in their conclusion the current difficulty of using ambient vibration measurements and their extracted modal parameters to detect damage since uncertain factors in the structure and the complicated working environment have an adverse effect on the modal sensitivity. More research is then needed to fully understand the behaviour of the data.

\subsubsection{Akashi-Kaikyo Bridge, Japan}

The Akashi-Kaikyo Bridge spans the Akashi Strait between Maiko, Tarumi-ward in Kobe, and Matsuho on Awaji Island. Its main span of $1991 \mathrm{~m}$ makes it the longest suspension bridge in the world. A full-scale measurement of vibration study was carried out by Katsuchi et al. (2003). The vibration-based monitoring system was installed on this bridge for the purposes of design verification and monitoring of structural condition during environmental events such as typhoons and earthquakes. The main focus of the study was to identify modal parameters particularly modal damping from ambient vibration data. The vibration data was collected by GPS and velocity sensors during relatively calm days to avoid aerodynamic effects. The identification technique in this study employed the random decrement method (Cole 1973). The identified modal frequencies are 2 to $10 \%$ higher than analytical results which they say agrees with other 
studies. Damping ratios are also higher than the analytical values but by a higher margin. They conclude by stating that more research and analysis is needed to obtain more accurate results and to clarify vibration characteristics. One way to improve results is not to use the displacement data obtained from the GPS instruments for vibration analysis since these sensors have a relatively low resolution of about $1 \mathrm{~cm}$, which is high for GPS standard but not very useful for measurement of ambient vibrations. The GPS sensors also have a very low sampling rate of $1 \mathrm{~Hz}$. These two factors can significantly affect the accuracy of the modal identification results from the field-obtained monitoring data.

\subsubsection{Discussion}

The recent studies on VBSHM give a general idea on the direction of this emerging new field of research. They show that vibration measurement offers a viable means to identify structural dynamic properties. The projects discussed have used measured data to obtain the modal properties via different identification schemes. All results seem to correlate well with the analytical properties to differing degrees. Different sensing instruments have also been shown to give acceptable results, some more accurate than others. Obtaining modal properties from response only monitoring data is relatively simple and straight forward to achieve, but to achieve it in a continuous manner with the accuracy and robustness required for realistic condition assessment of the system is extremely challenging and has not, as of yet, been realized. Some studies have performed many identification analyses and found variability in the modal parameters. The next step is to fully understand the behaviour of the identification results by trying to correlate the variability observed in the modal parameters with environmental effects or 
other potential causes. In order to carry out such research, significant obstacles must be overcome in the way of rapid and efficient data processing, analysis and visualization techniques. This demonstrates the need of a centralized computer platform for various data processing, data analysis and interpretation aspects of vibration-based SHM applications. 


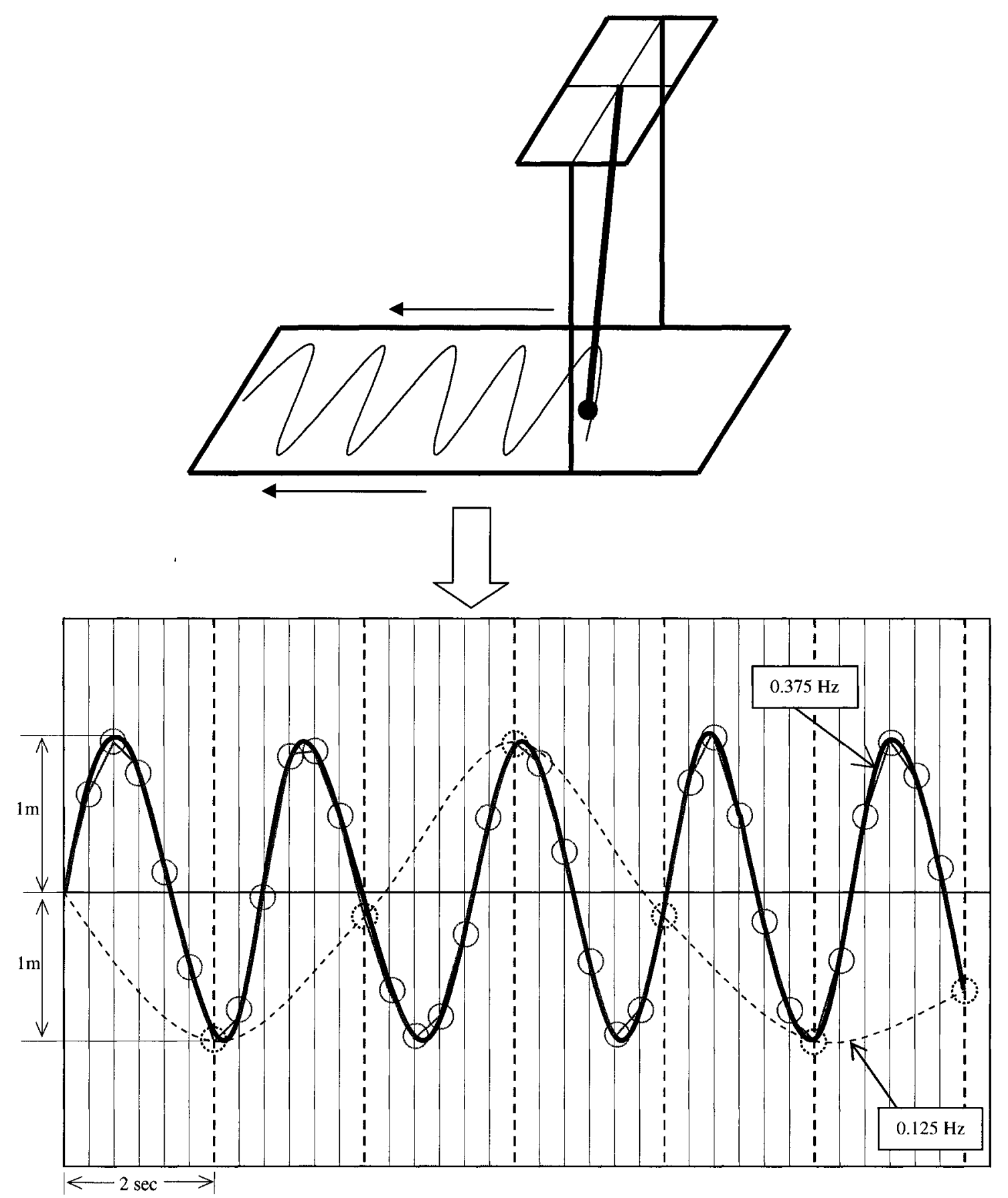

Figure 2.1. Pendulum analogy for aliasing problem in sampling. 

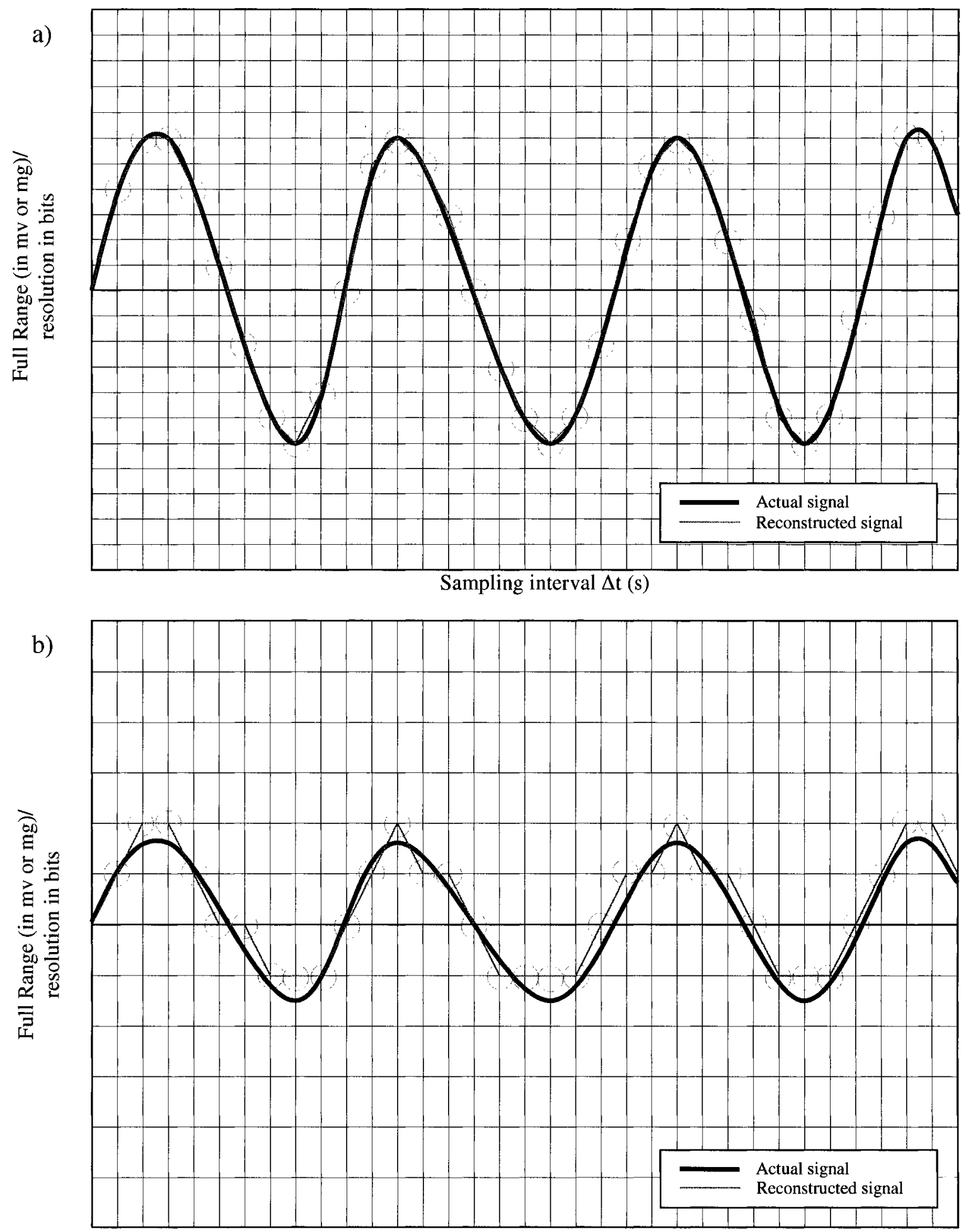

Sampling interval $\Delta \mathrm{t}(\mathrm{s})$

Figure 2.2. Resolution and range of data-logger sampling. a) Good range and resolution b) High range and/or low resolution 


\section{Chapter 3}

\section{Monitoring of the Confederation Bridge}

The Confederation Bridge at 12.9 kilometers is the world's longest bridge over icecovered sea-water. It was open for traffic on June $1^{\text {st }} 1997$. It spans the Northumberland Strait linking Prince Edward Island with the province of New-Brunswick in eastern Canada, as shown in Figure 3.1. The very stringent design criteria because of severe environmental conditions at the bridge location make the Confederation Bridge a unique structure. The seasonal ice floes and harsh wind conditions in the Northumberland Strait makes the design of the Confederation Bridge very challenging. In this Chapter the design criteria and structural layout of the Confederation Bridge are discussed. 


\subsection{Northumberland Strait}

The Northumberland Strait is a natural sea channel between mainland Canada along the coast of the province of New-Brunswick and Prince Edward Island. The channel is approximately $300 \mathrm{~km}$ long and $55 \mathrm{~km}$ wide at its broadest section. It is approximately 12.9 kilometers wide at the site of the Confederation Bridge. Weather records show that harsh weather conditions often occur at the bridge site. The bridge is subjected to frequent strong winds of gusts up to $100 \mathrm{~km} / \mathrm{h}$. Hurricanes originating from the east coast of the United States and Canada can lead to wind gusts up to $175 \mathrm{~km} / \mathrm{h}$ at bridge site. Seasonal variations in temperatures can range from $-40^{\circ} \mathrm{C}$ in winter to highs around $35^{\circ} \mathrm{C}$ in mid-summer. During fall and spring, daily temperature variations often result in freeze/thaw cycles that can have adverse effects on the durability of any marine structures. Strong tidal currents occur twice daily in the Northumberland Strait which result in strong drag forces on the structure. During winter, these tidal currents drive large ice floes against the bridge piers. The surface ice floes in the Northumberland Strait can reach a depth of $3 \mathrm{~m}$ and impose large lateral loads on the bridge piers. Earthquake loads are also of concern in the design of the Confederation Bridge, particularly because of its long design service life. The harsh environmental conditions in the Strait make the design and construction of the Confederation Bridge a very challenging task. 


\subsection{Design Criteria of the Confederation Bridge}

Due to the importance of this structure and its location in a harsh climate, its design specifications and requirements are more stringent than those of typical highway bridges and are not covered in any bridge design standards and codes. The main performance requirements of the Confederation Bridge are a design service life of 100 years, which is twice that of ordinary structures, and a structural safety level quantified by the reliability index of $\beta=4.0$ for load effects with multiple load paths, and $\beta=4.25$ for load effects of single load path, as compared to the values for ordinary structures of $\beta=3.0$ to $\beta=3.5$, respectively. To satisfy these stringent design specifications under the harsh environmental conditions of the Northumberland Strait, the designers of the Confederation Bridge had to overcome numerous challenges, including lack of data and information on the various design load effects of ice impact, traffic, wind and earthquakes. Load combinations and resistance factors have been specially developed for the project (MacGregor et al. 1997).

\subsection{Structural Layout of the Confederation Bridge}

The Confederation Bridge is a pre-stressed concrete bridge. It crosses the Northumberland Strait in Eastern Canada and provides the only fixed link between New Brunswick in mainland Canada and Prince Edward Island. With a length of $12.9 \mathrm{~km}$, the Confederation Bridge is the world's longest continuous multi-span bridge operating in ice-covered water. To cover the long shore-to-shore distance the bridge is divided into 
21 approach spans, two transition spans of $165 \mathrm{~m}$ each and 43 main spans of $250 \mathrm{~m}$ each at a typical height of $40 \mathrm{~m}$ above the mean sea level. The main-spans portion of the bridge is comprised of 22 repetitive structural frame modules of $500 \mathrm{~m}$ length each. Each frame module is a $440 \mathrm{~m}$ portal frame made up of a $250 \mathrm{~m}$ centre span and two $95 \mathrm{~m}$ overhangs, one on each side of the centre span, plus a $60 \mathrm{~m}$ simply supported drop-in expansion span, as shown in Figure 3.2. The portal frame is constructed of four types of prefabricated component units, pier base, pier shaft, main girder and fixed drop-in span girder. The connections between the different units of the portal frame are made continuous by post tensioning pre-stressing and grouting. The bridge girders are single trapezoidal cell box girders with section depth varying from $14 \mathrm{~m}$ above the piers to 4.5 $\mathrm{m}$ at mid-span of the drop-in girder, and a cross-section width varying from $5.0 \mathrm{~m}$ at the bottom to $7.0 \mathrm{~m}$ at the top, with $2.5 \mathrm{~m}$ deck overhangs.

\subsection{Confederation Bridge Monitoring Project}

A comprehensive long-term monitoring system on the Confederation Bridge has been in operation since the bridge opening in 1997 to collect data and information about its behaviour and performance. The monitoring system measures and records both environmental and bridge response data related to wind, ice forces, short and long-term deflections, thermal effects, traffic loading, corrosion and dynamic responses. All instruments incorporated in the bridge structure are located in two adjacent spans, a rigid frame and a drop-in simply supported span covering a one kilometer stretch of the bridge 
in the deep-sea part of the Northumberland Strait with some additional wind measurements being also taken at the highest elevation of the navigation span.

\subsubsection{Environmental and Materials Monitoring}

Two anemometer units are placed at the navigation span and two more are located at the instrumented span. The critical wind data, which includes wind speeds and direction, are measured at each anemometer location. All three components of wind speed are measured (transverse, longitudinal and vertical). The long-term data can be utilized to compare with the test data obtained by the wind tunnel tests by King and Davenport (1997) to correlate modal properties between the theoretical laboratory results and the actual data from the field determined from the vibration monitoring project.

In the monitoring project, instrumentations are provided to obtain data to verify the ice force design assumptions of the bridge. These instruments are incorporated in adjacent piers. The ice force monitoring can be separated into three components. The first component is indirect determination of the global ice force by measuring the response of two piers with highly sensitive bi-axial tilt meters placed inside each pier at two locations. The second component is the direct measurement of local ice pressure by means of ice load panels installed on one ice shield and pier base. These ice panels are double walled with internal strain-gauged load sensors. The third component is the observation of ice movements and conditions by means of four time lapse video cameras located on bridge deck, two sonar and a laser located on sea bed to measure the keel depth and freeboard of the ice as shown in Figure 3.3. Details of ice force monitoring of the Confederation Bridge have been presented by Cheung et al. (1997). 
For traffic monitoring, vehicle configuration and loads are monitored using recording video cameras and weight stations. Strain gauges are installed along the instrumented span to measure vehicular loads. Dynamic effects of moving vehicles during normal operations can be correlated with controlled vehicular loading test data. The structural responses to traffic load are recorded by the dynamic monitoring system of the bridge.

Strength and modulus of elasticity of concrete vary with time. For material property monitoring, the mechanical properties of 18 cylinders of concrete from three arbitrarily chosen concrete casts during construction of the Confederation Bridge are monitored. The cylinders are tested for values of their strength and modulus of elasticity at 6 selected ages. The material properties from the cylinder tests are compared to design values by various concrete design codes and standards. Details of materials monitoring results from the Confederation Bridge can be found in the reference Ghali et al. (2000).

The creep and shrinkage properties of the concrete cylinders are monitored. Five pairs of concrete cylinders are subjected to the same weather and curing conditions as the bridge elements. They are stored inside the box girder sections. A loading device, as shown in Figure 3.4, is used to determine creep and shrinkage values by subjecting the $100 \mathrm{~mm}$ cylinder to different loadings representing five different stages of construction. The measurement data are compared to a companion cylinder that has not undergone any loading to determine the relevant values of creep and shrinkage (Cheung et al. 1997; Ghali et al. 2000; Ghali 1996).

The objective of the thermal effects part of the monitoring system is to obtain data for improvement of the prediction model capabilities to reliably predict long-term thermal stresses and thermal cracking behaviour of large size concrete structures. The monitoring 
data are also used to compare the actual field parameters with design assumptions. Temperature is recorded by thermocouples installed at seven locations on the instrumented span. In each section, thermocouples are installed at different thickness positions. Vibrating wire gauges are used at these locations to determine strain distribution. To measure the intensity of solar radiation, pyranometers are installed on the top and side surfaces of the girder. Thermocouples are installed to measure ambient temperature inside the box girders.

The Confederation Bridge is located in a very rich chloride and sulphate environment. Corrosion of reinforcing steel is therefore a concern of engineers. Continuous monitoring of the corrosion state of reinforcing steel in the splash zone of a pier is conducted as part of the monitoring program (Cheung et al. 1997).

Compared to other large scale monitoring projects around the world, some of which are discussed in Chapter 2, the Confederation Bridge monitoring project is unique in that its data sets are particularly valuable because of the comprehensive nature of the monitoring systems covering a wide range of aspects important to the behaviour and performance of the structure. Data sets from one monitoring area are available and can be readily utilized in conjunction with another monitoring area to examine correlations in such detail never or seldom feasible before; as in the case of the investigation study of variation and sensitivity of vibration modal behaviour of the bridge to ambient temperature variations of the environment co-authored with Londoño and Lau (Londoño et al. 2004a) and discussed in detail in Chapter 7 of this thesis. 


\subsubsection{Vibration-Based Monitoring}

The dynamic monitoring system is dedicated to the measurement of the ambient vibration responses of the bridge caused by significant sources of dynamic excitations, including wind, heavy traffic, ice loads and earthquakes. The vibration instrumentation comprises 76 accelerometers distributed around a typical structural frame module, as shown in Figure 3.5. The response behaviour observed in the instrumented segment of the bridge is considered representative of the behaviour of the main span portion of the structure. Vibration responses of the bridge girders are measured in the vertical and transverse directions as shown in Figure 3.5. This setup facilitates the recovery of vertical bending, transverse bending and torsional vibration modes of the bridge superstructure. The vibration sensors used in the monitoring system include both piezo-electric accelerometers and servo accelerometers. Locations of the sensors are listed in Table 3.1 and shown in Figure 3.5. The types and specifications of accelerometer sensors used in the monitoring project are listed in Table 3.2. The piezo-electric accelerometers are PCB Model 393C, shown in Appendix B, and the servo accelerometers are Allied Signal QLC 400 and QA 1200.

The measured analog accelerometer signals are conditioned and filtered for anti-aliasing by an 8-pole $50 \mathrm{~Hz}$ low-pass Bessel filter. Signals are then sampled and digitized by a network of distributed high-speed data loggers before being sent to on-site computers and transmitted back to the research team at Carleton University in Ottawa for data archival and research. The data loggers are CR9000 with $16 \mathrm{Bit} 100 \mathrm{kHz}$ A/D conversion from Campbell Scientific, shown in Appendix B. They operate in continuous buffered data collection mode, which upon triggering by detection of specific dynamic events, such as 
heavy traffic signals or high winds, or simply upon user request, store time history response data in data tables in memory. Otherwise, only statistic information determined from the time history data, such as mean, maximum, minimum and standard deviation etc, are stored to establish long-term trends in the behaviour of the bridge. Triggered data, typically sampled at $100 \mathrm{~Hz}$ to $167 \mathrm{~Hz}$, are stored temporarily in logger memory until retrieval at specified scheduled intervals from a remote network computer assigned to the control and operation of each logger. The logger computer retrieves the data from the data logger and sends them to a centralized platform where they are made accessible over the internet to researchers at the Ottawa-Carleton Bridge Research Institute (OCBRI) at Carleton University in Ottawa, approximately 1300 kilometers away, for data processing and analysis. Data acquisition software is installed in each logger control computers and can be remotely accessed for system control or maintenance. This software controls the acquisition of data from the sensors connected to the data-loggers and collection of data from the loggers to the control computers. It also permits editing of logger programming code to modify the functioning of the logger. Figure 3.6 illustrates the steps and processes of high-speed dynamic monitoring data collection. Details of the monitoring system setup have been described in the references (Montreuil et al. 1998, Cheung et al. $1997 \& 2002)$.

With the completion of the Bridge and the experiences of its successful operation since 1997 , there is the opportunity to assess and verify the behaviour and performances of this structure using the bridge response monitoring data collected since its opening and thus advance the knowledge and practices of bridge engineering. For structural behaviour 
verification, vibration-based measurement techniques are employed to obtain actual performance data of the structure in the field. 
Table 3.1. Sensor location with make and model of accelerometers. Location numbers in table correspond to location numbers in Figure 3.5.

\begin{tabular}{|c|c|c|c|c|c|c|c|c|}
\hline \multirow{2}{*}{$\begin{array}{l}\text { Pier } \\
\text { (Data Logger } \\
\text { Number) }\end{array}$} & \multirow{2}{*}{ Location } & \multicolumn{5}{|c|}{$\begin{array}{l}\text { Channel numbering inside } \\
\text { data-loggers }\end{array}$} & \multirow{2}{*}{$\begin{array}{l}\text { Scale } \\
\mathrm{V} / \mathrm{g}\end{array}$} & \multirow{2}{*}{$\begin{array}{l}\text { Accelerometer } \\
\text { Model }\end{array}$} \\
\hline & & $\mathrm{T}_{1}$ & $\mathrm{~T}_{2}$ & $\mathrm{~L}$ & $\mathrm{~V}_{1}$ & $V_{2}$ & & \\
\hline \multirow[t]{5}{*}{ P 30} & 1 & 7 & & 8 & 9 & & 1.00 & PCB 326 A03 \\
\hline & 3 & 12 & & 13 & & & 2.66 & QLC 400 \\
\hline & 4 & 10 & & 11 & & & 2.66 & QLC 400 \\
\hline & 16 & & 3 & & 1 & 2 & 1.00 & PCB 393C \\
\hline & 17 & & 6 & & 4 & 5 & 1.00 & PCB 393C \\
\hline \multirow[t]{11}{*}{ P31 } & 1 & 1 & & 2 & 3 & & 1.00 & PCB 326 A03 \\
\hline & 2 & 4 & & 5 & 6 & & 1.00 & PCB 326 A03 \\
\hline & 3 & 7 & & 8 & & & 99.0 & QA 1200 \\
\hline & 4 & 9 & & 10 & & & 99.0 & QA 1200 \\
\hline & 10 & & 30 & & 29 & 31 & 1.00 & PCB 393C \\
\hline & 11 & & 26 & & 28 & 27 & 2.66 & QLC 400 \\
\hline & 12 & & 15 & & 16 & 14 & 1.00 & PCB 393C \\
\hline & 13 & & 17 & & 19 & 18 & 2.66 & QLC 400 \\
\hline & 14 & & 20 & & 21 & 22 & 1.00 & PCB 393C \\
\hline & 15 & & 23 & & 25 & 24 & 2.66 & QLC 400 \\
\hline & 18 & 11 & & 12 & 13 & & 99.0 & QA 1200 \\
\hline \multirow[t]{9}{*}{ P.32 } & 1 & 1 & & 2 & 3 & & 1.00 & PCB 326 A03 \\
\hline & 2 & 4 & & 5 & 6 & & 1.00 & PCB 326 A03 \\
\hline & 3 & 10 & & 11 & & & 99.0 & QA 1200 \\
\hline & 4 & 16 & & 15 & & & 99.0 & QA 1200 \\
\hline & 5 & 24 & & & 23 & 25 & 1.00 & PCB 393C \\
\hline & 6 & 28 & & & 26 & 27 & 1.00 & PCB 393C \\
\hline & 7 & 30 & & & 29 & 31 & 1.00 & PCB 393C \\
\hline & 8 & 32 & & & 34 & 33 & 1.00 & PCB 393C \\
\hline & 9 & 20 & & & 21 & 22 & 2.66 & QLC 400 \\
\hline \multirow{3}{*}{$\begin{array}{r}\text { P33 } \\
\text { (No.6) }\end{array}$} & 1 & 1 & & 2 & 3 & & 1.00 & PCB 326 A03 \\
\hline & 3 & 4 & & 5 & & & 2.66 & QLC 400 \\
\hline & 4 & 6 & & 7 & & & 2.66 & QLC 400 \\
\hline
\end{tabular}


Table 3.2. Specifications for accelerometers located on Confederation Bridge.

\begin{tabular}{|c|c|c|c|}
\cline { 2 - 4 } \multicolumn{1}{c|}{} & PCB 393C (piezo-electric) & $\begin{array}{c}\text { QLC 400 } \\
\text { (servo) }\end{array}$ & QA 1200 (servo) \\
\hline $\begin{array}{c}\text { Principal location } \\
\text { Sensitivity }\end{array}$ & Along girder & Along girder & $\begin{array}{c}\text { In piers } \\
\text { (Initially for Ice } \\
\text { floe monitoring) }\end{array}$ \\
\hline Frequency range & $0.01 \mathrm{~Hz}-1200 \mathrm{~Hz}( \pm 10 \%)$ & $0-300 \mathrm{~Hz}( \pm 10 \%)$ & $0-300 \mathrm{~Hz}( \pm 10 \%)$ \\
\hline Amplitude range & $\pm 1.5 \mathrm{~g}$ & $\pm 10 \mathrm{~g}$ & $\pm 25 \mathrm{~g}$ \\
\hline $\begin{array}{c}\text { Resolution } \\
\begin{array}{c}\text { Operational } \\
\text { temperature } \\
\text { range }\end{array}\end{array}$ & $0.1 \mathrm{mg}$ & $0.01 \mathrm{mg}$ & $0.005 \mathrm{mg}$ \\
\hline
\end{tabular}




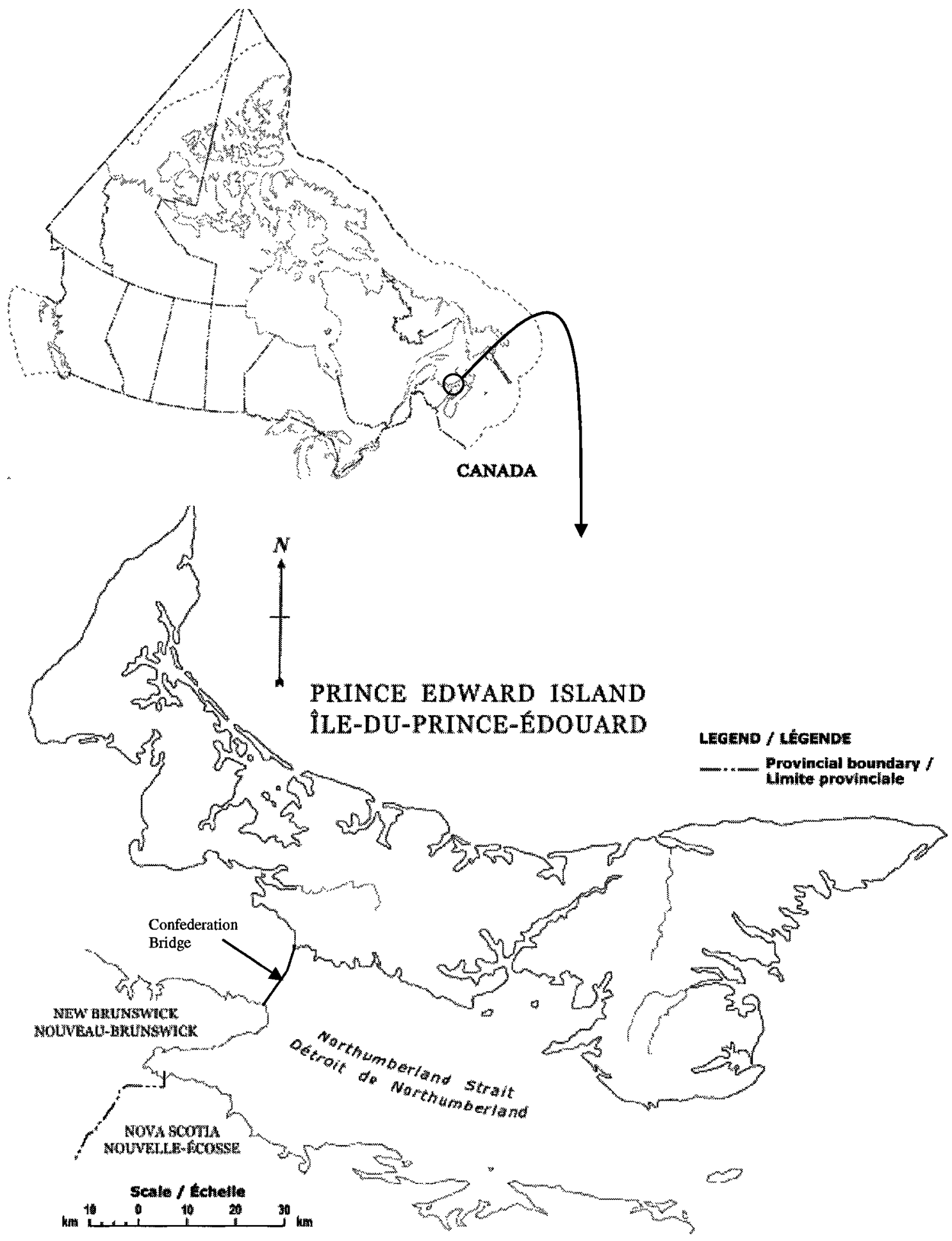

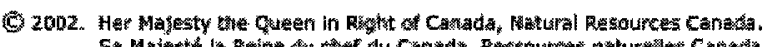

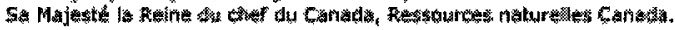

Figure 3.1. Location of Confederation Bridge. 


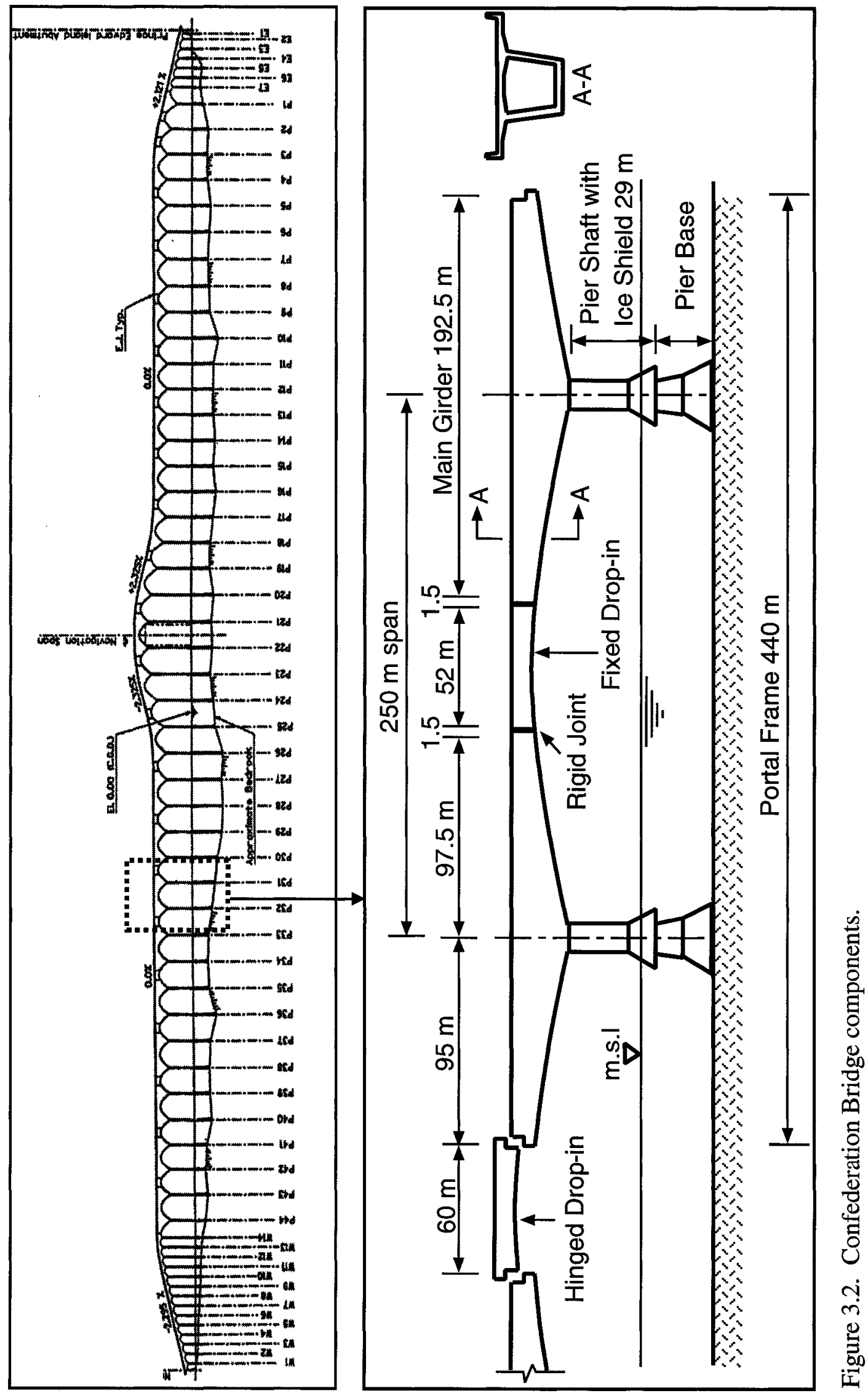



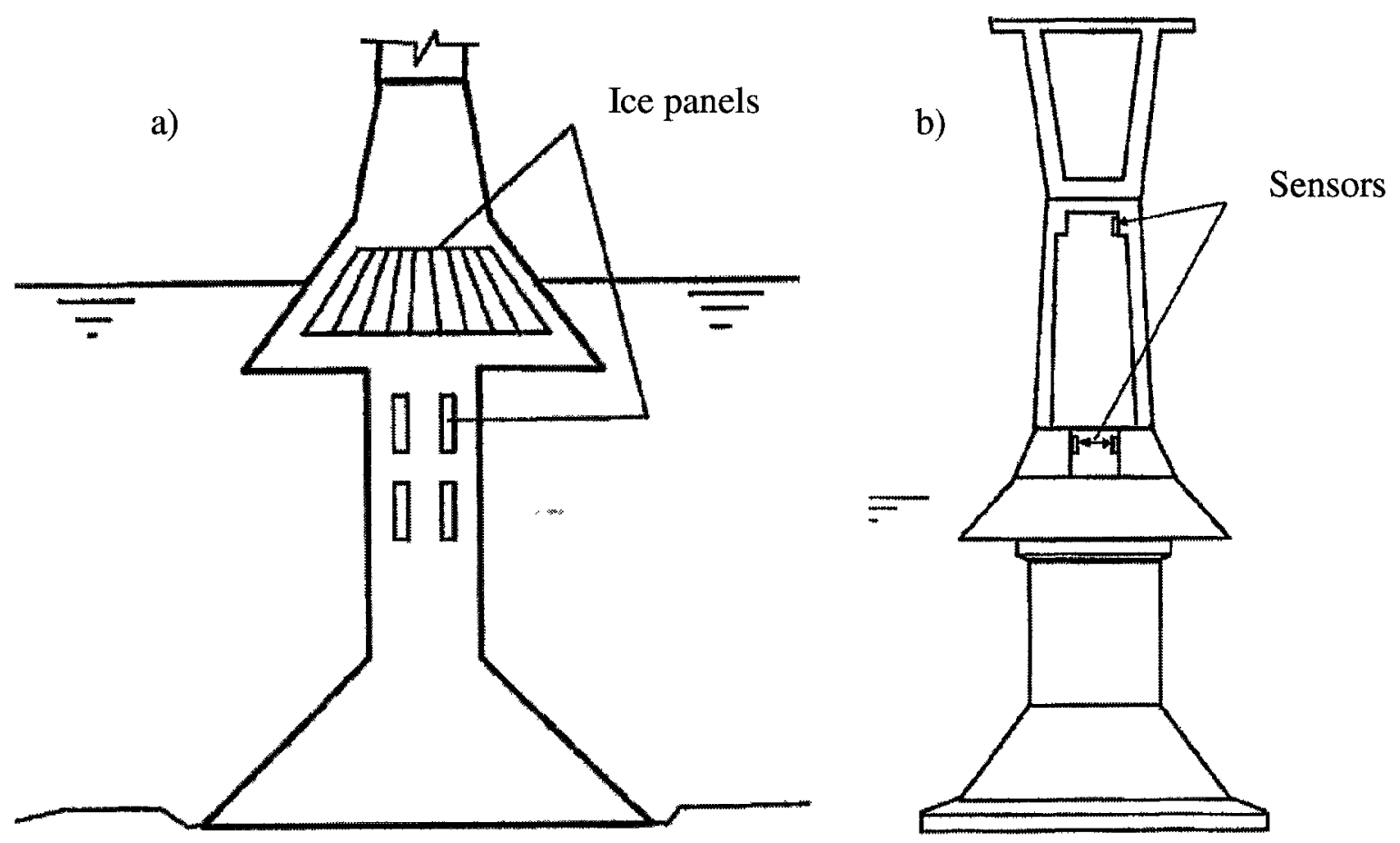

c)

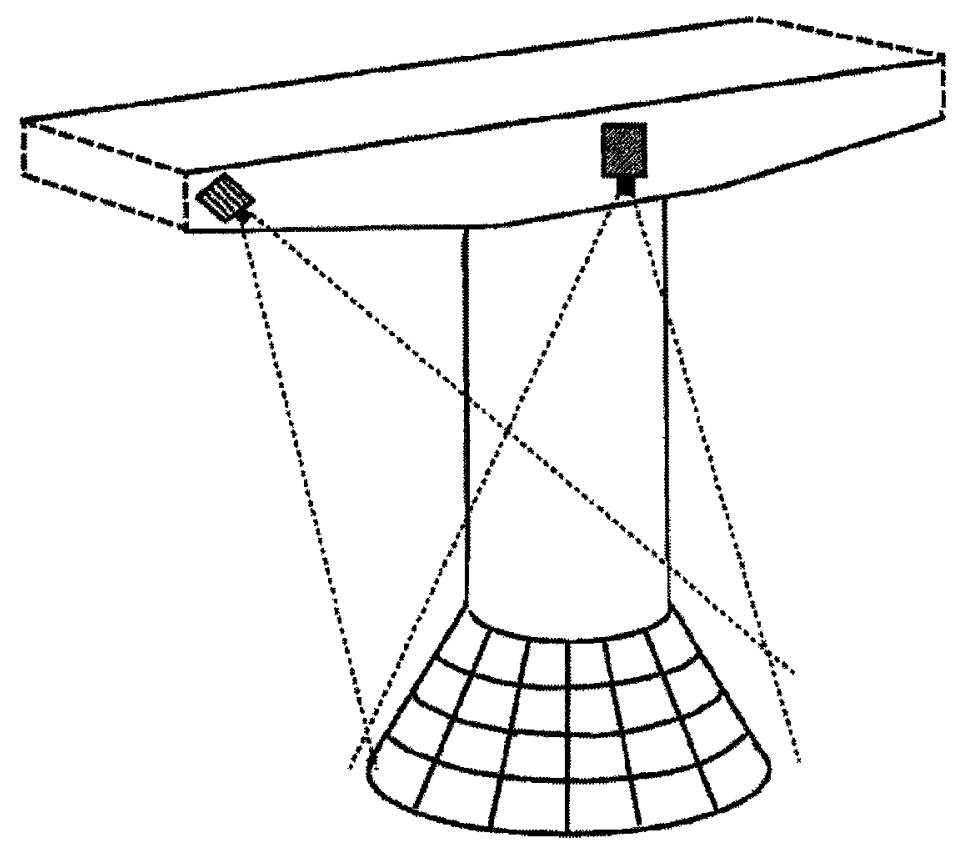

Figure 3.3. Ice monitoring instrumentation of the Confederation Bridge. a) Ice panels; b) Tilt meters; c) Video cameras. (Cheung et al. 1997) 


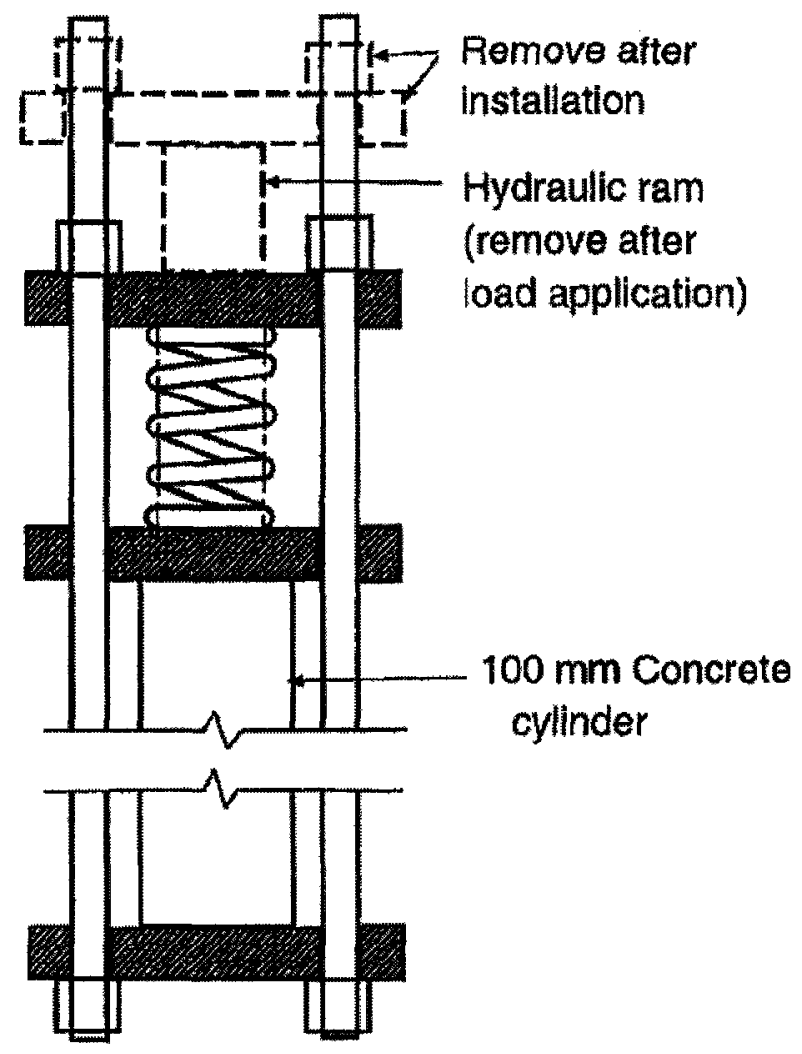

Figure 3.4. Creep and shrinkage loading device. (Cheung et al. 1997) 

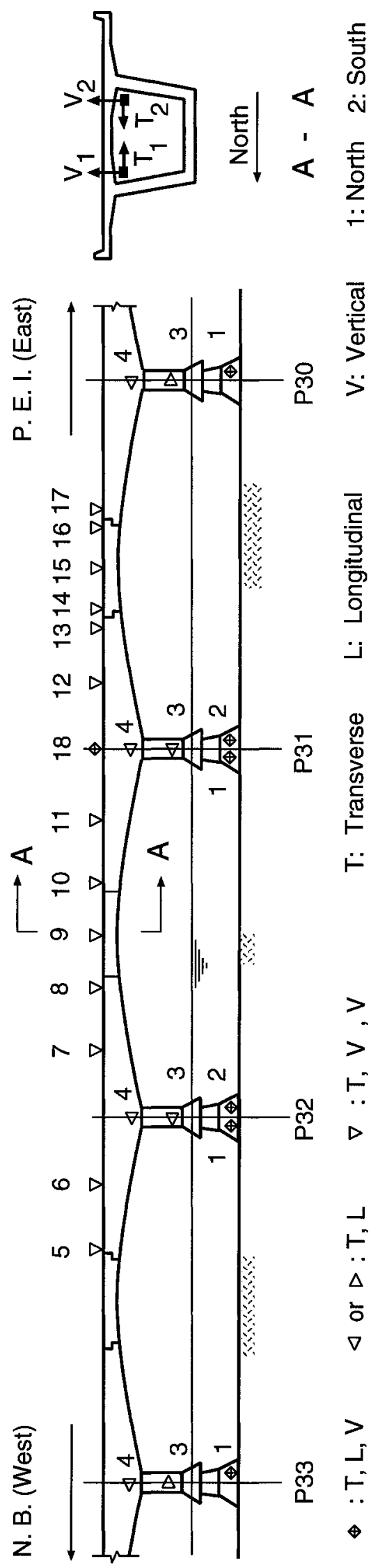

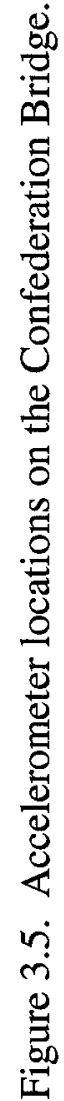



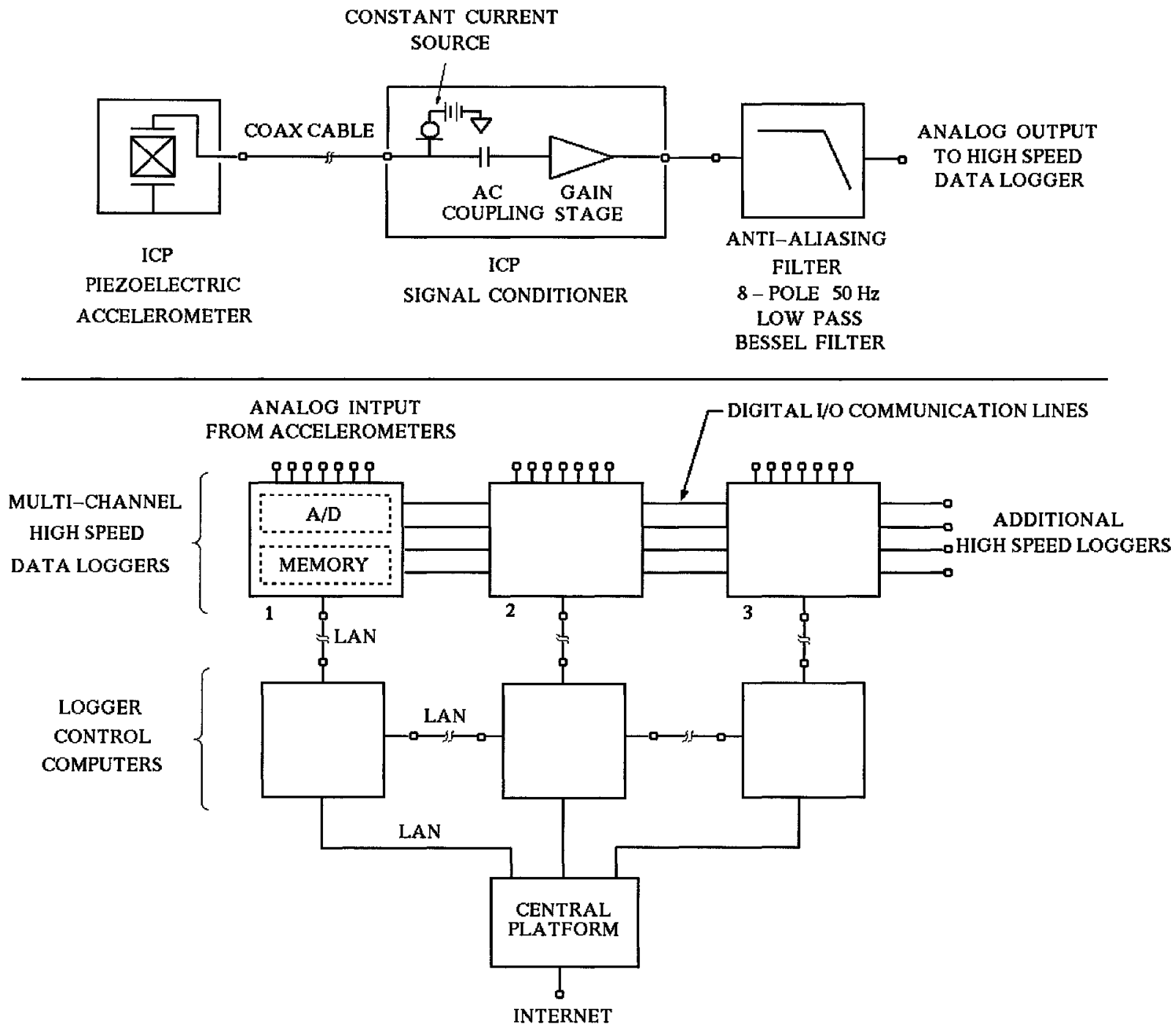

Figure 3.6. Data transmission schematic. Example show is for a signal captured from a piezo-electric accelerometer 


\section{Chapter 4}

\section{Processing of Dynamic Monitoring Data}

In Chapter 3, the collection of the structural response data of the Confederation Bridge monitoring project and transmission of the data to a remote site, in this case back to a data center at Carleton University, for archival and research, are described. In this chapter, the processing of the collected "raw" data is described. The purpose of the data processing tasks is to prepare the data for detailed complex analysis to extract engineering information embedded in the data volume, which is a process also known as data mining. Appropriate and accurate processing of the raw data are necessary because the accuracy and relevance of the engineering information extracted from the monitoring data are sensitive to the quality of the data, which in turn is affected by the processing procedures. The complex data analysis process and procedures are discussed in Chapter 
5. Anomalies in the raw data, such as signal drifting, offsets, noise contaminations, sampling errors, need to be repaired, minimized or eliminated. The consistent and accurate extractions of modal properties from the raw data after processing are critical for their use in future condition assessment of the monitored facility. Therefore it is highly important that the processing operations employ reliable numerical signal processing algorithms to correct any anomalies present in the data. Because of the very high volume of raw data collected by the vibration monitoring of the Confederation Bridge, the processing operations need to be rapid and automated for efficient handling of the data and information. Hence there is the obvious need for efficient, easy to use computer tools for rapid and automatic processing of the signal, and interface modules for easy handling and organization of the data and extracted information. This thesis concerns the development of such computer tools and modules in the form of a comprehensive computer software package platform for vibration-based structural health monitoring called SPLASH. The computer platform is specifically developed for monitoring of the Confederation Bridge. But the modules and interfaces described herein are developed in a modular form that can be easily adapted for other monitoring projects or applications. In this chapter, the characteristics of the "raw" vibration monitoring data of the Confederation Bridge are first discussed, followed by data management, pre-processing and processing of the raw data. The design and development of the data processing module with its sophisticated graphical user interface is discussed. Further data processing and analysis procedures by advanced system identification numerical techniques are presented in Chapter 5. 


\subsection{Dynamic Vibration Monitoring Data}

The monitoring data contained in data file packets transmitted to Carleton University via internet from the bridge site are output-only vibration responses of the Confederation Bridge to ambient excitation conditions. Typical ambient operational conditions include traffic, wind, current and ice floes. The signal is contained in data file packets in comma separated text format. The typical sampling rate of the records in the data files is $125 \mathrm{~Hz}$. Duration of a typical data file is set at 10 minutes to better capture the responses of lower vibration modes. For triggered event data, the data file include 30 second pre-trigger responses of the bridge.

Table 4.1 shows the header and data records of a sample data set of 0.48 seconds of data recording. The size of a typical 10 minute recording event, composed of three data sets from three separate data loggers, is approximately $48 \mathrm{MB}$. This corresponds to a total data volume of $7 \mathrm{~GB}$ per day if the monitoring system is operated under continuous data collection mode. The monitoring system can be set to operate in either continuous data collection mode or triggered mode. The current state of operation uses trigger recordings and collection of vibration data at a high sampling rate along with continuous statistical vibration data such as mean, maximum, minimum and standard deviation etc.

Figures 4.1 and 4.2 show typical 10 minute raw signal plots from locations 9V1 and 9T1 collected under predominantly wind and traffic conditions, respectively. Figures 4.3 and 4.4 show selected 40 second plots of the same data to better illustrate characteristics of the data signatures in the respective data sets. We can see that the vibration response excited by wind is dominated by low frequency behaviour with stationary characteristics, while response to traffic excitation of heavy trucks contains high frequency content and 
characteristics of rapid increase and decay of the vibration amplitude. Another observation worth noting is the maximum amplitudes recorded under the two excitation scenarios. Under strong wind excitations the maximum response amplitudes vary from 10 to $40 \mathrm{mV}$ while under strong traffic excitations the maximum response amplitudes range from 70 to $120 \mathrm{mV}$ (Note: for location 9, the QA-400 servo accelerometers have a $2.66 \mathrm{~V} / \mathrm{g}$ sensitivity). It is identified that the higher amplitude responses of traffic induced vibration are due to higher frequency sectional vibrations of the bridge girder. Typical vibration response measurements contain both structural responses to wind and traffic loading, and in winter also responses to ice floe impact and interaction with bridge piers.

Typical problems of the raw data which require processing to enhance the quality of the data are listed and discussed as follows:

- There can be a lack of time synchronization in the clocks of different data-loggers in the distributed monitoring system. Although triggered data collection occurs simultaneously at different data-loggers of the monitoring system, the time stamps contained in the separate data files from different loggers may differ slightly; this is caused by time differences in the data-logger clocks not properly synchronized.

- There may be small gaps in the data stream due to missed samples during high speed data acquisition. Data-loggers execute the data collection program at the CPU clock speed. A gap in the data records may occur when the data-logger processor is overburdened with additional operation commands beyond the normal data collection procedures, such as switching between different data collection modes as in the case of the end of the 30 second pre-trigger data buffer. 
- There can be duplication of data samples in small segments of the data, most likely caused by the slight out of exact synchronization of the data acquisition software which controls the data collection process with the logger control computer at the site.

- The data may exhibit a constant offset or linear trend of some accelerometer time history signals. Figures 4.1 and 4.2 clearly show some raw signal offsets.

- There can be high frequency noise contamination of the data, such as those caused by electrical noise at harmonics of the electrical frequency.

- Different loggers may be set to collect vibration response data at slightly different sampling rates for the same dynamic event in order to maximize efficient operation of the loggers.

- Data from a dynamic data set recorded by a given data logger may be partitioned into different data files, resulting in data files containing incomplete data set segments of duration shorter than specified. This is caused by the potential scenario when scheduled automatic collection process retrieves data from the logger's data table while the logger is sampling and recording. This causes data sets to be fractured and stored into different data files.

- Data from several triggered data sets may also be stored in a single data file.

All these problematic characteristics need to be identified and corrected automatically and rapidly before detailed data analysis for extraction of engineering information. The following sections discuss the step-by-step operations of the data management, preprocessing and processing algorithms. 


\subsection{Data Management and Pre-Processing Operations}

File management and pre-processing of the raw monitoring data are performed to repair or correct some of the above listed problems and to reorganize the data in suitable formats for subsequent data processing. The data processing and analysis tools are developed using Matlab (V6.5.0, Release 13) unless otherwise noted. The management and pre-processing modules organizes the data sets into appropriate formats for input to the processing engine and for archival. File management and pre-processing tasks include:

- Receiving of raw data folders. Under current triggered operation mode of the monitoring system, raw data files in compressed form from the bridge site data collection computers are transmitted to Ottawa-Carleton Bridge Research Institute (OCBRI) at Carleton University via internet. Separate compressed data sets are collected from each data loggers at the bridge, which are organized in separate folders. The number of data sets in each compressed folder depends on the frequency of transmission via internet and the set level of trigger threshold limit. If a high trigger threshold is set, a lower number of data sets are collected.

- Organization of data files. Each decompressed data file of .DAT format is renamed using a file naming system which identifies the time the data are collected, the datalogger that created the file and the trigger scenario (if available). For example a wind triggered data set recorded by logger 5 on November $13^{\text {th }} 2003$ at 7:32pm has a file name as 200311131932_log5_wind.DAT. This filename type is standard for file synchronization throughout the program. 
- Organization of complete data events. A complete data event contains 10 minute data recordings from all accelerometers. Accelerometer data signals are stored in three different files, which need to be synchronized and combined to form a complete data event.

- Conversion of data file format. The raw data files are given in comma separated ASCII format. An application developed in FORTRAN language has been written for converting the comma separated data file into an intermediate format, with extension .T referred here as t-file, and shown in Table 4.2. The first 6 columns of the t-file in Table 4.2 contain the time-stamp information of each record while the rest of the columns of Table 4.2 (columns 7 to 19) contain accelerometer signals connected to the respective data-logger (channels 1 to 13 of data-logger \# 1 in this case). The t-files are then converted to binary form and stored in a matrix in Microsoft Access Tables format with .mat extension for subsequent processing by the specifically developed computer platform written in Matlab.

- The separation of data sets. The difference between 'data set' and 'event' is explained here. A full data set is a 10 minute data recording from a single datalogger. A full event corresponds to 10 minute data recordings from all the dataloggers. A data event comprises of three data sets from three separate data-loggers. The algorithm searches linearly for large gaps in the data records by means of timestamps of the data records. A large gap is identified when a time step exceeds the sampling interval by a factor of 1000 . Once a large gap is identified, the algorithm truncates the remainder of the data signals contained in the data file into a separate data file for later processing. If the first part of the truncated data file constitutes a 
complete data set of about ten minutes duration, pre-processing of the data files continues. On the other hand, if only a data set segment is found, that is if the first part of the data file is less than ten minutes, the segment is stored in memory and can later be assembled into a full data set if the other matching segment is subsequently found.

- Assembly of matching data set segments. The algorithm searches all the stored data set segments and compares the start and end time-stamp of each looking for a match. When a match is found, the matching data sets are combined in proper chronological order. If the newly assembled data set is found to be of 10 minute duration, it is sent for processing. On the other hand, if the new data set is still less than 10 minutes it remains a data set segment stored in memory for further matching possibilities.

The pre-processing operation discussed here efficiently overcomes the common formatting difficulties associated with the data sets prior to signal processing. The preprocessing routine converts large amounts of chaotic data initially contained in three separate compressed folders of data files in ASCII format into sets of three mat-files containing data matrices of full 10 minute data sets which correspond to a full dynamic event recording. A copy of the pre-processed events is also sent to a database for archiving and back-up purposes. 


\subsection{Processing Operations}

The processing engine has as input a set of three pre-processed mat-files containing the raw signal in a matrix format. The signals contained in the matrices are still considered 'raw'; no processing operation has been performed on the data. The signal processing engine described in this section contains many algorithms which perform specific tasks as needed. The processing engine runs, when called upon, in loops for data events, data sets and data channels. Some operations are done on data sets as a whole while others are performed on individual channels. The different data processing operations and procedures carried out by the processing engine are described as follows:

- Purging of duplicate records. This algorithm loops through row vectors of the data matrix, starting with the earliest in chronological order, to find potential duplicating records. A potential duplicate record occurs when a sampling interval, calculated from the time-stamps, is found to be less than zero. The algorithm will then compare the potential duplicate records with earlier records to find a match. When a match is found the duplicate record is purged from the data set.

- Patching small data gaps. Small data gaps, where data samples are missing, are patched by a cubic polynomial interpolation algorithm using Piecewise Cubic Hermit Interpolating Polynomial (PCHIP). It operates on the column vectors of the signal matrix by taking a set number of samples, four in the present case, before and after the missing samples for interpolation, as shown by the sample data in Figure 4.5(a).

- De-trending of data. This algorithm loops through the column vectors of the raw data matrix of the accelerometer data recordings. The mean is calculated and 
removed from the signal, as shown in Figure 4.5(b). Another option for offsetremoval is by high-pass digital filtering of the signal to remove the DC or the nearzero frequency component.

- Scaling of data using calibration factors. Calibration factors are channel specific and are used to convert the acceleration readings from voltage into engineering units of $\mathrm{m} / \mathrm{s}^{2}$. A channel specification sheet which contains the accelerometer information, such as sensitivity and calibration factors, is input into the code syntax for use in this algorithm and throughout the processing engine. Scaling is done on a channel loop by multiplying each value of the specified column vector of the signal matrix by its specific calibration factor.

- Re-sampling of data to a common sampling rate. The data sets of a dynamic event are collected by multiple data-acquisition systems inside the bridge. The dataloggers of the acquisition system may sample at different rates. To synchronize the three signal matrices for output compatibility, the minimum sampling rate is determined and the two other data sets are subjected to a re-sampling operation. This re-sampling operation will lower the sampling rate of the signal at a ratio of integers $a / b$ established by the data set sampling intervals $[\Delta \mathrm{t} / \max (\Delta \mathrm{t})]$ using an interpolation algorithm to upsample the signal by the integer factor $b$ and then down-sampled by the integer factor $a$. This operation is carried out on each individual column vector of the signal matrix of a given data set. Any type of down-sampling operation inevitably results in aliasing of the signal and reduces the useful frequency range of the otherwise properly captured signals. Therefore signal filtering of the down-sampled data is needed. In this case a Finite Impulse 
Response (FIR) filter is used to low-pass filter the original signal vector to prevent aliasing (Section 2.2 discusses aliasing). Figure 4.6(a) shows a typical LeastSquares FIR filter design characteristics. The filter design characteristics vary depending on the ratio of integers $(a / b)$ and are automatically chosen based on operation requirements of the data. A new FIR filter is designed by the algorithm whenever a re-sampling operation is performed. Figure 4.6(b) shows an example of the magnitude response of a typical designed filter used for down-sampling a data set from a $125 \mathrm{~Hz}$ sampling rate to a $100 \mathrm{~Hz}$ sampling rate. The low-pass filter operates in time domain to remove any variations of the signal occurring at higher rate than specified by the pass-band frequency. Details of digital filters can be found in the reference from Hamming (1998).

- Decimation of data. The typical sampling rate for the Confederation Bridge monitoring project is currently set at $125 \mathrm{~Hz}$, while the frequency content of interest of the structure is primarily below $15 \mathrm{~Hz}$. This means processing and output computations of the data can be economized by reducing the amount of samples contained in the signal matrices. Decimation is very similar to re-sampling in that it includes both low-pass filtering and down-sampling operations. But, as opposed to the re-sampling algorithm, the decimation operation is always performed on the signals. The signal filter used in this case is an Infinite Impulse Response (IIR) filter. Figure 4.7(a) shows design characteristics of an 8th order Chebyshev type 1 IIR filter used in the processing engine. Figure 4.7(b) shows the magnitude response function and phase of the designed filter with a pass-band ripple $\left(A_{\text {pass }}\right)$ of $0.05 \mathrm{~dB}$. By decimating with a factor of three, the cutoff frequency ( $\left.F_{\text {pass }}\right)$ for our filter is 
determined by dividing the Nyquist frequency $\left(\mathrm{F}_{s} / 2\right)$ by the chosen decimation factor. A factor of 0.8 is added to obtain a conservative cut-off frequency of 16.7 Hz. This filter has a phase response function that is near-linear. Since "off-line" data are considered, signal distortions caused by the near-linear phase response function can be canceled out by filtering the signal in both directions in the timedomain. Once data is filtered, the down-sampled data is obtained by taking every $3^{\text {rd }}$ sample which significantly reduces the amount of data in the matrices without loss of pertinent information. Figure 4.8 shows an example of a decimated signal, notice the decimated signals attenuation near $16.7 \mathrm{~Hz}$ in the figure's inset.

- Un-filtering of channels. The QA-1200 servo-accelerometers at locations 3, 4 and 18 of pier 31 and at locations 3 and 4 of pier 32 were initially installed for the Ice floe monitoring. It was decided that the measurements from these locations would be valuable for the vibration monitoring project. A simple one-pole resistor/capacitor filter was fabricated with a $75 \mathrm{Kohm}$ resistor and $0.68 \mu \mathrm{F}$ capacitor and installed on-site at the above locations in order to reduce the output voltage signal from $99 \mathrm{~V} / \mathrm{g}$ to $10 \mathrm{v} / \mathrm{g}$. A discrete time transfer function is created in the algorithm with the same properties of the on-site R/C filter; the inverse of this transfer function is applied to data from these pier transducers. Figure 4.9 shows the result of un-filtering a pier channel.

- Double Integration. The signal is doubly integrated to obtain the displacement response of the structure using the cumulative trapezoidal numerical integration (CTNI) method. Precautions with low frequency content need to be taken when integrating a signal. Any DC component or near-zero frequency content are 
amplified during an integration operation. Therefore a high-pass filter is designed for use prior to the first and after each integration operation. Figure 4.10 shows the filter specifications of a Chebyshev type 2 IIR filter. The algorithm operations for double-integration are listed as follows:

- High-pass filtering of the acceleration data using the Chebyshev type II filter.

- Integrating the filtered acceleration data with the CTNI method yielding velocity signal.

- High-pass filtering the velocity with the Chebyshev filter to remove any constant offset from integration.

- Integrating the filtered velocity yielding using the CTNI method yielding displacement signal.

- High-pass filtering the displacement signal with the Chebyshev filter.

- Displacements are created mainly for the animation module; therefore to increase animation speed the data are further down-sampled by a factor of 4 without loss of visible response information. Figure 4.11 shows a typical signal subjected to the double integration algorithm.

- Three output matrices are created by a specialized algorithm and can be saved for use by the analysis and visualization modules:

- A structure of matrices containing the signal at different stages of the processing operation is saved for the visualization modules.

- A matrix containing the displacement signal is saved for the animation module. 
- A matrix containing the processed acceleration is saved for the operational modal analysis module.

\subsection{Processing Module with Graphical User Interface (GUI)}

In this section, the platform module that performs the data processing is described. The file sorting, preprocessing and processing engines are integrated into a single module and graphical user interface with most of the detailed operations occurring in the back-ground with detailed feedback on tasks performed provided on screen. Figure 4.12 shows the module's graphical user interface and Appendix $\mathrm{C}$ shows the module's organizational structure. The module has three run-time options:

- Data organization. Raw data from individual loggers are transmitted in a single compressed folder. The compressed folder may contain many triggered data sets or may be empty. These data files may contain one full data set (10 minutes) or more than one. Possibly, the data files may contain segments of data sets (less than 10 minutes). The 'Organize data' option will pre-process the data by: unzipping the folders, renaming the data files to a standard (timestamp_logger\#_triggermecanism), forming complete events, converting into proper data format and separating and matching data segments into 10 minute data sets, as needed.

- Processing the selected events. This option will call the processing function with the user-selected logger data files. These files must be named according to the aforementioned standard and must only contain one event. For example, user can 
select three logger data files that comprise a full dynamic event. The processing function receives the three files and performs all processing tasks and outputs the matrices containing information on all channels. If the user selects only one logger data file, the outputs created contain only information from the accelerometers of the chosen data-logger.

- Processing Real-Time. This option is set to run continuously in the background. The processing operation will be triggered by an incoming file or compressed folder. This file or compressed folder is automatically pre-processed as discussed in Section 4.2. Logger data files are then sent individually to the processing engine and the output column vectors are saved in a new file or are appended to an existing file if there is a time-stamp match in the output folder. The output algorithm searches the output folder for a matching file. If a matching file is found, the algorithm checks the data content in the found output matrix for data in the columns corresponding to the columns that are to be saved. The output displacement files are stored in a specific memory path where another module can detect the file and initiate the animation of bridge responses. Real-time animation is discussed in Chapter 6.

As mentioned in previous section, the processing module has three outputs. User specifies the output type(s) from the following:

- Displacement matrix. A matrix where the columns contain the displacement time history information at a single sensor location. Rows of this matrix correspond to displacement information of all channels at a specific instant in time. Time interval between rows is typically 0.096 seconds, up from original sampling rate of 0.08 
seconds because of decimation and down-sampling operations. The number of records for a ten minute event is reduced from 75000 for raw acceleration data to 6250 for displacement. This output matrix is used by the animation module to visualize response of bridge either in real-time or on an as-needed basis.

- Acceleration matrix. This matrix is used in the System Identification module for operational modal analysis. It contains the processed acceleration data. Columns contain acceleration time histories at each sensor location. The treatment of this data is discussed in Chapter 5.

- Structure of matrices containing data at all stages of processing. This structure of matrices is used by the data display module. It contains the following matrices

- Raw acceleration data (75000 records);

- De-trended and scaled acceleration data (75000 records);

- Patched acceleration data (75000 records);

- Decimated (filtered) acceleration data (25000 records);

- Velocity data (25000 records);

- Displacement data (6250 records). 
Table 4.1. Typical comma separated data file containing a data set from data-logger \#1

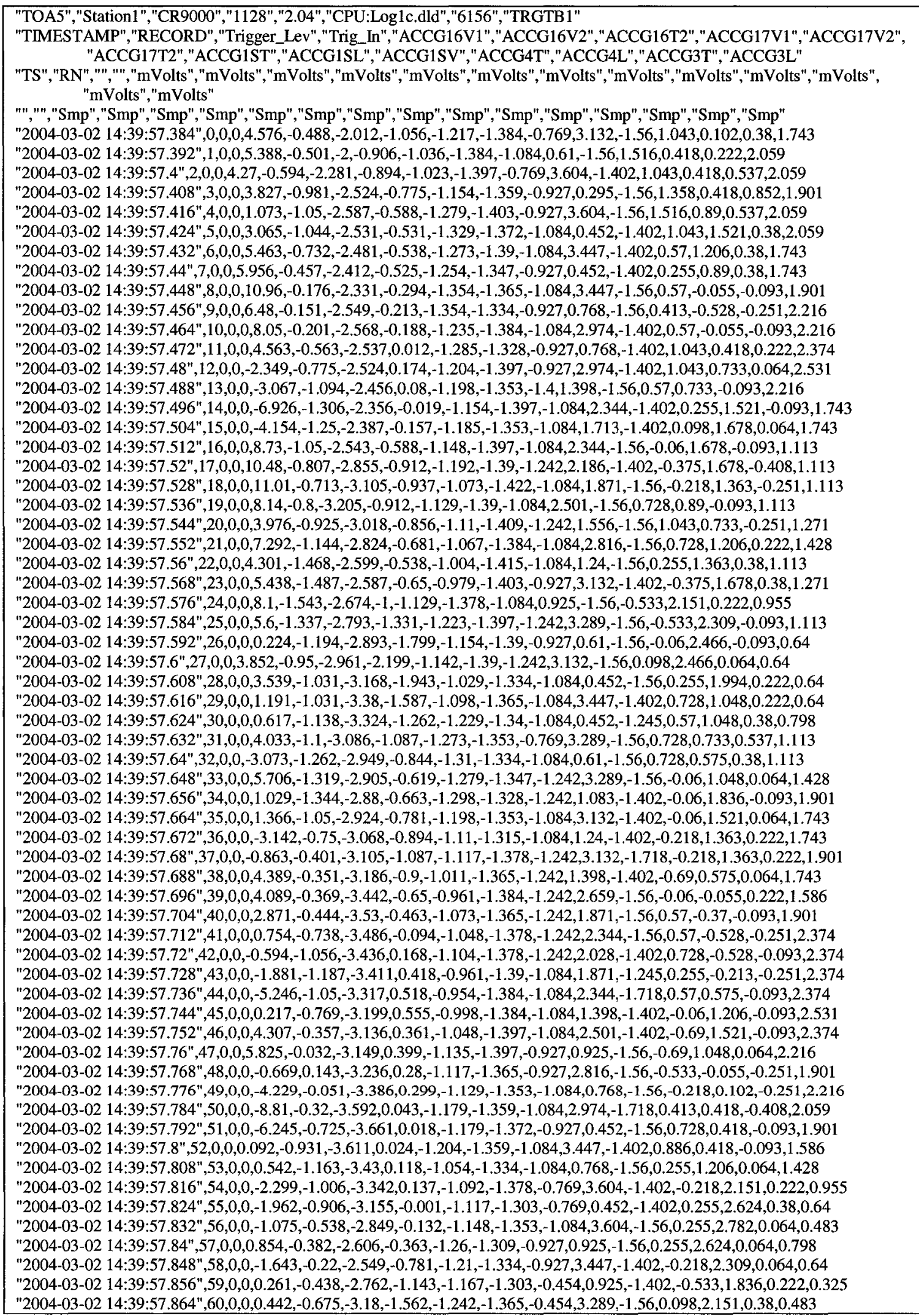


Table 4.2. Tab-delimitated intermediate data file format with .T extension (no header information)

\begin{tabular}{|c|c|c|c|c|c|c|c|c|c|c|c|c|c|}
\hline 20040302143957.384 & 4.57600 & -.48800 & -2.01200 & -1.05600 & -1.21700 & .1 .38400 & .76900 & 3.13200 & -1.56000 & 1.04300 & .10200 & .38000 & 1.74300 \\
\hline 20040302143957,392 & 5.38800 & -.50100 & $-2,00000$ & -.90600 & -1.03600 & -1.38400 & -1.08400 & .61000 & -1.56000 & 1.51600 & .41800 & .22200 & 2.05900 \\
\hline 20040302143957.4 & 4.27000 & .59400 & $-2,28100$ & -.89400 & -1.02300 & -1.39700 & .76900 & 3.60400 & .1 .40200 & 1.04300 & .41800 & .53700 & 2.05900 \\
\hline 20040302143957.408 & 3.82700 & -.98100 & -2.52400 & -.77500 & -1.15400 & -1.35900 & .92700 & .29500 & $-1,56000$ & 1.35800 & .41800 & .85200 & 1.90100 \\
\hline 20040302143957.416 & 1.07300 & -1.05000 & -2.58700 & -.58800 & $-1,27900$ & -1.40300 & -.92700 & 3.60400 & -1.56000 & 1.51600 & .89000 & .53700 & 2.05900 \\
\hline 20040302143957.424 & 3.06500 & -1.04400 & -2.53100 & .53100 & $-1,32900$ & -1.37200 & -1.08400 & .45200 & -1.40200 & 1.04300 & 1.52100 & .38000 & 2.05900 \\
\hline 20040302143957.432 & 5.46300 & -.73200 & -2.48100 & -.53800 & -1.27300 & -1.39000 & -1.08400 & 3.44700 & -1.40200 & .57000 & 1.20600 & .38000 & 1.74300 \\
\hline 20040302143957.44 & 5.95600 & -.45700 & -2.41200 & -.52500 & -1.25400 & -1.34700 & .92700 & .45200 & -1.40200 & .25500 & .89000 & .38000 & 1.74300 \\
\hline 20040302143957.448 & 10.96000 & -.17600 & -2.33100 & -29400 & -1.35400 & -1.36500 & -1.08400 & 3.44700 & .1 .56000 & .57000 & -.05500 & -.09300 & 1.90100 \\
\hline 20040302143957.456 & 6.48000 & -.15100 & -2.54900 &. .21300 & -1.35400 & -1.33400 & -.92700 & .76800 & -1.56000 & .41300 & -.52800 & -.25100 & 2.21600 \\
\hline 20040302143957.464 & 8.05000 & -.20100 & $\cdot 2.56800$ & .18800 & -1.23500 & $-1,38400$ & -1.08400 & 2.97400 & -1.40200 & .57000 & .05500 & .09300 & 2.21600 \\
\hline 20040302143957.472 & 4.56300 & .56300 & -2.53700 & .01200 & -1.28500 & -1.32800 & -.92700 & .76800 & -1.40200 & 1.04300 & .41800 & .22200 & 2,37400 \\
\hline 20040302143957.48 & -2.34900 & -.77500 & -2.52400 & .17400 & -1.20400 & -1.39700 & -.92700 & 2.97400 & -1.40200 & 1.04300 & .73300 & .06400 & 2.53100 \\
\hline 20040302143957.488 & -3.06700 & -1.09400 & -2.45600 & .08000 & -1.19800 & -1.35300 & -1.40000 & 1.39800 & -1.56000 & .57000 & .73300 & -.09300 & 2.21600 \\
\hline 20040302143957.496 & -6.92600 & -1.30600 & -2.35600 & -.01900 & -1.15400 & .139700 & $-1,08400$ & 2,34400 & $-1,40200$ & .25500 & 1.52100 & -.09300 & 1.74300 \\
\hline 20040302143957.504 & -4.15400 & -1.25000 & -2.38700 & -.15700 & -1.18500 & -1.35300 & $-1,08400$ & 1.71300 & -1.40200 & .09800 & 1.67800 & .06400 & 1.74300 \\
\hline 20040302143957.512 & 8.73000 & -1.05000 & -2.54300 & -.58800 & -1.14800 & -1.39700 & -1.08400 & 2.34400 & -1.56000 & -.06000 & 1.67800 & .09300 & 1.11300 \\
\hline 20040302143957.52 & 10.48000 & -.80700 & $-2,85500$ & -.91200 & $-1,19200$ & -1.39000 & $-1,24200$ & 2.18600 & -1.40200 & .37500 & 1.67800 & -.40800 & 1.11300 \\
\hline 20040302143957.528 & 11.01000 & -.71300 & -3.10500 & -.93700 & -1.07300 & -1.42200 & -1.08400 & 1.87100 & -1.56000 & .21800 & 1.36300 & -.25100 & 1.11300 \\
\hline 20040302143957.536 & 8.14000 & -.80000 & -3.20500 & -.91200 & -1.12900 & -1.39000 & $-1,08400$ & 2.50100 & -1.56000 & .72800 & .89000 & -.09300 & 1.11300 \\
\hline 20040302143957.544 & 3.97600 &,- 92500 & -3.01800 & .85600 & -1.11000 & -1.40900 & $-1,24200$ & 1.55600 & -1.56000 & 1.04300 & .73300 & .25100 & 1.27100 \\
\hline 20040302143957.552 & 7.29200 & -1.14400 & -2.82400 & -.68100 & -1.06700 & .1 .38400 & -1.08400 & 2.81600 & -1.56000 & .72800 & 1.20600 & .22200 & 1.42800 \\
\hline 20040302143957.56 & 4.30100 & -1.46800 & -2.59900 & -.53800 & $-1,00400$ & -1.41500 & -1.08400 & 1.24000 & -1.56000 & .25500 & 1.36300 & .38000 & 1.11300 \\
\hline 20040302143957.568 & 5.43800 & $-1,48700$ & -2.58700 & .65000 & .97900 & $-1,40300$ & .92700 & 3.13200 & -1.40200 & -.37500 & 1.67800 & .38000 & 1.27100 \\
\hline 20040302143957.576 & 8.10000 & -1.54300 & -2.67400 & -1.00000 & -1.12900 & -1.37800 & -1.08400 & .92500 & -1.56000 & .53300 & 2.15100 & .22200 & .95500 \\
\hline 2004 & 5.60000 & -1.33700 & -2.79300 & -1.33100 & -1.22300 & -1.39700 & -1.24200 & 3.28900 & -1.56000 & -.53300 & 2.30900 & -.09300 & 1.11300 \\
\hline 20040302143957.592 & .22400 & -1.19400 & -2.89300 & -1.79900 & -1.15400 & -1.39000 & -.92700 & .61000 & -1.56000 & -.06000 & 2.46600 & -.09300 & .64000 \\
\hline 20040302143957.6 & 3.85200 & .95000 & -2.96100 & -2.19900 & -1.14200 & -1.39000 & $-1,24200$ & 3.13200 & -1.56000 & .09800 & 2.46600 & .06400 & .64000 \\
\hline 20040302143957.608 & 3.53900 & -1.03100 & -3.16800 & -1.94300 & -1.02900 & -1.33400 & $-1,08400$ & .45200 & -1.56000 & .25500 & 1.99400 & .22200 & .64000 \\
\hline 20040302143957.616 & 1.19100 & -1.03100 & -3.38000 & -1.58700 & -1.09800 & -1.36500 & -1.08400 & 3.44700 & -1.40200 & .72800 & 1.04800 & .22200 & .64000 \\
\hline 20040302143957.624 & .61700 & -1.13800 & -3.32400 & -1.26200 & -1.22900 & -1.34000 & -1.08400 & .45200 & $-1,24500$ & .57000 & 1.04800 & .38000 & .79800 \\
\hline 20040302143957.632 & 4.03300 & -1.10000 & -3.08600 & $-1,08700$ & -1.27300 & $-1,35300$ & .76900 & 3.28900 & -1.56000 & .72800 & .73300 & .53700 & 1.11300 \\
\hline 20040302143957.64 & -3.07300 & -1.26200 & -2.94900 & -.84400 & -1.31000 & -1.33400 & $-1,08400$ & .61000 & -1.56000 & .72800 & .57500 & .38000 & 1.11300 \\
\hline 20040302143957.648 & 5.70600 & -1.31900 & -2.90500 & -.61900 & -1.27900 & -1.34700 & -1.24200 & 3.28900 & -1.56000 & .06000 & 1.04800 & .06400 & 1.42800 \\
\hline 1.656 & 1.02900 & -1.34400 & -2.88000 & -.66300 & $-1,29800$ & -1.32800 & -1.24200 & 1.08300 & -1.40200 & .06000 & 1.83600 & .09300 & 1.90100 \\
\hline 20040302143957.664 & 1.36600 & -1.05000 & -2.92400 & .78100 & -1.19800 & -1.35300 & -1.08400 & 3.13200 & -1.40200 & -.06000 & 1.52100 & .06400 & 1.74300 \\
\hline 20040302143957.672 & $-3,14200$ & -.75000 & -3.06800 & -.89400 & -1.11000 & -1.31500 & $-1,08400$ & 1.24000 & $-1,40200$ & -.21800 & 1.36300 & .22200 & 1.74300 \\
\hline 20040302143957.68 & -.86300 & -.40100 & -3.10500 & -1.08700 & -1.11700 & -1.37800 & -1.24200 & 3.13200 & -1.71800 & -.21800 & 1.36300 & .22200 & 1.90100 \\
\hline 20040302143957.688 & 4.38900 & -.35100 & -3.18600 & -.90000 & -1.01100 & -1.36500 & -1.24200 & 1.39800 & -1.40200 & -.69000 & .57500 & .06400 & 1.74300 \\
\hline 20040302143957.696 & 4.08900 & -.36900 & -3.44200 & -.65000 & -96100 & -1.38400 & -1.24200 & 2.65900 & -1.56000 & -06000 & -05500 & .22200 & 1.58600 \\
\hline 20040302143957.704 & 2.87100 &. .44400 & -3.53000 & -.46300 & -1.07300 & -1.36500 & -1.24200 & 1.87100 & -1.56000 & .57000 & -.37000 & -.09300 & 1.90100 \\
\hline 20040302143957.712 & .75400 & -.73800 & -3.48600 & -.09400 & $-1,04800$ & -1.37800 & -1.24200 & 2.34400 & -1.56000 & .57000 & -.52800 & -.25100 & 2.37400 \\
\hline 20040302143957.72 & -.59400 & -1.05600 & -3.43600 & .16800 & -1.10400 & -1.37800 & -1.24200 & 2.02800 & -1.40200 & .72800 &. .52800 & .09300 & 2.37400 \\
\hline 20040302143957.728 & $-1,88100$ & -1.18700 & -3.41100 & .41800 & -96100 & -1.39000 & -1.08400 & 1.87100 & .1 .24500 & .25500 & -.21300 & -.25100 & 2.37400 \\
\hline 20040302143957.736 & -5.24600 & -1.05000 & -3.31700 & .51800 & -.95400 & -1.38400 & -1.08400 & 2.34400 & -1.71800 & .57000 & .57500 & .09300 & 2.37400 \\
\hline 20040302143957.744 & .21700 & -76900 & -3.19900 & .55500 & -99800 & -1.38400 & -1.08400 & 1.39800 & -1.40200 & -.06000 & 1.20600 & .09300 & 2.53100 \\
\hline 20040302143957.752 & 4.30700 & -.35700 & -3.13600 & .36100 & -1.04800 & $-1,39700$ & -1.08400 & 2.50100 & -1.40200 & -69000 & 1.52100 & -.09300 & 2.37400 \\
\hline 20040302143957.76 & 5.82500 & -.03200 & -3.14900 & .39900 & -1.13500 & -1.39700 &,- 92700 & .92500 & -1.56000 & .69000 & 1.04800 & .06400 & 2.21600 \\
\hline 20040302143957.768 & .66900 & .14300 & -3.23600 & .28000 & -1.11700 & -1.36500 & -.92700 & 2.81600 & -1.56000 & -.53300 & -.05500 & -.25100 & 1.90100 \\
\hline 20040302143957.776 & -4.22900 & -.05100 & -3.38600 & .29900 & -1.12900 & -1.35300 & -1.08400 & .76800 & -1.56000 & -.21800 & .10200 & -.25100 & 2,21600 \\
\hline 20040302143957.784 & -8.81000 & -.32000 & -3.59200 & .04300 & $-1,17900$ & $-1,35900$ & -1.08400 & 2.97400 & -1.71800 & .41300 & .41800 & -.40800 & 2.05900 \\
\hline 20040302143957.792 & -6.24500 & -.72500 & -3.66100 & .01800 & -1.17900 & -1.37200 & -.92700 & .45200 & -1.56000 & .72800 & .41800 & .09300 & 1.90100 \\
\hline 20040302143957.8 & .09200 & -.93100 & -3.61100 & .02400 & -1.20400 & -1.35900 & -1.08400 & 3.44700 & -1.40200 & .88600 & .41800 & -.09300 & 1.58600 \\
\hline 20040302143957.808 & .54200 & -1.16300 & -3.43000 & .11800 & -1.05400 & -1.33400 & -1.08400 & .76800 & -1.56000 & .25500 & 1.20600 & .06400 & 1.42800 \\
\hline 20040302143957.816 & -2.29900 & -1.00600 & -3.34200 & .13700 & -1.09200 & -1.37800 & -76900 & 3.60400 & -1.40200 & -.21800 & 2.15100 & .22200 & .95500 \\
\hline 20040302143957.824 & -1.96200 & .90600 & -3.15500 & -.00100 & -1.11700 & .1 .30300 & .76900 & .45200 & -1.40200 & .25500 & 2.62400 & .38000 & .64000 \\
\hline 20040302143957.832 & -1.07500 & -.53800 & $-2,84900$ & -.13200 & -1.14800 & -1.35300 & -1.08400 & 3.60400 & -1.56000 & .25500 & 2.78200 & .06400 & .48300 \\
\hline 20040302143957.84 & .85400 & -.38200 & -2.60600 & -.36300 & -1.26000 & -1.30900 & -.92700 & .92500 & -1.56000 & .25500 & 2.62400 & .06400 & .79800 \\
\hline 20040302143957.848 & -1.64300 & -.22000 & -2.54900 & -.78100 & -1.21000 & -1.33400 & .92700 & 3.44700 & -1.40200 & -.21800 & 2.30900 & .06400 & .64000 \\
\hline 20040302143957.856 & .26100 & -.43800 & -2.76200 & -1.14300 & -1.16700 & -1.30300 & -.45400 & .92500 & -1.40200 & -.53300 & 1.83600 & .22200 & .32500 \\
\hline 20040302143957.864 & .44200 & .67500 & -3.18000 & -1.56200 & -1.24200 & -1.36500 & -.45400 & 3.28900 & -1.56000 & .09800 & 2.15100 & .38000 & .48300 \\
\hline
\end{tabular}




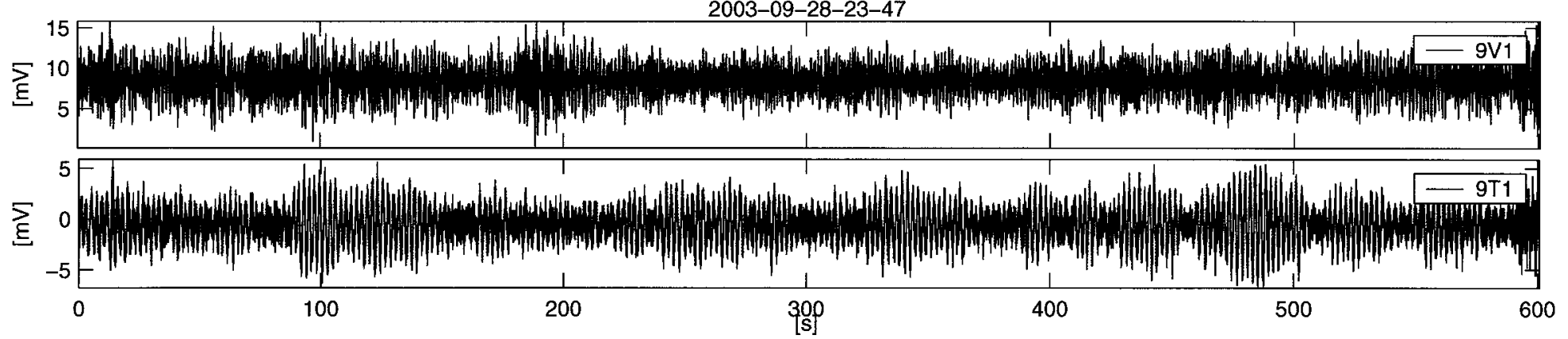

2003-09-29-02-47

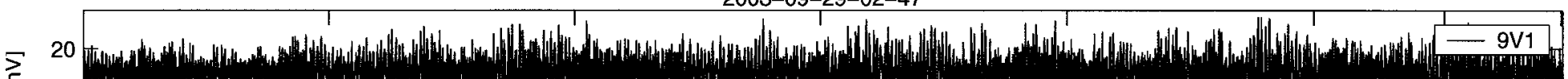

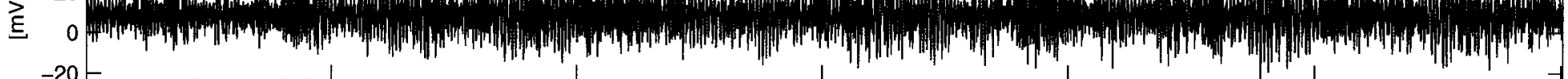

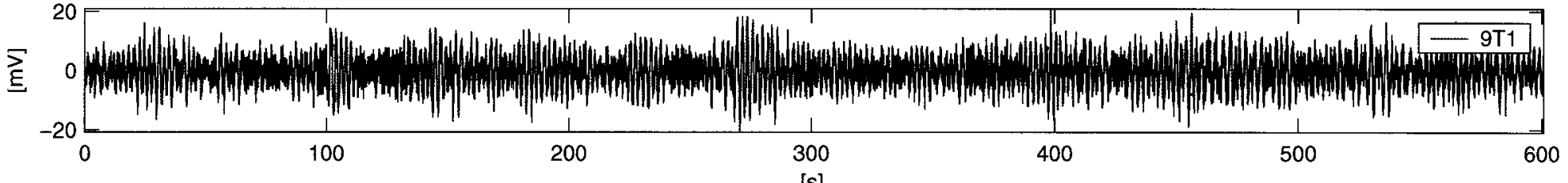
[s]

2003-11-29-13-16

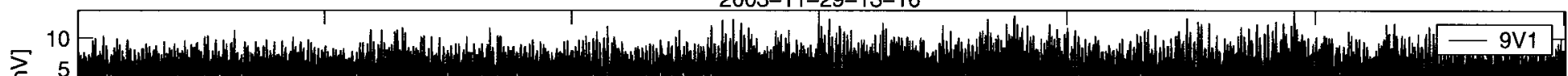
$\sum_{5} \begin{gathered}5 \\ 0\end{gathered}$ $\sum_{0}$
0
-5

Figure 4.1. Typical response monitoring data due to wind excitation. Full duration data set of 10 minutes. 
2003-07-16-17-26
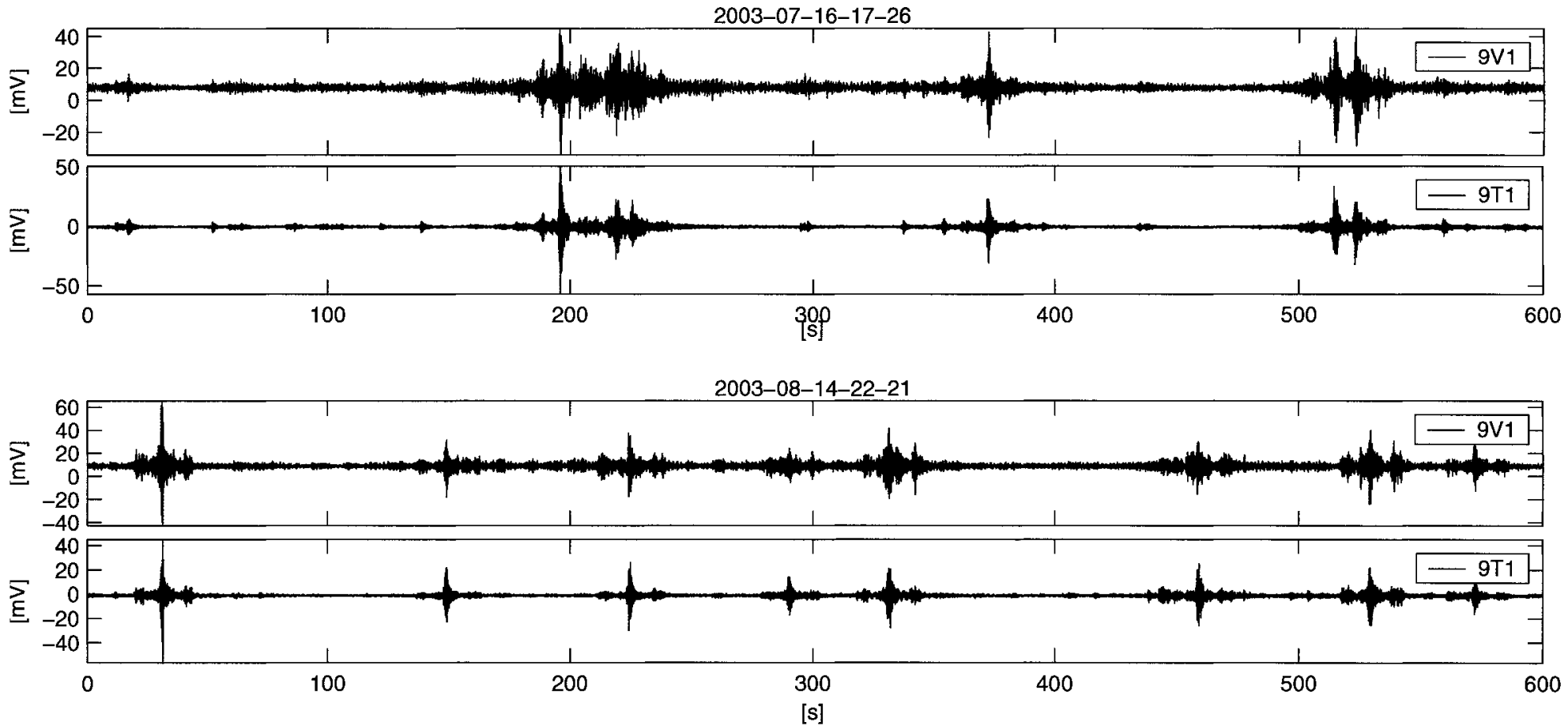

2003-09-26-00-30

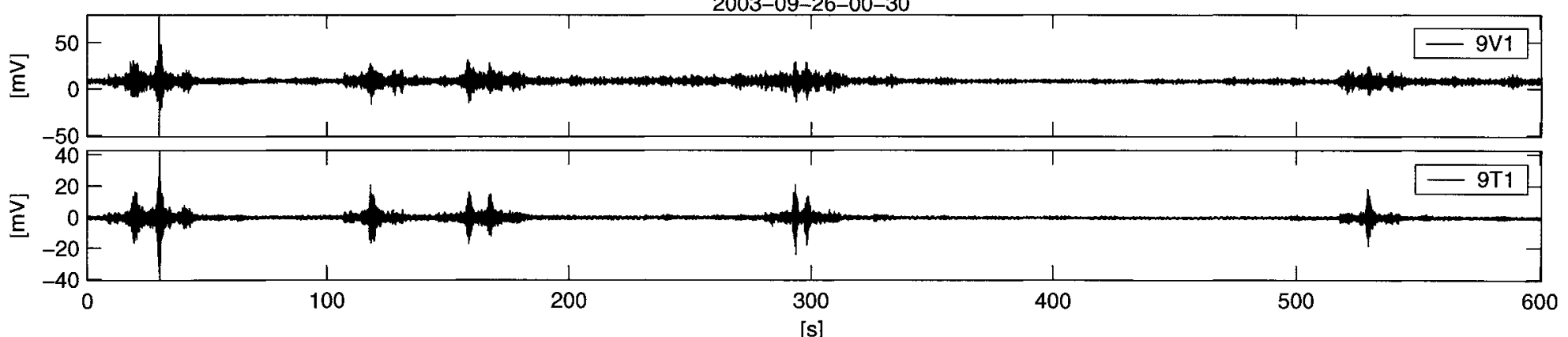

Figure 4.2. Typical response monitoring data due to traffic excitation. Full duration data set of 10 minutes. 


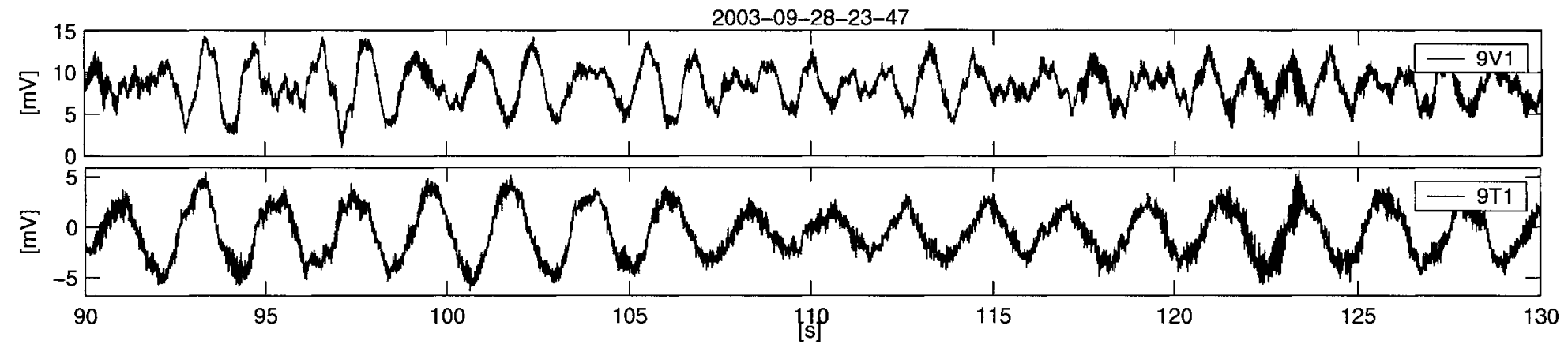

$\sum_{0}^{20}{ }_{-20}^{m}$

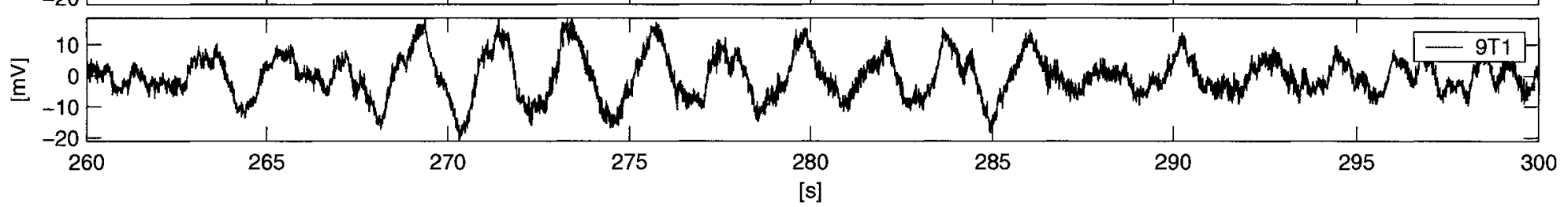
2003-11-29-13-16

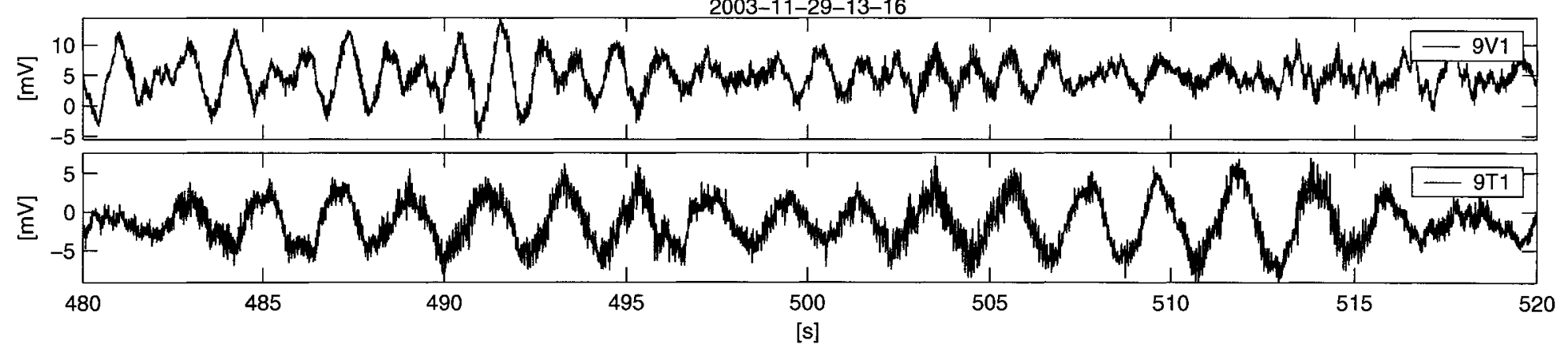

Figure 4.3. Typical response monitoring data due to wind excitation (40 seconds). 


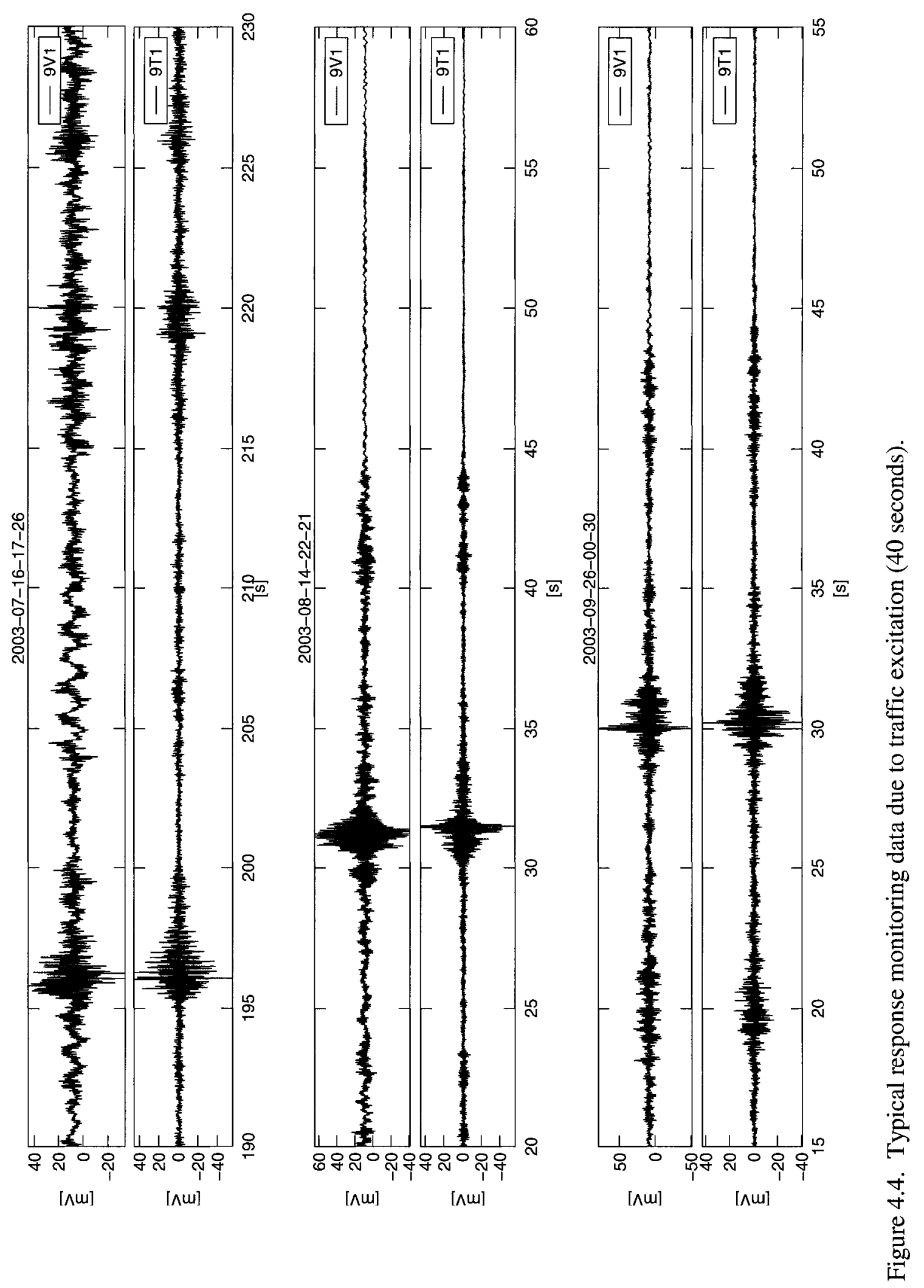



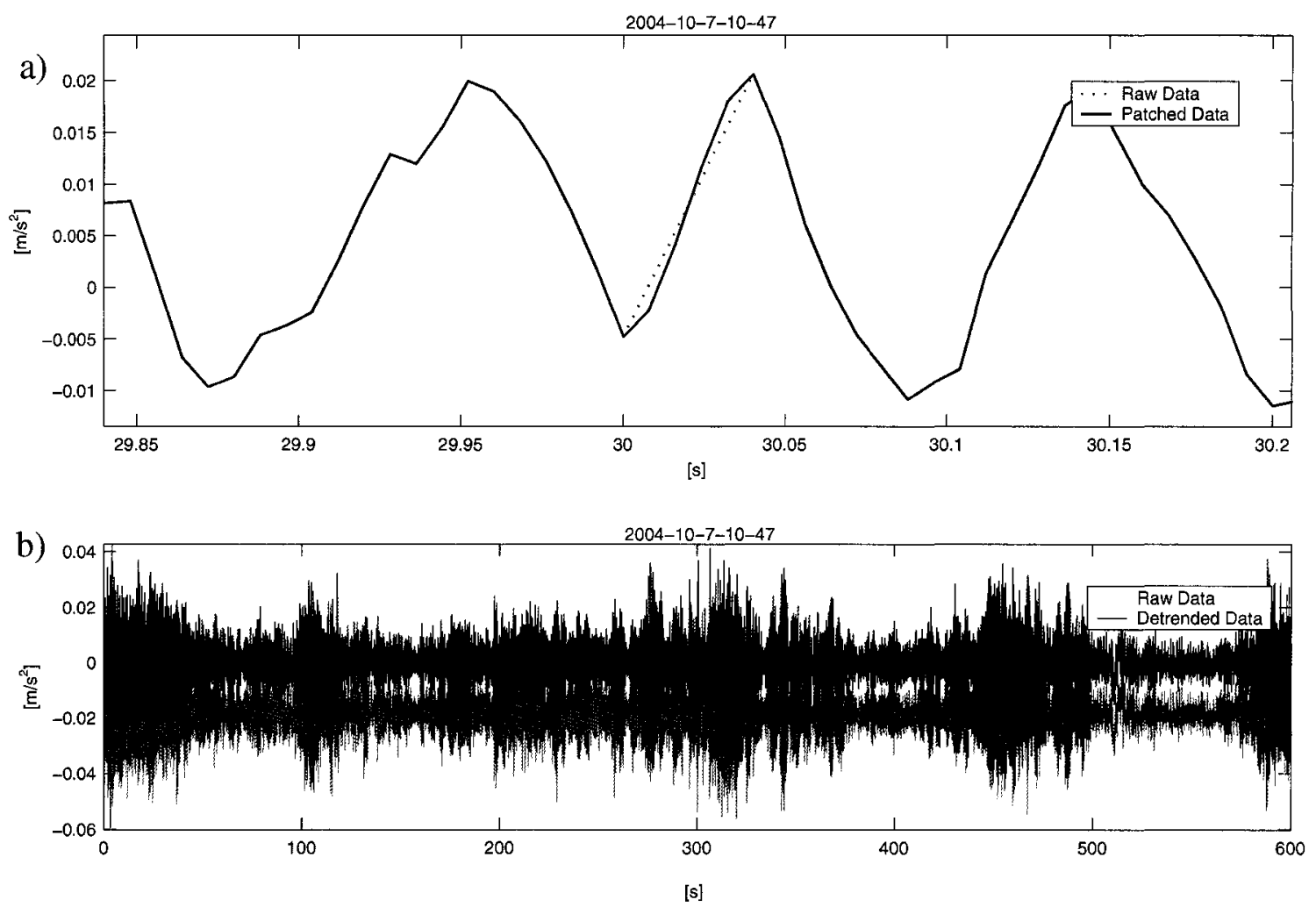

Figure 4.5. Common data characteristics and corrections. a) Data patching b) Signal offset removal. 

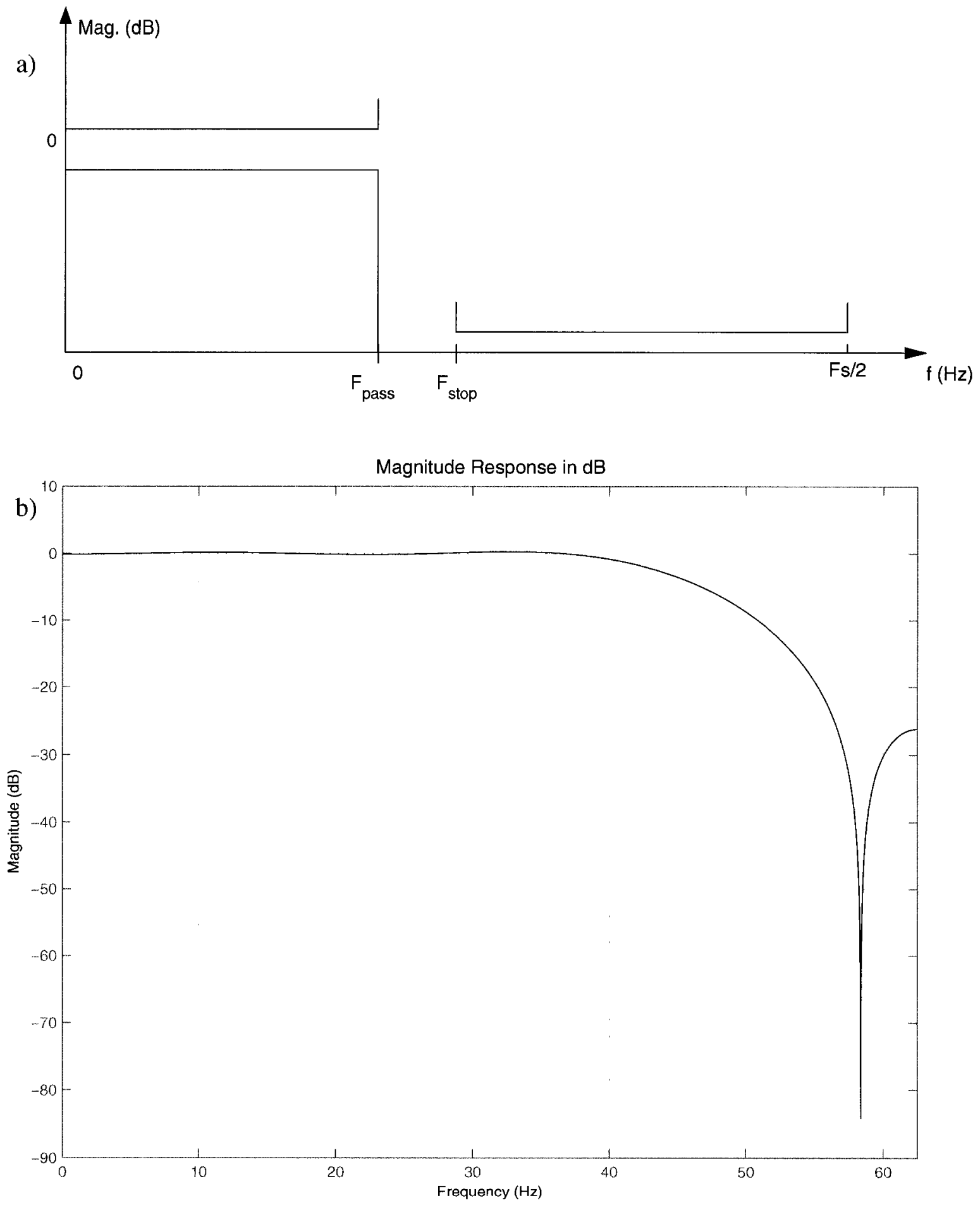

Figure 4.6. Finite Impulse Response (FIR) low-pass filter used in the re-sampling operation to prevent anti-aliasing. a) Design characteristics for a Least-Squares FIR filter b) Magnitude response of an LSFIR designed filter. 
a)
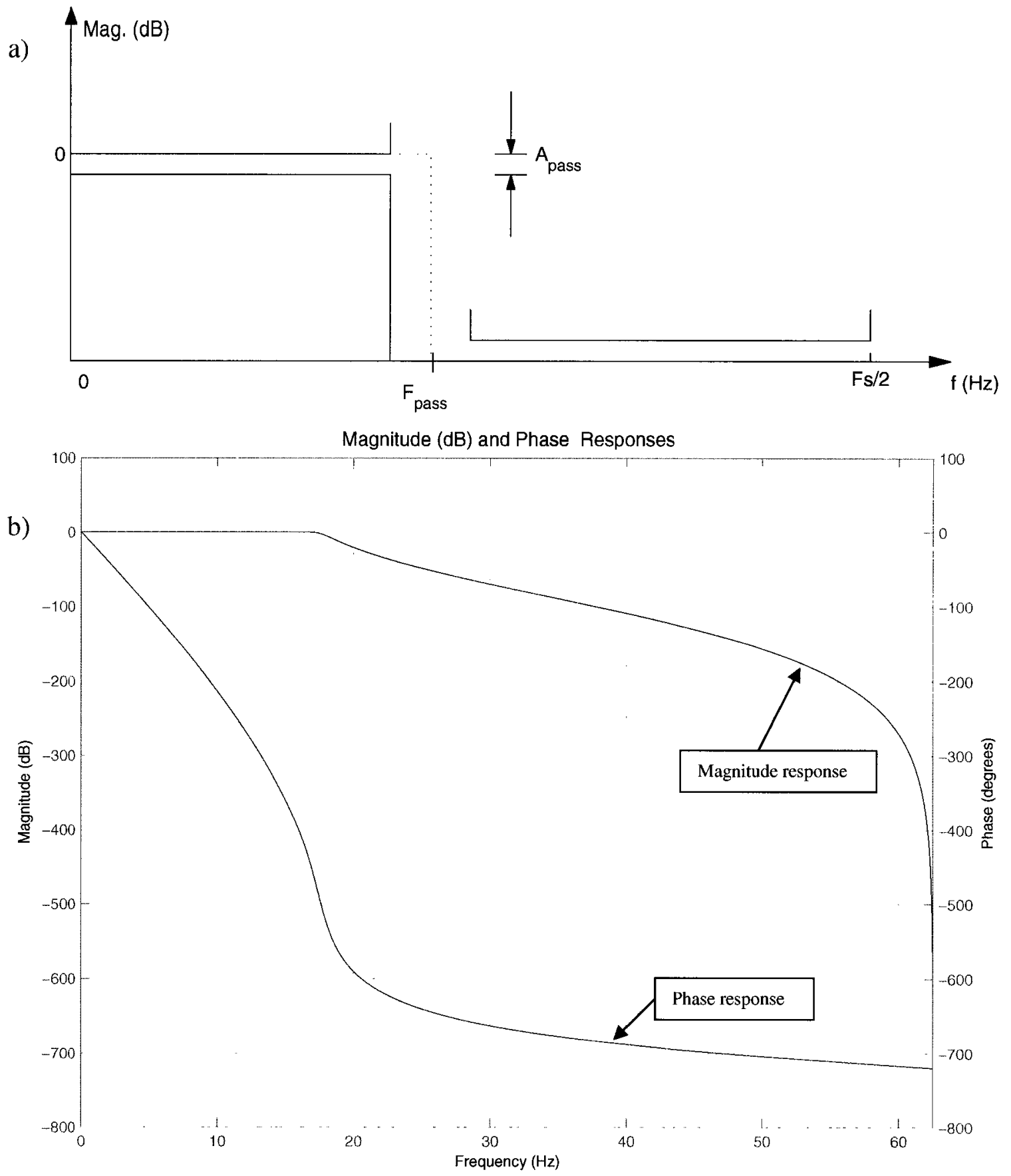

Figure 4.7. Infinite Impulse Response (IIR) low-pass filter used in decimation algorithm. a) Filter design characteristics of an $8^{\text {th }}$ order Chebyshev type I with cut-off frequency $\left(\mathrm{F}_{\text {pass }}\right)$ of $16.7 \mathrm{~Hz}$ and pass-band ripple $\left(\mathrm{A}_{\text {pass }}\right)$ of $0.05 \mathrm{~dB}$. b) Filter's magnitude response in $\mathrm{dB}$ (blue) and phase in degrees (green). 


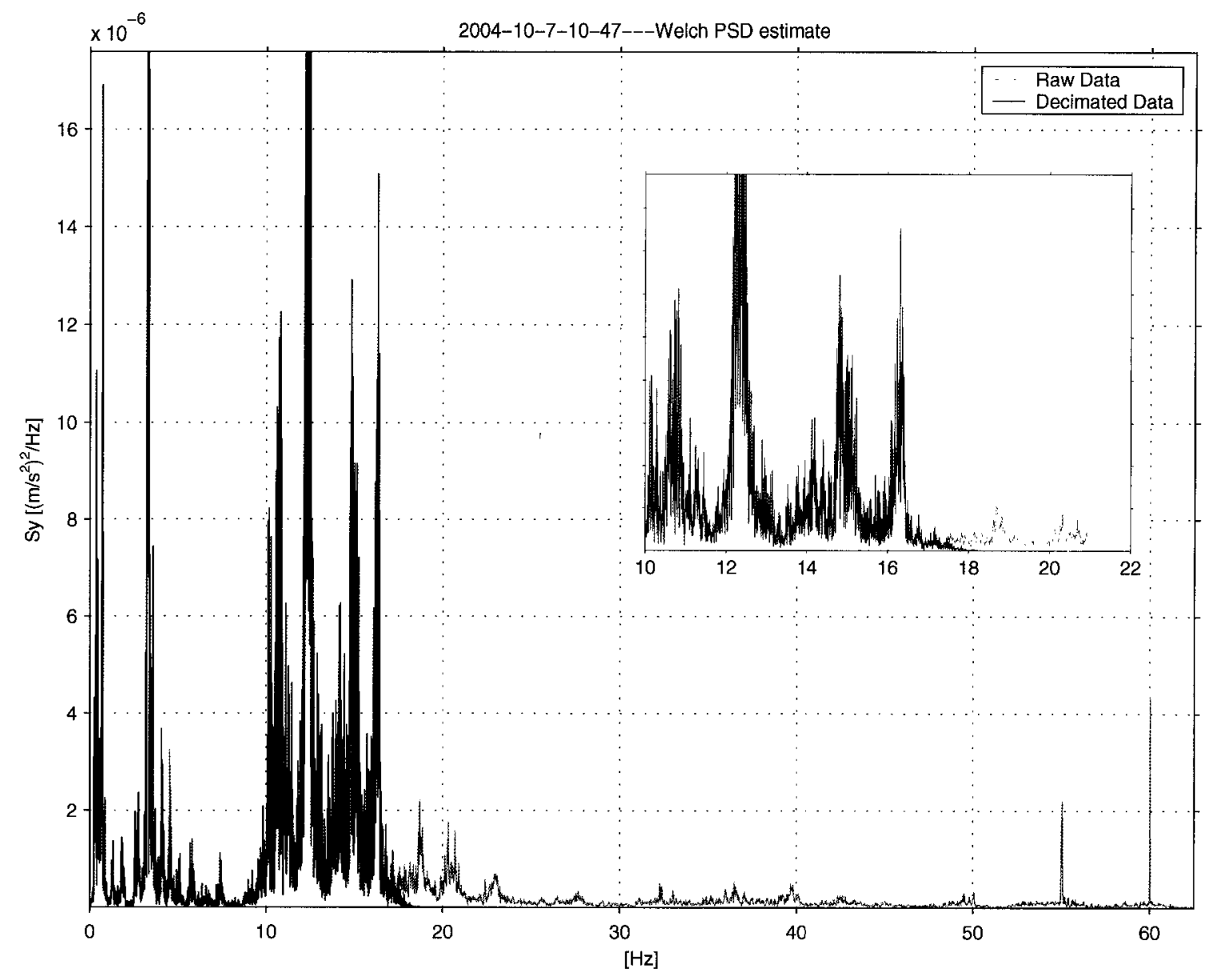

Figure 4.8. Example of a decimated signal with removal of high frequency noise contaminations at location $12 \mathrm{~T}_{2}$ (electrical harmonics). Inset shows attenuation of energy content at $16.7 \mathrm{~Hz}$. 


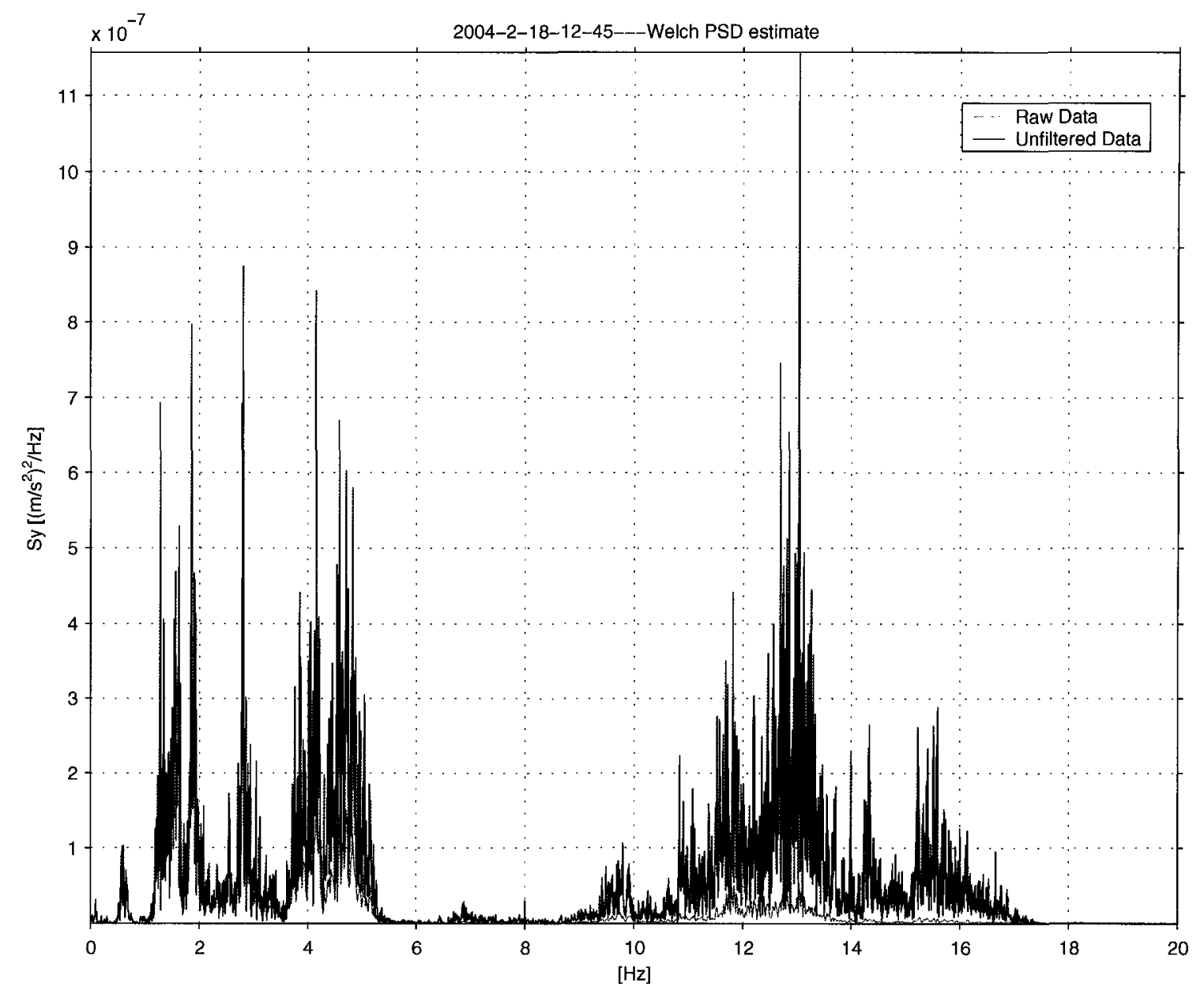

Figure 4.9. Example of an un-filtered signal at location 18T. 
a)
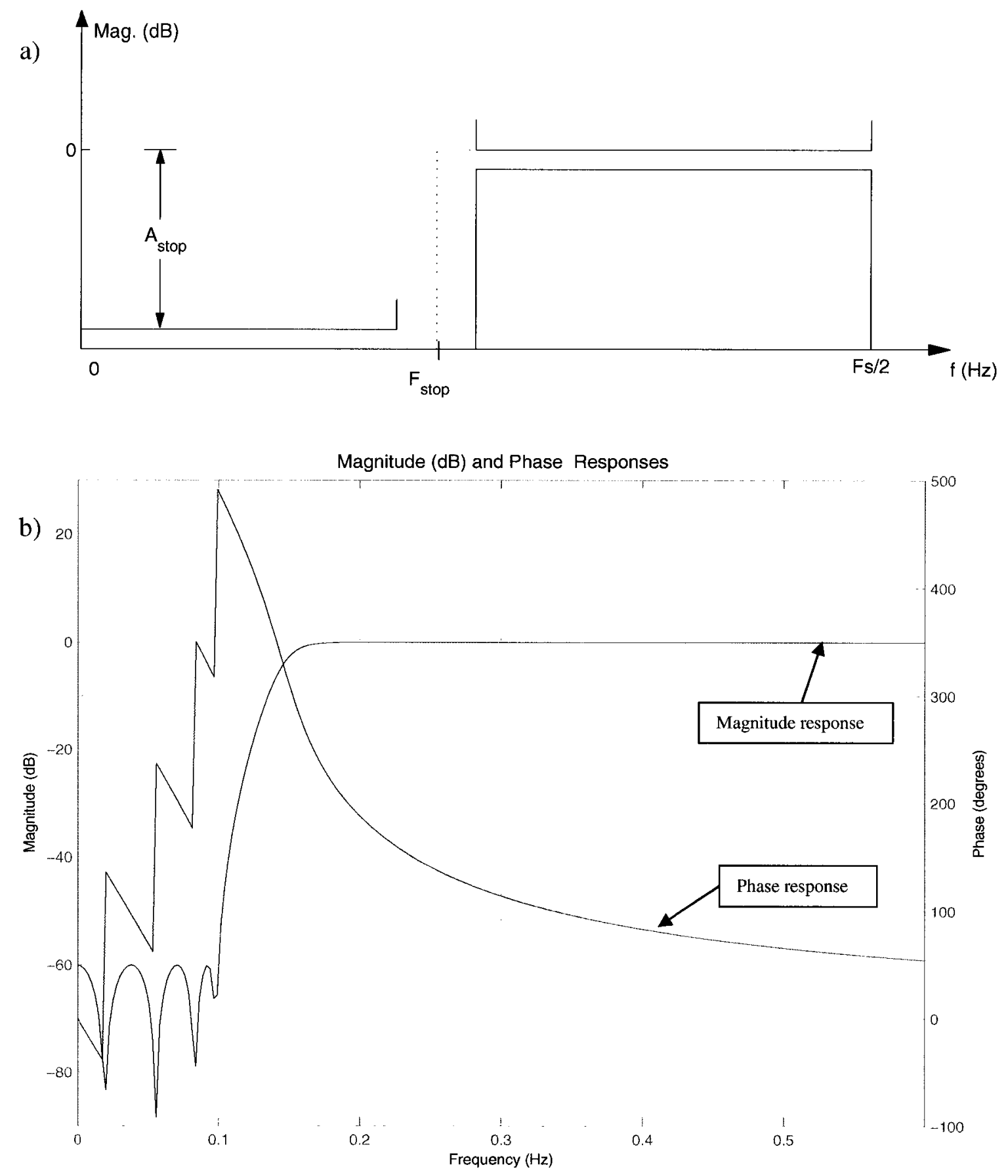

Figure 4.10. Infinite Impulse Response (IIR) high-pass filter used in the doubleintegration algorithm. a) Filter design characteristics of a $6^{\text {th }}$ order Chebyshev type II with cut-off frequency $\left(\mathrm{F}_{\text {stop }}\right)$ of $0.1 \mathrm{~Hz}$ and stop-band attenuation $\left(\mathrm{A}_{\text {stop }}\right)$ of $60 \mathrm{~dB}$. b) Filter's magnitude response in $\mathrm{dB}$ (blue) and phase in degrees (green). 

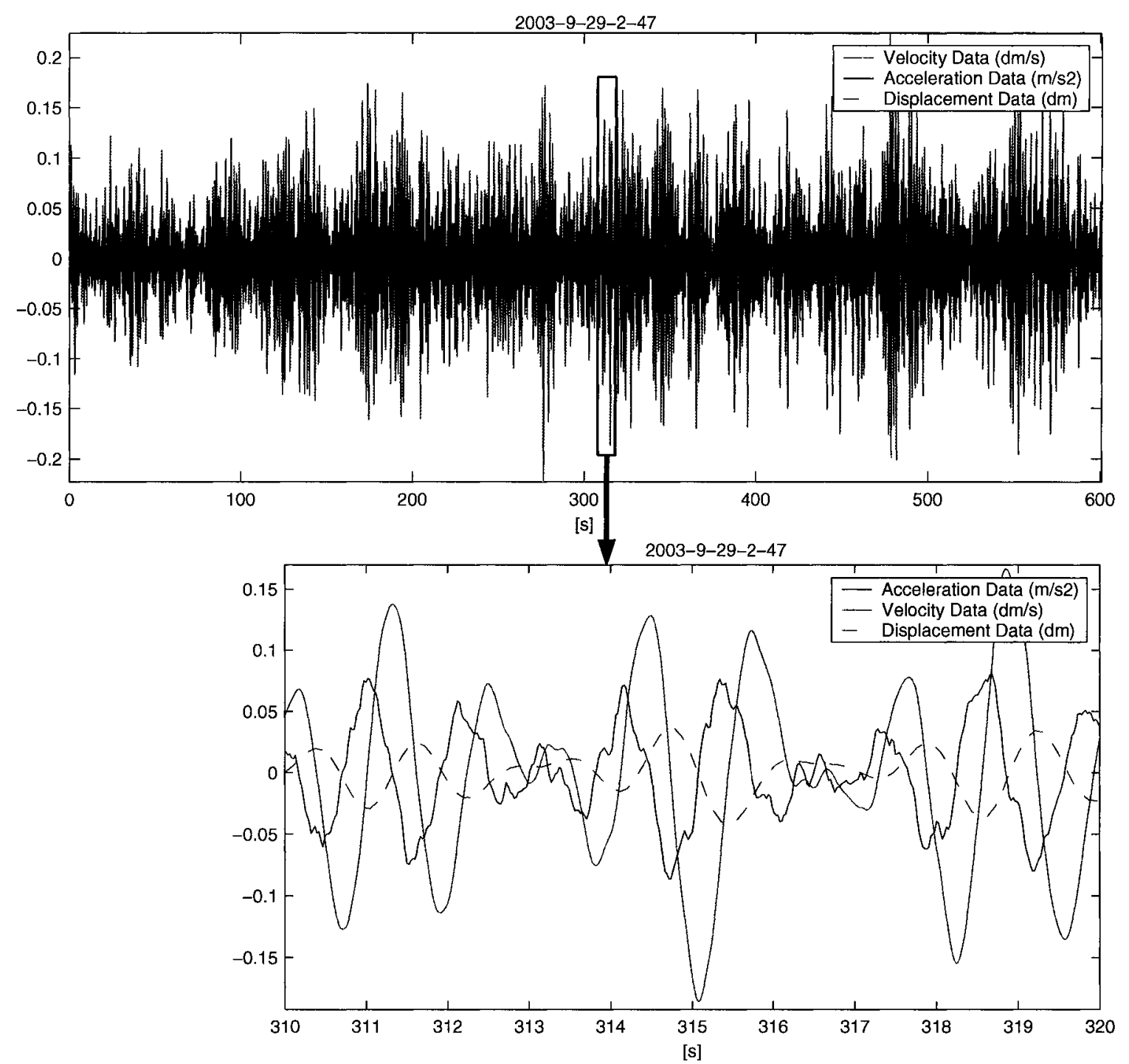

Figure 4.11. Integration of acceleration and velocity data to obtain displacement data. 


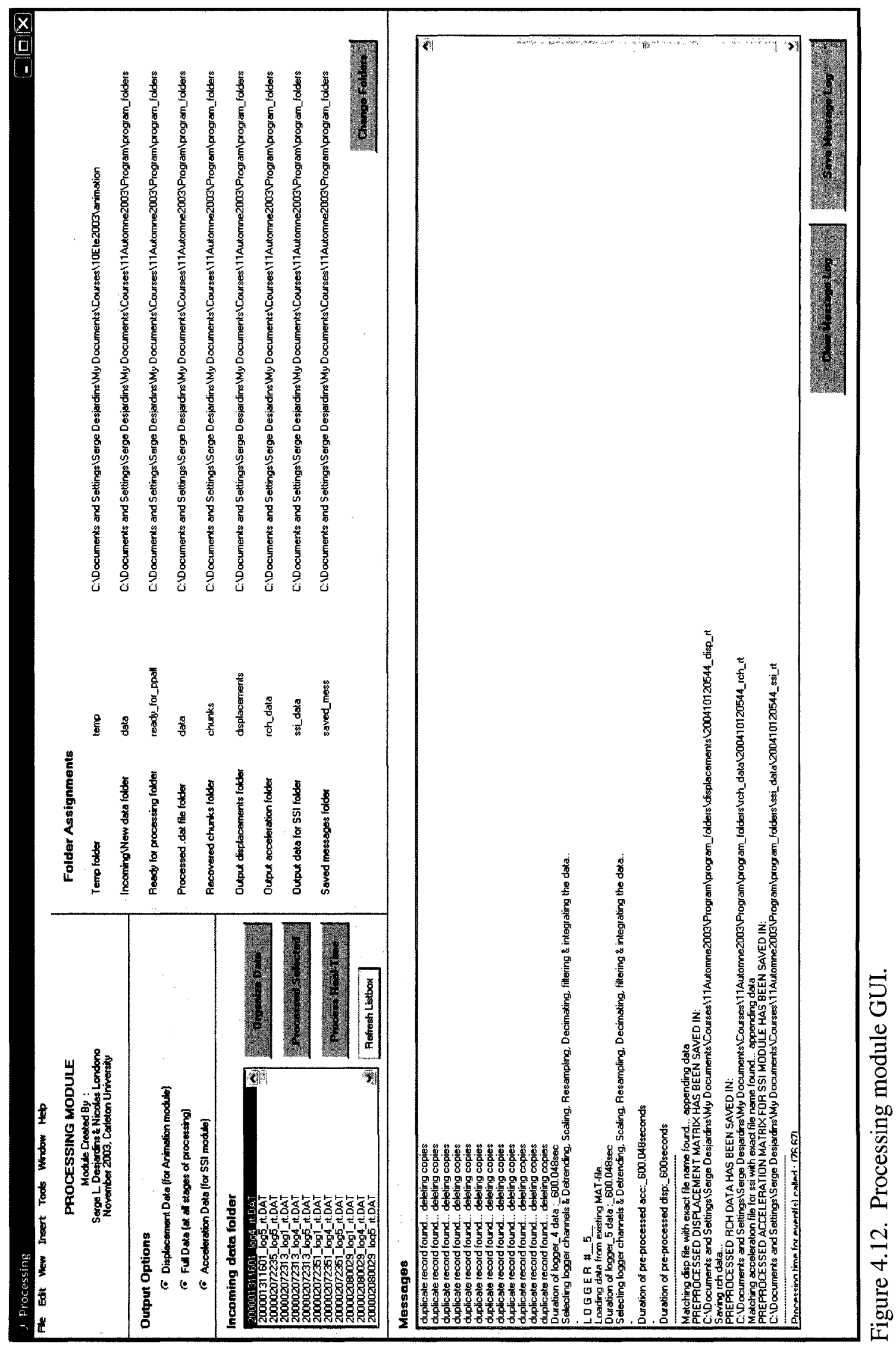




\section{Chapter 5}

\section{Operational Modal Analysis}

In design of structures, and particularly of large-scale complex structures, vibration behaviour is an important and potentially governing performance parameter that needs to be considered. With advances in construction technologies, new materials and new design techniques, larger and lighter structures, and thus more flexible structures, are constructed. The vibration behaviour and performance of these large-scale structures becomes an even more critical consideration in their design and safe operation. It is therefore important to have accurate models and tools to verify the design of complex structures, including the design assumptions adopted for their design and their safe operations in the field.

Sophisticated structural modeling techniques, such as complex finite element models are commonly used in structural design. Analytical structural models used for the design of a structure are typically developed based on design parameters and assumptions. The 
actual constructed structure may have some discrepancies from the theoretical models. The significance and impact of these discrepancies between the actual structure from its design specifications, and the differences between the actual field operating conditions of the structure and the design assumptions need to be evaluated and assessed. One approach for assessment of structural performance is by means of determining the dynamic properties of the structures. Frequencies, mode-shapes and damping ratios can be determined by using finite element models (FEM) of the structure. The FEM computer models can provide insight into the behaviour and performance of the Confederation Bridge subjected to a wide range of different load effects, such as earthquakes, ship impacts and wind. These insights and analytically determined structural behaviour have been used in the design of the Confederation Bridge. After its completion, it is therefore of interests and great importance to experimentally obtain the dynamic properties of the structure not only for design verification purposes but also for calibration of its computer structural models. For large scale complex structures like the Confederation Bridge, it is impractical or cost-prohibitive to carry out forced vibration measurements which has been the common practice and considered as the most reliable means for obtaining the dynamic characteristics of civil engineering structures. Consequently, ambient loading, without detailed knowledge of the sources, is relied upon as the input excitations of dynamic response measurements. Hence, in the operational modal analysis of the Confederation Bridge, output-only system identification techniques are required. The extracted dynamic properties of the Confederation Bridge are also used to establish baseline data sets needed for long-term performance and condition assessment of the structure in the future. The long-term behaviour and trends of the structure in terms of 
the structural vibration frequencies, mode-shapes and damping ratios are examined. Therefore, to ensure the highest possible accuracy and resolution of the data analysis results for establishing the baseline information for damage detection of the structure in the future, special system identification techniques are employed to extract structural dynamic properties of the bridge from the collected monitoring data and to analyze its data signatures.

Experimentally obtained modal properties can also provide critical insight into the behaviour and performance of the bridge during and after important events such as earthquakes or wind storms. Any shift or observed differences in the obtained frequencies may be considered as indications of potential structural problems.

In previous chapters, data capture, collection and transmission back to Carleton University from the bridge site, and processing have been discussed. In the following sections, the system identification techniques employed to analyze the response timehistory monitoring data to extract the dynamic modal properties of the Confederation Bridge are discussed. The analysis algorithms and tools developed for the Confederation Bridge monitoring project have been integrated in a modular platform with easy to use graphical user interfaces (GUI).

\subsection{Spectral Analysis}

The goal of spectral estimation is to describe the distribution over frequency of power contained in a signal, based on a finite number of sets of data. Estimation of power spectra is useful in a variety of applications, including the detection of signals buried in 
wide-band noise. The power spectrum of a stationary random process is mathematically related to the correlation sequence by the discrete-time Fourier transform. The Fourier transform, in essence, decomposes or separates the waveform of the signal into sinusoids of different frequency which sum to the original waveform.

\subsubsection{Welch's Power Spectral Density Estimate}

The power spectral density (PSD) function represents the power content of a signal in an infinitesimal frequency band. The unit of PSD is power (e.g., watts) per unit of frequency (watts/hertz). Integration of the PSD with respect to frequency yields units of watts, as expected for the average power. An improved estimator of the PSD by Welch (1967) is employed in the analysis of the Confederation Bridge monitoring data. The method consists of dividing the time series data into windowed overlapping segments, computing a modified periodogram of each segment, and then averaging the PSD estimates. The result is known as the Welch's PSD estimate.

The processed signal acquired from an individual accelerometer is divided into overlapping segments of size and overlap specified by the user. These segments are subjected to a windowing operation using a Hamming window (Figure 5.1) to prevent leakage in the Fast Fourier Transform (FFT) from time domain to frequency domain of the data. An $n$-point FFT is applied to the windowed data where $n$ is also user specified and is usually similar to the number of samples in the segment. The periodogram of each windowed segment is then computed to obtain the segment's power spectrum. The set of periodograms is averaged to form the spectrum of all segments which is then scaled with the sampling frequency to compute the power spectral density. Figures 5.2 and 5.3 
shows some sample power spectral densities of 10 minute signals captured during strong wind and traffic scenarios, respectively. The PSD of these figures are evaluated with a hamming window length of 8192 samples, a $50 \%$ segment overlap and $2^{13}$ points for FFT. The PSD of Figures 5.2 and 5.3 correspond to the time histories shown in Figures 4.1 and 4.2.

\subsubsection{Cross Power Spectrum (CPS)}

Another frequency domain analysis tool, which is implemented into the System Identification (SI) module of the integrated computer platform developed for monitoring of the Confederation Bridge, is the computation of Cross Power Spectrum (CPS) of the data signals. Initially a windowing operation is performed on the segmented data with a default segment overlap of $50 \%$. Fast Fourier transform of the segmented data is then computed which identifies or distinguishes the different frequency sinusoids and their respective magnitudes and phases (Brigham 1988). Figure 5.4 shows the magnitude and phase of the FFT of the original data segment shown in Figure 5.1(a) while Figure 5.5 shows magnitude and phase of FFT of the windowed segment shown in Figure 5.1(c) to better observe effects of windowing. In the algorithm, the CPS of all channels with respect to a subset of user specified reference channels is computed by multiplying their respective magnitudes of FFT. The channels mentioned here refer to acceleration measurement signals contained in the columns of the processed signal matrix described as an output in Chapter 4. Figure 5.6 shows the auto-power spectrum of the data segment shown in Figure 5.1(a). The auto-power is the cross-power of a signal with respect to itself. 


\subsection{Stochastic Subspace Identification Method}

Studies have shown that among the numerous system identification techniques proposed for civil engineering structural monitoring applications the stochastic subspace identification (SSI) method, also known as Covariance Block Hankel Matrix method (CBHM) (Bogunović Jakobsen 1995), is a robust output-only identification technique compared to other available methodologies (Peeters \& DeRoeck 1999, Peeters 2000). The SSI algorithm is similar to the Eigensystem Realization Algorithm with Data Correlations (ERA-DC) (Juang 1994) except that the ERA-DC method uses data correlations of the system impulse responses, which are estimated from both input and output measurements, instead of using data correlations of the output-only responses as in the SSI method.

The dynamic behaviour of physical systems, such as civil engineering structures, is traditionally modeled through discrete Finite Element (FE) approximation, which is represented by the following matrix equation of motion

$$
\mathbf{m u ̈}(t)+\mathbf{c u}(t)+\mathbf{k u}(t)=\mathbf{f}(t)=\mathbf{r p}(t)
$$

where $\mathbf{u}$ is the $n \times 1$ displacement vector of the $n$ degree of freedom (DOF) FE model, $\mathbf{m}$, $\mathbf{c}$, and $\mathbf{k}$ are the $n \times n$ mass, viscous damping, and stiffness matrices, respectively, $\mathbf{f}$ is an $n \times 1$ load vector, $\mathbf{r}$ is an $n \times m$ location coefficient that relates the locations of the $m$ inputs with the response degrees-of-freedom of the system model, and $\mathbf{p}$ is an $m \times 1$ vector describing the $m$ inputs or excitations. 
For system identification of discretely sampled responses, it is often more convenient to reformulate the FE model into a discrete-time state-space model form, which can be expressed as follows

$$
\begin{aligned}
& \mathbf{x}_{k+1}=\mathbf{A} \mathbf{x}_{k}+\mathbf{B} \mathbf{p}_{k} \\
& \mathbf{y}_{k}=\mathbf{C} \mathbf{x}_{k}+\mathbf{D} \mathbf{p}_{k}
\end{aligned}
$$

In this formulation, $\mathbf{x}_{k}$ is a $2 n \times 1$ state vector which describes the system displacement and velocity at the instant $k \Delta t$, where $\Delta t$ is the sampling interval, $\mathbf{p}_{k}$ is the sampled $m \times 1$ excitation vector, and $\mathbf{y}_{k}$ is an $l \times 1$ measured output vector. $\mathbf{A}$ is the discrete state matrix, $\mathbf{B}$ is the discrete input matrix, $\mathbf{C}$ is the discrete output matrix and $\mathbf{D}$ is the direct transmission matrix. Measured outputs (or responses) can be displacements, velocities or accelerations.

The state-space model formulation of Equation 5.2 is entirely deterministic. However, in civil engineering applications, especially in the context of continuous field monitoring, it is usually impractical or impossible to measure the input excitations, $\mathbf{p}_{k}$. Thus, the identification algorithms must be modified to rely on the measured outputs $\mathbf{y}_{k}$ only. As a result, in the SSI algorithm the input terms $\mathbf{B} \mathbf{p}_{k}$ and $\mathbf{D} \mathbf{p}_{k}$ are modeled as stochastic white noise terms. Thus, the discrete-time state-space model becomes a stochastic model expressed as follows

$$
\begin{aligned}
& \mathbf{x}_{k+1}=\mathbf{A} \mathbf{x}_{k}+\mathbf{w}_{k} \\
& \mathbf{y}_{k}=\mathbf{C} \mathbf{x}_{k}+\mathbf{v}_{k}
\end{aligned}
$$

Both $\mathbf{w}_{k}$ and $\mathbf{v}_{k}$ are assumed to be white, zero-mean stochastic processes, independent of the state vector. Based on these assumptions, from the stochastic state-space model formulation of Equation 5.3, it can be shown that the correlations of the outputs $\mathbf{R}_{i}$ can be factorized into a triplet containing the state matrices (Peeters 1999, 2000) 


$$
\mathbf{R}_{i}=\mathbf{C A}^{i-1} \mathbf{G}
$$

where the output correlation matrices $\mathbf{R}_{i}$ are defined as $\mathbf{R}_{i}=\mathrm{E}\left[\mathbf{y}_{k+i} \mathbf{y}_{k}^{T}\right]$, and $\mathrm{E}$ denotes the expectation operator. The subscript $i$ indicates a time lag of $i \Delta t$, and $\mathbf{G}=\mathrm{E}\left[\mathbf{x}_{k+1} \mathbf{y}_{k}^{T}\right]$ is a "next-state-output" correlation matrix. Equation 5.4 represents the basis of the correlation driven SSI algorithm. It indicates that suitable de-composition of the correlations can yield the state-space matrices, which in turn contain the structural parameters of mass, stiffness and damping. Data correlations also offer the advantages of eliminating uncorrelated noise and compressing the data while preserving the modal information.

Estimation of the correlations from the data is performed according to the following expression

$$
\hat{\mathbf{R}}_{i}=\frac{1}{N} \sum_{k=0}^{N-1} \mathbf{y}_{k+i} \mathbf{y}_{k}^{T}
$$

where $\mathbf{y}_{k}$ is a column vector of the measured responses at the sampling instant $k \Delta t$ and $N$ is the number of samples. In operational modal analysis applications, typically many sensors are used to measure the response of the structural system in order to obtain the mode-shapes with a reasonable spatial resolution. As a result, there is usually some redundancy in the data because all sensors carry essentially the same information regarding the frequency and damping ratios, except if they are located at modal nodes. Taking advantage of this observation the amount of computations can be substantially reduced by computing data correlations with respect to just a small subset of reference sensor channels. Data correlations at a sequence of different time lags are assembled into a block Toeplitz matrix as follows 


$$
\mathbf{T}_{1 i i}^{r e f}=\left[\begin{array}{cccc}
\mathbf{R}_{i}^{r e f} & \mathbf{R}_{i-1}^{r e f} & \ldots & \mathbf{R}_{1}^{r e f} \\
\mathbf{R}_{i+1}^{r e f} & \mathbf{R}_{i}^{\text {ref }} & \ldots & \mathbf{R}_{2}^{\text {ref }} \\
\ldots & \ldots & \ldots & \ldots \\
\mathbf{R}_{2 i-1}^{r e f} & \mathbf{R}_{2 i-2}^{r e f} & \ldots & \mathbf{R}_{i}^{r e f}
\end{array}\right]
$$

where $\mathbf{R}_{i}^{r e f}=\mathrm{E}\left[\mathbf{y}_{k+i}\left(\mathbf{y}_{k}^{r e f}\right)^{T}\right], \mathbf{y}_{k}^{r e f}$ is the $r \times 1$ vector of sampled reference responses at time instant $k, r$ is the number of reference channels, and $i$ is a parameter that defines the number of time lags of the data correlations to be used for system identification. This parameter must satisfy the relation $r \times i>2 n$, where $2 n$ is the order of the state-space model. The factorization property of Equation 5.4 allows the following factorization of the block Toeplitz matrix

$$
\mathbf{T}_{1 \mid i}^{r e f}=\left[\begin{array}{c}
\mathbf{C} \\
\mathbf{C A} \\
\ldots \\
\mathbf{C A}^{i-1}
\end{array}\right]\left[\begin{array}{llll}
\mathbf{A}^{i-1} \mathbf{G}^{\text {ref }} & \ldots & \mathbf{A} \mathbf{G}^{\text {ref }} & \mathbf{G}^{\text {ref }}
\end{array}\right]=\mathbf{O}_{i} \boldsymbol{\Gamma}_{i}^{\text {ref }}
$$

where $\mathbf{O}_{i}$ and $\boldsymbol{\Gamma}_{i}^{r e f}$ are rank $2 n$ matrices known as the extended observability and reversed extended stochastic controllability matrices, respectively. The Singular Value Decomposition (SVD) technique is then used to reduce the block Toeplitz matrix into suitable factors as follows

$$
\mathbf{T}_{1 \mid i}^{\text {ref }}=\mathbf{U S V}^{T}=\left[\begin{array}{ll}
\mathbf{U}_{1} & \mathbf{U}_{2}
\end{array}\right]\left[\begin{array}{cc}
\mathbf{S}_{1} & \mathbf{0} \\
\mathbf{0} & \mathbf{0}
\end{array}\right]\left[\begin{array}{c}
\mathbf{V}_{1}^{T} \\
\mathbf{V}_{2}^{T}
\end{array}\right]=\mathbf{U}_{1} \mathbf{S}_{1} \mathbf{V}_{1}^{T}
$$

where $\mathbf{S}_{1}$ is a diagonal matrix containing singular values in descending order. Using Equations 5.7 and 5.8, the following so-called internally balanced realization may be obtained

$$
\mathbf{O}_{i}=\mathbf{U}_{1} \mathbf{S}_{1}^{1 / 2}
$$




$$
\boldsymbol{\Gamma}_{i}^{r e f}=\mathbf{S}_{1}^{1 / 2} \mathbf{V}_{1}^{T}
$$

The state-space matrix $\mathbf{A}$, which contains the mode-shapes and eigenvalues, can be extracted from the extended observability matrix as follows

$$
\mathbf{A}=\mathbf{O}_{i}(1: l(i-1),:)^{+} \mathbf{O}_{i}(l+1: l i,:)
$$

where $l$ is the number of output channels, $i$ is the number of block rows of the block Toeplitz matrix, $\mathbf{O}_{i}(1: l(i-1),:)$ and $\mathbf{O}_{i}(l+1: l i,:)$ denote rows 1 to $l(i-1)$ and $(l+1)$ to $l_{i}$ of the extended observability matrix respectively, and "*+" denotes the Moore-Penrose pseudo-inverse. The eigenvalue decomposition of the discrete-time state matrix $\mathbf{A}$ yields

$$
\mathbf{A}=\boldsymbol{\Psi} \Lambda \boldsymbol{\Psi}^{-1}
$$

where $\Psi$ is a complex eigenvector matrix and $\Lambda$ is a diagonal matrix containing the discrete-time eigenvalues $\mu_{\mathrm{j}}$, which are directly related to the system "poles" $\lambda_{j}$ or eigenvalues of the original second order system described by Equation 5.1. The system poles contain the modal frequencies $\omega_{j}$ and damping ratios $\xi_{j}$

$$
\lambda_{j}=\frac{\log _{e}\left(\mu_{j}\right)}{\Delta t}=-\xi_{j} \omega_{j}+i \sqrt{1-\xi_{j}^{2}} \omega_{j}
$$

The observed mode-shapes at sensor locations are computed as follows

$$
\mathbf{V}=\mathbf{C} \boldsymbol{\Psi}
$$

where $\mathbf{C}$ can be obtained directly as the first $l$ rows of $\mathbf{O}_{i}$, as shown in Equation 5.7.

In practice, the state-space order of the structural system $(2 n)$ is unknown. Theoretically, the order can be determined by looking at the number of non-zero singular values in matrix $\mathbf{S}$ of Equation 5.8. However, when analyzing the data from real, complex structures such as the Confederation Bridge, the model order is typically masked by the 
noise in the data and the matrix $\mathbf{S}$ appears to have all non-zero singular values. The noise in the data may arise from a combination of the following approximations:

- Modeling approximations;

- Measurement inaccuracies;

- The use of finite data sets to compute estimates of the correlations;

- Non-stationarity and non-linearity behaviour in the data and structure.

The processing and analysis of the monitoring data from civil engineering structures contaminated or corrupted by noises or other uncertainties are especially challenging to the development of robust and reliable system identification techniques and algorithms for civil engineering applications. To overcome the problem of the unknown and hidden system order, a technique known as stabilization can be employed. In this technique, a set of models of different $\operatorname{order}\left(\right.$ half-order $n$ from $n_{\min }=1$ to $n=n_{\max }$ ) are identified by including a different number of singular values and vectors from the SVD in the computation of $\mathbf{O}_{i}$ and $\Gamma_{i}$ (Equations 5.9 and 5.10). The solutions from the models of different order are presented in a stabilization diagram where the distinction between 'true' or stable system solutions and 'spurious' solutions caused by the noise becomes apparent. A typical stabilization diagram is shown in Figure 5.7. A more rigorous and indepth treatment of the SSI algorithm can be found in the references (Peeters \& DeRoeck 1999, Peeters 2000). 


\subsection{Analysis Modules with Graphical User Interface}

The processed signal matrix obtained from the processing engine described in Chapter 4 can be subjected to Welch's PSD estimate algorithm in a specialized module which permits visualization and comparison of accelerometer signals time histories and their corresponding PSD estimates in the frequency domain. The spectral analysis engine integrated in the visualization module is a very useful tool to obtain rapid insight into the behaviour of the monitoring data in terms of power content and signatures of a given measurement channel. The natural frequencies of the system can be identified from the spectral analysis plots by peak picking methods. Figure 5.8 shows the data display module. Welch's PSD estimate parameters that are user specified include:

- Hamming window length;

- Segment overlap;

- Number of points for FFT;

- Frequency range of interest.

The System Identification module shown in Figure 5.9 integrates two main algorithms: the Cross-Power Spectra computation and the Stochastic Subspace Identification algorithm described above. A typical analysis session begins by importing the acceleration matrix generated by the processing module. There is also the option of importing an existing analysis session which has been saved previously. The user specified input options include:

- Channels from the acceleration matrix to include in the analysis;

- Subset of channels to be used as references;

- Maximum system model order number $n$; 
- Number of time lags, $i$, of the data correlations;

- Tolerances for solution pole stability;

- The number of points for FFT calculations;

- The CPS reference pairs.

The CPS between data channels are used in the stabilization diagram algorithm to aid user-selection of the stable poles. Cross-power of any single data channel with respect to any single user-selected reference channel can be plotted. In the system identification module there is an option for evaluating and plotting the sum of normalized cross-powers of all data channels with respect to the reference channels. The procedures of the CPS algorithm for background spectrum plot in the stabilization diagram of the SSI module are summarized as follows:

- A matrix $\mathbf{Y}$ of $L$ rows and $N_{o}$ columns is constructed where $L$ corresponds to the number of channels chosen for analysis and $N_{o}$ is the number of samples in the response signal data file.

- Each row is divided into segments of size determined from the user-specified number of points for FFT. For CPS computations in the system identification module, the segment size is made equal to the number of points for FFT and segment overlap is set to a default value of $50 \%$;

- The segments of each row are windowed by a Hamming window of length equal to the number of points selected for FFT;

- FFT of the data segments is computed;

- A sub-matrix containing the selected reference channels is identified from within $\mathbf{Y}$; 
- The Cross-Power Spectrum of each response data channel with respect to each of the selected reference data channel is computed for each segment and then stored in a 4 dimensional matrix where the fourth dimension represents the data segments;

- The calculated CPS is then averaged over all segments $\left(4^{\text {th }}\right.$ dimension). The results are then stored in a 3 dimensional matrix;

- The algorithm identifies the user selected channel pairs and then retrieves corresponding spectral information from the 3D matrix;

- The spectral information of each selected channel pair is then plotted in the background of the stabilization diagram;

- As an option of the module, a sum of each normalized spectrum (normalized with respect to maximum) contained in the 3D matrix can be calculated and plotted.

The SSI method described earlier has been implemented in its own module, the system identification module, and integrated to work with other modules of the computer platform. The main components of the SSI algorithm is shown in Appendix D and the relevent steps are briefly summarized here:

- The first operation consists in constructing the block Toeplitz matrix of Equation 5.6 from the $L \times N_{o}$ matrix of the chosen channels $\mathbf{Y}$, where $L$ represents the number of chosen channels and $N_{o}$ represents the number of samples. A "future" matrix $\left(\mathbf{Y}_{f}\right)$ and a "past" matrix $\left(\mathbf{Y}_{p}\right)$ are constructed from $\mathbf{Y}$ with a user-specified time-lag, $i$. For computation efficiency a $\mathbf{Y}_{\text {pref }}$ matrix is constructed from the $\mathbf{Y}_{p}$ matrix using the user-specified reference channels. Figure 5.10 shows how these matrices are constructed from $\mathbf{Y}$. The block Toeplitz matrix is obtained by multiplying $\mathbf{Y}_{f}$ by the 
transpose of $\mathbf{Y}_{\text {pref }}$ and dividing by $N$. This gives a large $\left(L^{*} i \times r * i\right)$ Toeplitz matrix, where $r$ is the number of reference channels.

- The singular value decomposition of the Toeplitz matrix is then performed by a Matlab function to obtain the $\mathbf{S}, \mathbf{U}$, and $\mathbf{V}$ matrices.

- Sub-matrices $\left(\mathbf{S}_{1}, \mathbf{U}_{1}\right.$, and $\left.\mathbf{V}_{1}\right)$, whose size depends on the model order at which a solution is being computed, are extracted from $\mathbf{S}, \mathbf{U}$, and $\mathbf{V}$.

- The observability $\left(\mathbf{O}_{i}\right)$ matrix is obtained at each model order (from 1 to userspecified $n_{\max } / 2$ ) by multiplying $\mathbf{U}_{1}$ with the square root of $\mathbf{S}_{1}$ (Equation 5.9).

- The stochastic controllability matrix $\left(\Gamma_{i}\right)$ is obtained at each model order by multiplying the square root of $S_{1}$ by the transverse of $\mathbf{V}_{1}$ (Equation 5.10).

- The state space matrix $\mathbf{A}$ is extracted for each model order from the observability matrix as per Equation 5.11 where $l=L$.

- The discrete output matrix $\mathbf{C}$ is also extracted for each model order from $\mathbf{O}_{i}$ by taking the first $L$ rows.

- The eigenvalues and eigenvectors are obtained from the eigenvalue decomposition of the state space matrix $\mathbf{A}$ at each model order.

- The frequency and damping ratio solution at each model order is calculated from the eigenvalues that have a positive imaginary component.

- The mode-shapes are obtained by multiplying the discrete output matrix $\mathbf{C}$ and the eigenvector matrix, where the columns represent the eigenvectors.

- All solutions at each model order are stored in a Matlab structure.

The final step of the algorithm is the plotting of the solution poles in the stabilization diagram where the user then picks from a series of computed poles the stable solutions. 
The SSI algorithm identifies a series of poles at different model orders; a stable pole is one consistently identified through the model orders. Solutions at a given model order are compared to those of the previous model order to determine their stability based on user-specified tolerances on frequencies, damping ratios and mode-shapes. The comparison of the mode-shapes is evaluated by consideration of the modal assurance criterion (MAC) (Londoño 2003b, Peeters 2000).

$$
\operatorname{MAC}\left(\boldsymbol{\Psi}_{i}, \boldsymbol{\Psi}_{j}\right)=\frac{\left|\boldsymbol{\psi}_{i}^{*} \boldsymbol{\psi}_{j}\right|^{2}}{\left(\boldsymbol{\Psi}_{i}^{*} \boldsymbol{\psi}_{i}\right)\left(\boldsymbol{\psi}_{j}^{*} \boldsymbol{\psi}_{j}\right)}
$$

where $\psi_{i}$ and $\psi_{j}$ are two different mode-shape column vectors, the symbol " * " indicates complex conjugate transpose. An MAC value of 1 indicates perfect correlation, while a value of 0 indicates no correlation. Number characters from 0 to 4 are plotted in the diagram and represent the different states of the solution poles:

\section{New Pole;}

1. Frequency only is stable;

2. Frequency and damping are stable;

3. Frequency and mode-shape are stable;

4. Stable pole (Frequency, mode-shape and damping ratio are all stable within the specified tolerances).

The user chooses system solutions by clicking the numbered characters on the diagram plot. The background CPS plots help to correlate the stable poles with the spectral peaks. An algorithm identifies the chosen pole and updates the "identification results" window which provides information on the picked solution. Once user has identified all solutions a parametric solution can be computed from the chosen solutions and plotted above the 
existing CPS plot(s) to validate the chosen solutions. The mode-shapes from the chosen solutions can also be visualized and validated by comparison with other mode-shapes in the animation module detailed in Chapter 6 of this thesis. Feedback is given to the user through detailed comprehensive messages. An RMS calculation for the vertical and transverse signals is automatically performed and result is displayed on the GUI. The sessions can be saved and re-opened for further analysis. Once saved, a session information structure of matrices is created and contains the modal properties which can be used for subsequent statistical information or implementation into a condition assessment algorithm. 


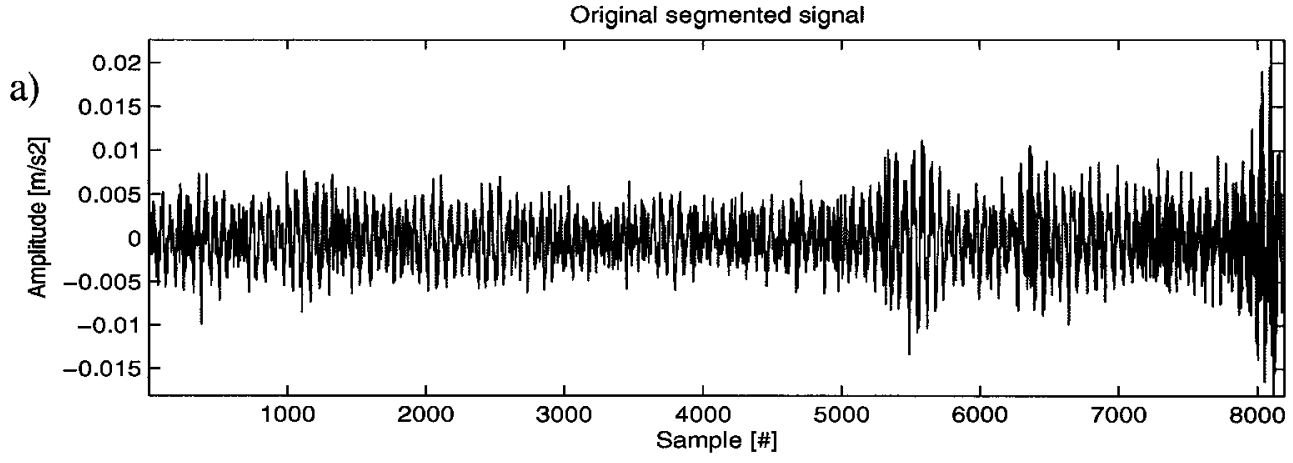

b)
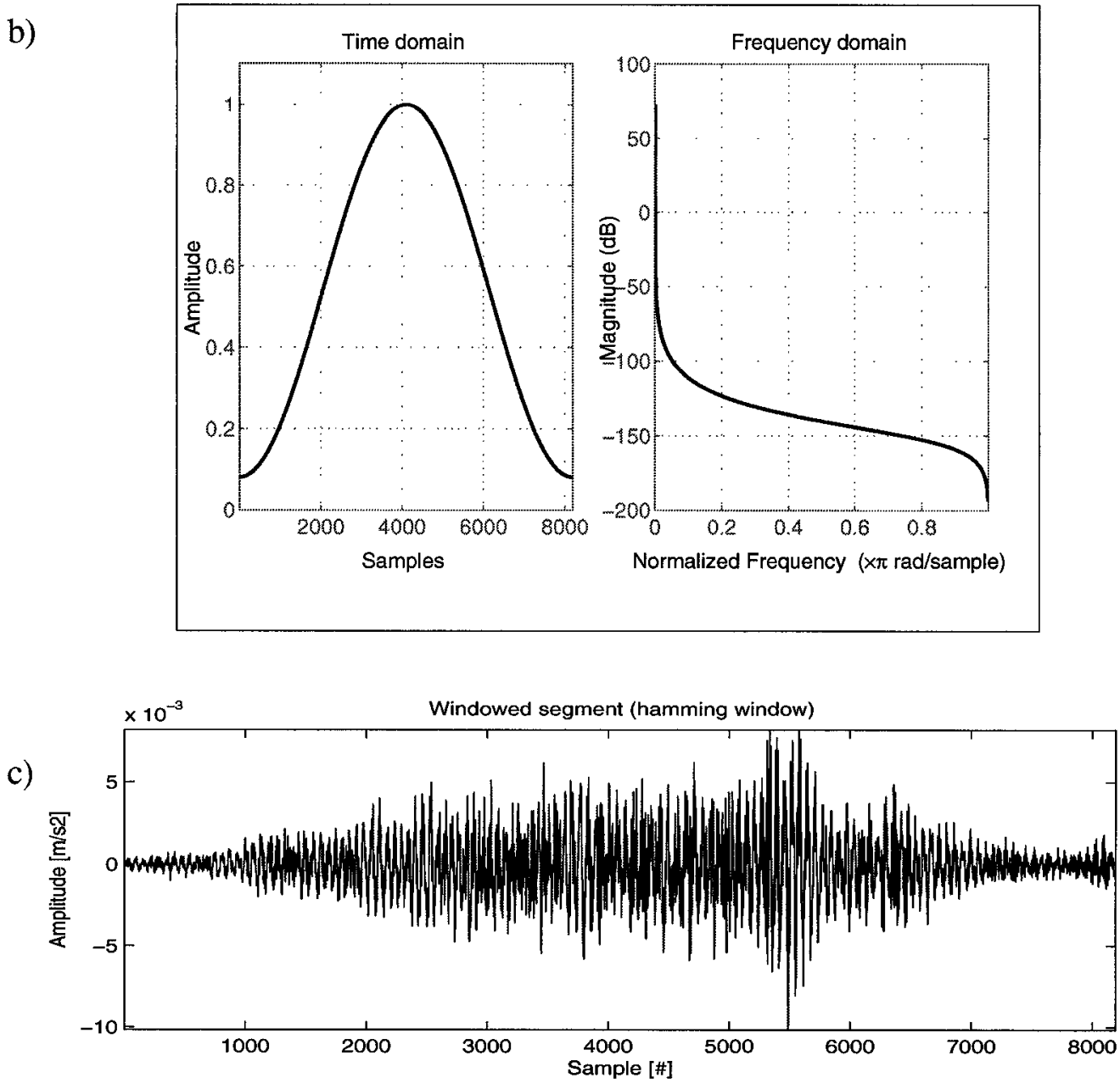

Figure 5.1. Example of a windowed segment of data subjected to a hamming window of length equal to 8192 samples. a) Original data segment of 8192 samples recorded by a Confederation Bridge accelerometer. b) Hamming window characteristics. c) Windowed data segment. 

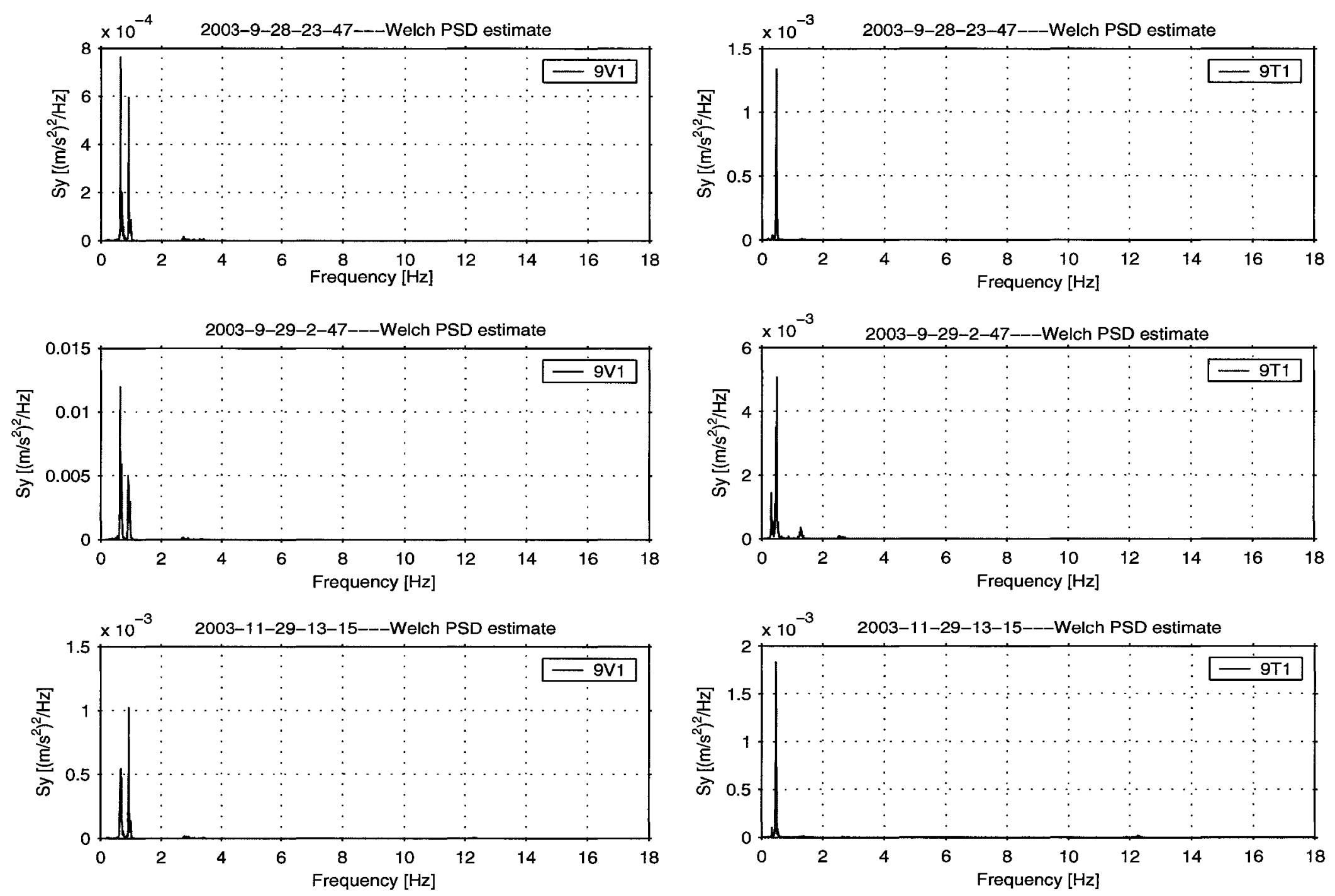

Figure 5.2. Power spectral density estimates of signals captured under strong wind. (Time histories are shown in Figure 4.1) 

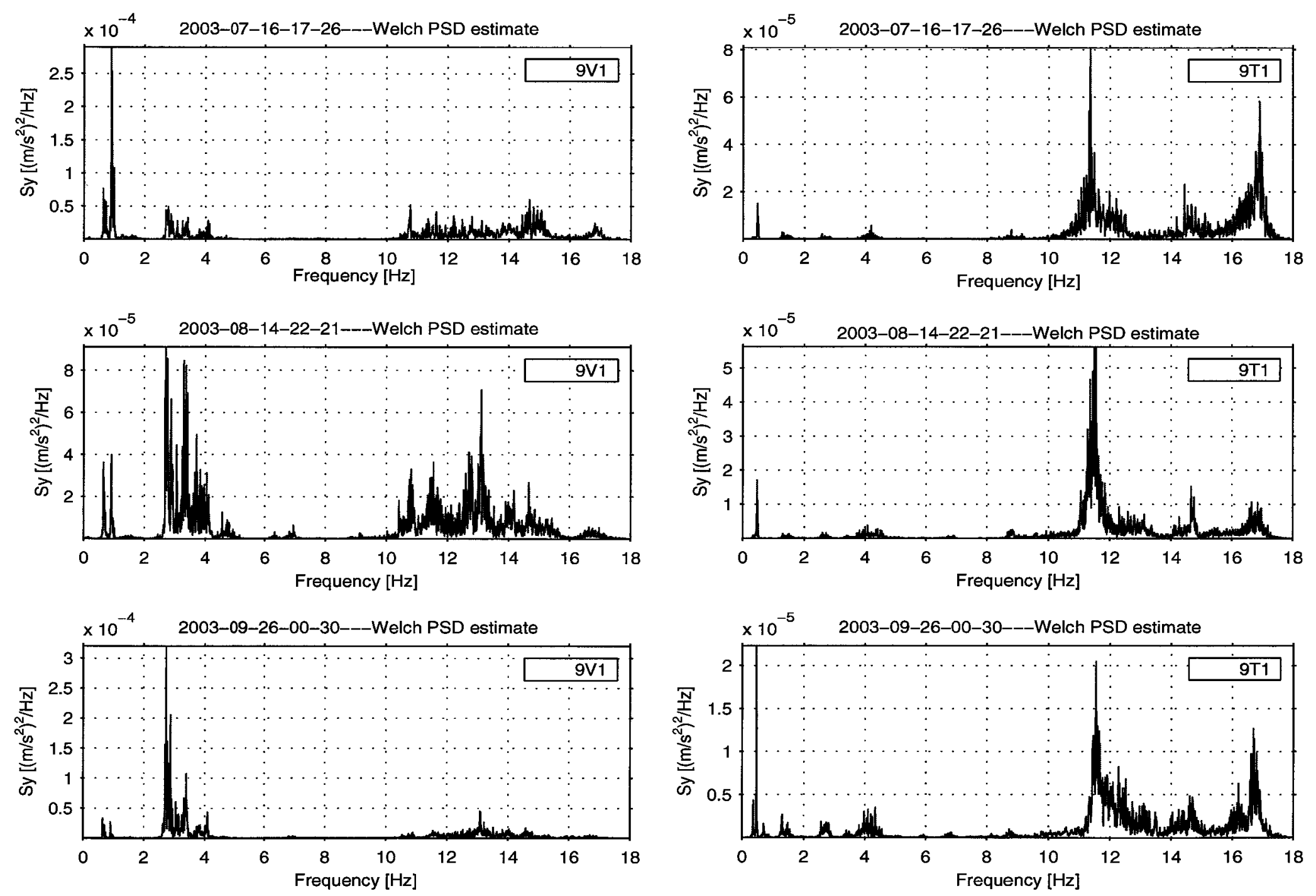

Figure 5.3. Power spectral density estimates of signals captured during traffic. (Time histories are shown in Figure 4.2) 

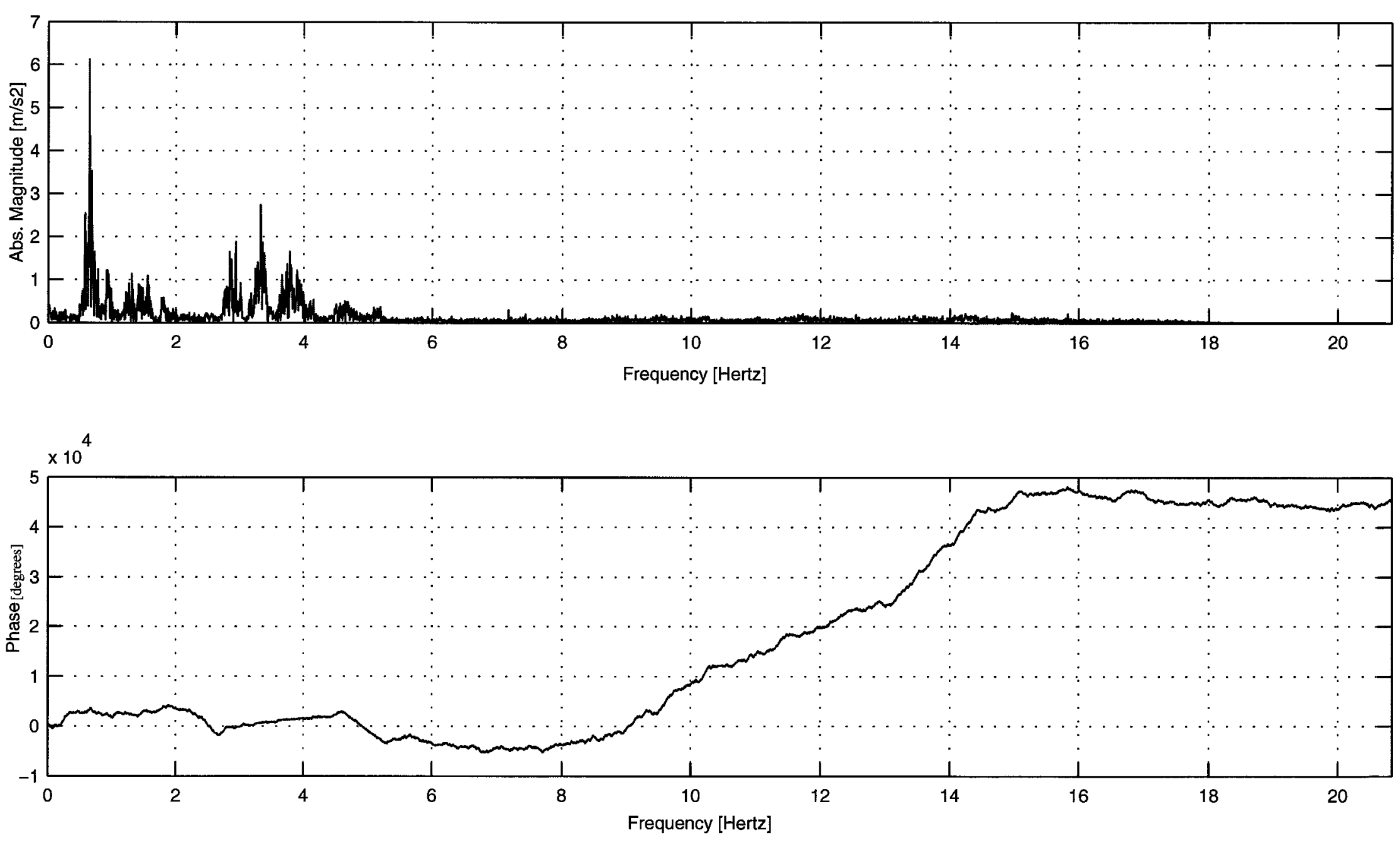

Figure 5.4. Typical FFT of time history signal shown in Figure 5.1(a). 

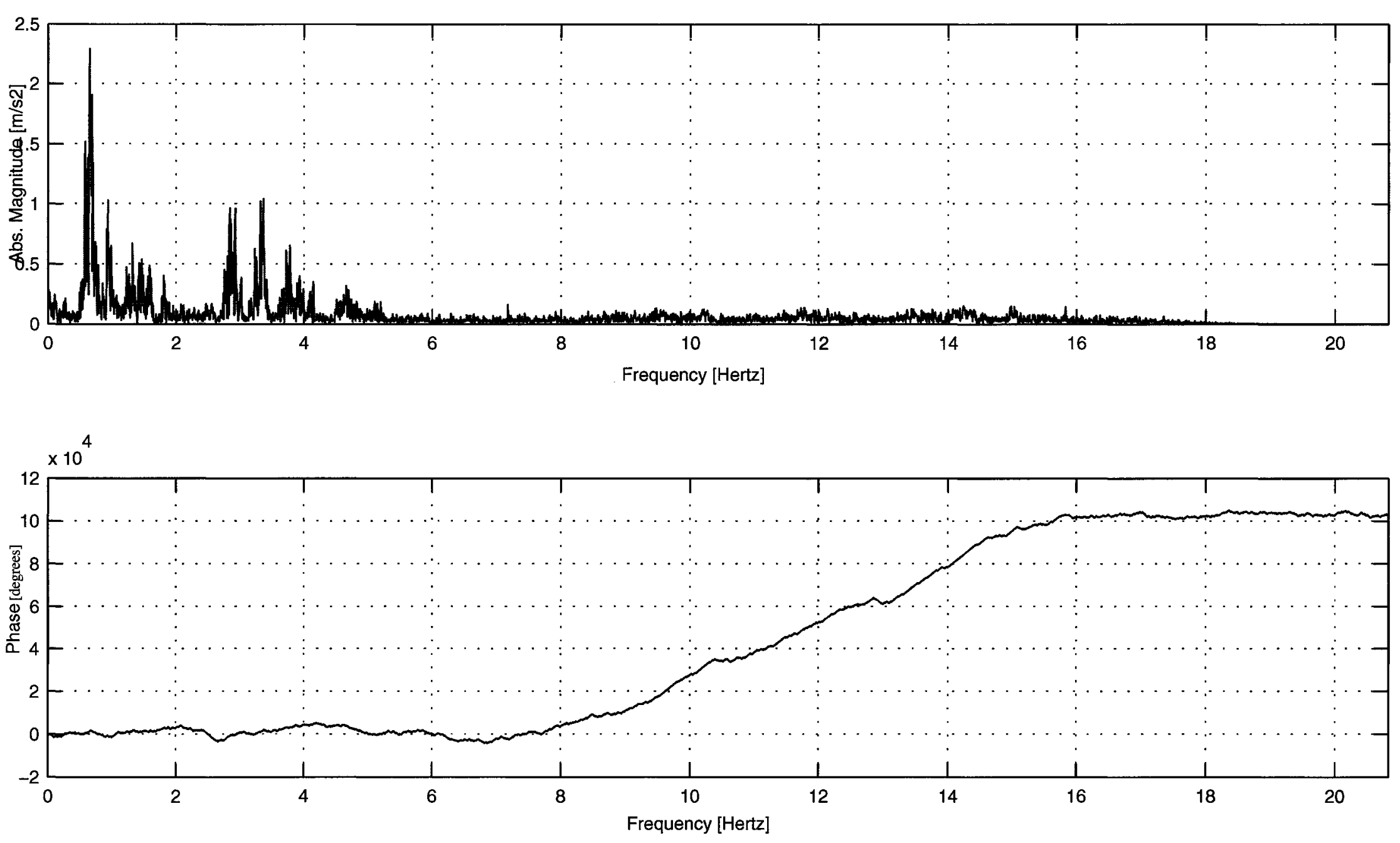

Figure 5.5. Typical FFT of windowed time history signal shown in Figure 5.1(c). 


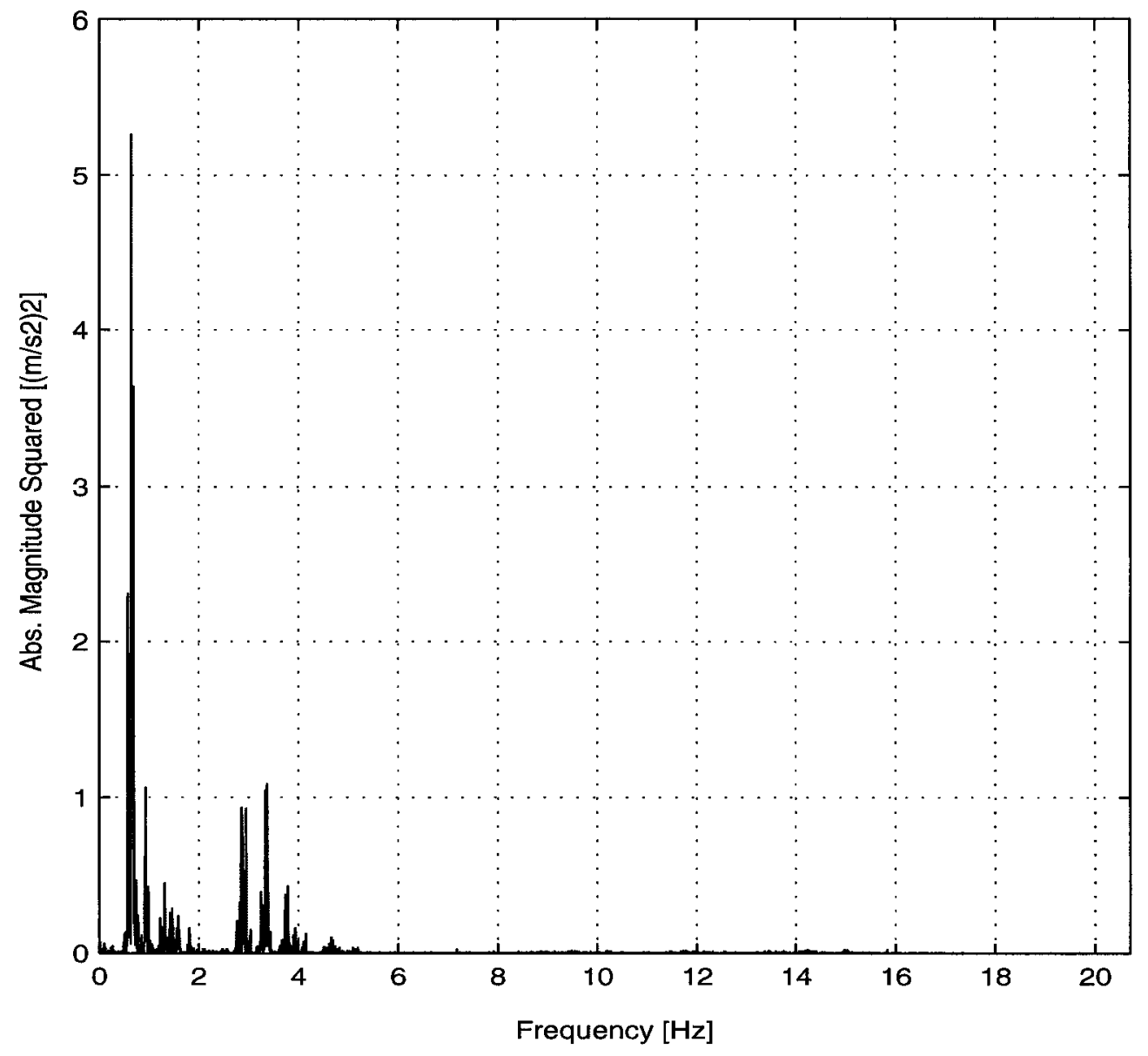

Figure 5.6. Typical auto-power spectrum of a signal. Time history of spectrum shown here is found in Figure 5.1(a) 


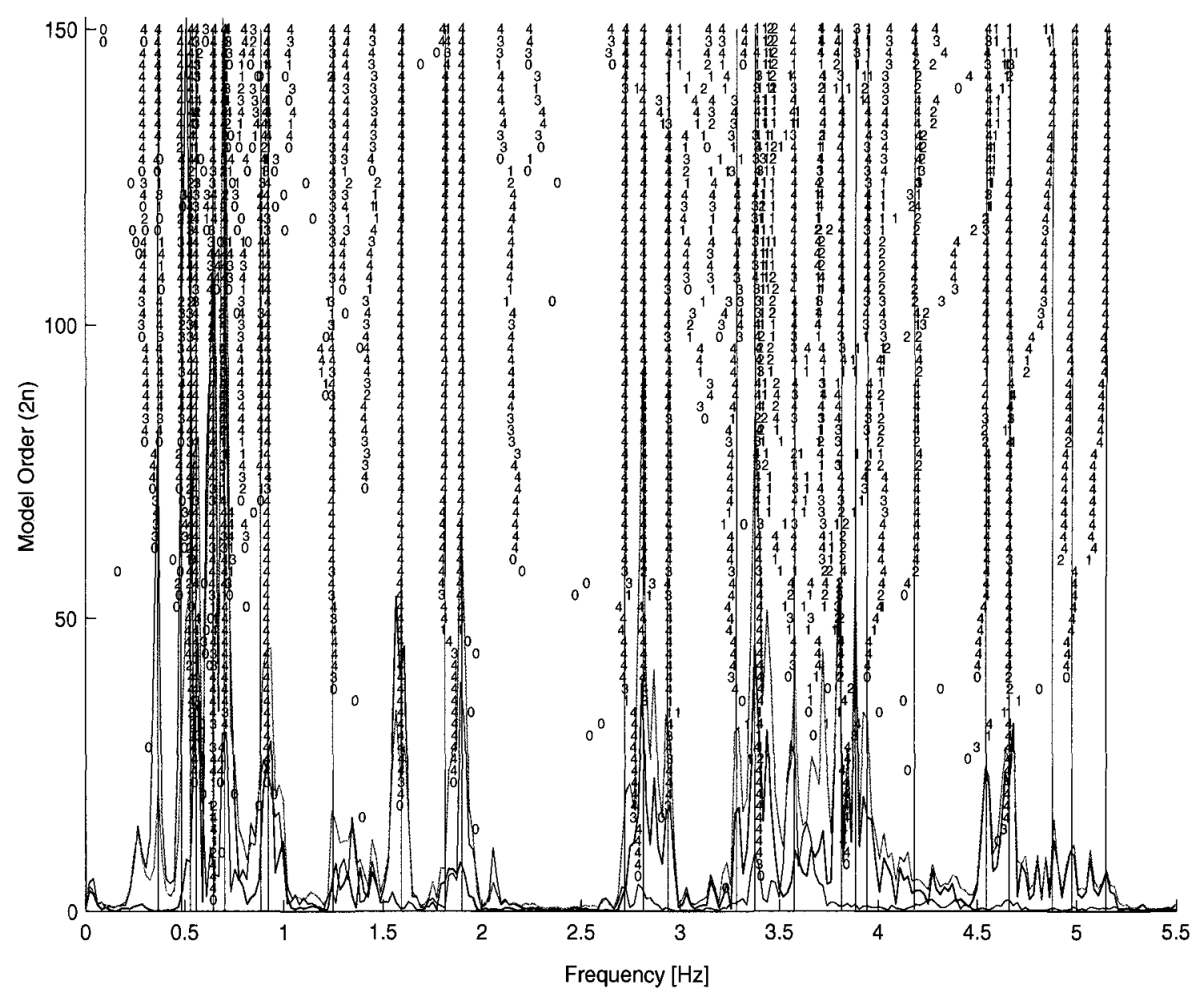

Figure 5.7. Typical stabilization plot showing stability of poles. 


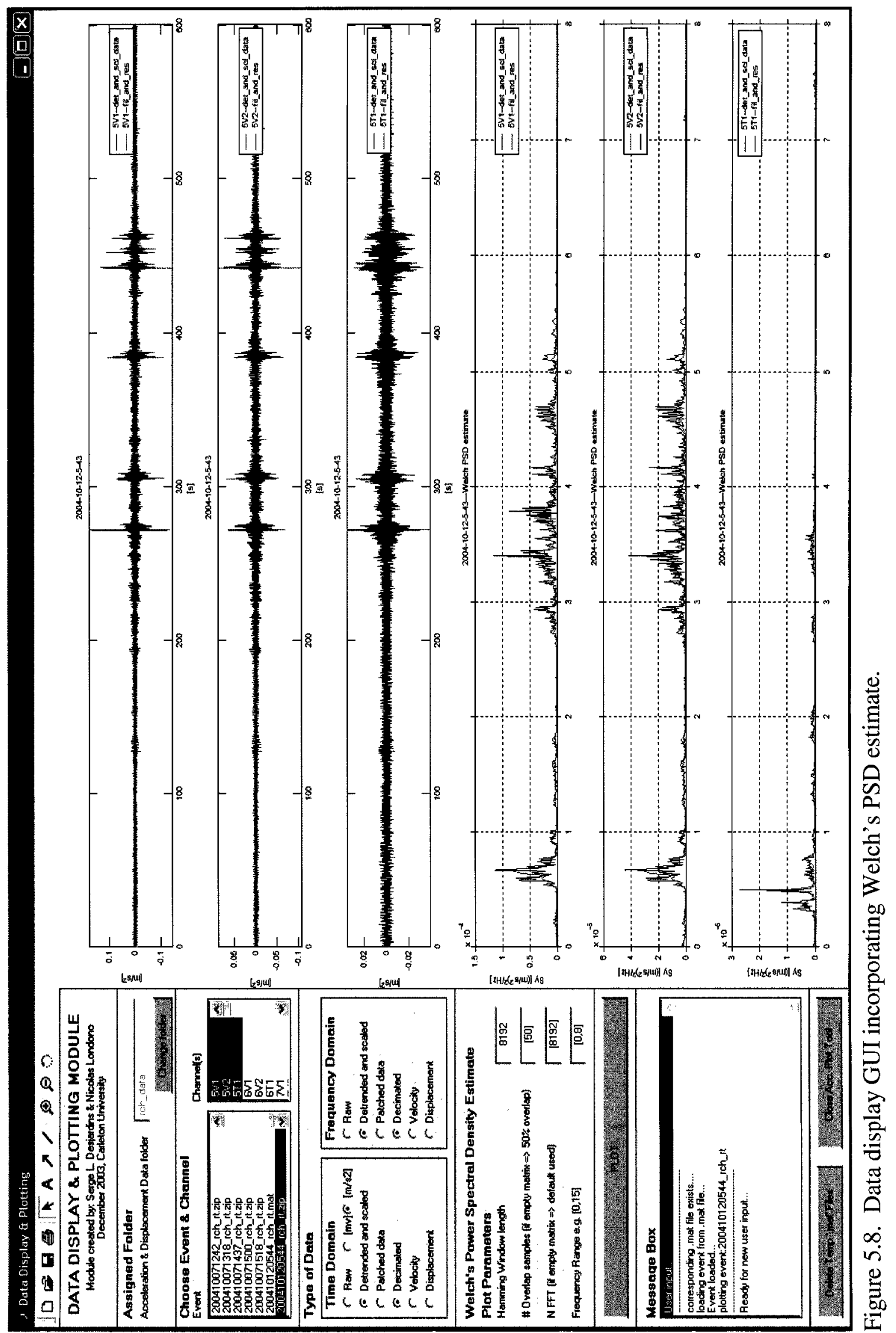




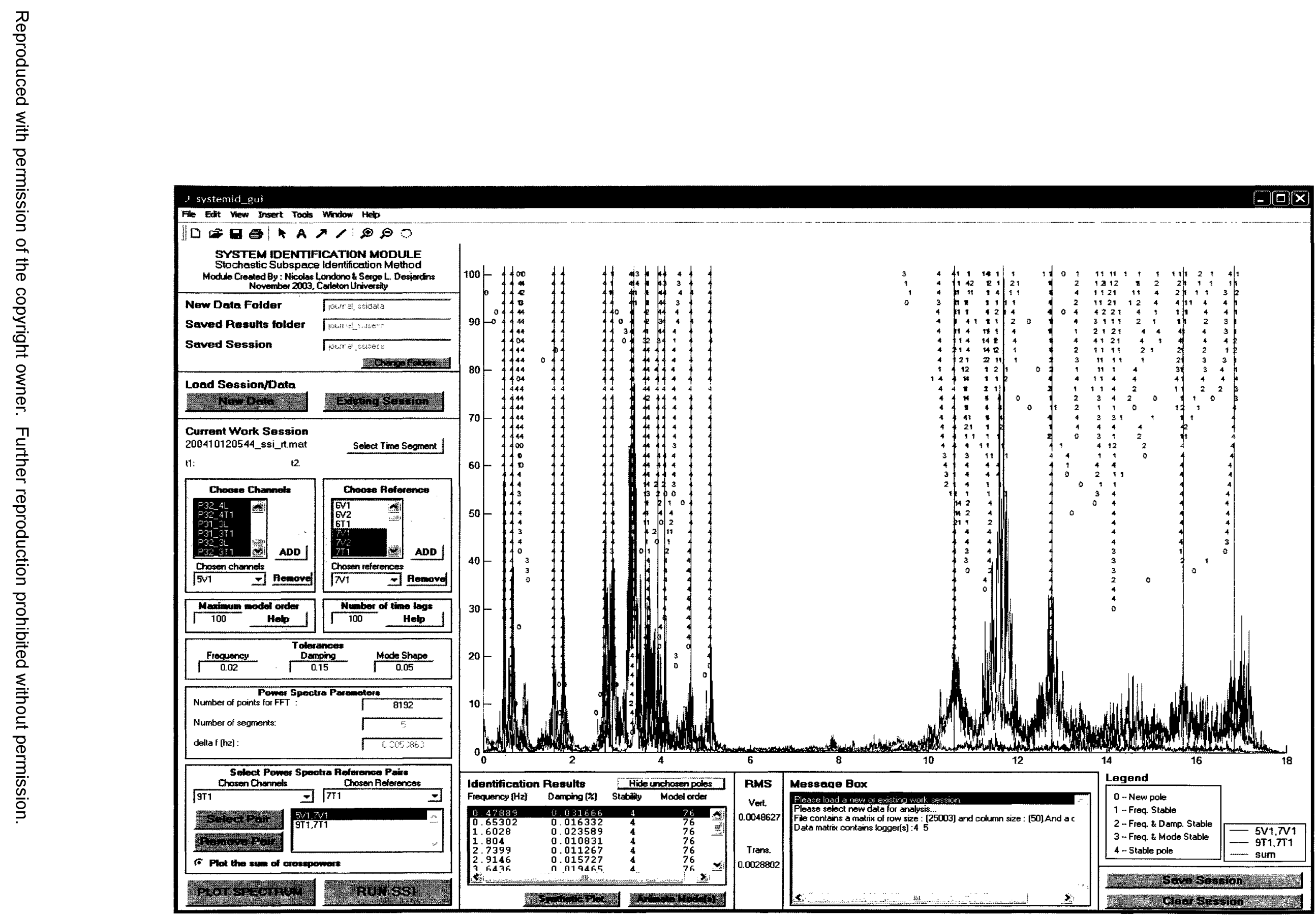

Figure 5.9. System Identification GUI incorporating the SSI algorithm with stabilization diagram and background CPS computations. 

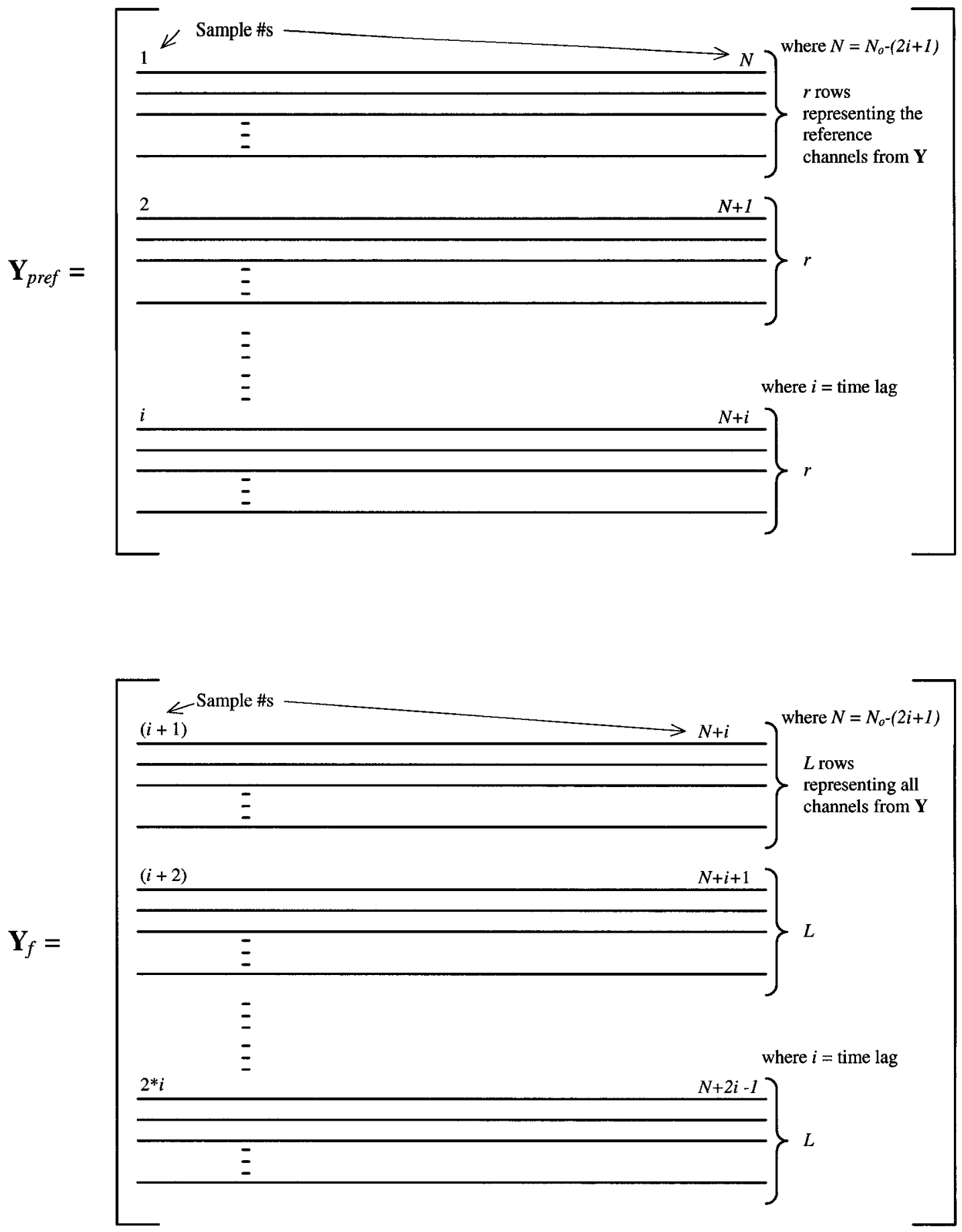

Figure 5.10. Assembling of the $\mathbf{Y}_{\text {pref }}$ and $\mathbf{Y}_{f}$ matrices for the block Toeplitz matrix. 


\section{Chapter 6}

\section{Real-Time Visualization of Vibration Monitoring Data}

The modules developed for data visualization help to facilitate the extraction of engineering information from the large data sets of monitoring data in a timely manner. The data files of acceleration time history measurements are processed and transformed into frequency domain to analyze characteristics of the data signatures. Using the visualization modules, response time histories and extracted modes obtained from system identification analysis of the monitoring data can be animated in 3D for ease of visualization and thus understanding the behaviour of the structure. With easy to use, flexible and effective visualization tools, vibration behaviour of complex structures can be easier to understand and more detailed information can be extracted from the monitoring data about the state or condition of the structure. Figure 5.8 and 6.1 show 
screen captures of the data display and animation visualization modules. The design and operations of the 3D animation visualization and data display modules are described in this chapter.

The processing and animation modules are designed to have the capability to operate in real-time mode. A tool has been developed to automatically detect incoming data from the bridge and direct it to the proper module for data management and processing steps. The processing results can then be animated or visualized in real-time. The ability to visualize the bridge responses, most typically the bridge vibration movements, in realtime facilitates greatly the understanding of the complex behaviour of the bridge and adds significant engineering value to the monitoring data. Responses of the bridge under normal and severe environmental conditions may be viewed and assessed by operators of the bridge in near real-time with minimal time delay as limited by the network speed. Integrating the real-time data processing modules with the analysis tools to facilitate timely condition assessment of the monitored facility based on evaluation of continuous dynamic monitoring data is the ultimate objective of current research.

\subsection{Data Display Module with Graphical User Interface}

The data display module shown in Figure 5.8 includes extensive plotting capabilities which provide a user friendly and convenient environment to analyze and study the engineering information contained in large data sets. The outputs from the processing module presented in Chapter 4 are stored in an extensive database which contains the outputs of all data that have been collected in the last 6 years. The data display module 
offers rapid viewing with full zooming capabilities, in both time and frequency domain, of any data contained in this extensive database. The data signals used for plotting in the data display module are contained in the structure of processed data matrices created by the processing module. The processed data matrices contain copies of all the data signals at each stage of the processing, thus permitting qualitative performance checks of the processing operations. The user can check the scaling and de-trending operations by plotting the scaled and de-trended signals over the raw signal in both time (Figure 4.5(b)) and frequency domain. The same performance check can be carried out for data patching operation (Figure 4.5(a)), decimation (Figure 4.8) and double integration operations (Figure 4.11). The plots in the frequency domain show the Power Spectral Density estimates of the signals using Welch's method which has been presented in details in Section 5.1.1. Multiple channels in the database can be simultaneously plotted in time and frequency domain allowing quick and insightful comparisons. The displays of the time histories and spectral plots have extremely flexible capabilities, which facilitate the identification or extraction of hidden information and characteristics of the data behaviour. Time history and spectral plots are contained in individual Matlab figures axes which permit a variety of flexible custom presentations of the data. Plots can be easily exported to other modules or applications in common graphic formats for further analysis or presentation.

A specialized algorithm interprets the user inputs from the GUI and performs the following tasks:

- Finds the proper structure of matrices, corresponding to data event chosen by user in GUI, from inside the extensive database. 
- Finds proper matrix, corresponding to user specified type of signal to be plotted, from inside the structure of matrices. One or all of the following: raw acceleration, de-trended and scaled acceleration, patched acceleration, decimated acceleration, velocity or displacements of a data signal may be plotted in a single figure.

- Finds proper data channels to plot. In the GUI, the user chooses from a list of accelerometer labels which channels are to be plotted. The algorithm finds the column inside the data matrix corresponding to the user-specified selection.

- Once the proper channels from the proper matrix are found, they are plotted in the time domain.

- If spectral plots are specified, Welch's PSD estimate with the user specified parameters is computed and plotted.

The visualization of data signatures and spectral plots also provides the user valuable insight into the behaviour of the signals and of the structure's monitoring system itself. The sensors and data acquisition software need regular maintenance. As the monitoring system ages, hardware problems with sensors and data-loggers may occur. The troubleshooting operations performed during routine maintenance operation on the monitoring system are aided by this visualization tool which gives rapid detailed information of signal quality both in the time and frequency domain.

Initial data analysis of modal properties of the structure can be performed by identifying peaks from the PSD estimates using the data display module. This method permits rapid viewing of the structures natural frequencies. Plotting the responses in frequency domain from many events of different excitation scenarios provides a certain understanding of which natural frequencies are more strongly excited by various different loading 
scenarios. One can quickly visually compare the frequency responses from traffic excited events from those excited by wind as shown in Figures 5.2 and 5.3. It is observed that the lower structural modes (below $1 \mathrm{~Hz}$ ) are strongly excited by wind, while those in the 2.5 to $5.0 \mathrm{~Hz}$ and in the 10.0 to $17.0 \mathrm{~Hz}$ range are more sensitive to traffic excitations. Further analysis and research results are presented in Chapter 7.

\subsection{Animation Module with Graphical User Interface}

The animation module has three 3-dimensional structure models of the instrumented section of the bridge, as shown in Figures 6.2 to 6.4. These models are developed in the Matlab environment using patch objects by specifying structural co-ordinates obtained from the design specification drawings. The first model is a simple wire mesh model containing model nodes only at sensor locations. A second model includes more structural detail in the piers and girder cross-sections with the model nodes at sensor locations. The third model is a detailed fine mesh 3D model. The fine meshed 3D model of the bridge has intermediate model nodes between sensor locations for a smoother animation of the dynamic structural responses and vibration mode shapes. The responses at model nodes in the structural models which are not at sensor locations are displayed with geometrically interpolated displacement values from sensor measurement data. The animation of the bridge responses are scaled to aid visualization of the response behaviour.

The responses of the bridge are animated by using the output displacement matrix created by the double integration algorithm included in the processing module discussed in 
Chapter 4. This matrix contains relative displacements for the model nodes at sensor locations. At every sampling instant, the relative displacements are either added or subtracted from the coordinates of the model nodes.

Both the experimental and analytical mode-shapes can be animated using these models. The experimental mode-shapes are determined from operational modal analysis using the SSI module presented in the Chapter 5. A mode-shape matrix is created from the picked solutions when an analysis session is saved in the SSI module. This matrix contains the scaled relative displacements at each sensor locations for the picked modes. Two modeshapes may be simultaneously animated for quick and efficient visual comparison. Two closely spaced modes in frequency may be qualitatively distinguished by the differences in their respective mode-shapes. The operational mode-shapes may be compared to analytical mode-shapes in this module. FEM models of the Confederation Bridge were developed for modal analysis and are presented in Chapter 7. An in-depth verification study discussed in Section 7.2.1 covers the comparison of analytical and operational mode-shapes.

The animation module permits flexible user interactions. Variable parameters of the animations and user options include:

- Play, stop and pause options;

- Dynamic scaling: Displacements may be relatively amplified or scaled down during animations to better visualize lower or higher amplitude vibrations. Current scaling factor is always displayed in the animation window along with the center-span displacements in millimeters; 
- View angle: The 3D model can be rotated in space to view bridge response or mode-shapes from any angle during animations. There are also 3 different pre-set view angles that can be visualized simultaneously and can be switched to and fro during animations;

- Zoom: There is the option to zoom into any part of the bridge to better view motions of individual structural members during animation;

- Playback speed: Animation speed can be manipulated during animation of events from the database but not during real-time animation where animation speed is scaled 1:1;

- Shape capture: A snap shot image, in tiff format, of the bridge model can be captured at any instant of animation.

- Record: A video media file of the animation can be created by pressing the record button during an animation. The video file, with .avi extension, can be played in common media players.

- Displacement plot: Displacement signal from the event being animated can be plotted and visualized inside the animation GUI.

- Sensor locations: A figure showing sensor location and labels can be called from the animation GUI (Figure 6.5).

Responses of the bridge can be animated from a database of recorded events or can be animated in real-time. Real-time algorithms included in both the processing and animation modules detect incoming data from the bridge by use of recursive timer subfunctions that continually search a designated memory path for incoming data. The realtime algorithm imbedded into the processing module handles and redirects the incoming 
data to the processing module where it is subjected to all tasks described in Sections 4.2 and 4.3 of this thesis. The algorithm imbedded in the animation module detects the output from the processing module by use of similar timer sub-routines. The output displacement matrix is then automatically animated in the animation module's GUI (details on code structure for the visualization modules and their real-time engines are included in Appendix E). Real-time animation offers unparallel monitoring during and after significant events such as strong wind, ice floes and earthquakes. It also offers great insight of operational behaviour under ambient excitations. 


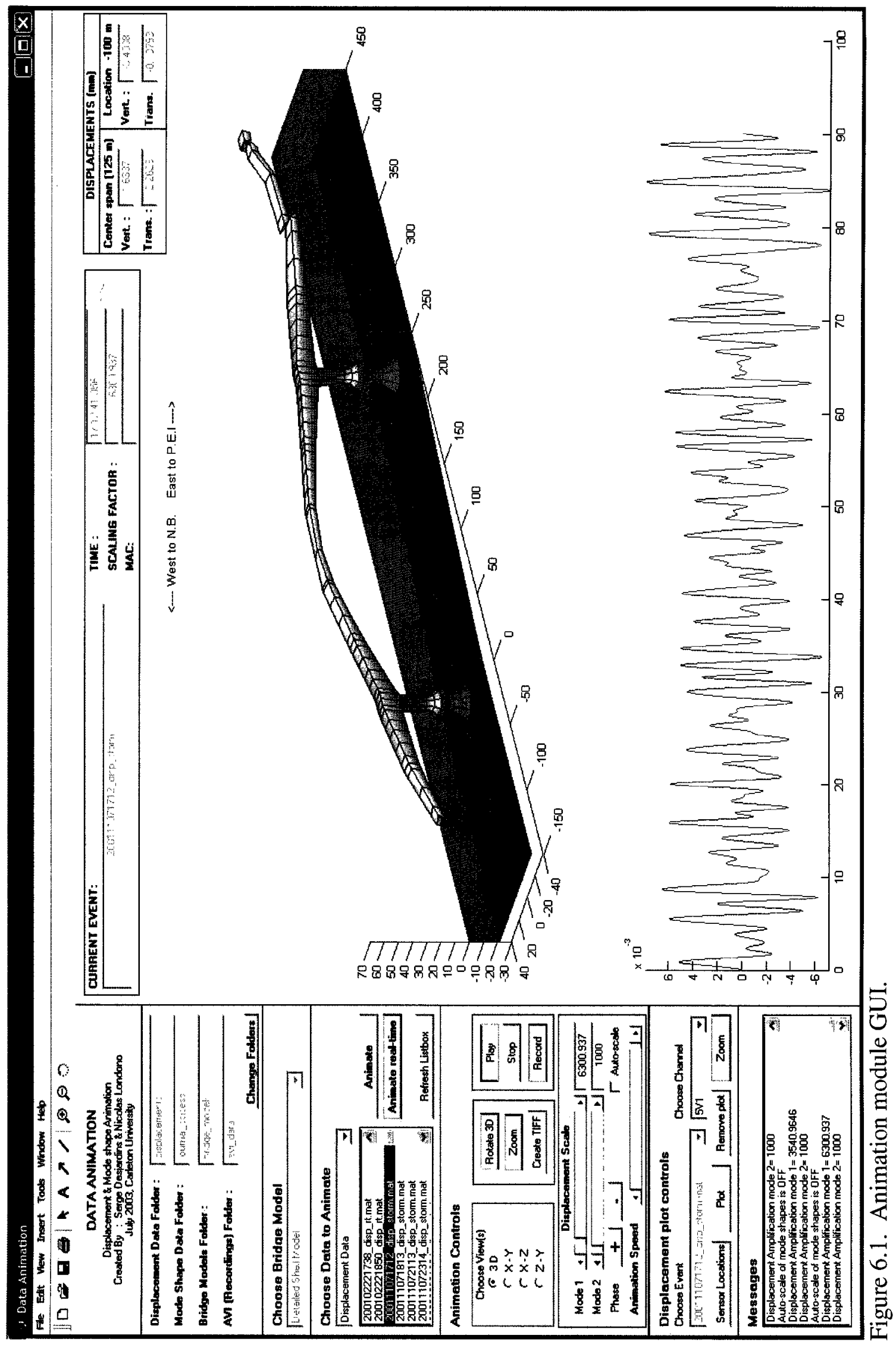




$$
1
$$




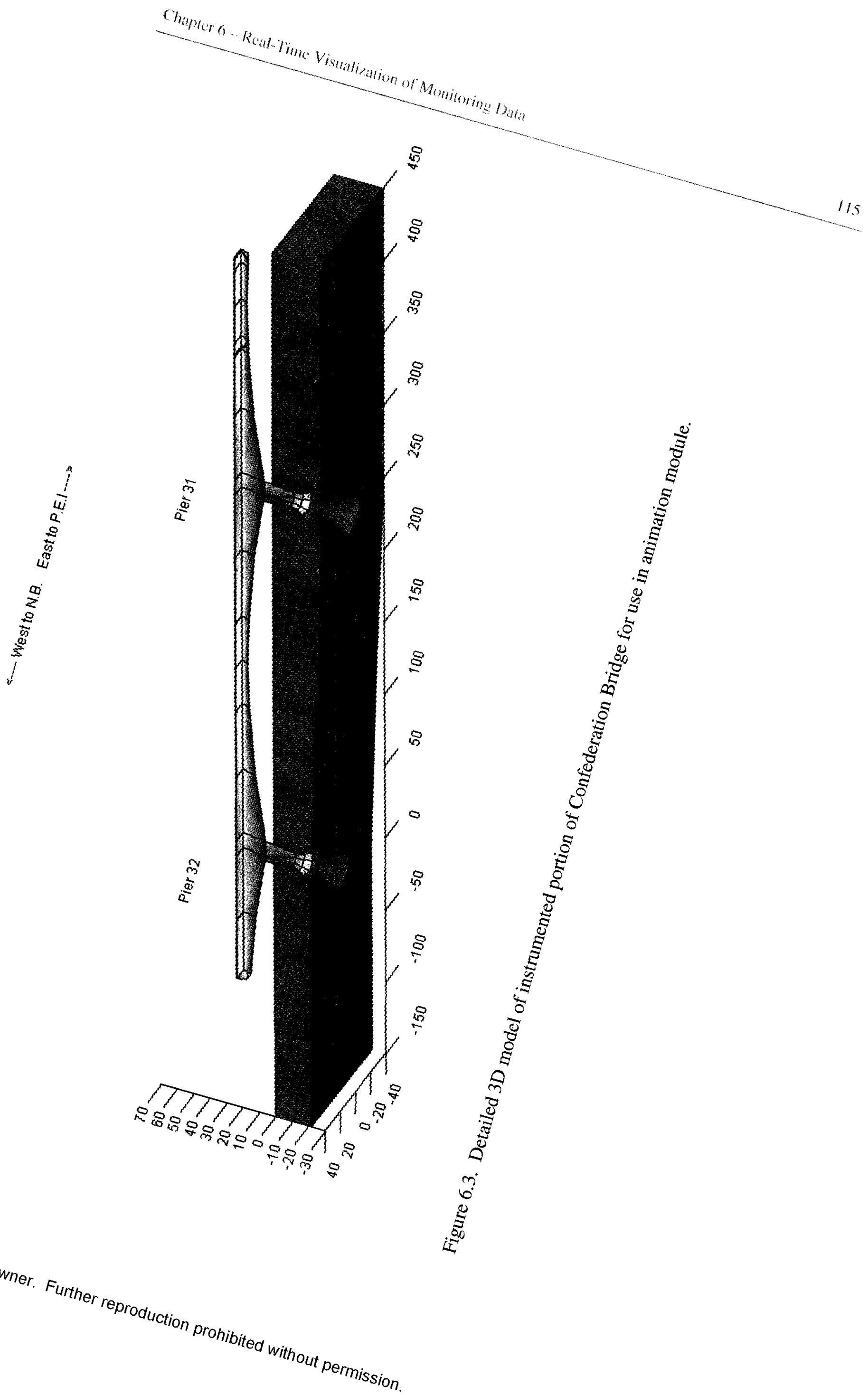




$$
1
$$




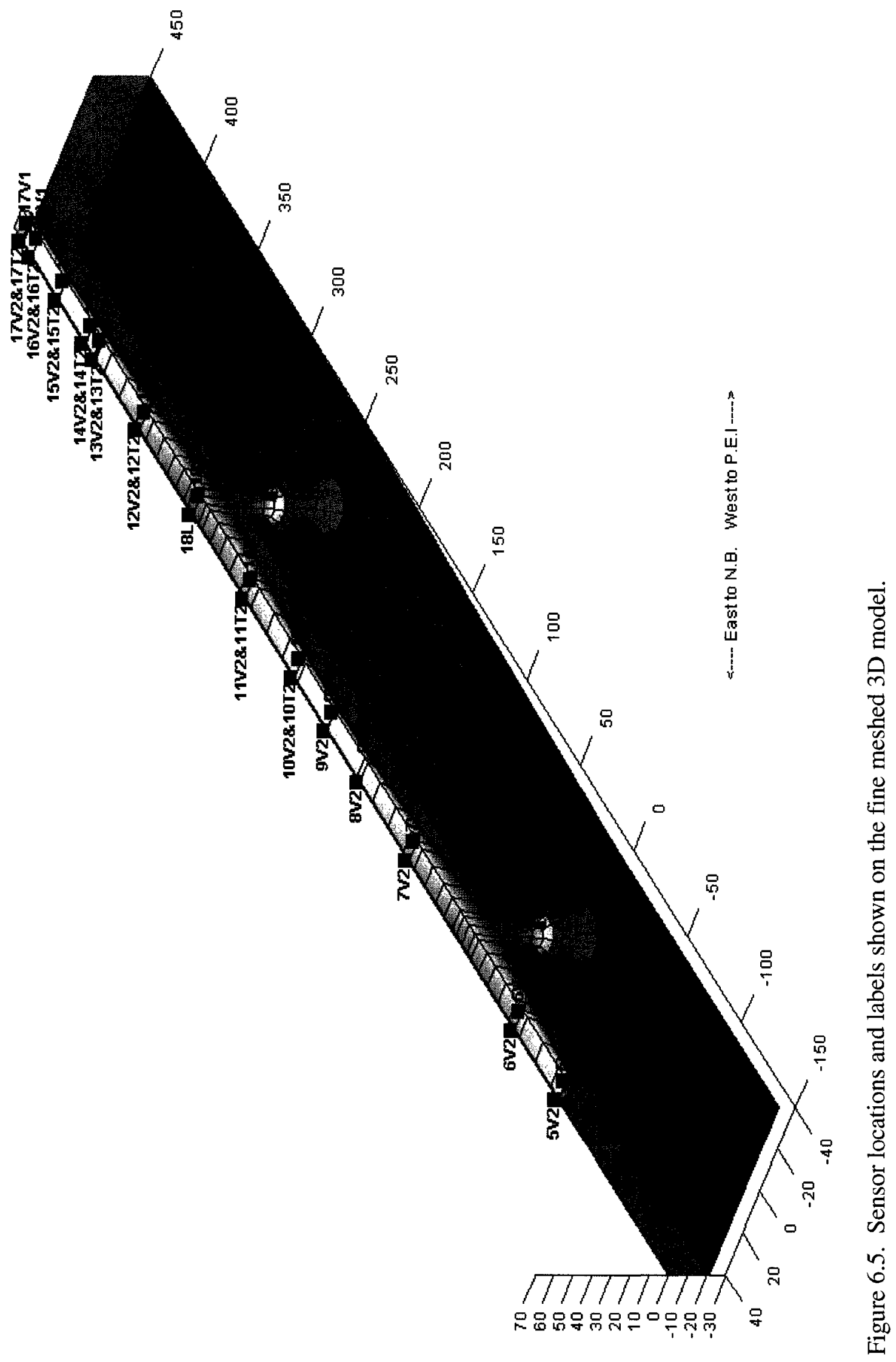




\section{Chapter 7}

\section{Applications of Real-Time Structural Health Monitoring Computer Platform for Dynamic Research on the Confederation Bridge}

This Chapter presents applications of the developed real-time structural health monitoring computer platform and tools for monitoring of the vibration behaviour of the Confederation Bridge. An introduction of a long-term large scale remote monitoring project of the Confederation Bridge has been described in Chapter 3. The organization, management and processing of the Confederation Bridge monitoring data are efficiently handled by the data processing module of the advanced integrated computer platform described in earlier chapters of the thesis. Detailed analysis of the monitoring data and 
the extraction of practical engineering information by advanced system identification techniques have been carried out by using the data analysis modules discussed previously. Understanding of the complex vibration behaviour of the Confederation Bridge is enhanced by using the data visualization modules. For any monitoring project that involves a large volume of data, the need and requirement of efficient advanced computer tools to handle, process and analyze the data and information are clearly demonstrated by the application of the integrated computer platform developed in this thesis for the Confederation Bridge monitoring project. The efficiency, powerful capabilities and user friendly graphical user interfaces are critical to the successful use of the monitoring data. In this Chapter, a brief literature review of previous research on dynamics and behaviour of the Confederation Bridge is presented. Recent important findings obtained using the newly developed tools and engines are then presented. Some very exciting new findings, which have never been reported before, are presented to demonstrate the impact of using the new computer tools in opening up new opportunities of obtaining new information which may affect engineering practice in the future.

\subsection{Previous Research}

The dynamic vibration monitoring system of the Confederation Bridge has been in operation since the bridge opened in 1997. Vibration data has been collected in triggered format since the opening. A large scale pull-test was conducted in April 1997, and controlled traffic tests were carried out in December 2000. The vibration responses of these tests were captured by the dynamic monitoring system described previously in this 
thesis and represent important data sets for in-depth research. The development and calibration of analytical models and research studies carried out with the dynamic data from the Confederation Bridge are discussed here.

\subsubsection{Dynamic Modeling}

A series of Finite Element (FE) bridge models for analytical modal analysis have been developed by various researchers. The FE models attempt to represent analytically the behaviour and response of a representative portion of the Confederation Bridge. The behaviour of the complete portal frame containing the instrumented portion of the structure between Piers 31 and 32, as shown in Figure 3.5, is considered representative of the rest of the structure. Three different FE models of the bridge have been developed to analyze the dynamic behaviour of the Confederation Bridge. These models are described here because of their relevance and importance in later correlations of the analytical results with the field obtained dynamic properties of the bridge.

Jeager et al. (1997) presents a finite element model of the Confederation Bridge for its seismic design analysis. Two and a half portal frames including two drop-in spans are modeled with 821 concentrated mass, 452 beam elements and 395 nodes. Fifty-six separate modes were obtained using the SAP-90 structural analysis program. The first nine modes range from $0.297 \mathrm{~Hz}$ to $0.443 \mathrm{~Hz}$ (Table 7.1).

Lau et al. (2004) present three more analytical models:

- 3D shell elements model for one complete portal frame of the structure,

- 3D beam elements model for one complete portal frame and 
- 3D beam elements model spanning two complete portal frames including a drop-in span.

A 3D finite element model of a complete portal frame in 30 meters of water depth has been developed using the finite element code COSMOS. The girders and piers are modeled with 8-node quadrilateral thin shell elements and 6-node triangular shell elements. The modeling of the interaction of the portal frame with adjacent drop-in spans is considered by adding half the mass of each drop-in span to the supporting overhang in both the transverse and vertical direction and the full mass or no mass in the longitudinal direction, depending if actual connection with drop-in span is hinged or is sliding plate. The nominal value for the modulus of elasticity of concrete is specified for the model as $E_{c}=35 \mathrm{GPa}$. A total of 1292 nodes and 444 elements are included in this FE model. The resulting fundamental frequency is found to be $0.299 \mathrm{~Hz}$ and the first twelve modes range in frequency from $0.299 \mathrm{~Hz}$ to $2.268 \mathrm{~Hz}$ (Table 7.1). The mode-shapes of the first 6 modes are presented in the reference by Lau et al. (2004).

This same portal frame is modeled using 3D beam elements. The geometrical properties of the variable cross-section beam elements are determined from design drawings and calibrated with deformations of the actual member. The interactions with adjacent dropin spans are modeled by means of lumped mass elements at the two ends of the girder. For each structural component, such as girder, pier shaft, pier base and drop-in span, the mass density is calculated from the weight of the component and its volume in the model. The mass of significant non-structural components, such as road barriers and pavement are included as additional masses to the beam elements. However, the stiffness contribution of these components is neglected. The 3D beam model of one portal frame 
contains a total of 52 elements; 40 elements comprising the girder and 6 elements comprising each of the two piers. The beam model, with $E_{c}=35 \mathrm{GPa}$, is verified and calibrated using the shell model results. The first twelve natural frequencies obtained from this model range from $0.299 \mathrm{~Hz}$ to $2.306 \mathrm{~Hz}$ (Table 7.1) and have less than $2 \%$ discrepancy with the results from the 3D shell model (Lau et al. 2004).

To better understand the overall dynamic behaviour of the Confederation Bridge two consecutive portal frames plus a drop-in span between piers 29 and 32 , of the instrumented portion of the bridge, are modeled using 3D beam elements by the finite element code COSMOS, as shown in Figure 7.1. The objective of the investigation of two portal frames of the bridge is to study the interaction behaviour between adjacent repetitive frame units. The two portal-frame beam model is also verified and calibrated using the shell model results. Eighteen beam elements are used in the modeling of the main girder, four for each drop-in span and seven for each pier. In total, the beam model comprises 112 beam elements, 6 mass elements and 113 nodes. Appropriate end release conditions of the beam elements are adopted to model the hinge and sliding plate connections between the drop-in span and the portal frames. Half of the mass of the drop-in span is added at the boundary node in the transverse and vertical directions for the case of sliding plate bearings, while the entire mass of the drop-in is added to the boundary node in the longitudinal direction in the case of a fixed hinge bearing. The results of analysis presented in the reference by Lau et al. (2004) include the 10 first modes ranging in frequency from $0.3 \mathrm{~Hz}$ to $0.67 \mathrm{~Hz}$ (Table 7.1 ). 
These models provide the basis for the verification of experimentally obtained dynamic properties of the Confederation Bridge. They have been and are continually being calibrated with research results from monitoring data analysis.

\subsubsection{Pull Test}

Lau et al. (2004) present the calibration of the two portal frame beam model, shown in Figure 7.1, with experimental data acquired through a dynamic pull-test performed on the Confederation Bridge. The pull-test was conducted in April 1997, two months prior to the official opening of the bridge. A cable was attached above the ice shield of pier 31 at one end and pulled by a test boat at the other. The horizontal load was applied slowly up to a maximum of $1.40 \mathrm{MN}$ and suddenly released. The dynamic monitoring system captured the subsequent "free-vibration" response of the structure. The power spectrum of response measurements from sensor location 9T1 is computed. The FFT of the 20 second data set sampled at $294 \mathrm{~Hz}$ was computed with $2^{17}$ points for FFT. The dominant frequencies were then determined from Lagrangian interpolation through a number of points around the corresponding peak. The dominant frequency is found to be $0.4769 \mathrm{~Hz}$ and the second dominant frequency is found to be $1.3042 \mathrm{~Hz}$ (Table 7.1). The modal free-vibration acceleration time histories of the two identified modes are obtained by digital band-pass filtering the results of FFT around the identified frequency values and then applying the inverse FFT. The pass-band for the digital filters was set wide enough to avoid distortion of the time-histories but narrow enough not to include adjacent vibration modes. The pass-band for the first dominant mode was 0.41 to $0.69 \mathrm{~Hz}$ while the pass-band for the second mode was 1.0 to $1.53 \mathrm{~Hz}$. The damping ratios were 
subsequently obtained from the modal free-vibration time histories and were found to be 0.031 for the first mode and 0.037 for the second (Table 7.1).

With the resulting values of the dynamic properties obtained from the analysis of the pull-test measurement data, the two portal beam model presented in Section 7.1.1 and detailed in the reference by Lau et al. (2004) is calibrated. The material properties of $E_{c}$ and $K_{r f}$, representing the elastic modulus of the structure and the rotational stiffness of the foundation respectively, are updated by trial and error analysis. Different $E_{c}$ and $K_{r f}$ properties of the FE model are specified for each analysis and the resulting dominant frequency is compared with the experimentally obtained dominant frequency $(0.4769 \mathrm{~Hz})$. The values of $E_{c}=34 \mathrm{GPa}$ and $K_{r f}=7.06 \mathrm{MN}-\mathrm{m} / \mu \mathrm{R}$ produce a resulting dominant frequency matching the experimental results are taken as the model's material properties. The average damping ratio (0.034) from the two extracted modes is also adopted in the FE beam model of two portal frames.

\subsubsection{Dynamic Characteristics Obtained From Ambient Excitations}

Naumoski et al. (2002) have studied some ambient condition dynamic monitoring data from the Confederation Bridge to determine the dynamic characteristics of the structure. By ambient data it is meant data sets collected under moderate wind and traffic conditions. Using a total of 8 ambient wind and traffic triggered data sets from January and February 2000 they have identified several modes. Their method for obtaining the dynamic properties (frequencies and mode-shapes only in this case) consists in computing the average Fourier spectra of each signal and picking peaks for the identification of natural frequencies. Band-pass filtering around these identified 
frequencies isolates the modal acceleration time histories. From the acceleration time histories they obtain the modal displacement time histories for the vertical or transverse channels, depending on which mode they intend to shape. Once each displacement time history from all proper measurement locations are obtained and properly normalized before they are animated to show the shapes. The identified vertical modes include 0.7 $\mathrm{Hz}, 1.7 \mathrm{~Hz}, 2.9 \mathrm{~Hz}$, and $4.8 \mathrm{~Hz}$ and the identified transverse modes include $0.5 \mathrm{~Hz}, 0.9$ $\mathrm{Hz}, 1.4 \mathrm{~Hz}, 1.95 \mathrm{~Hz}$, and $2.8 \mathrm{~Hz}$ (Table 7.1). The mode-shapes for these modes can be found in the reference (Naumoski et al. 2002). The torsion modes are identified by computing the Fourier spectra of the record obtained from subtracting the measurement records at each location V1 from the records at each location V2. Two torsion modes were identified, in this manner, at $3.3 \mathrm{~Hz}$ and $4.2 \mathrm{~Hz}$ (Table 7.1). An important observation of the dynamic characteristics is the identification of local vibration modes in the frequency range of 10 to $16 \mathrm{~Hz}$ which will be covered exhaustively by a subsequent study by W.C. Li and others of the Confederation Bridge monitoring team and briefly discussed in Section 7.1.5.

The extracted dynamic characteristics from Naumoski et al. (2002) were compared with those obtained by Jaeger's FE model presented in Section 7.1.1. The correlation of modes seems to have been done qualitatively by observing figures of analytical versus experimental mode-shapes. Naumoski et al. (2002) conclude their analytical comparison by stating that the experimentally extracted frequencies are significantly higher than those obtained by Jaeger et al. (1997) and that more research is needed to fully understand the dynamic response of the structure. 
Zhang (2001) also extracted dynamic properties of the Confederation Bridge from some ambient response data. Advanced system identification with the stochastic subspace method, similar to the one described in Chapter 5, is employed by Zhang. This approach has advantages compared to the procedure by Naumoski et al. (2002) because it considers the noise contamination problem in the monitoring data and tries to minimize its influence on the resulting modal properties. The commercially available software CADA-X is used in the data analysis by Zhang (2001). The data signals are low-pass filtered at $15 \mathrm{~Hz}$ prior to detailed system identification analysis. The two vertical records at a single measurement location were combined by averaging to eliminate the torsion responses. A total of 22 modes were identified in the frequency range of 0 to $5.33 \mathrm{~Hz}$. Table 7.1 lists the identified modes. Mode-shapes are illustrated using a wire geometric model of the bridge (Zhang 2001). Damping ratios are also experimentally obtained and shown in Table 7.1. The system identification technique employed by Zhang permits identification of many modes, some of which are closely spaced and therefore not obtained by Naumoski et al. (2002).

\subsubsection{Controlled Traffic Load Test}

The traffic tests conducted overnight of the 13 to $14^{\text {th }}$ of December 2000 consisted of loaded trucks traveling over the instrumented portion of the bridge at different speeds and different configurations under low wind conditions. The first set of test cases consisted of a single loaded truck traveling at the constant speeds of 40 and $80 \mathrm{~km} / \mathrm{h}$ in the right-hand lane over the instrumented portion. The second set of test cases included two trucks tailgating in a single lane at constant speeds of 40 and $60 \mathrm{~km} / \mathrm{h}$ while the third set of cases 
involved both trucks traveling side-by-side at speeds of 40 and $60 \mathrm{~km} / \mathrm{h}$. Dynamic data were recorded by the high-speed data acquisition system during these tests.

Zhang (2001) presents details of the traffic tests and provides a detailed example of the analysis procedures undertaken with the response data from the three test cases. The subspace identification method, integrated into the software CADA-X, is employed to analyze the traffic test data which produces the dynamic properties listed in Table 7.1. It should be noted that the damping ratios obtained from the monitoring data of ambient wind and traffic excitations and those obtained from the controlled traffic test data as reported by Zhang are similar. This is contrary to the observations in a later study by Londoño and Lau (2003b) which show that the complexity of the damping phenomenon makes it difficult to determine accurately and consistently damping ratio results from vibration monitoring data. The observations on variability of damping ratios are discussed in more details in Section 7.2.

Following the same analysis procedure as described in Section 7.1.3 on the controlled traffic test data with the same processing and analysis methods described in Section 7.1.3, Naumoski et al. (2004) identify a new vertical mode at $1 \mathrm{~Hz}$ (Table 7.1) caused by slower moving vehicles which is not identified in their earlier study (Naumoski et al. 2002).

\subsubsection{Local Vibrations}

The size and dimensions of the box girders and the wide spacing of the webs permit some local vibrations of the box girders under heavy traffic loadings. Local vibrations have been observed by Naumoski et al. (2002) in the frequency range of 10 to $16 \mathrm{~Hz}$. W.C. Li and others of the monitoring research team at Carleton University have placed seven 
extra accelerometers at location 15 during the controlled traffic test described in Section 7.1.4. The subsequent study by this research team states that the response measurement of the extra sensor placed at the center of the deck slab clearly identifies some important local vibration modes. The Fourier amplitude spectrum of the time history responses of the extra sensors during the test case of a single truck traveling at $80 \mathrm{~km} / \mathrm{h}$ clearly shows a dominant mode at $13.5 \mathrm{~Hz}$ and some other less dominant modes at $11.7 \mathrm{~Hz}, 12.7 \mathrm{~Hz}$, and $16.4 \mathrm{~Hz}$. The study further states that these girder box vibrations occurring under traffic excitations are more quickly dissipated than the global vibrations.

An updated 3D shell model with a finer mesh than the one described in Section 7.1.1 has been developed for the study on local vibrations of the box girder cross-sections by $\mathrm{Li}$ and others. The new 3D shell model is developed using finite element code COSMOS and represents one typical bridge portal frame corresponding to the instrumented portion of the structure between Piers 31 and 32 (Figure 7.2). Main girders and piers are modeled using 4 node quadrilateral thin shell elements and 3-node triangular shell elements. The ice shield component of the pier is modeled by 4-node tetrahedron solid elements which have compatible nodal degrees of freedom with the adjacent shell elements. The interaction of the portal frame with the connecting drop-in spans, which are not included in the model, is represented by lumped mass elements. The mass of the drop-in span is equally divided and added to each bearing in the transverse and vertical directions, since all four bearings of a drop-in span prevent motion in these directions. The entire mass of the drop-in span is added in the longitudinal direction of the bearing which prevents longitudinal motion since only one of the four bearings on the drop-in span prevents relative motion in this direction. The dimensions and material properties of 
this model are based on the design drawing dimensions and specifications. The model comprises a total of 2381 elements and 2206 nodes, resulting in a reasonably fine mesh (Figure 7.2). The 3D shell model described here in detail is also the subject of comparison in the design verification studies presented in Sections 7.1.7 and 7.2.1 of this thesis.

The finite element structural model of the bridge was subjected to load simulations to observe the effects of local vibrations. The finer mesh of the 3D shell model permitted observation of the local deformations which occurred at different points along the structure correlating with the location of the moving load. The box-girder contour modeshapes were identified and were found to be varying in amplitude in a longitudinal manner.

\subsubsection{Design Verification}

A detailed design verification study was undertaken by Londoño and Lau (2003a). Data acquired from the dynamic monitoring system was processed and analyzed in order to obtain the modal properties of the Confederation Bridge. Three types of data sets which correspond to different excitation scenarios were chosen for analysis:

- Data sets corresponding to bridge responses due to moderate wind with average speeds of approximately $60 \mathrm{~km} / \mathrm{h}$;

- Data sets corresponding to responses from the controlled traffic tests;

- Data sets corresponding to responses of the bridge due to a major wind storm in November 2001. This wind storm was the event that produced the strongest responses yet recorded at the Confederation Bridge, as of October 2004. 
As an aside, one dynamic data set corresponding to the windstorm was processed and analyzed by Naumoski et al. (2004). No extracted dynamic properties were reported, only maximum dynamic deflections were presented. The largest measured transverse deflection from the data set studied was $13.5 \mathrm{~mm}$ while the maximum vertical deflection was $9.0 \mathrm{~mm}$. It is worth noting here that only one of the four data sets recorded during the windstorm was studied by Naumoski et al. (2004). The other unused three data sets include higher bridge responses recorded during the windstorm.

The complete data sets mentioned above were processed and analyzed by Londoño and Lau (2003a) using Matlab. This early processing engine is the basis of part of the computer platform developed in this thesis. The processed signals were analyzed using the operational modal analysis software CADA-X which integrates a variant of the stochastic subspace identification method detailed in Chapter 5. The identification method incorporated in CADA-X includes a stabilization diagram to differentiate stable solutions from spurious ones. The extracted modal properties from these data sets are included in Table 7.1. A simple geometric wire model was built for visualization of the identified mode-shapes.

The extracted modal properties were used in a verification study of the design parameters and assumptions of the bridge. The FE model used for comparison by Londoño and Lau (2003a) is the 3D beam element model spanning two portal frames including a drop-in span detailed by Lau et al. (2004), discussed in Section 7.1.1 and shown in Figure 7.1. This FE model was also calibrated with data captured from the pull-test detailed in Section 7.1.2. A comprehensive comparison study of experimentally identified and analytical mode-shapes has been carried out (Londoño and Lau 2003a). A modal 
assurance criterion (MAC) was also calculated between the experimental and analytical mode-shapes. An MAC of 1 indicates perfect correlation of the mode-shapes while an MAC of 0 indicates no correlation. Table 7.2 shows the analytical and experimental modes which correlate well and their corresponding MAC values. The experimental modes show a consistent higher frequency than the design value, which implies that the structure is generally stiffer than the model.

This study by Londoño and Lau (2003a) successfully showed the effectiveness of the stochastic subspace identification method in dealing with noisy output only structural response data by successfully identifying modes which correlate well with those previously obtained by FE modeling. An important observation by Londoño and Lau (2003a) is the observed variability in the identified modal parameters. Modal properties extracted from different data sets tend to give slightly different results in identified frequency and damping ratios. The observed variability in the modal parameters complicates the practical use of ambient vibration response data for condition assessment which rely on changes in dynamic characteristics to detect and locate damage.

\subsubsection{Baseline Variability of Modal Properties}

After observing the significant variability of identified parameters in their verification study, Londoño and Lau (2003b) have analyzed a series of data sets with similar loading and environmental conditions to determine the baseline variability of extracted dynamic properties. The level of variability of the structure during the healthy early stage of the bridge is determined in order to be able to use future monitoring data for vibration-based structural condition assessment. By analyzing data sets of similar loading and 
environmental conditions, the identified variability in modal properties represents the baseline, or lower bound, level of variability, assuming no damage occurred during the collection of the data sets which spanned 4 days in this study. The objective of this and future studies is to determine if the identified baseline variability is significant enough to mask any changes in frequency, or other modal properties to prevent their use in condition assessment algorithms for the identification of damage or deterioration.

A total of ten data sets representing bridge behaviour during typical ambient operational conditions were collected over four days in March of 2003. Temperature and wind speeds were similar in all the data sets (Londoño and Lau 2003b). The system identification method used in this study by Londoño \& Lau (2003b) is the same as in their verification study which is a variant of the SSI method incorporated in CADA-X software. The duration of data sets is 10 minutes in this study, which gives more information of the lower frequency modes and a more consistent identification. The analysis results of this study are shown in Table 7.1. The four modes shown are identified from all ten data sets. The extracted frequencies are more consistently obtained than the damping ratio estimates. The four eigenfrequencies were extracted from the ten data sets with standard deviations at or below $0.6 \%$ of the mean as opposed to the damping estimates which have standard deviations between $34 \%$ and $55 \%$ of the mean. The $0.6 \%$ standard deviation of identified frequencies from data sets of similar loading scenarios and environmental conditions shows that it is possible to retrieve highly consistent modal frequencies. Two reasons are presented by Londoño and Lau (2003b) as to why the damping ratios are not as consistently identified. Firstly, the physical damping phenomenon is very complex and may not be accurately represented by the 
structural model. Secondly, the viscous damping modeling assumption is only a practical approximation of the actual damping behaviour. The mode-shapes from a single mode acquired from all ten data sets are compared to each other by averaging the MAC value of all mode-shapes with respect to all other mode-shapes of the identified mode. Average MAC values of the four identified modes of the study are $0.987,0.984,0.990$ and 0.974 respectively. This shows very promising results of consistent identification of modeshape properties. There are some localized discrepancies in mode-shape amplitude observed while correlating the individual amplitudes from all data sets with the amplitudes from the first data set. This may limit the use of monitoring data for use in damage localizing algorithms which rely on localized changes of mode-shapes.

\subsection{Research Results Obtained Using Tools}

In the previous section, VBSHM research of the Confederation Bridge is discussed. Monitoring bridge responses of important loading events have been analyzed by various researchers using different analysis techniques. Analysis of ambient vibration data has also been reported. Advances in research on the behaviour of ambient vibration data are of great importance for successful long-term continuous health monitoring of structures. With the development of the computer tools described in this thesis, more in-depth studies into design verification and variability have been performed. The advantage is the capability of the developed computer platform to efficiently analyze large number of data sets in much shorter time periods. The complete integration of various processing and analysis engines and tools into one central application greatly facilitates the objective 
of data mining. The added ability to visualize aspects of the data, including time histories, signatures or power spectrum and power density characteristics or animations of bridge responses or mode-shapes greatly improves the efficiency of the analysis. The visualization tools are extremely flexible which permits custom user-specified visualization of analysis results. With these new tools, detailed studies involving a large number of data sets have been performed and are presented briefly here.

\subsubsection{In-Depth Design Verification}

The study detailed in this section was performed using the computer tools and modules developed and presented in this thesis for the processing, analysis and visualization of the Confederation Bridge monitoring data. Ten different data sets were chosen for analysis in a more in-depth study into design verification co-authored with Londoño, Lau and Li (Londoño et al. 2004b). The time histories and power spectral density estimates of the 10 data sets are shown in Figures 7.3 and 7.4 respectively. The chosen data sets correspond to the following loading scenarios:

- High winds;

- Controlled traffic test cases of two trucks following each other and traveling side by side;

- Ambient operational conditions with ice cover;

- Ambient operational conditions with no ice cover.

The stochastic subspace identification (SSI) technique, described in Section 5.2, was used to obtain the modal parameters from the chosen data sets and implemented as an algorithm in the system identification module detailed in Section 5.3. 
Two different finite element models, the fine meshed 3D shell element model presented in Section 7.1.5 and shown in Figure 7.2 and the equivalent 3D beam element model spanning two portal frames presented in Section 7.1.1 and shown in Figure 7.1, are used to obtain the theoretical dynamic behaviour of the bridge and are used in the verification study by Londoño et al. (2004b). The sensitivity of the dynamic properties of the bridge to long-term changes in material properties is examined by varying the material properties of the concrete of the bridge. Computer analyses of the bridge models have been carried out using two different values of the Young's modulus $E$ of concrete. The first value of $E_{c}$ is the nominal design value, and the second value of $E_{c}$ is based on the monitoring data and research by the material monitoring group of the project from tests on concrete samples used in the Confederation Bridge (Ghali et al. 2000).

The elastic modulus of the concrete determines the stiffness of the bridge and has a direct effect on its static and dynamic behaviour. Therefore, this material parameter is an important design assumption that must be verified after construction. The target 28 -day strength of concrete in the design specifications was $\mathrm{f}_{c 28}{ }_{c 2}=55 \mathrm{MPa}$ and the target elastic modulus was $E_{c 28}=35 \mathrm{GPa}$. Ghali et al. (2000) tested 24 concrete cylinders taken from two Confederation Bridge batches. The cylinders were tested at the ages of 2, 7, 14, 28, 90 , and 180 days. The average measured value of $f_{c 28}^{\prime}$ was $63 \mathrm{MPa}$ and $E_{c 28}$ was approximately $38.7 \mathrm{GPa}$. Based on the measurements, a best fit equation for the timedependent variation of $E_{c}$ was proposed as follows

$$
\left.E_{c}(t)=E_{c 28} \sqrt{\exp [0.25(1-\sqrt{28 / t})}\right]
$$

in which $E_{c}(t)$ is the elastic modulus of concrete at age $t$, in days, and $E_{c 28}$ is $38.7 \mathrm{GPa}$, approximately the average measured value. The constant of 0.25 in the equation is for 
normal hardening cement, assumed by Ghali et al. (2000) for the Confederation Bridge concrete. The 1000-day value of $E_{c}$ given by this expression is $42.9 \mathrm{GPa}$. In the present study by Londoño et al. (2004b), two values of $E_{c}$ are used in the bridge FE models for comparison purpose: the target 28-day value of $35 \mathrm{GPa}$ and a 3-year value of $43 \mathrm{MPa}$, which is expected to more realistically reflect the actual properties of the Confederation Bridge concrete.

Table 7.3 presents analysis results from the monitoring data sets and results from both the beam and shell finite element models using the two different values for $E_{c}$. The first 23 identified modes with mean frequency values ranging from $0.34 \mathrm{~Hz}$ to $5.13 \mathrm{~Hz}$ are shown with the corresponding modes from the analytical models. The differences, in percentage value, between the experimentally obtained frequencies and those from the two analytical models with the two different modulus values are also shown in Table 7.3. By examining the average differences of all modes, it is shown that there is a better correlation of the system identification results from field monitoring data with the computer predictions using the updated parameter of $E_{c}=43 \mathrm{GPa}(2$ and $-4 \%$ versus $12 \%$ and $6 \%$ ). This confirms the materials monitoring results and the previous observations of consistently higher experimental frequencies which are due to the fact that the structure is actually stiffer than the computer structure model based on nominal material design specifications. Some information regarding the variability of the extracted modal parameters from data sets collected under different loading scenarios and environmental conditions is also presented in Table 7.3. Averages over all 23 modes indicate a frequency range of variability of $5.9 \%$ and a standard deviation of $2 \%$ of the mean frequency from the 10 data sets. Lower modes also tend to be less consistently identified than higher frequency 
modes, which is a very important observation because the lower modes tend to represent the dominant vibration behaviour of the structure. The variability of modal frequency poses a major challenge for identification of damage from vibration data because change in frequency due to stiffness degradation or damage may be masked by the normal variability of the data. However, a $2 \%$ standard deviation from the mean of an extracted modal property from highly uncertain, complex and noisy output-only monitoring data collected at different times under different loading and environmental conditions is a promising result.

The analytical and experimental modes were correlated by comparing both their frequency values and their mode-shape's MAC values. In the comparison of the modeshapes obtained from FE models with those identified from the monitoring data, modal coefficients at sensor locations are obtained by interpolating the modal coefficients at the adjacent beam model nodes. This permits the MAC calculation between experimental and analytical modes and more importantly the visual comparison of mode-shapes using the animation module described in Chapter 6 of this thesis. Two mode-shapes can be simultaneously animated to visualize any discrepancies. Figure 7.5 to 7.19 show examples of using the animation module of comparison between experimentally obtained and analytical mode-shapes (from beam FE model) of identified modes listed in Table 7.3. Some local discrepancies may be observed at the drop-in span where FE modeling assumptions for the bearing and hinged connections may not accurately represent the actual behaviour of the connections. However, the overall close correlation demonstrates the validity of the SSI algorithm to successfully extract mode-shape information from the monitoring data. 
Damping estimates were also successfully extracted from the monitoring data but with a higher variability than the other modal properties (Table 7.3). The complicated nature of the damping phenomenon and the viscous damping assumption in the state-space model detailed in Chapter 5 are most likely the chief contributions to the $55 \%$ average standard deviation of damping ratios from all modes extracted from the 10 data sets.

\subsubsection{Observed Behaviour of the Confederation Bridge}

Average normalized power spectral density estimates are computed in the vertical and transverse directions for the different loading scenarios studied by Londoño et al. (2004b) presented in the previous section. The obtained spectral plots show very clearly the behaviour the bridge under different loading scenarios. Some typical bridge frequencies are more strongly excited under different loading scenarios. It is very important here to distinguish the frequency behaviour from variability of modal parameters. While different loading scenarios excite different modes, the frequency values of these modes should, in theory, be the same with no discrepancy in the case of a healthy structure. Therefore, it is assumed that loading scenarios do not account for the observed variability of the modal parameters but that they do change the frequency behaviour of the response in that they more strongly excite certain modes. Figure 7.20 to 7.23 show the average normalized power spectral densities in the transverse and vertical directions for the four following loading scenarios studied by Londoño et al. (2004b):

- High winds;

- Controlled heavy traffic test;

- Ambient operational conditions with ice cover; 
- Ambient operational conditions with no ice cover.

Loading scenarios involving high winds typically excite lower frequencies in both the vertical and transverse directions; the dominant modes (identified by peak picking from Figure 7.20) being $0.68 \mathrm{~Hz}, 0.64 \mathrm{~Hz}$ and $0.95 \mathrm{~Hz}$ in the vertical and $0.48 \mathrm{~Hz}, 0.33 \mathrm{~Hz}$ and $0.36 \mathrm{~Hz}$ in the transverse directions. The traffic scenarios seem to excite more the frequencies in the range of $2.5 \mathrm{~Hz}$ to $3 \mathrm{~Hz}$ (and local vibration range above $10 \mathrm{~Hz}$ ) with dominant modes (identified by peak picking from Figure 7.21 ) being $3.43 \mathrm{~Hz}, 3.28 \mathrm{~Hz}$ and $3.47 \mathrm{~Hz}$ in the vertical direction and $3.35 \mathrm{~Hz}, 3.39 \mathrm{~Hz}$, and $3.31 \mathrm{~Hz}$ in the transverse direction. Ambient operational conditions involving moderate to calm winds and normal traffic with ice cover tend to excite evenly all the frequency ranges with dominant modes identified by peak picking from Figure 7.22 at $3.82 \mathrm{~Hz}, 2.86 \mathrm{~Hz}$ and $0.66 \mathrm{~Hz}$ in the vertical direction and $0.47 \mathrm{~Hz}, 0.36 \mathrm{~Hz}$ and $3.62 \mathrm{~Hz}$ in the transverse direction. Typical operational conditions with no ice cover represents the most frequent loading scenario experienced by the bridge. The dominant modes picked from peaks of Figure 7.23 are $0.64 \mathrm{~Hz}, 3.27 \mathrm{~Hz}$, and $0.68 \mathrm{~Hz}$ in the vertical direction and $0.50 \mathrm{~Hz}, 0.34 \mathrm{~Hz}$, and $0.47 \mathrm{~Hz}$ in the transverse direction. Although an important peak at $4.16 \mathrm{~Hz}$ in the vertical direction is found only in scenarios involving ice cover, more research needs to be carried out to more fully understand the dynamic behaviour of the bridge during significant ice cover and ice floes.

\subsubsection{Environmental Effects on the Variability of Modal Properties}

Previous studies by Londoño et al. (2003a, 2003b, 2004b) discussed in the earlier sections of this chapter have clearly identified variability in the extracted modal 
parameters from dynamic vibration monitoring data of the Confederation Bridge. This variability is a serious obstacle for the use of ambient field vibration monitoring data for structural condition assessment that relies upon the detection of changes in the dynamic properties. Changes caused by damage or deterioration of the structure may be masked by the normal variability of the data. The potential causes of variation in the dynamic properties can be grouped as follows:

- Environmental Effects (e.g. temperature, wind, moisture);

- Measurement inaccuracies, computational inaccuracies \& deviations from modeling assumptions;

- Non-linearity of the structure;

- Non-stationarity in the measured data;

- Stiffness degradation reflecting deterioration or damage of the structure;

The lower bound level of variability of extracted frequencies has been studied and quantified at a baseline level of $0.6 \%$ standard deviation from the mean by Londoño et al. (2003b). The baseline variability shows that it is possible to retrieve highly consistent modal frequencies and mode-shapes from monitoring data sets of similar loading scenarios and environmental conditions.

Forty-two data sets collected over a period of ten months of bridge operation under varying environmental conditions and loading scenarios have been analyzed to evaluate the potential effect of temperature, cross-section temperature differentials, amplitude of response signal, and wind speed on the identified vibration properties of the Confederation Bridge. This study was co-authored with Londoño and Lau (Londoño et al. 2004a). It is assumed here that during the elapsed period of time between data sets the 
structural condition of the bridge remained unchanged. The data sets used in the study are selected from the dynamic monitoring database based on the average concrete temperatures at the time of acquisition. To avoid bias and give equal weight to all temperatures, one data set is selected per degree wide temperature range between $-18^{\circ} \mathrm{C}$ to $25^{\circ} \mathrm{C}$ of average concrete temperatures. Each of the 10 minute duration data sets used in the study consists of the sampled acceleration responses from 19 sensors at locations 3 to 9 of Figure 3.5. Table 7.4 shows analysis results obtained by system identification with the SSI algorithm described in Chapter 5 of this thesis. The analysis of 42 data sets would have been a daunting task if it were not for the efficiency of the developed modules for processing, analysis and visualization of the data and results. Some of the data sets were analyzed by different users to provide information for a subsequent study on the effect of the user on the variability of extracted parameters, since the final solutions are subjectively picked from a stabilization diagram. The identification of 8 dominant modes and statistical information on their variability is shown in Table 7.5.

A multiple linear regression analysis is carried out to investigate the correlation of the natural modal vibration frequencies with the environmental and amplitude variables. The linear regression model used in this case study is the following

$$
Y_{i}=\beta_{0}+\beta_{1} \cdot x_{1 i}+\ldots+\beta_{k} \cdot x_{k i}+E_{i} \quad \text { for } i=1, \ldots, n
$$

where $n$ is the number of data points, $x_{j}$ are the predictor variables, $\beta_{j}$ the regression coefficients, $Y_{i}$ is the dependent variable and $E_{i}$ is the error. In the present study the vibration modal frequency is the dependent variable, and the average concrete temperature, cross-section temperature differentials, average signal amplitude (RMS), and wind speed are the predictor variables. The results of the regression analysis are 
presented in Table 7.6. The 95\% confidence intervals for the regression coefficients are calculated, which are also presented in Table 7.6. These confidence intervals allow the identification of the variables most significantly correlated with the variations in the frequencies. Highlighted in Table 7.6 are the variables found to have a high correlation with frequency.

The frequencies of the eight different modes considered in this case study are plotted against temperature in Figure 7.24. For the firt time, as can be observed from the plots of Figure 7.24, there is identification of a clear trend of reduction in the vibration frequencies with increase in temperature. This is likely a reflection of the changes in the material properties with temperature, in particular the elastic modulus of concrete. This significant correlation demonstrates the importance of temperature effects on modal properties and that temperature should be considered in any subsequent damage detection algorithm. 


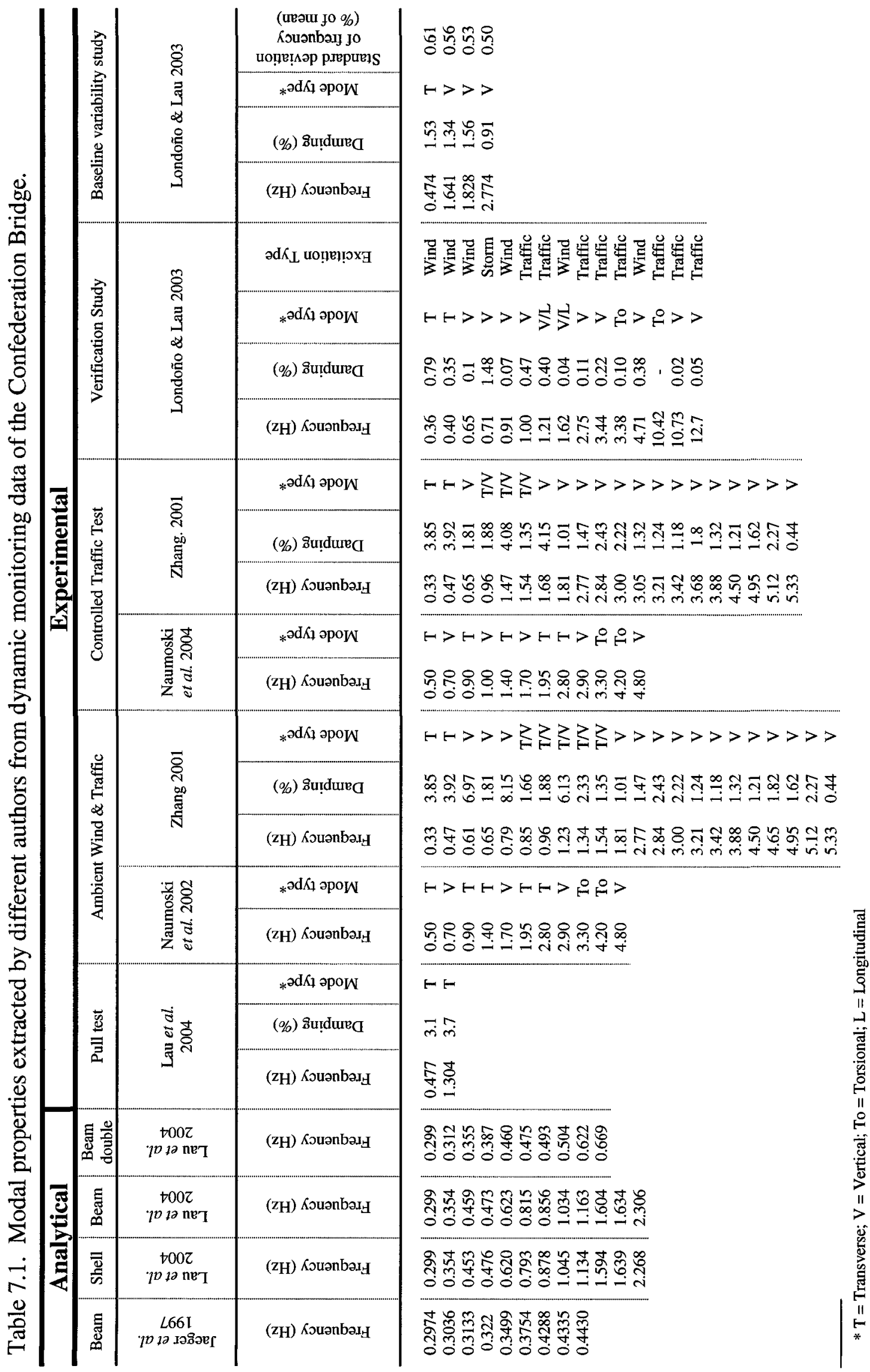


Table 7.2. Verification of experimental modal properties with respect to the analytical results from the beam element model spanning two portal frames (Londoño and Lau 2003a)

\begin{tabular}{cccc}
\hline $\begin{array}{c}\text { Analytical } \\
\text { Frequency }\end{array}$ & $\begin{array}{c}\text { Experimental } \\
\text { Frequency }\end{array}$ & $\begin{array}{c}\text { Modal } \\
\text { Assurance } \\
\text { Criterion }\end{array}$ & Mode type* \\
\hline & & & \\
0.28 & 0.36 & 0.94 & $\mathrm{~T}$ \\
0.33 & 0.40 & 0.93 & $\mathrm{~T}$ \\
0.58 & 0.65 & 0.86 & $\mathrm{~V}$ \\
0.62 & 0.71 & 0.96 & $\mathrm{~V}$ \\
0.79 & 0.91 & 0.94 & $\mathrm{~V}$ \\
0.82 & 1.00 & 0.72 & $\mathrm{~V}$ \\
1.08 & 1.21 & 0.78 & $\mathrm{~V} / \mathrm{L}$ \\
1.60 & 1.62 & 0.83 & $\mathrm{~V} / \mathrm{L}$ \\
2.34 & 2.75 & 0.94 & $\mathrm{~V}$ \\
2.73 & 3.44 & 0.89 & $\mathrm{~V}$ \\
${ }^{*} \mathrm{~T}=$ Transverse; $\mathrm{V}=$ Vertical; To $=$ Torsional; $\mathrm{L}=$ Longitudinal &
\end{tabular}




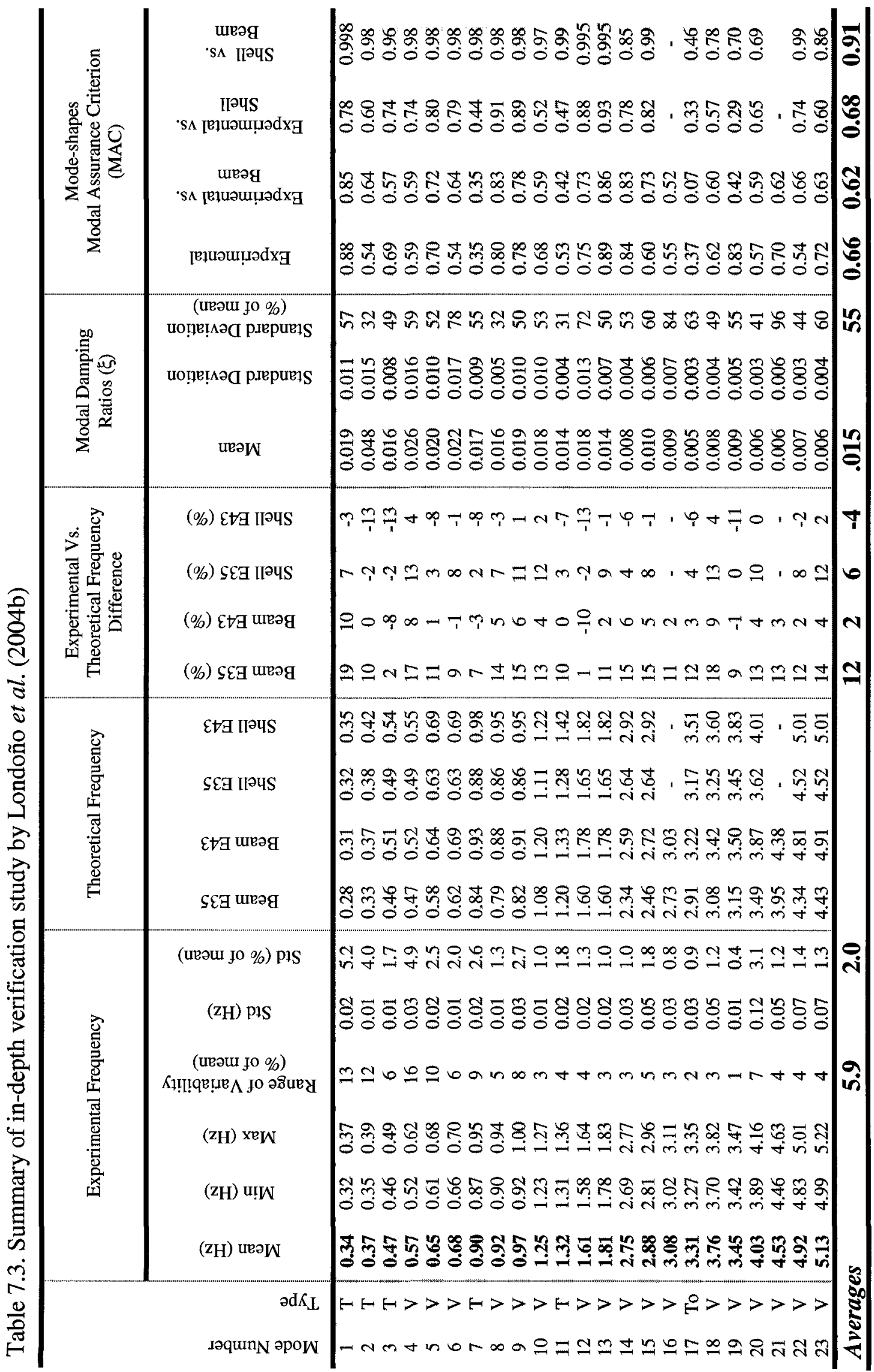


Table 7.4. Analysis results for study of variability due to environmental effects.

\begin{tabular}{|c|c|c|c|c|c|c|c|c|c|c|c|c|c|c|c|c|c|}
\hline $\mathbf{Y}$ & $\mathbf{M}$ & D & H & 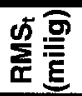 & $\sum_{\underline{x}}^{\infty} \overline{\bar{E}}$ & 虽す & $\begin{array}{l}\Delta \mathrm{T}_{\mathbf{x}} \\
\left({ }^{\circ} \mathrm{C}\right)\end{array}$ & $\begin{array}{l}\Delta \mathrm{T}_{\mathrm{y}} \\
\left({ }^{\circ} \mathrm{C}\right)\end{array}$ & $\begin{array}{l}\mathbf{W S} \\
\mathrm{n} / \mathrm{s})\end{array}$ & $\begin{array}{c}\text { Mode } \\
1\end{array}$ & $\begin{array}{c}\text { Mode } \\
2\end{array}$ & $\begin{array}{c}\text { Mode } \\
3\end{array}$ & $\begin{array}{c}\text { Mode } \\
4\end{array}$ & $\begin{array}{c}\text { Mode } \\
5\end{array}$ & $\begin{array}{c}\text { Mode } \\
6\end{array}$ & $\begin{array}{c}\text { Mode } \\
7\end{array}$ & $\begin{array}{c}\text { Mode } \\
8\end{array}$ \\
\hline 2003 & 3 & 10 & 11 & 0.60 & 0.42 & -5.1 & 0.91 & -3.9 & 6.3 & 0.475 & 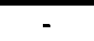 & 1.639 & 1.846 & 2.772 & 3.465 & 4.748 & 5.159 \\
\hline 2003 & 5 & 26 & 23 & 0.63 & 0.31 & 11.0 & -0.49 & 4.2 & 1.6 & - & 0.666 & 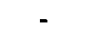 & 1.820 & 2.734 & 3.338 & 4.475 & 5.143 \\
\hline 2003 & 5 & 30 & 8 & 1.10 & 0.56 & 12.9 & 0.10 & 4.0 & 5.1 & 0.468 & 0.658 & - & 1.820 & 2.741 & 3.438 & 4.477 & 5.118 \\
\hline 2003 & 5 & 30 & 8 & 1.10 & 0.55 & 12.9 & 0.10 & 4.0 & 5.1 & 0.470 & 0.665 & 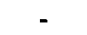 & 1.834 & 2.724 & 3.381 & 4.490 & 5.110 \\
\hline 2003 & 6 & 17 & 13 & 0.76 & 0.54 & 14.3 & -0.27 & 6.0 & 0.7 & - & 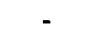 & 1.620 & 1.814 & 2.733 & 3.432 & 4.697 & 5.124 \\
\hline 2003 & 6 & 23 & 9 & 1.14 & 0.57 & 21.2 & 0.47 & 9.2 & 0.4 & 0.472 & - & 1.619 & 1.805 & 2.717 & 3.153 & - & 5.094 \\
\hline 2003 & 7 & 18 & 9 & 0.87 & 0.37 & 21.6 & 0.39 & 5.8 & 2.3 & 0.466 & 0.664 & 1.611 & 1.789 & 2.749 & 3.322 & 4.513 & 5.159 \\
\hline 2003 & 8 & 13 & 22 & 0.39 & 0.25 & 24.0 & 0.22 & 10.1 & 0.7 & - & 0.635 & - & 1.727 & - & 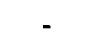 & 4.699 & 5.027 \\
\hline 2003 & 8 & 21 & 13 & 0.84 & 0.51 & 23.0 & 0.06 & 8.3 & 6.7 & - & - & - & 1.798 & 2.742 & - & - & 5.090 \\
\hline 2003 & 8 & 25 & 9 & 0.75 & 0.53 & 17.0 & 0.07 & 2.3 & 12.8 & 0.465 & 0.667 & - & - & 2.718 & 3.269 & 4.627 & 5.121 \\
\hline 2003 & 8 & 25 & 9 & 0.75 & 0.53 & 17.0 & 0.07 & 2.3 & 12.8 & 0.478 & 0.654 & 1.620 & 1.788 & - & 3.296 & 4.478 & 5.095 \\
\hline 2003 & 9 & 11 & 19 & 0.80 & 0.33 & 17.3 & -0.41 & 6.9 & 0.2 & 0.462 & 0.667 & - & - & 2.733 & 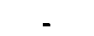 & 4.610 & 5.088 \\
\hline 2003 & 9 & 11 & 19 & 0.80 & 0.33 & 17.3 & -0.41 & 6.9 & 0.2 & - & - & - & 1.750 & 2.725 & - & 4.588 & 5.076 \\
\hline 2003 & 9 & 12 & 18 & 0.79 & 0.26 & 18.4 & -0.21 & 7.5 & 2.6 & - & 0.652 & - & 1.778 & 2.716 & - & - & 156 \\
\hline 2003 & 9 & 25 & 22 & 0.63 & 0.39 & 20.3 & 0.82 & 6.4 & 2.6 & 0.453 & 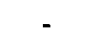 & 1.612 & 1.817 & 2.711 & 3.431 & - & - \\
\hline 2003 & 9 & 25 & 22 & 0.63 & 0.39 & 20.3 & 0.82 & 6.4 & 2.6 & - & - & - & & 2.759 & 3.351 & 4.551 & 5.094 \\
\hline 2003 & 9 & 25 & 22 & 0.63 & 0.39 & 20.3 & 0.82 & 6.4 & 2.6 & - & 0.662 & - & 1.728 & - & 3.385 & 4.551 & 5.158 \\
\hline 2003 & 9 & 26 & 11 & 0.83 & 0.42 & 19.7 & 1.02 & 4.7 & 1.5 & - & 0.655 & - & 1.797 & 2.811 & 3.337 & 4.565 & 5.161 \\
\hline 2003 & 10 & 16 & 7 & 0.59 & 0.33 & 13.8 & 0.71 & 3.8 & 5.0 & - & 0.655 & - & 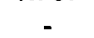 & 2.748 & 3.289 & 4.563 & 5.055 \\
\hline 2003 & 10 & 17 & 13 & 0.95 & 0.51 & 11.5 & 0.64 & 3.0 & 6.4 & 0.472 & - & - & - & 2.724 & & 4.598 & 5.153 \\
\hline 2003 & 10 & 19 & 16 & 0.56 & 0.26 & 9.1 & -0.02 & 3.1 & 6.2 & .486 & 0.647 & - & - & 2.753 & - & - & - \\
\hline 2003 & 10 & 24 & 23 & 0.49 & 0.28 & 7.0 & 0.46 & 1.0 & 15.4 & 0.465 & 0.676 & - & 1.807 & - & - & 4.587 & 5.100 \\
\hline 2003 & 11 & 6 & 20 & 0.88 & 0.57 & 8.6 & 0.25 & 3.0 & 0.8 & - & 0.655 & - & 1.805 & 2.754 & 3.416 & 4.652 & 5.110 \\
\hline 2003 & 11 & 6 & 20 & 0.88 & 0.57 & 8.6 & 0.25 & 3.0 & 0.8 & - & 0.659 & - & - & 2.752 & 3.346 & 4.659 & 5.131 \\
\hline 2003 & 11 & 9 & 5 & 1.04 & 0.66 & 1.8 & -0.12 & -1.8 & 18.9 & 0.470 & 0.663 & - & - & 2.779 & 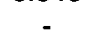 & - & - \\
\hline 2003 & 11 & 13 & 16 & 1.27 & 0.71 & 4.7 & 0.05 & 1.4 & 16.9 & 0.476 & 0.667 & - & 1.796 & - & - & - & 5.118 \\
\hline 2003 & 11 & 26 & 13 & 0.97 & 0.60 & 5.2 & 2.04 & -0.2 & 5.6 & 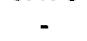 & 0.653 & - & 1.812 & 2.787 & 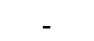 & 4.633 & 5.190 \\
\hline 2003 & 11 & 28 & 8 & 0.72 & 0.39 & 4.0 & 0.52 & 0.0 & 8.8 & 0.471 & 0.659 & - & - & 2.756 & - & 4.682 & 5.109 \\
\hline 2003 & 11 & 28 & 8 & 0.72 & 0.39 & 4.0 & 0.52 & 0.0 & 8.8 & - & 0.664 & 1.603 & 1.821 & 2.761 & 3.432 & 4.684 & 5.187 \\
\hline 2003 & 11 & 29 & 13 & 0.53 & 0.47 & 7.2 & 0.02 & 2.9 & 18.7 & .475 & 0.664 & - & . & 2.785 & - & - & - \\
\hline 2003 & 11 & 29 & 13 & 0.53 & 0.47 & 7.2 & 0.02 & 2.9 & 18.7 & 0.475 & 0.671 & - & - & - & - & 4.639 & 5.126 \\
\hline 2003 & 12 & 4 & 2 & 0.81 & 0.51 & -2.7 & 0.14 & -2.5 & 18.4 & 0.473 & 0.674 & - & - & 2.812 & - & 4.813 & 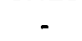 \\
\hline 2003 & 12 & 9 & 10 & 0.87 & 0.47 & -0.4 & -0.05 & -0.1 & 2.6 & 0.467 & 0.672 & - & 1.832 & 2.758 & 3.387 & 4.671 & 5.166 \\
\hline 2003 & 12 & 12 & 2 & 0.60 & 0.39 & 0.5 & 0.56 & 0.3 & 19.9 & 0.473 & 0.671 & - & 1.795 & 2.769 & 3.412 & - & 5.107 \\
\hline 2003 & 12 & 12 & 2 & 0.60 & 0.39 & 0.5 & 0.56 & 0.3 & 19.9 & 0.473 & 0.668 & - & 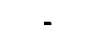 & 2.759 & 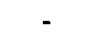 & - & - \\
\hline 2003 & 12 & 12 & 2 & 0.60 & 0.39 & 0.5 & 0.56 & 0.3 & 19.9 & - & 0.651 & - & - & - & - & - & - \\
\hline 2003 & 12 & 12 & 19 & 0.64 & 0.53 & 2.1 & -0.05 & 1.3 & 12.2 & 0.476 & 0.669 & 1.625 & 1.826 & 2.775 & 3.365 & - & - \\
\hline 2003 & 12 & 15 & 17 & 0.42 & 0.39 & -3.7 & 0.00 & -1.3 & 5.4 & 0.480 & - & 1.628 & 1.847 & 2.820 & 3.454 & 4.723 & 5.223 \\
\hline 2003 & 12 & 15 & 17 & 0.42 & 0.39 & -3.7 & 0.00 & -1.3 & 5.4 & 0.479 & 0.677 & 1.632 & 1.859 & - & - & - & - \\
\hline 2003 & 12 & 16 & 8 & 1.03 & 0.60 & -1.6 & -0.24 & 0.2 & 20.5 & 0.480 & 0.651 & - & 1.820 & - & - & 4.696 & 5.181 \\
\hline 2004 & 1 & 7 & 16 & 0.78 & 0.49 & -6.1 & 0.83 & -3.6 & 9.0 & 0.473 & 0.671 & 1.634 & 1.833 & 2.793 & - & 4.739 & 5.205 \\
\hline 2004 & 1 & 8 & 7 & 0.78 & 0.55 & -9.1 & 0.12 & -4.9 & 16.7 & 0.474 & 0.675 & 1.642 & 1.842 & 2.800 & - & 4.729 & 5.196 \\
\hline 2004 & 1 & 9 & 0 & 0.68 & 0.42 & -13.6 & -0.06 & -7.0 & 19.4 & 0.474 & 0.685 & 1.651 & 1.843 & 2.797 & 3.464 & - & 5.158 \\
\hline 2004 & 1 & 10 & 22 & 0.47 & 0.42 & -14.5 & 0.03 & -5.2 & 8.6 & 0.477 & 0.684 & 1.662 & 1.847 & - & - & 4.768 & - \\
\hline 2004 & 1 & 13 & 10 & 0.85 & 0.55 & -12.4 & 0.81 & -4.0 & 2.7 & 0.492 & - & 1.644 & 1.832 & 2.798 & - & 4.776 & 5.259 \\
\hline 2004 & 1 & 14 & 5 & 0.75 & 0.50 & -10.9 & -0.07 & -2.7 & 19.9 & 477 & 0.675 & 1.625 & 1.827 & 2.816 & 3.492 & 4.730 & 5.264 \\
\hline 2004 & 1 & 14 & 5 & 0.75 & 0.50 & -10.9 & & -2.7 & 19.9 & 479 & 0.660 & 610 & 1.832 & & 3.434 & 4.704 & 5.256 \\
\hline 2004 & 1 & 14 & 20 & 0.70 & 0.70 & -15.9 & & -6.1 & 14.7 & .474 & 0.668 & - & 1.869 & 2.803 & $=$ & - & $=$ \\
\hline 2004 & 1 & 14 & 20 & 0.70 & 0.70 & 9 & & & 14.7 & 0.470 & 0.668 & 1.666 & 1.839 & - & - & 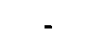 & 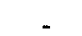 \\
\hline 2004 & 1 & 14 & 20 & 0.70 & 0.70 & -15.9 & -0 & -6.1 & 14.7 & 173 & & - & 1.850 & 2.798 & 3.456 & - & - \\
\hline 2004 & 1 & 14 & 22 & 0.40 & 0.46 & -16.8 & -0.52 & -6.6 & 13.7 & 473 & 0.676 & - & 1.843 & 2.807 & - & - & - \\
\hline 2004 & 1 & 14 & 22 & 0.40 & 0.46 & -16.8 & -0 & -6.6 & 13.7 & 176 & & - & 1.851 & 2.813 & - & - & - \\
\hline 2004 & 1 & 16 & 23 & 0.67 & 0.54 & -17.5 & & -4.5 & 18.5 & 182 & & 1.642 & 1.849 & 2.816 & - & - & - \\
\hline 2004 & 1 & 17 & 11 & 1.02 & 0.60 & -10.9 & 0. & -0.2 & 16.7 & 193 & & - & 1.852 & - & - & - & - \\
\hline 2004 & 1 & 17 & 18 & 0.88 & 0.46 & -8.2 & -0.01 & 1.0 & 21.5 & 0.481 & 0.662 & - & 1.852 & 2.780 & - & - & - \\
\hline 2004 & 1 & 17 & 18 & 0.88 & 0.46 & -8.2 & -0.01 & 1.0 & 21.5 & 0.480 & 0.666 & 1.640 & 1.827 & 2.785 & 3.458 & - & - \\
\hline 2004 & 1 & 17 & 20 & 0.66 & 0.32 & -7.7 & -0.04 & 1.0 & 20.4 & 0.485 & 0.683 & 1.662 & 1.821 & - & - & 4.681 & .241 \\
\hline 2004 & 1 & 20 & 13 & 0.74 & 0.62 & -4.0 & -0.14 & -1.1 & 14.2 & 0.472 & 0.659 & 1.653 & 1.814 & - & 3.410 & - & . \\
\hline 2004 & 1 & 20 & 13 & 0.74 & 0.62 & -4.0 & -0.14 & -1.1 & 14.2 & 0.473 & 0.663 & 1.634 & 1.842 & 2.795 & 3.417 & - & 5.068 \\
\hline
\end{tabular}


Table 7.5. Variability of results from analysis of 42 data sets for environmental effects study (Londoño et al. 2004a)

\begin{tabular}{lcccccccc} 
& Mode & Mode & Mode & Mode & Mode & Mode & Mode & Mode \\
& 1 & 2 & 3 & 4 & 5 & 6 & 7 & 8 \\
\hline $\begin{array}{l}\text { Frequency average } \\
\text { (Hz) }\end{array}$ & 0.474 & 0.665 & 1.634 & 1.820 & 2.769 & 3.387 & 4.641 & 5.143 \\
\hline std $(\mathrm{Hz})$ & 0.007 & 0.010 & 0.018 & 0.031 & 0.033 & 0.074 & 0.093 & 0.057 \\
\hline std $(\%$ of mean) & 1.5 & 1.5 & 1.1 & 1.7 & 1.2 & 2.2 & 2.0 & 1.1 \\
\hline $\begin{array}{l}\text { Damping average } \\
\xi(\%)\end{array}$ & 2.641 & 2.945 & 2.103 & 2.637 & 1.583 & 2.257 & 2.672 & 2.283 \\
\hline std $(\xi \%)$ & 3.177 & 1.321 & 1.344 & 1.900 & 0.684 & 1.529 & 1.449 & 0.931 \\
\hline std $(\xi \%$ of mean) & 120 & 45 & 64 & 72 & 43 & 68 & 54 & 41 \\
\hline MAC average & 0.948 & 0.944 & 0.982 & 0.957 & 0.954 & 0.878 & 0.899 & 0.925
\end{tabular}


Table 7.6. Results of regression analysis in environmental variability study.

\begin{tabular}{|c|c|c|c|c|c|c|c|c|}
\hline & & \\
\hline & & $\beta_{0}$ & $\beta_{\mathrm{RMSv}}$ & $\beta_{\mathrm{RMSt}}$ & $\beta_{T}$ & $\beta_{\Delta \mathbf{T} \mathbf{x}}$ & $\beta_{\Delta \mathbf{T y}}$ & $\beta_{\text {Wind Sp. }}$ \\
\hline 0.47 & $\beta$ & 0.473 & 0.487 & -0.544 & -0.000810 & 0.00250 & 0.00142 & 0.000042 \\
\hline $\mathrm{Hz}$ & $\begin{array}{l}95 \% \text { c.i. for } \beta \\
95 \% \text { c.i. for }\end{array}$ & $\begin{array}{c}0.009 \\
2.0\end{array}$ & $\begin{array}{c}1.407 \\
289\end{array}$ & $\begin{array}{c}2.634 \\
484\end{array}$ & $\begin{array}{r}0.000403 \\
50 \\
\end{array}$ & $\begin{array}{c}0.00542 \\
216\end{array}$ & $\begin{array}{c}0.00125 \\
88\end{array}$ & $\begin{array}{c}0.000317 \\
758\end{array}$ \\
\hline 0.67 & $\beta$ & 0.677 & 0.867 & -3.897 & 0.000139 & -0.00336 & -0.00224 & 0.000096 \\
\hline $\begin{array}{l}\mathbf{0 . 0 \prime} \\
\mathrm{Hz}\end{array}$ & $\begin{array}{l}95 \% \text { c.i. for } B \\
95 \% \text { c.i. for }\end{array}$ & $\begin{array}{c}0.012 \\
1.7 \\
\end{array}$ & $\begin{array}{c}1.752 \\
202 \\
\end{array}$ & $\begin{array}{c}3.163 \\
81\end{array}$ & $\begin{array}{c}0.000559 \\
402\end{array}$ & $\begin{array}{c}0.00545 \\
162\end{array}$ & $\begin{array}{c}0.00165 \\
73 \\
\end{array}$ & $\begin{array}{c}0.000425 \\
445\end{array}$ \\
\hline 1.6 & $B$ & 1.633 & 0.642 & 0.277 & -0.001167 & -0.00991 & 0.00018 & -0.00052 \\
\hline $\begin{array}{l}1.0 \\
\mathrm{~Hz}\end{array}$ & $\begin{array}{l}95 \% \text { c.i. for } \beta \\
95 \% \text { c.i. for }\end{array}$ & $\begin{array}{c}0.043 \\
2.6\end{array}$ & $\begin{array}{l}6.720 \\
1047\end{array}$ & $\begin{array}{c}10.007 \\
3608\end{array}$ & $\begin{array}{r}0.001462 \\
125\end{array}$ & $\begin{array}{c}0.02354 \\
238\end{array}$ & $\begin{array}{c}0.00425 \\
2409\end{array}$ & $\begin{array}{c}0.00148 \\
284\end{array}$ \\
\hline 1.8 & $\beta$ & 1.809 & 2.409 & 1.088 & -0.001623 & -0.00358 & -0.00161 & -0.00087 \\
\hline $\begin{array}{l}\mathrm{Hz} \\
\mathrm{Hz}\end{array}$ & $\begin{array}{l}95 \% \text { c.i. for } \beta \\
95 \% \text { c.i. for }\end{array}$ & $\begin{array}{c}0.030 \\
1.6\end{array}$ & $\begin{array}{r}4.680 \\
194\end{array}$ & $\begin{array}{c}8.100 \\
744\end{array}$ & $\begin{array}{c}0.001541 \\
95\end{array}$ & $\begin{array}{c}0.01334 \\
373\end{array}$ & $\begin{array}{c}0.00426 \\
265\end{array}$ & $\begin{array}{l}0.00119 \\
\quad 137\end{array}$ \\
\hline 2.8 & $B$ & 2.776 & -3.629 & 3.996 & -0.001522 & 0.01133 & -0.00068 & 0.00045 \\
\hline $\begin{array}{l}2.0 \\
\mathrm{~Hz}\end{array}$ & $\begin{array}{l}95 \% \text { c.i. for } \beta \\
95 \% \text { c.i. for }\end{array}$ & $\begin{array}{c}0.034 \\
1.2\end{array}$ & $\begin{array}{c}4.937 \\
136\end{array}$ & $\begin{array}{c}7.981 \\
200\end{array}$ & $\begin{array}{c}0.001600 \\
105\end{array}$ & 0.01254 & $\begin{array}{c}0.00443 \\
655\end{array}$ & $\begin{array}{l}0.00111 \\
244\end{array}$ \\
\hline 3. & $\beta$ & 3.484 & -4.798 & -4.114 & -0.005784 & 0.02017 & 0.00162 & -0.00176 \\
\hline $\begin{array}{l}\mathrm{J}+\mathrm{H}_{\mathrm{H}} \\
\mathrm{Hz}\end{array}$ & $\begin{array}{l}95 \% \text { c.i. for } \beta \\
95 \% \text { c.i. for }\end{array}$ & $\begin{array}{c}0.162 \\
4.6\end{array}$ & $\begin{array}{c}19.288 \\
402\end{array}$ & $\begin{array}{c}34.297 \\
834\end{array}$ & $\begin{array}{c}0.005883 \\
102\end{array}$ & $\begin{array}{c}0.07341 \\
364\end{array}$ & $\begin{array}{c}0.01705 \\
1053\end{array}$ & $\begin{array}{l}0.00481 \\
274\end{array}$ \\
\hline & $\beta$ & 4.716 & -21.123 & 28.288 & -0.008383 & 0.01449 & 0.00836 & -0.00142 \\
\hline $\begin{array}{l}4.0 \\
\mathrm{~Hz}\end{array}$ & $\begin{array}{l}95 \% \text { c.i. for } \beta \\
95 \% \text { c.i. for }\end{array}$ & $\begin{array}{c}0.125 \\
2.7\end{array}$ & $\begin{array}{r}19.150 \\
91\end{array}$ & $\begin{array}{c}37.231 \\
132 \\
\end{array}$ & $\begin{array}{c}0.005989 \\
71\end{array}$ & $\begin{array}{c}0.05114 \\
353\end{array}$ & $\begin{array}{c}0.01856 \\
222 \\
\end{array}$ & $\begin{array}{c}0.00404 \\
284\end{array}$ \\
\hline & $B$ & 5.165 & 7.829 & -11.649 & $-0,004924$ & 0.01885 & 0.00253 & -0.00057 \\
\hline $\mathbf{H z}$ & $\begin{array}{l}95 \% \text { c.i. for } B \\
95 \% \text { c.i. for }\end{array}$ & $\begin{array}{c}0.073 \\
1.4\end{array}$ & $\begin{array}{c}11.697 \\
149\end{array}$ & $\begin{array}{c}21.009 \\
180\end{array}$ & $\begin{array}{c}0.003836 \\
78\end{array}$ & $\begin{array}{c}0.03287 \\
174\end{array}$ & $\begin{array}{c}0.01093 \\
433\end{array}$ & $\begin{array}{l}0.00265 \\
469\end{array}$ \\
\hline
\end{tabular}


m1

$\mathrm{m} 2$
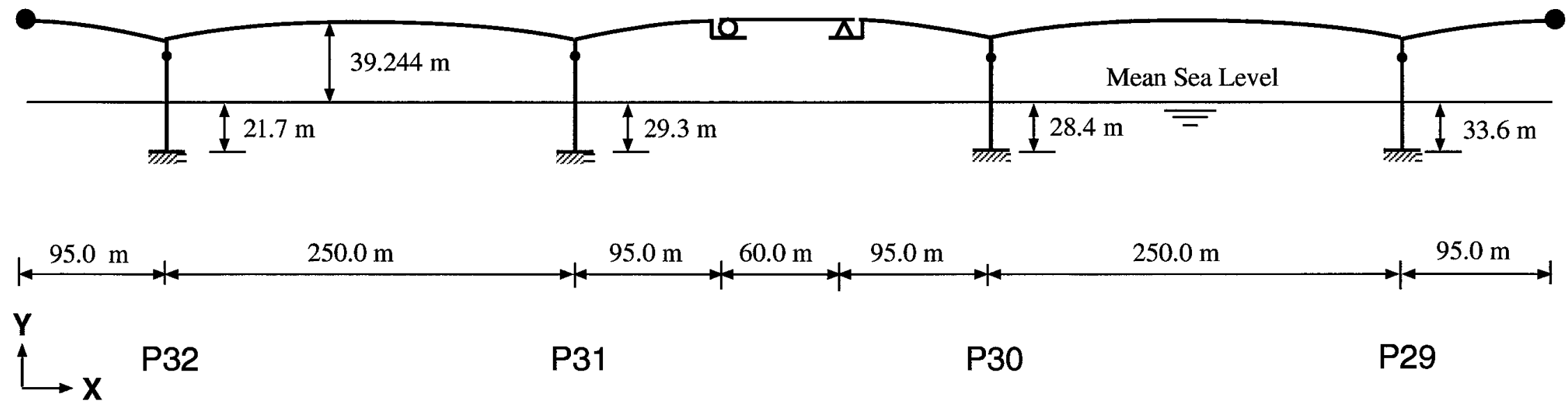

Figure 7.1. Finite element model using 3D beam elements spanning two portal frames of the Confederation Bridge. 


$$
F
$$




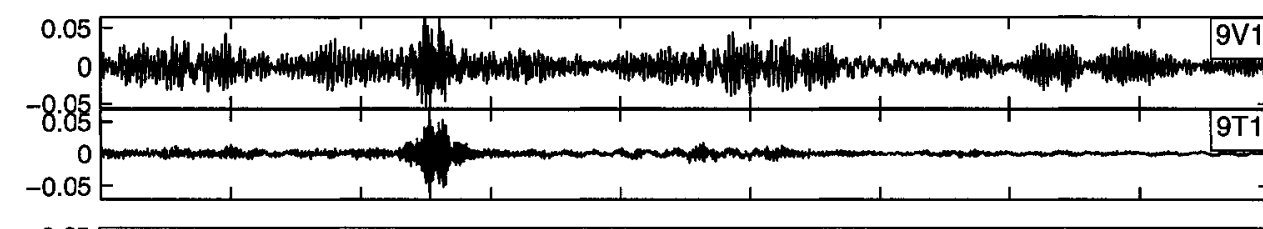

$14 / 12 / 2000$

00:13 AM

Traffic test

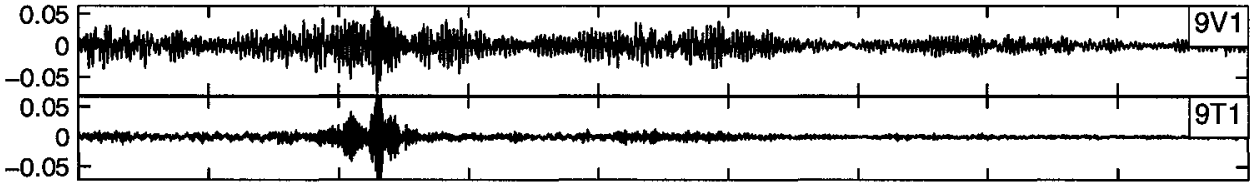

$14 / 12 / 2000$

01:38 AM

Traffic test

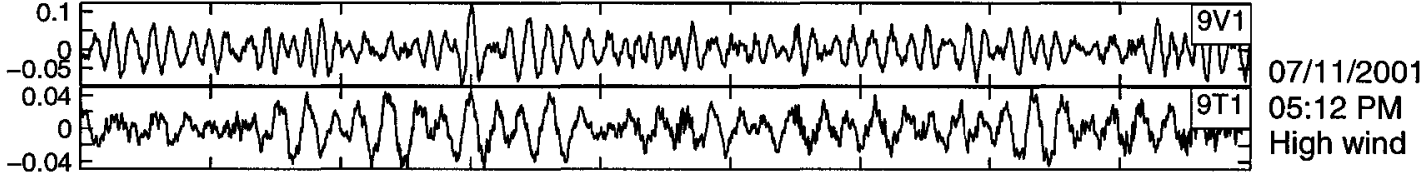

2.02

$22 / 03 / 2002$

06:38 PM

High wind

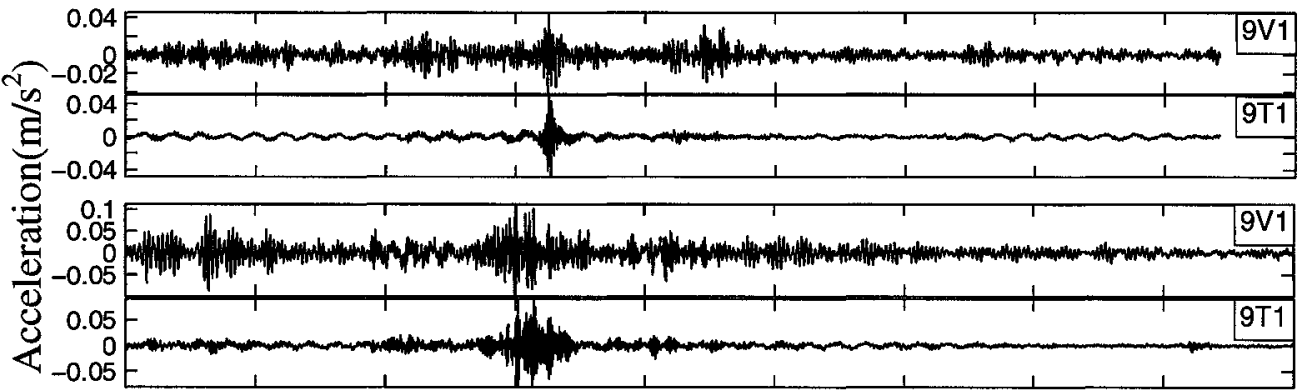

$18 / 02 / 2002$

04:58 AM

Ambient with ice cover

$21 / 02 / 2002$

02:09 PM

Ambient with ice cover

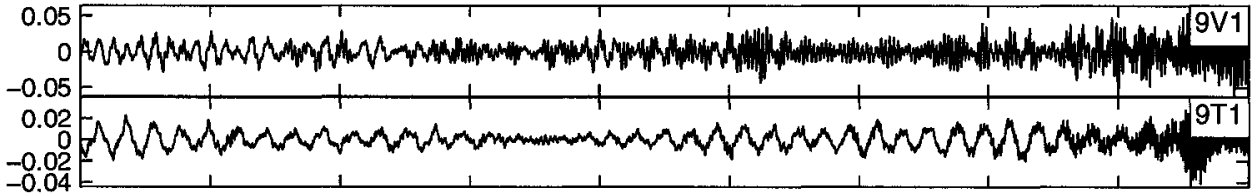

$18 / 11 / 2000$

02:45 PM

Ambient
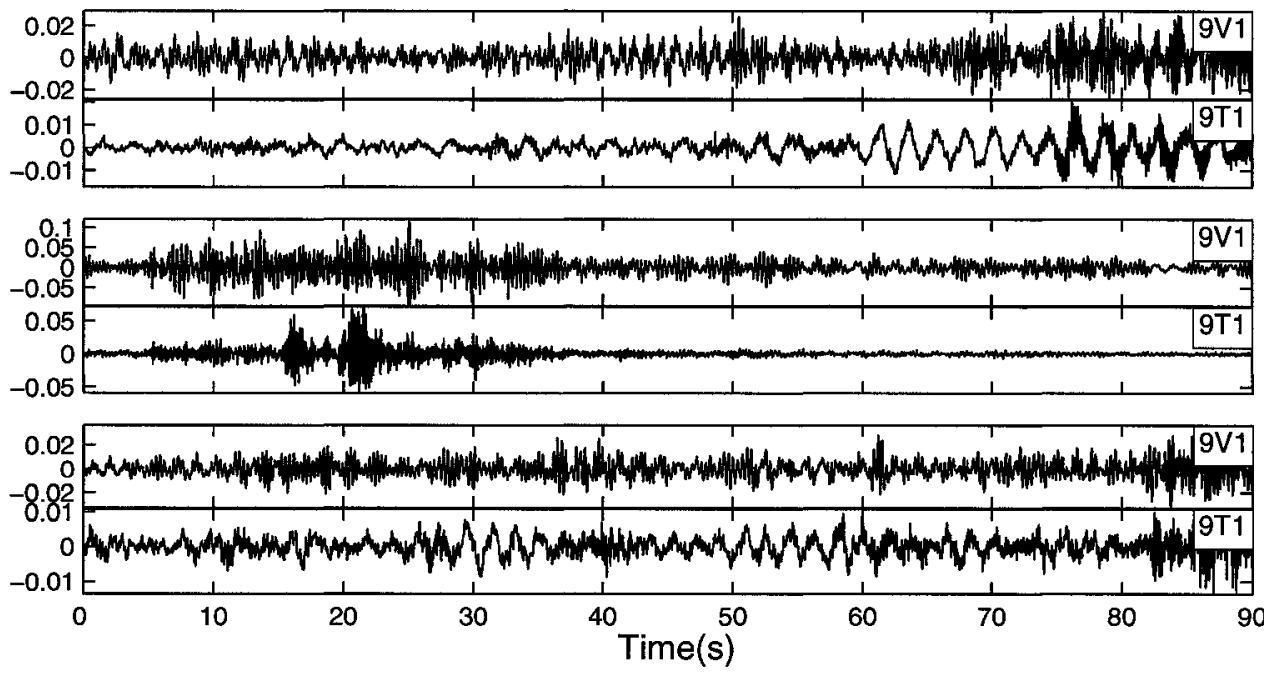

23/05/2002

05:52 PM

Ambient

Figure 7.3. Vertical and transverse acceleration time histories for data sets chosen for indepth verification study by Londoño et al. (2004b). 

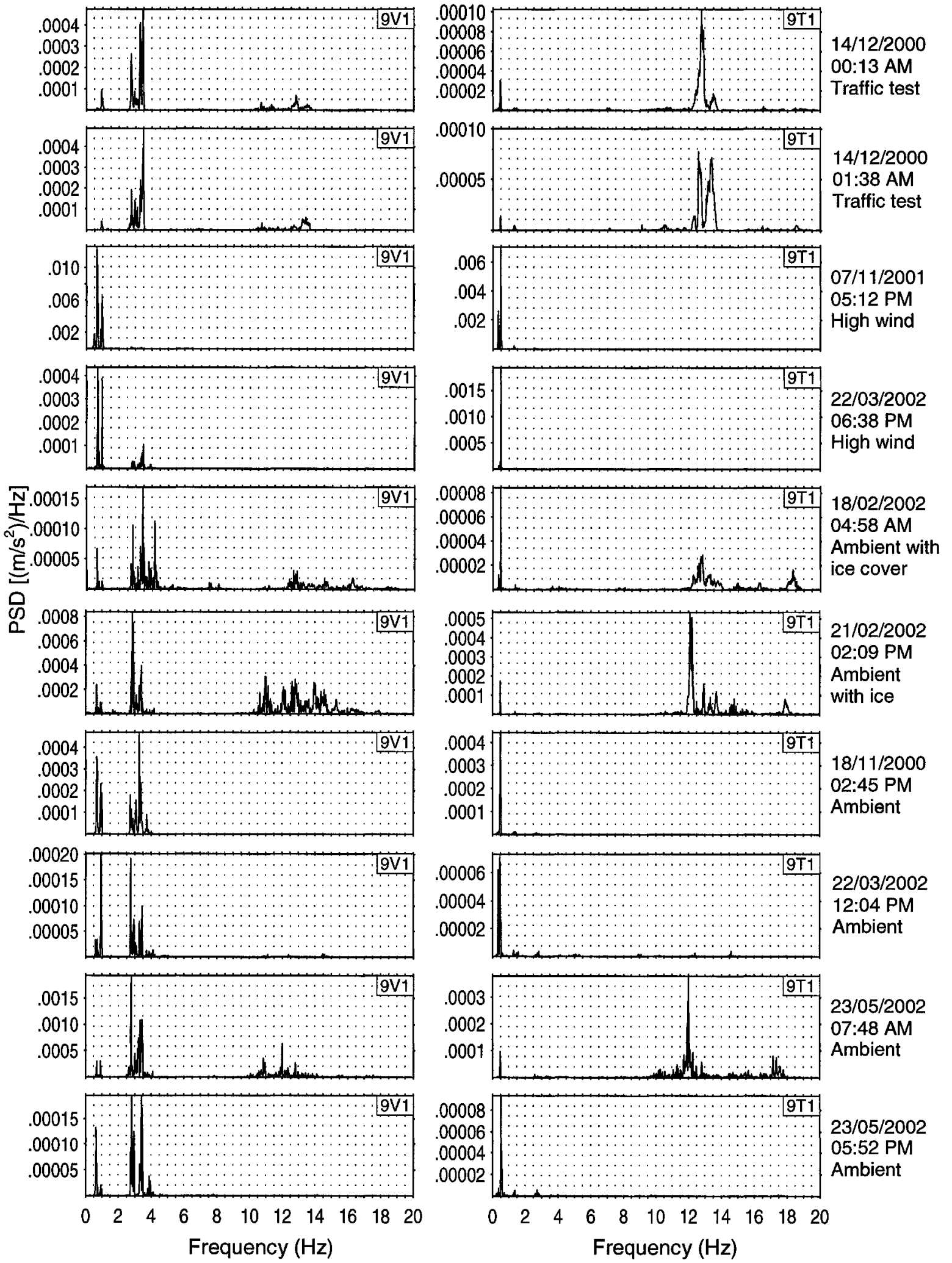

Figure 7.4. Vertical and transverse spectral density estimates for data sets chosen for indepth verification study by Londoño et al. (2004b). 


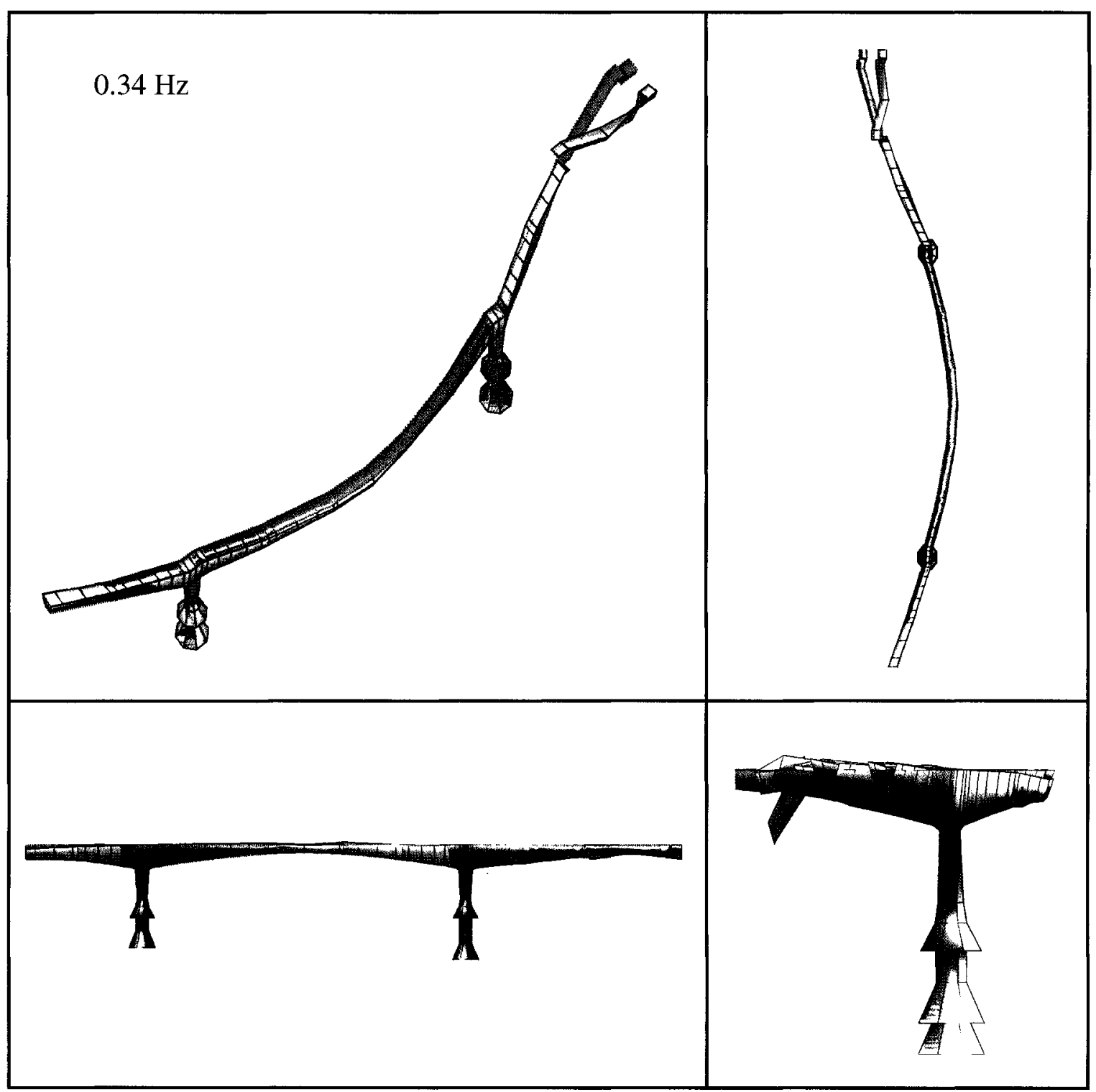

Figure 7.5. Mode-shape comparison of $0.34 \mathrm{~Hz}$ mode. Grey model with full lines represents extracted mode-shapes while green model with dashed lines represents modeshape result from the analytical 3D beam model. 


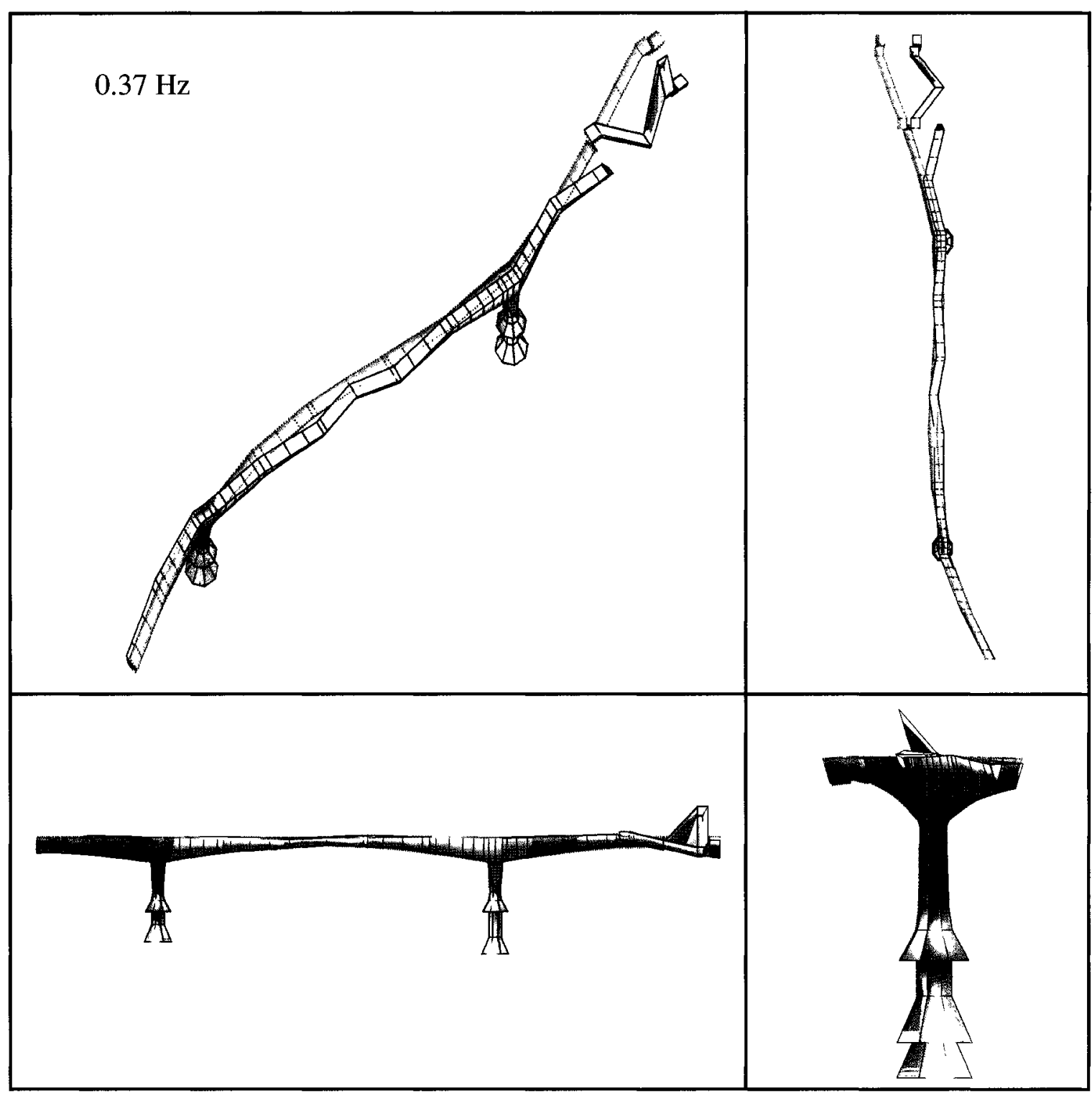

Figure 7.6. Mode-shape comparison of $0.37 \mathrm{~Hz}$ mode. Grey model with full lines represents extracted mode-shapes while green model with dashed lines represents modeshape result from the analytical 3D beam model. 


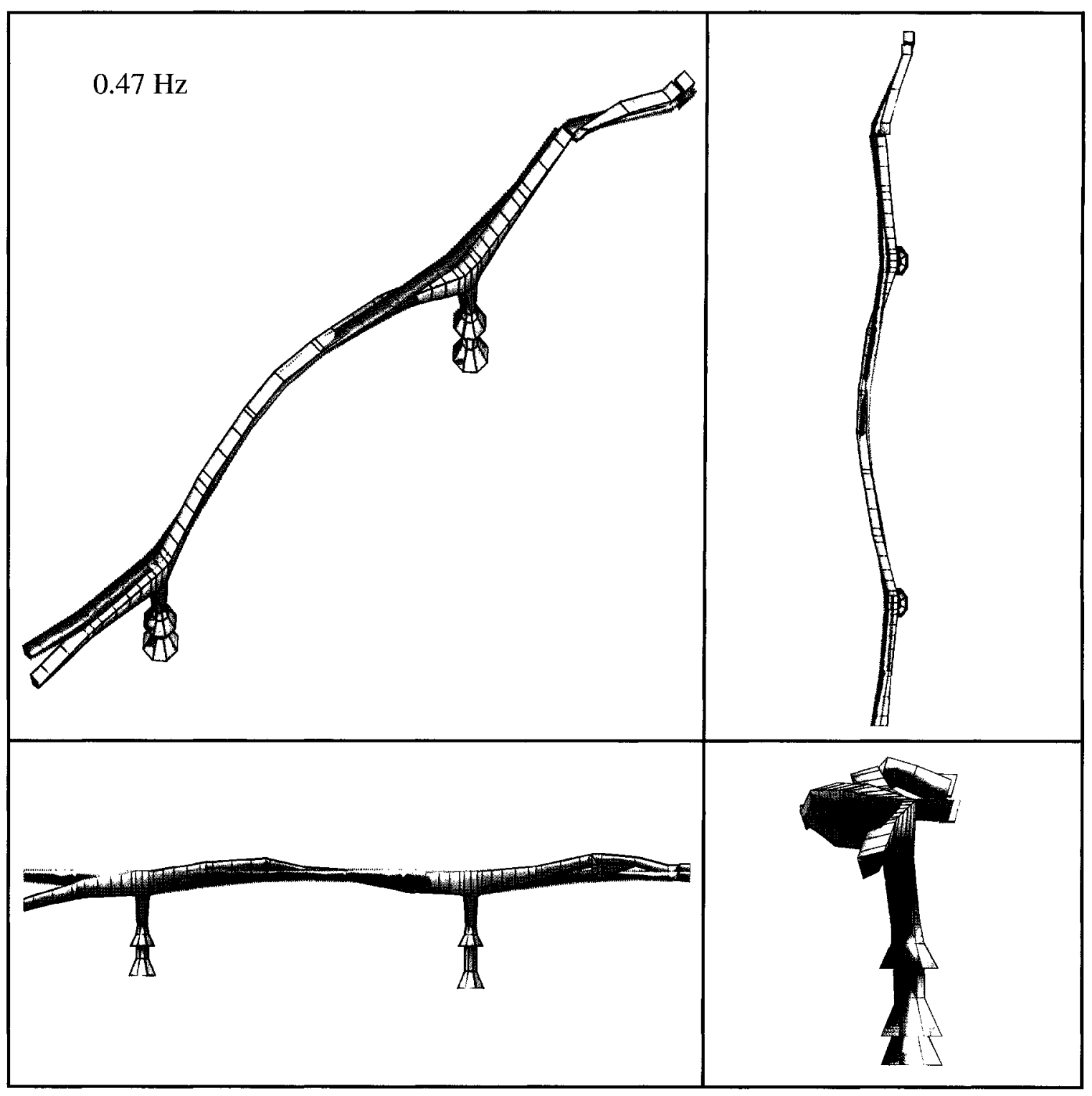

Figure 7.7. Mode-shape comparison of $0.47 \mathrm{~Hz}$ mode. Grey model with full lines represents extracted mode-shapes while green model with dashed lines represents modeshape result from the analytical 3D beam model. 


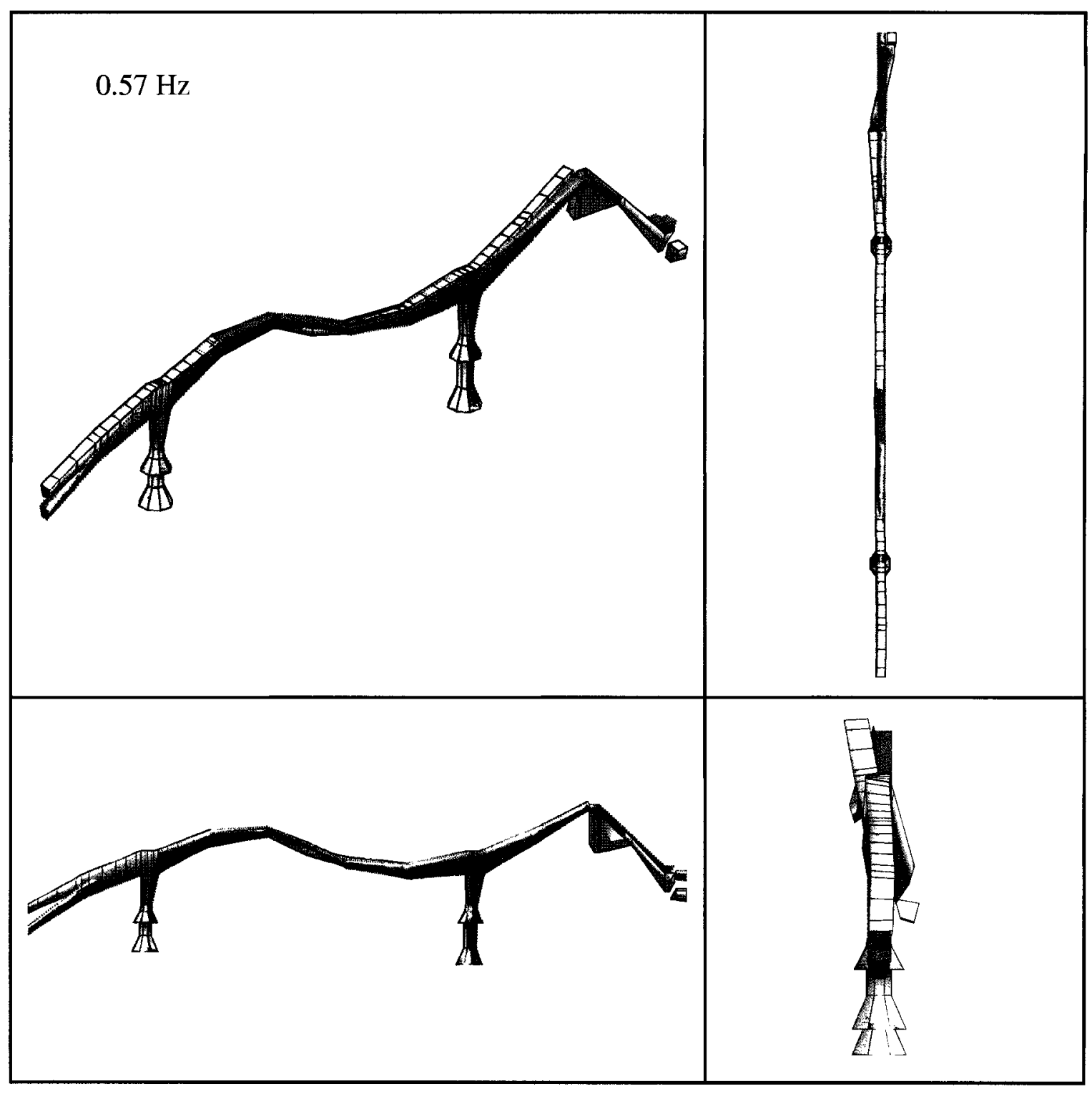

Figure 7.8. Mode-shape comparison of $0.57 \mathrm{~Hz}$ mode. Grey model with full lines represents extracted mode-shapes while green model with dashed lines represents modeshape result from the analytical 3D beam model. 


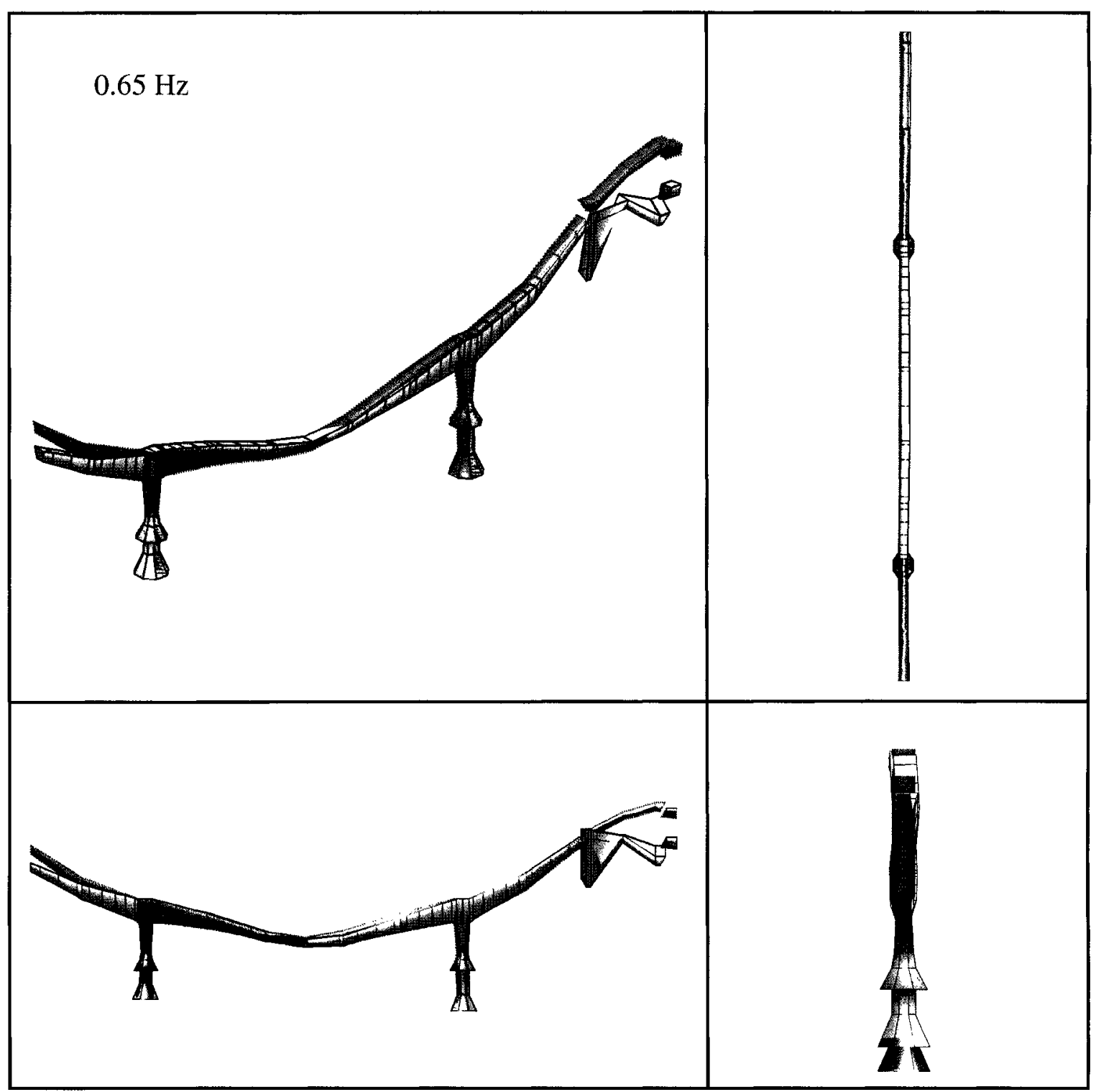

Figure 7.9. Mode-shape comparison of $0.65 \mathrm{~Hz}$ mode. Grey model with full lines represents extracted mode-shapes while green model with dashed lines represents modeshape result from the analytical 3D beam model. 


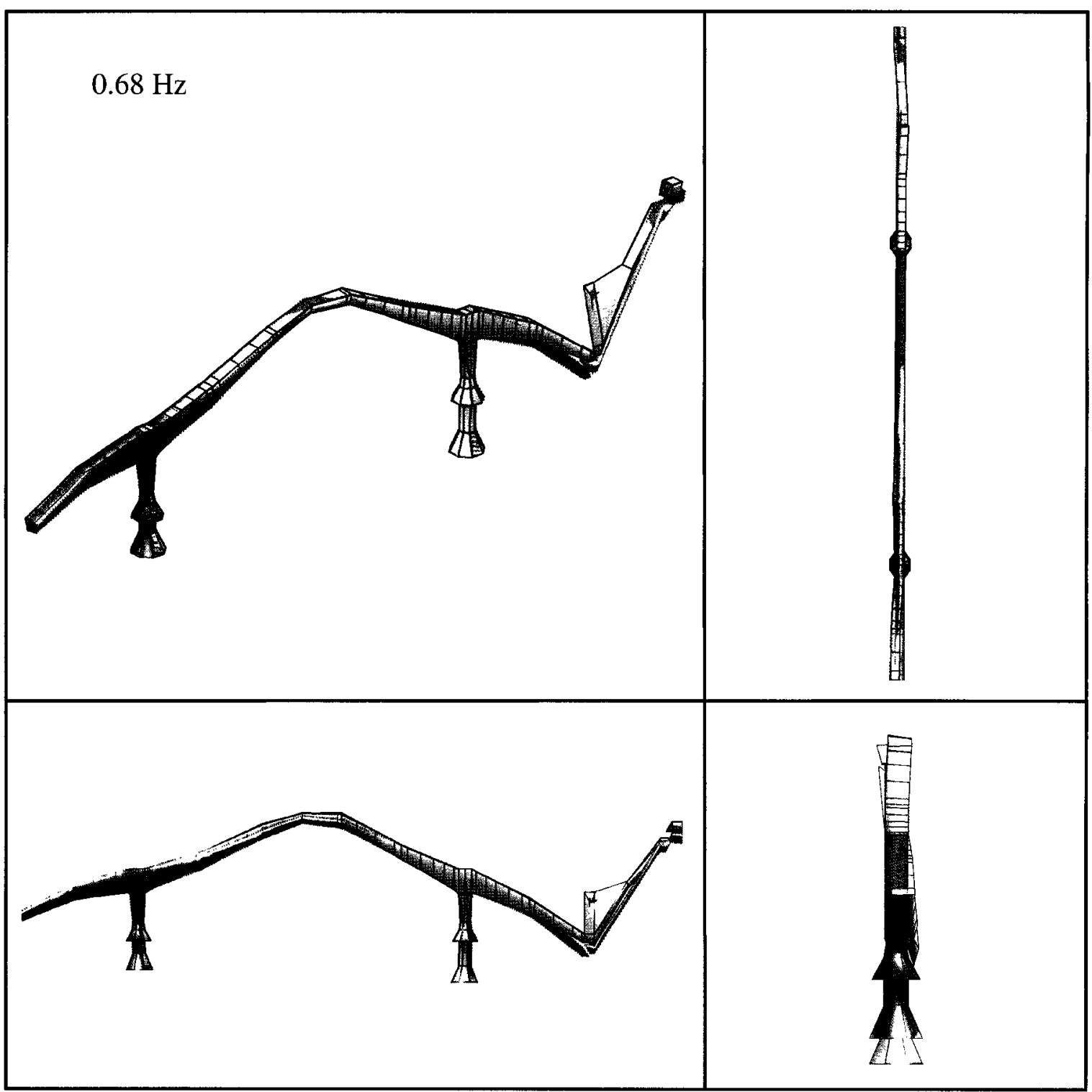

Figure 7.10. Mode-shape comparison of $0.68 \mathrm{~Hz}$ mode. Grey model with full lines represents extracted mode-shapes while green model with dashed lines represents modeshape result from the analytical 3D beam model. 


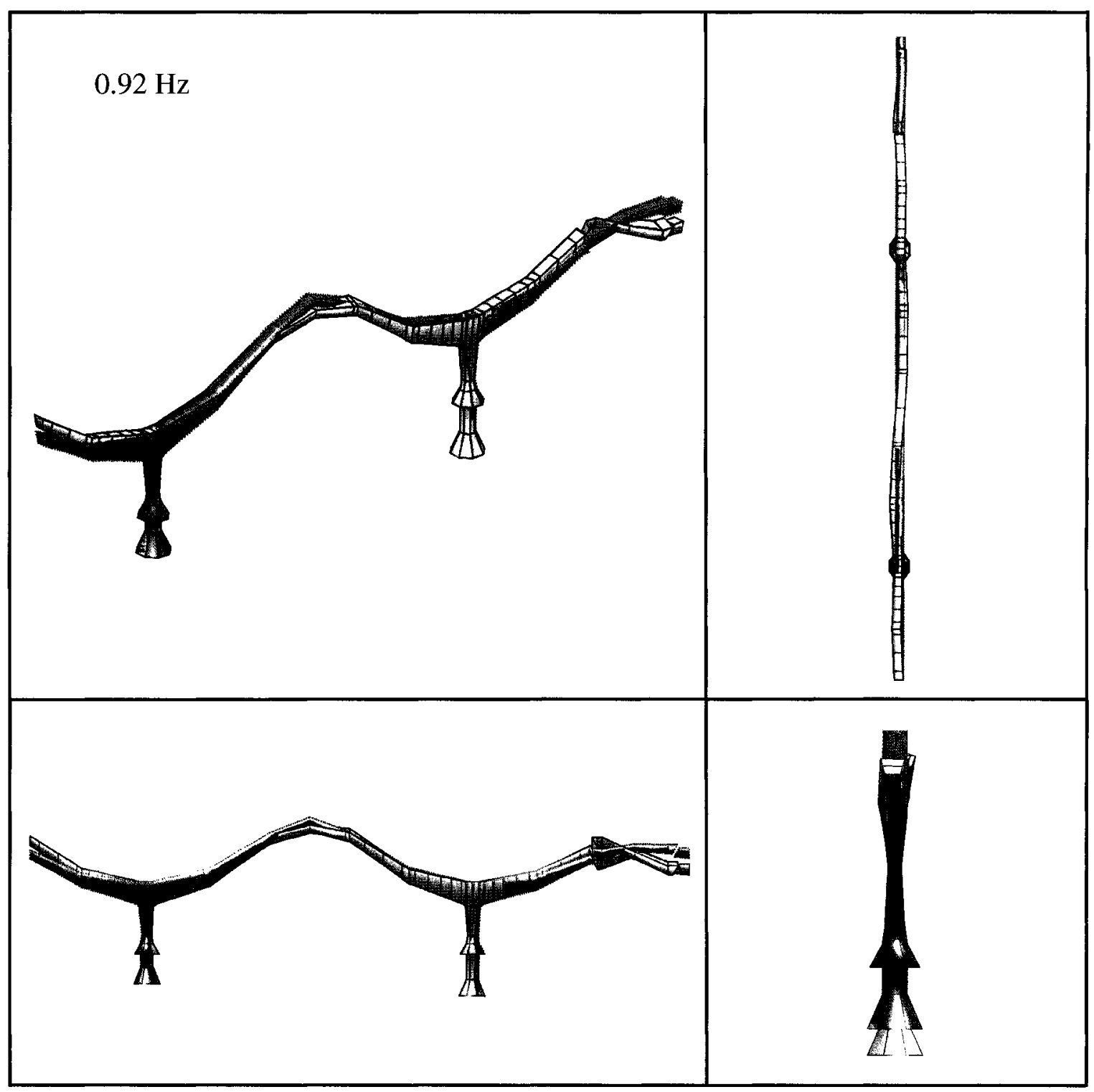

Figure 7.11. Mode-shape comparison of $0.92 \mathrm{~Hz}$ mode. Grey model with full lines represents extracted mode-shapes while green model with dashed lines represents modeshape result from the analytical 3D beam model. 


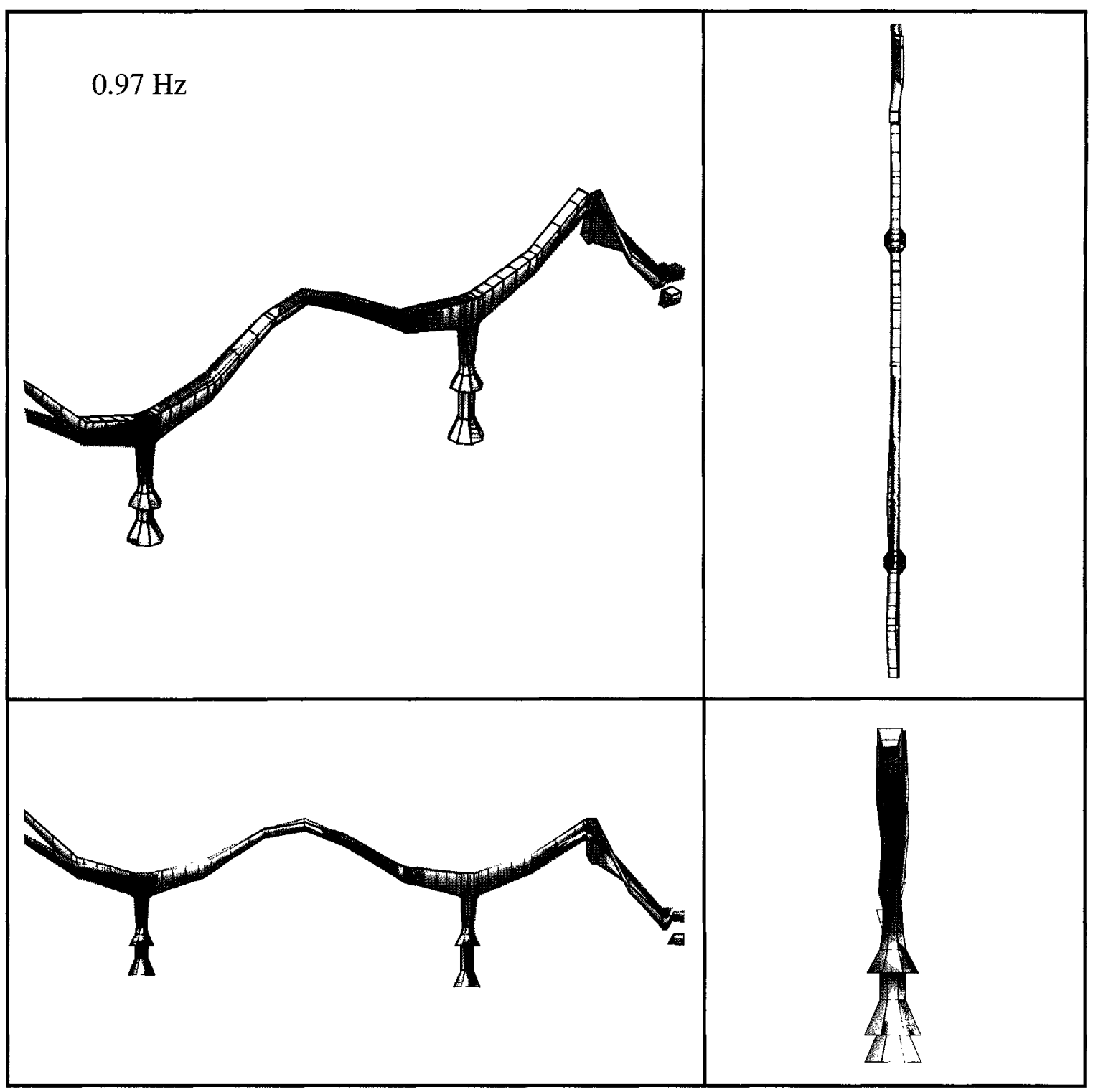

Figure 7.12. Mode-shape comparison of $0.97 \mathrm{~Hz}$ mode. Grey model with full lines represents extracted mode-shapes while green model with dashed lines represents modeshape result from the analytical 3D beam model. 


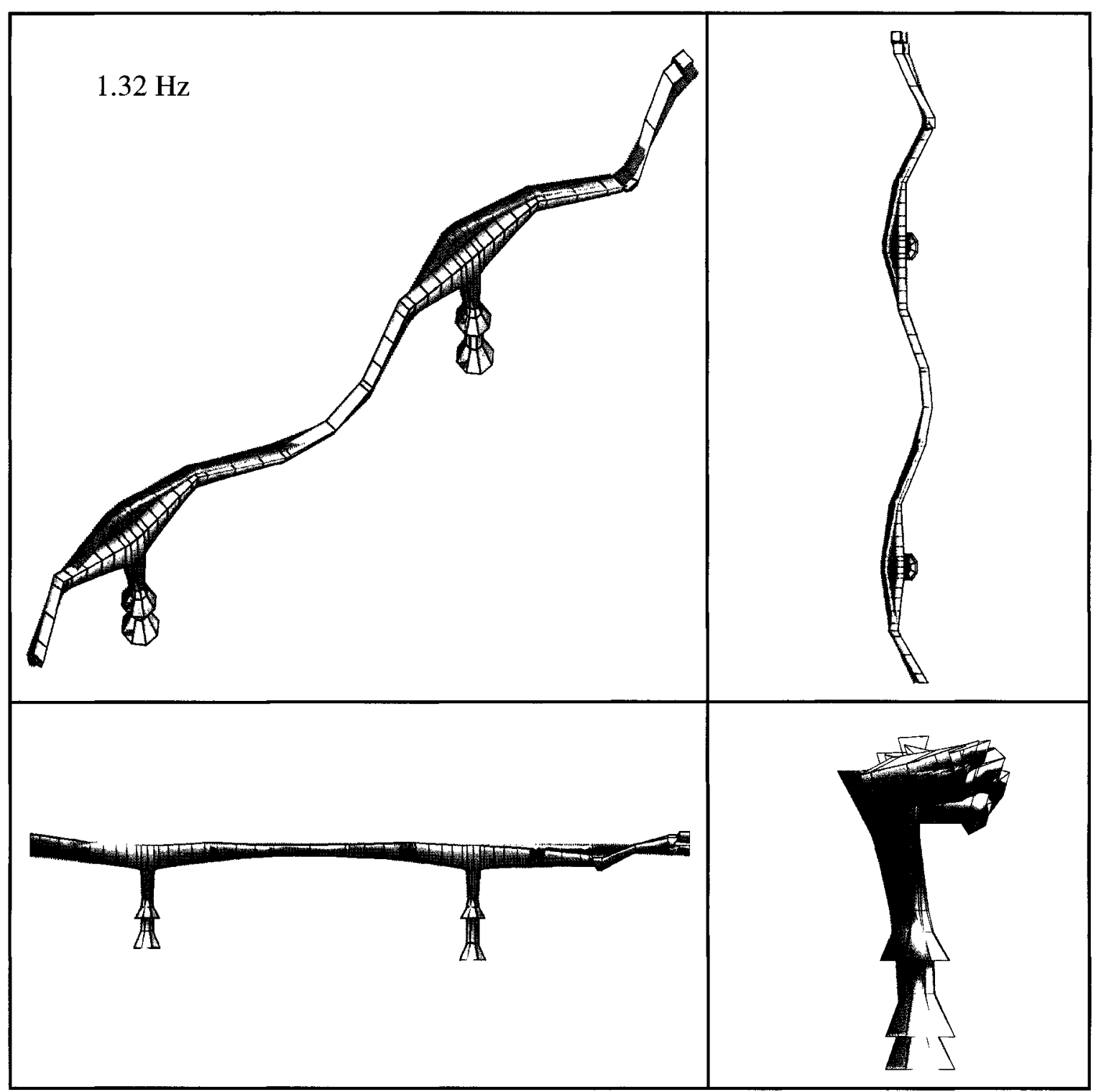

Figure 7.13. Mode-shape comparison of $1.32 \mathrm{~Hz}$ mode. Grey model with full lines represents extracted mode-shapes while green model with dashed lines represents modeshape result from the analytical 3D beam model. 


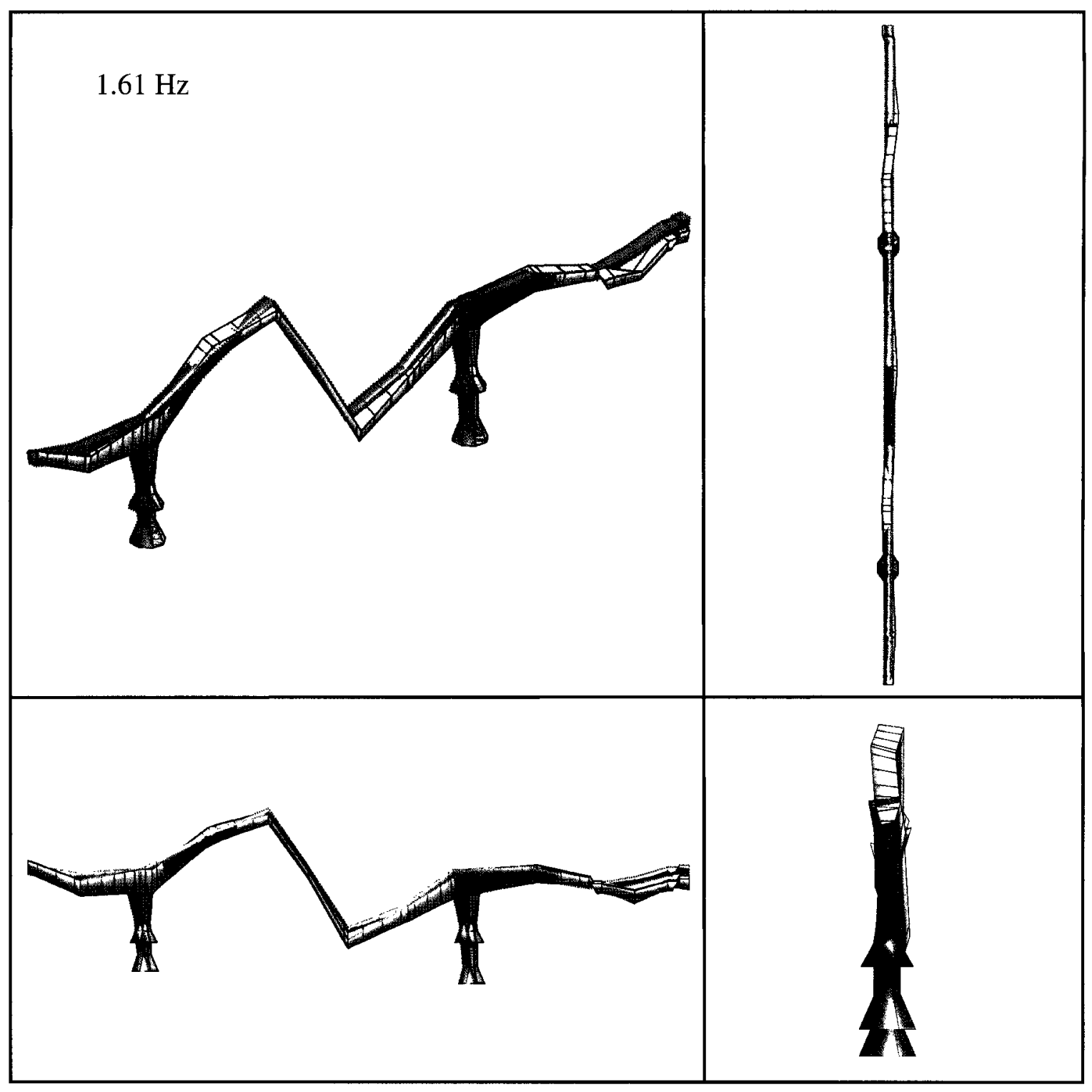

Figure 7.14. Mode-shape comparison of $1.61 \mathrm{~Hz}$ mode. Grey model with full lines represents extracted mode-shapes while green model with dashed lines represents modeshape result from the analytical 3D beam model. 


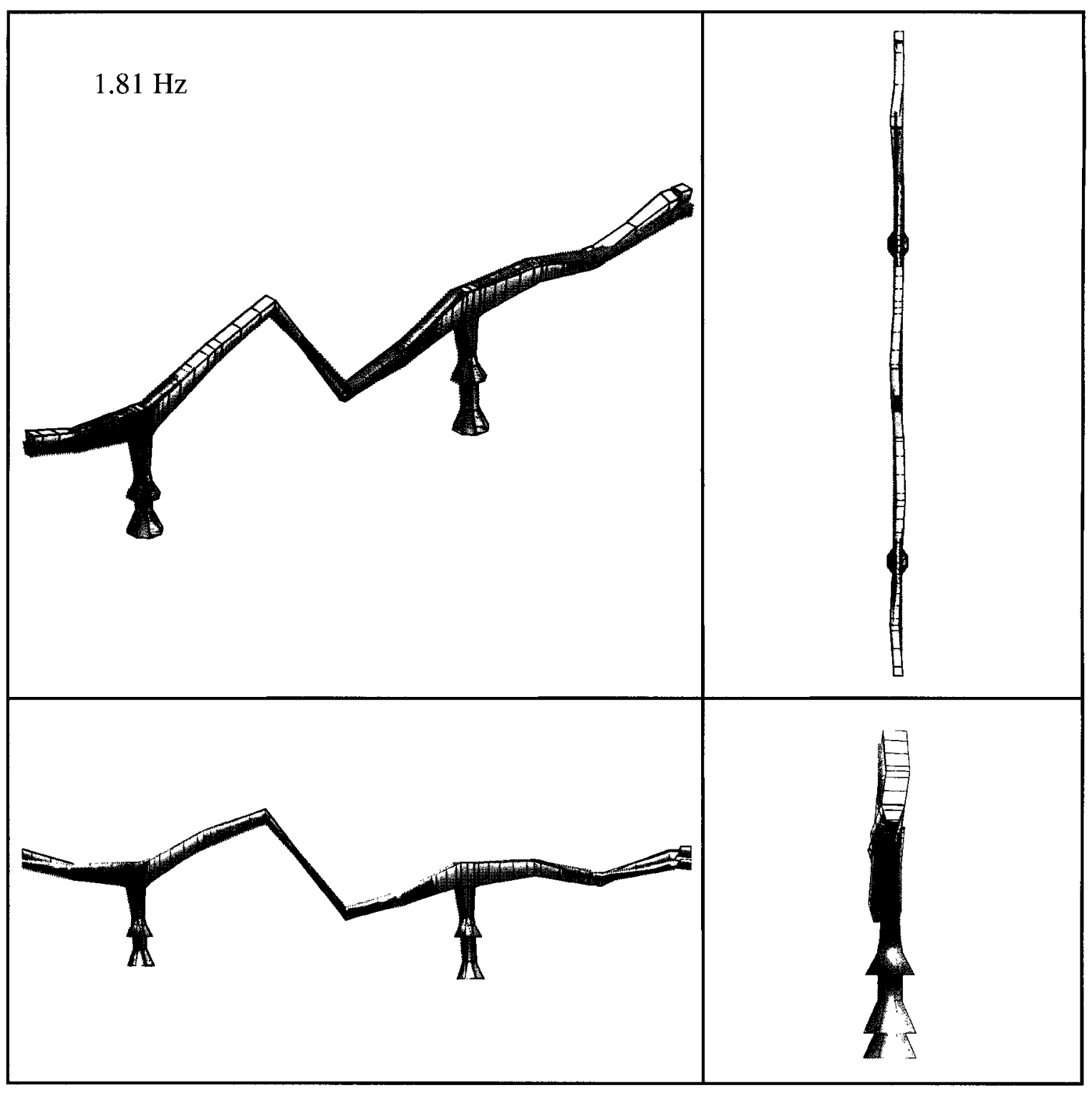

Figure 7.15. Mode-shape comparison of $1.81 \mathrm{~Hz}$ mode. Grey model with full lines represents extracted mode-shapes while green model with dashed lines represents modeshape result from the analytical 3D beam model. 


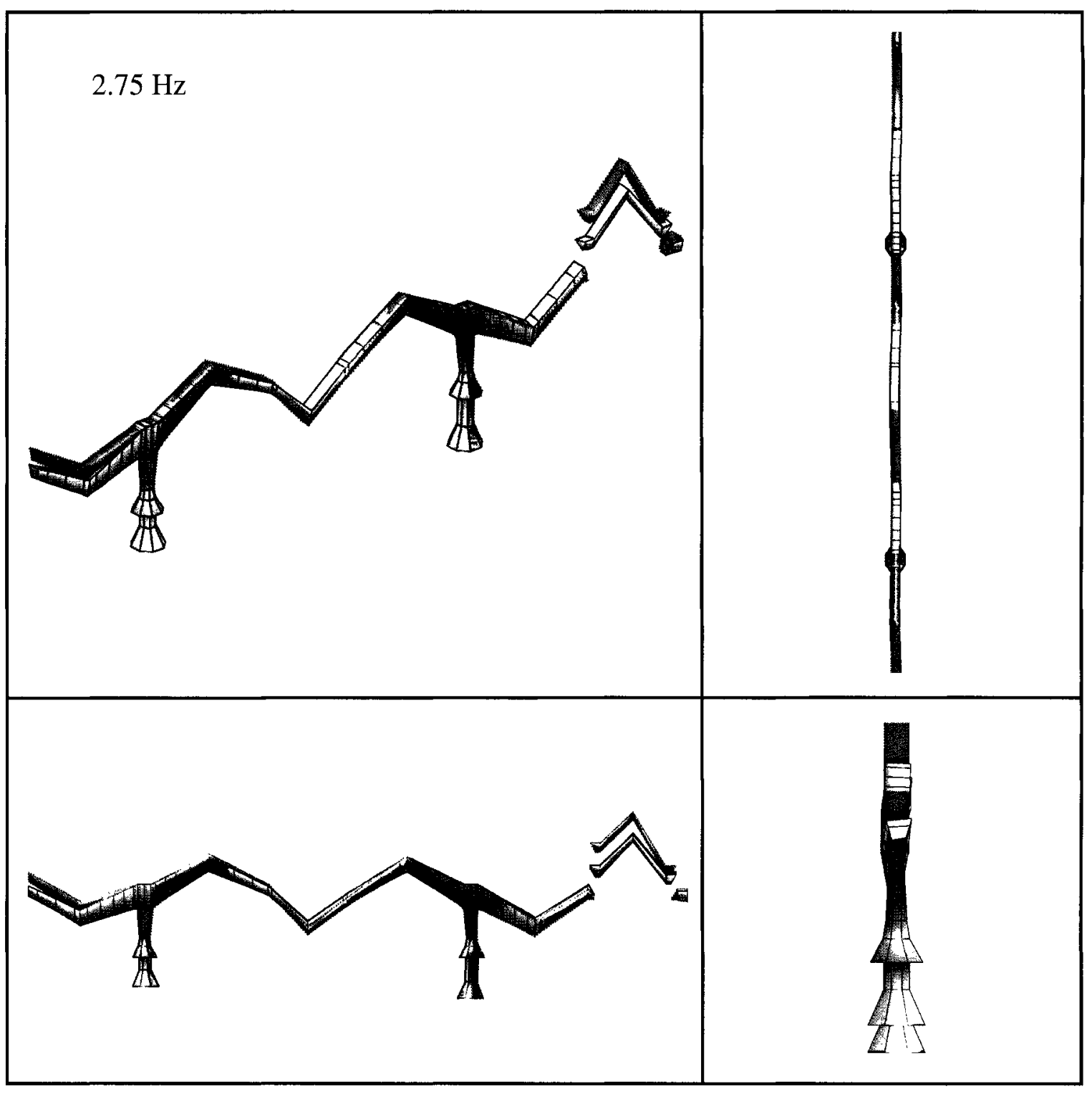

Figure 7.16. Mode-shape comparison of $2.75 \mathrm{~Hz}$ mode. Grey model with full lines represents extracted mode-shapes while green model with dashed lines represents modeshape result from the analytical $3 \mathrm{D}$ beam model. 


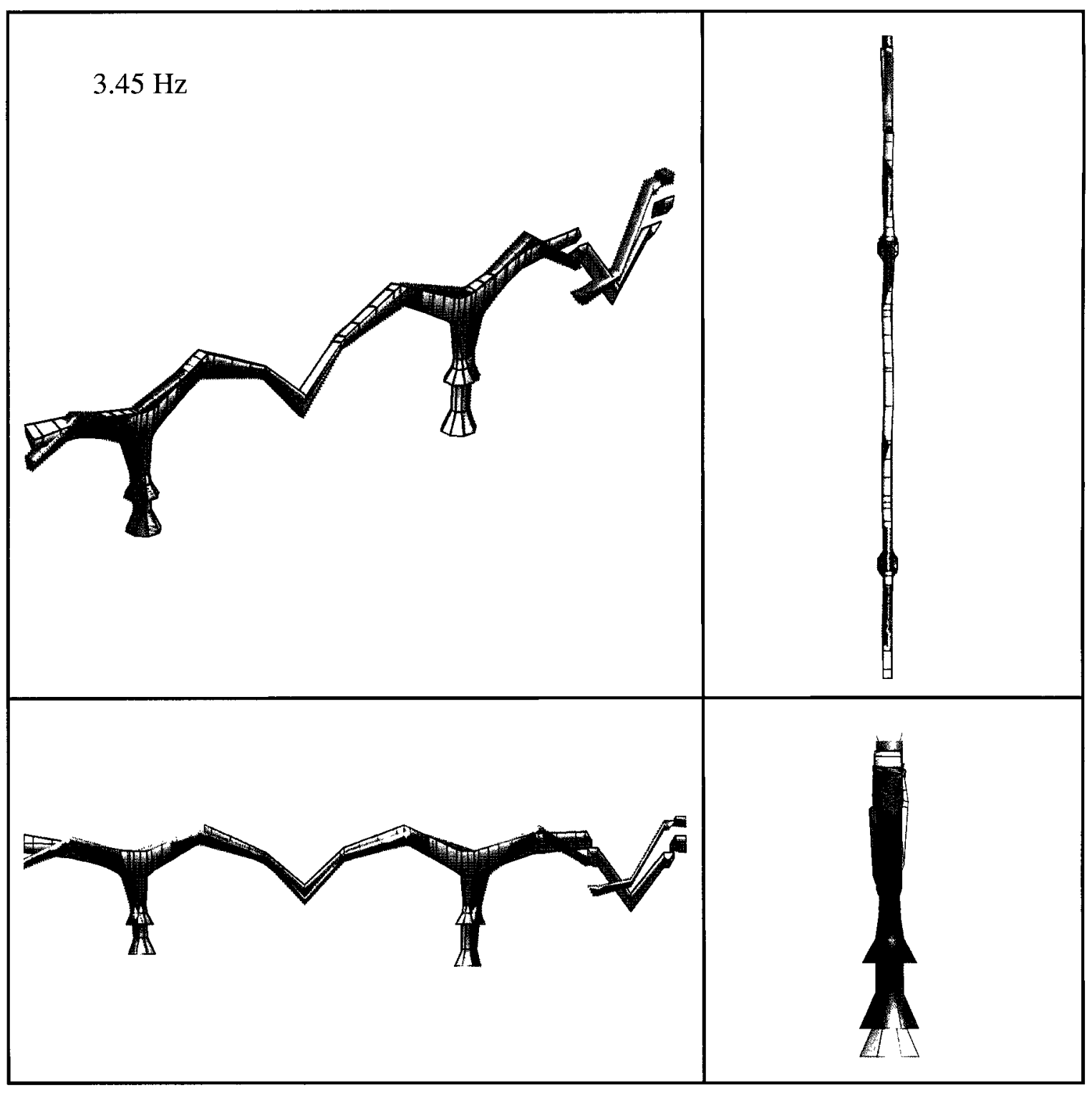

Figure 7.17. Mode-shape comparison of $3.45 \mathrm{~Hz}$ mode. Grey model with full lines represents extracted mode-shapes while green model with dashed lines represents modeshape result from the analytical 3D beam model. 


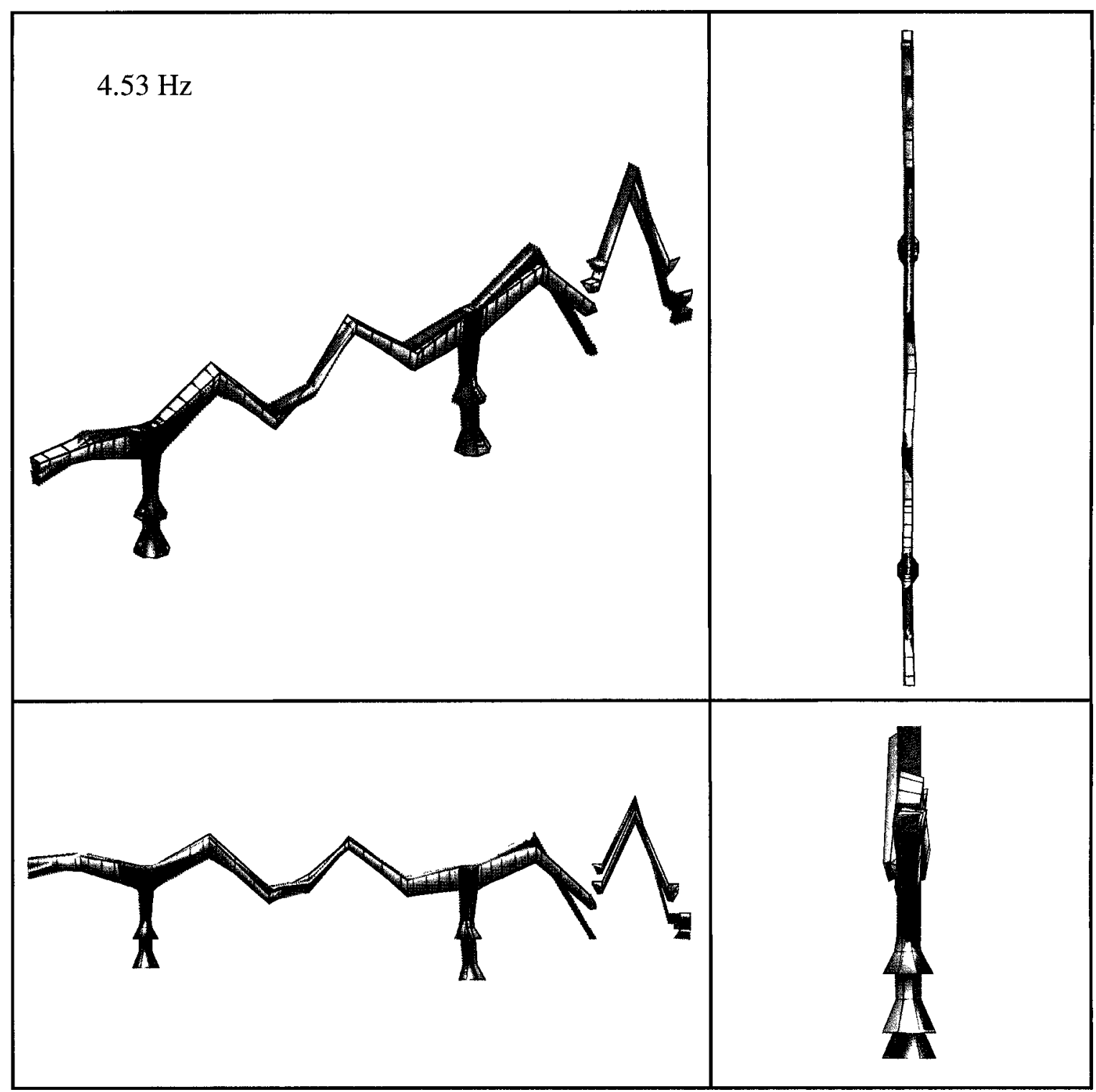

Figure 7.18. Mode-shape comparison of $4.53 \mathrm{~Hz}$ mode. Grey model with full lines represents extracted mode-shapes while green model with dashed lines represents modeshape result from the analytical 3D beam model. 


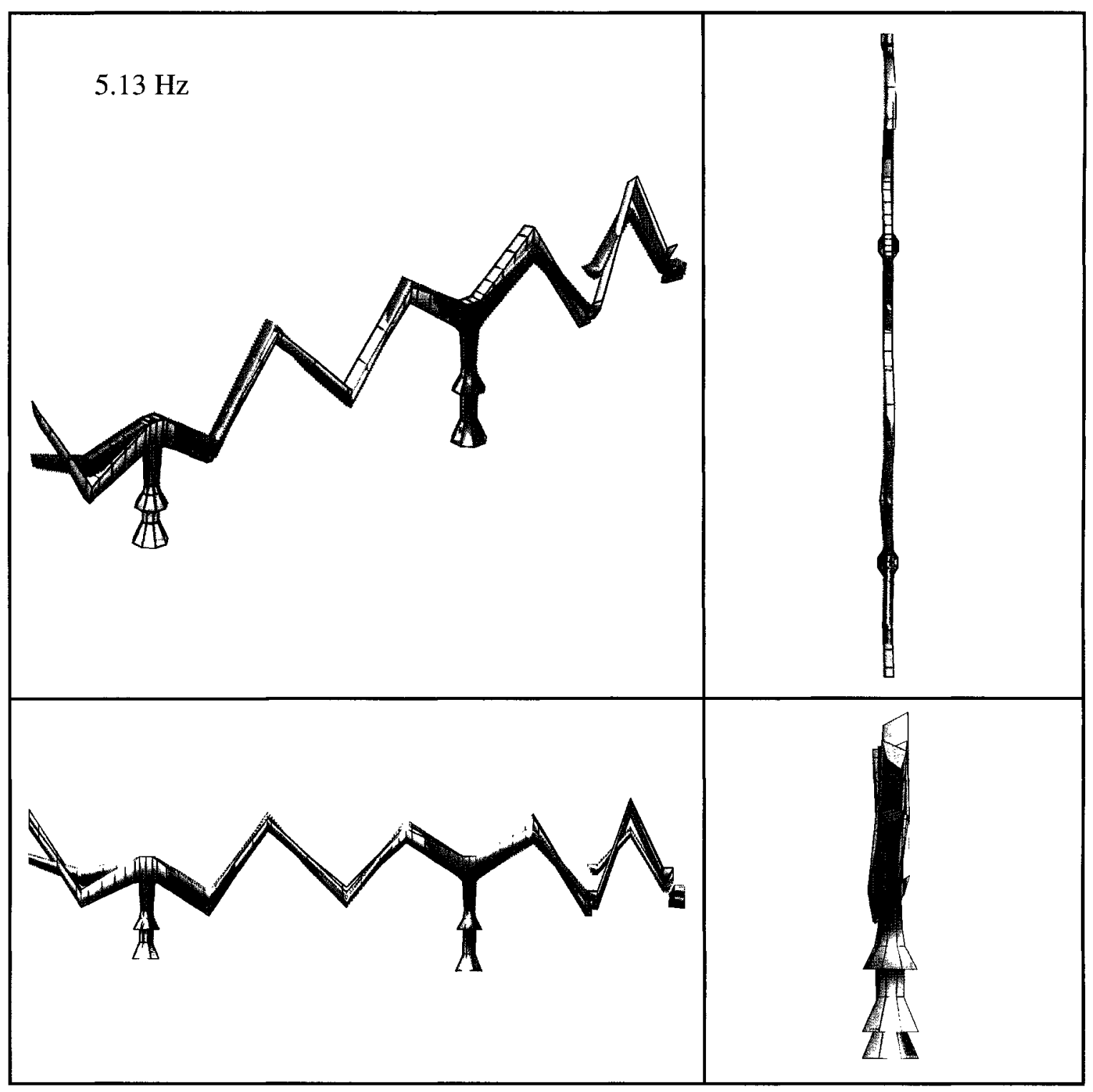

Figure 7.19. Mode-shape comparison of $5.13 \mathrm{~Hz}$ mode. Grey model with full lines represents extracted mode-shapes while green model with dashed lines represents modeshape result from the analytical 3D beam model. 

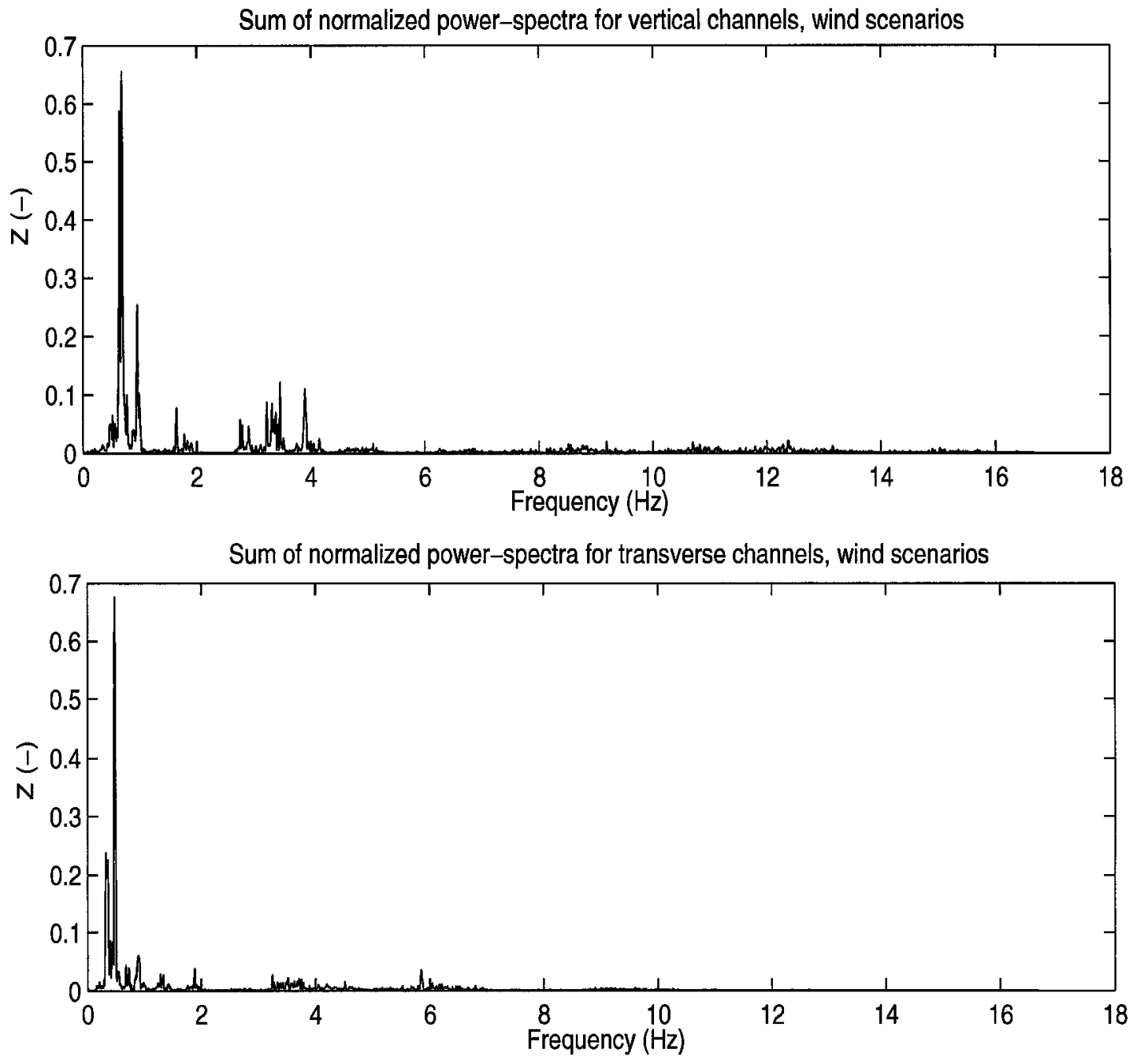

Figure 7.20. Response frequency behaviour to typical high wind scenario. 

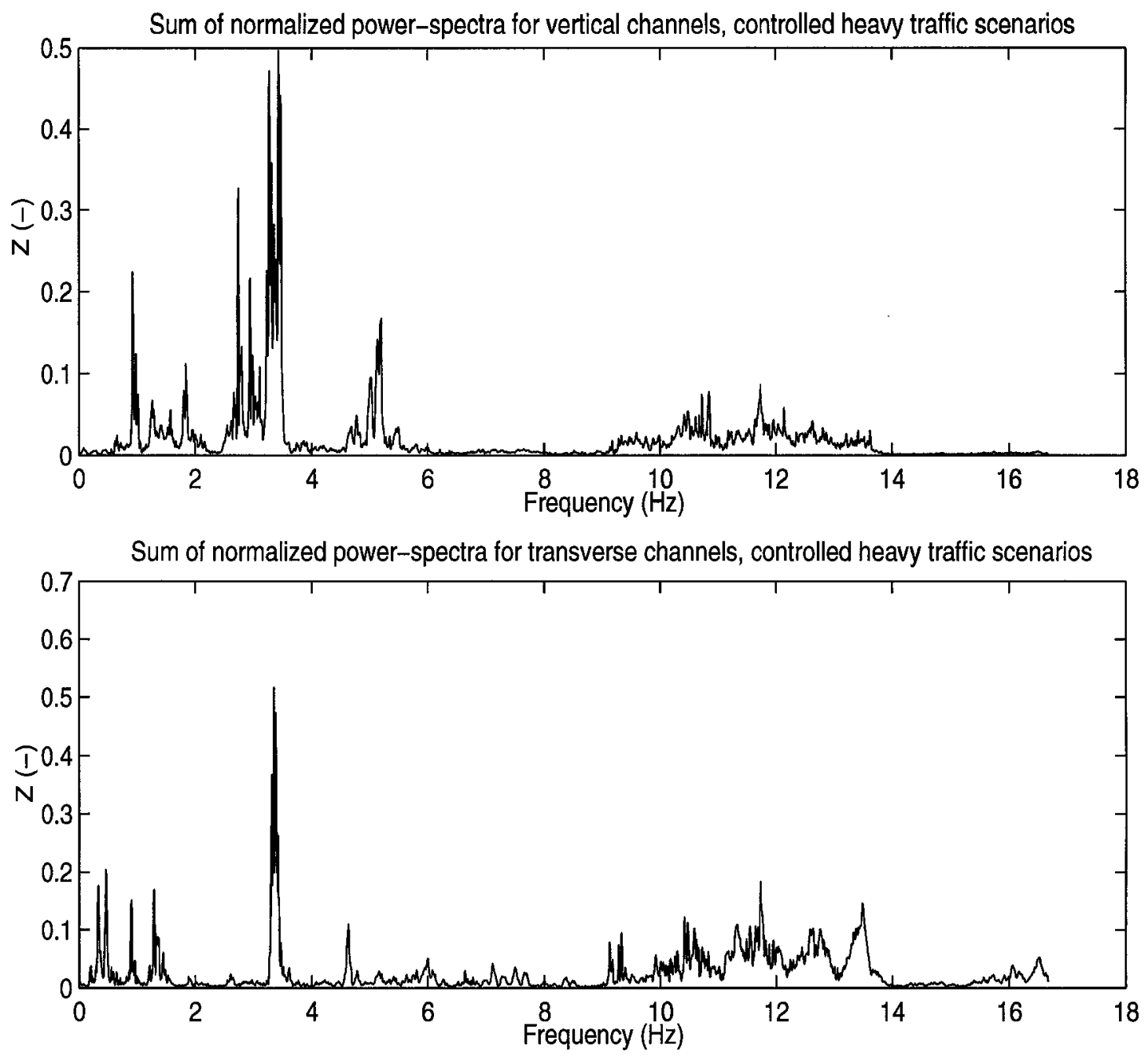

Figure 7.21. Response frequency behaviour to controlled heavy traffic scenarios. 

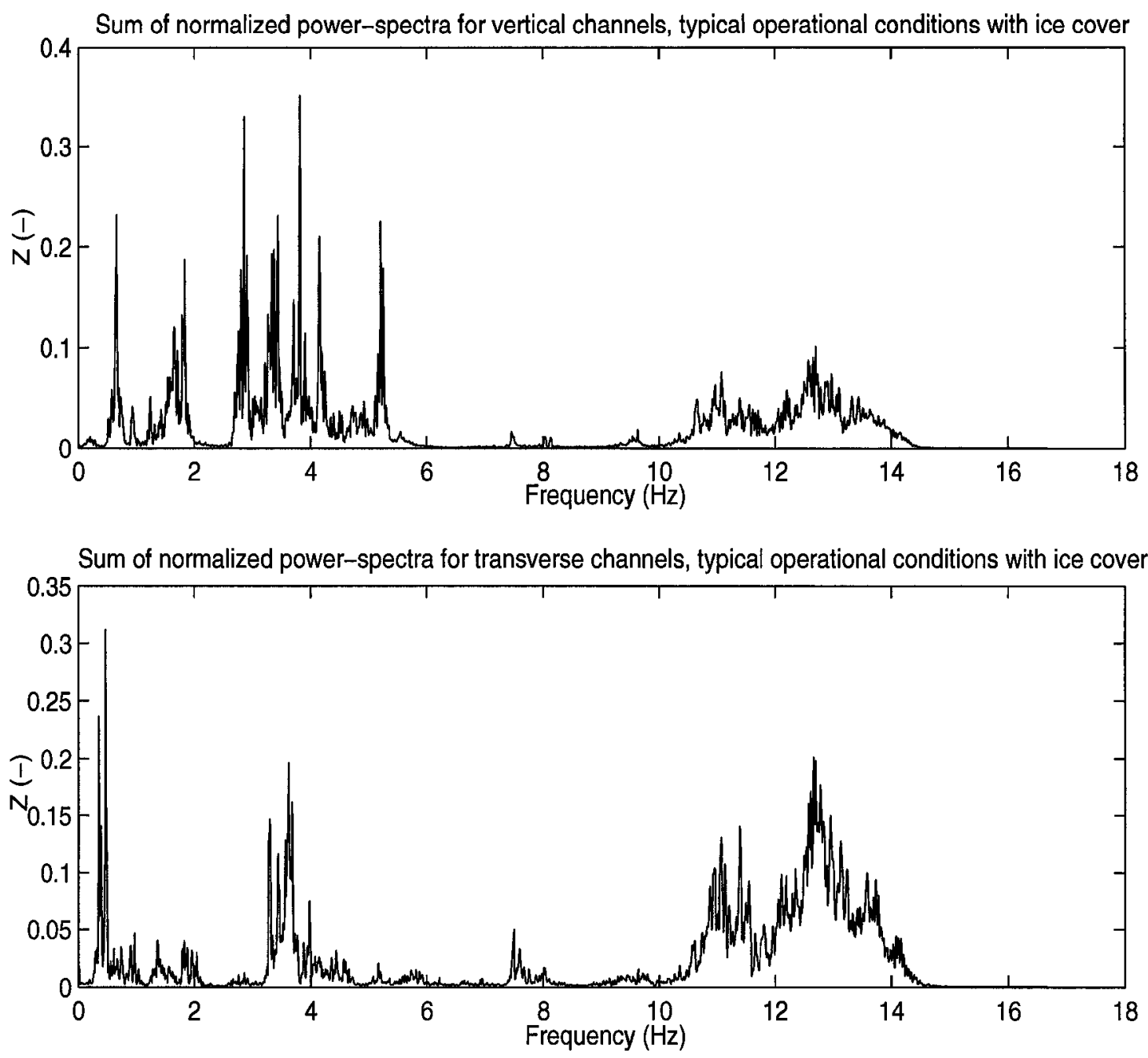

Figure 7.22. Response frequency behaviour to typical ambient scenarios with ice cover. 

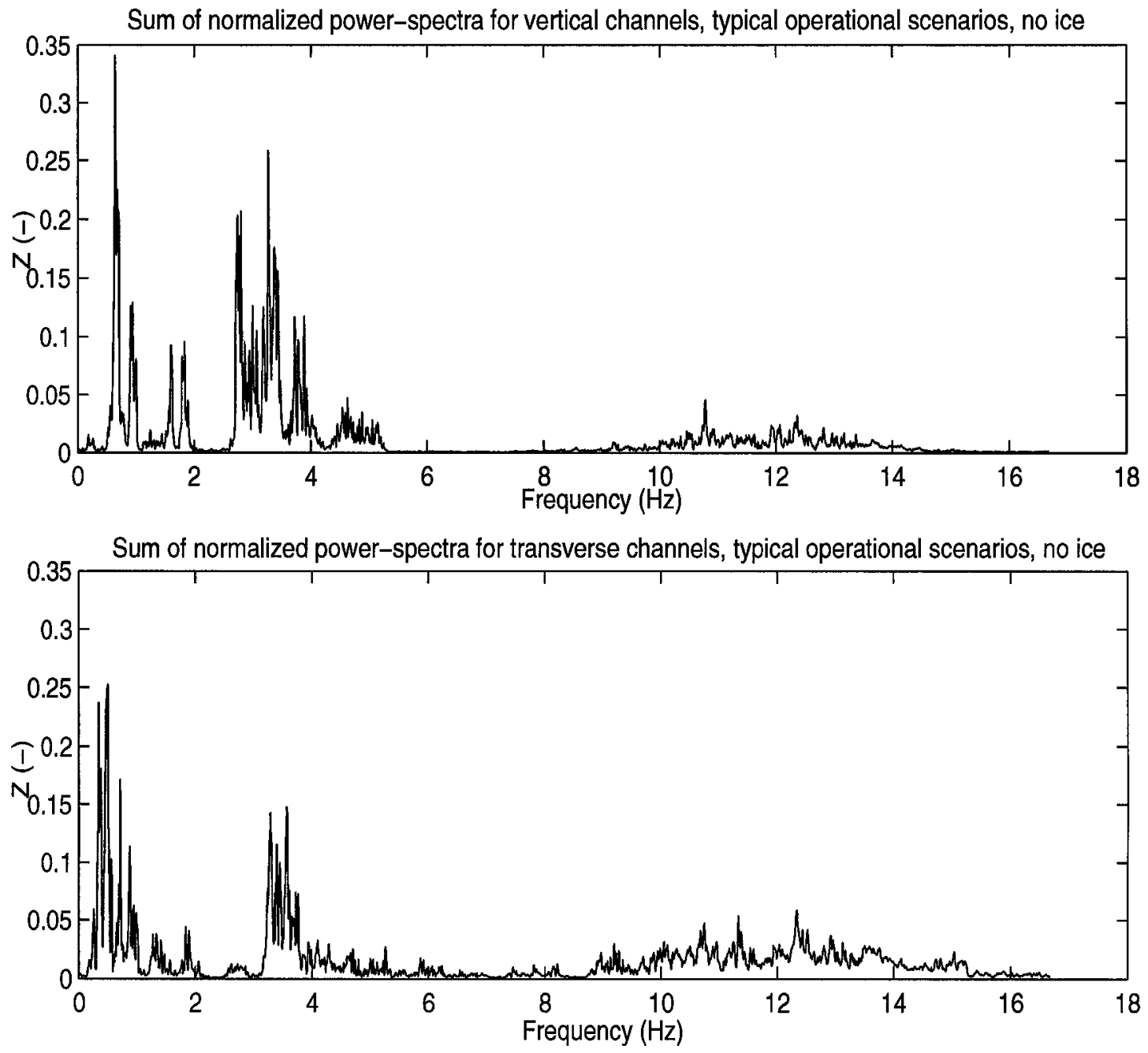

Figure 7.23. Response frequency behaviour to ambient scenarios with no ice cover. 

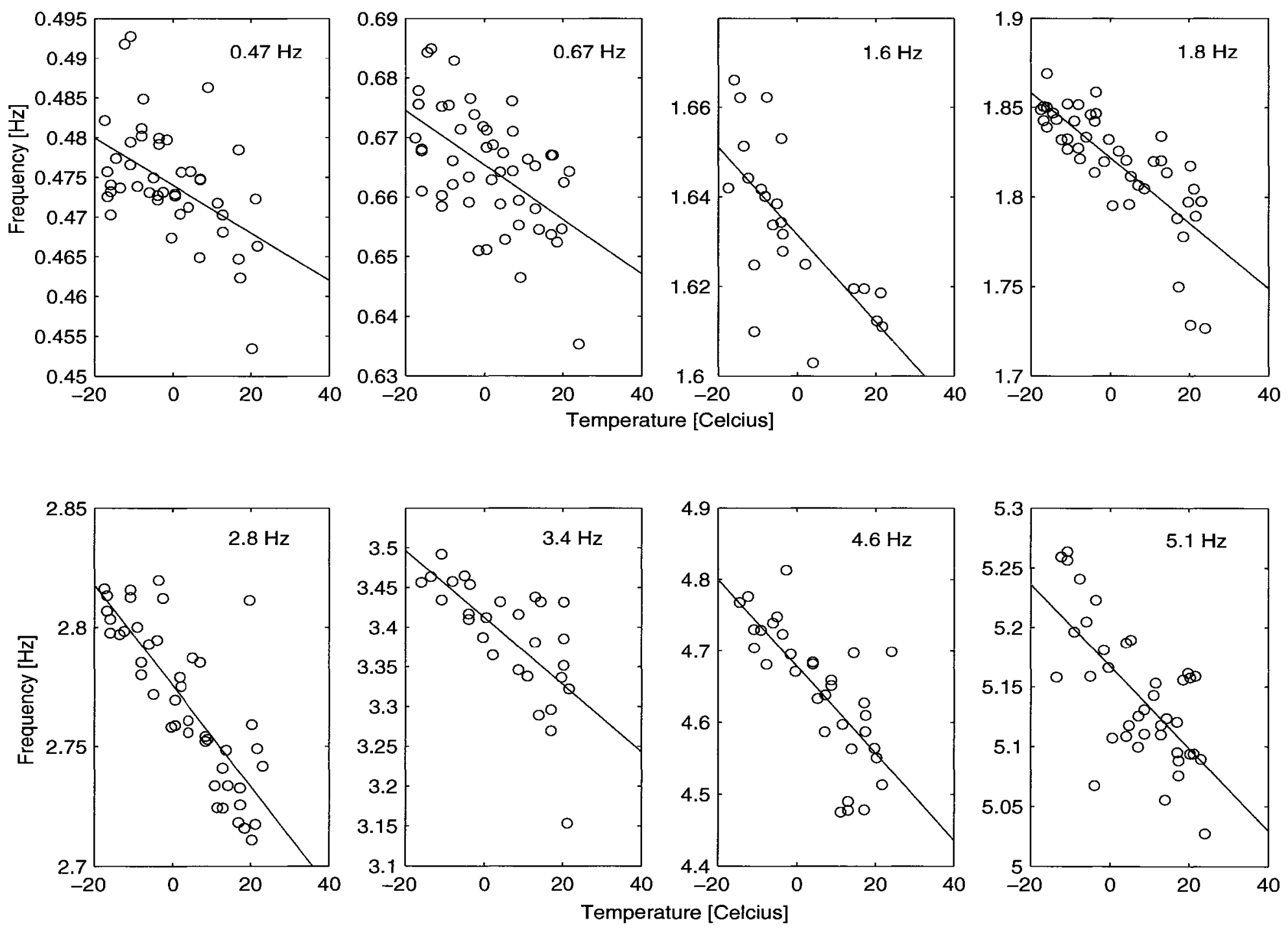

Figure 7.24. Operational modal frequency variation with respect to temperature. 


\section{Chapter 8}

\section{Future Developments and Conclusions}

\subsection{Future Developments and Recommendations}

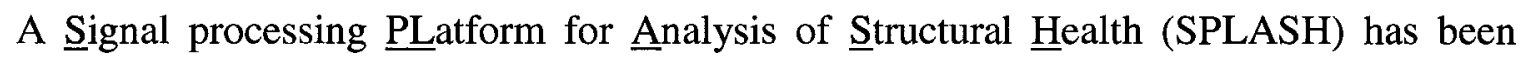
developed for complex data operations including signal processing, system identification analysis and real-time visualization of captured monitoring data and identified structural responses. The SPLASH computer platform has been developed jointly with contributions by N. Londoño. From the experiences of using the developed computer platform and tools in the Confederation Bridge monitoring project, some possible further improvements have been identified. Further developments of SPLASH can be undertaken in the next phase of the Confederation Bridge research program. Summarized 
here are ideas of possible additional developments which can improve and enhance the capabilities and functionality of the developed computer platform.

\subsubsection{Further Research into Variability and Damage Identification}

A brief review of research on the Confederation Bridge monitoring data is given in Chapter 7. Further research may focus on better understanding of the observed variability of the identified modal parameters. It is only after better understanding of the behaviour and characteristics of the monitoring data, especially the factors which influence the variability of the monitoring data, that significant advance can be made in vibration-based structural health monitoring by using the field measurement data to correctly assess the conditions of the monitored structures. After eliminating the contribution to the variability of the monitoring data from environmental factors, any observed deviation may safely be assumed as indication of damage or deterioration. Therefore, detailed studies into causes of variability of dynamic properties will lead to reliable condition assessment algorithms based on field measured deviations of modal parameters which can be correlated to changes of physical conditions of structures. Baseline variability due to the data monitoring and collecting process for the Confederation Bridge monitoring project has been carefully determined to have a standard deviation of $0.6 \%$ of the mean (average of all identified modes included in Table 7.1) which is a promising result that shows it is feasible to use changes in modal properties as an indicator of damage. A general variability of extracted parameters from data collected under varying environmental conditions and loading scenarios has been estimated at about $2 \%$ standard deviation from the mean (averaged over all identified 
modes of Table 7.3). The factors and the sensitivities of these factors contributing to the difference between the two variability levels need to be clearly understood so that any damage corresponding to shifts in dynamic properties exceeding the baseline variability can be reliably detected. Measurement data from environmental effects which are found to contribute to data variability in extracted properties need to be collected as critical part of the monitoring and must be correlated with the extracted dynamic properties to remove its variability effect. The extensive monitoring system currently installed on the Confederation Bridge offers this unique opportunity to assess the influence of various environmental and loading scenarios on the dynamic properties of the structure.

Another important step in the study of variability is to identify the relationship and the sensitivity of the relationship between severity of structural damage and observed deviations in modal properties. This sensitivity study can lead to more critical information on current measurement, processing and analysis techniques, on their adequacy to correctly extract modal properties with sufficient accuracy for detection of potential damage. If it is found that significant damage is masked by normal baseline variability, further enhancement to current measurement, processing and analysis techniques is needed, such as:

- Increasing resolution of data sampling in data acquisition software. Noise created by low resolution sampling of analog signal may contribute to variability of the extracted parameter; studies into the characteristics of noise created by low resolution are currently under way. Improving resolution by dynamic ranging of the sampling operation would be a possible solution to deal with the large amplitude fluctuations of structural responses due to various loading scenarios. 
- Improving current correlation driven SSI technique to employ more reference channels for more accurate, although more computationally intensive, extraction of modal parameters.

- Adopting newer and more computationally intensive numerical SI techniques such as the Hilbert-Huang empirical method which has been shown to deal with nonlinearity and non-stationarity of the structure and its data (Huang et al. 1998, Yang et al. 2003a, 2003b). This method has also been shown to have the advantage of capturing sudden shifts in frequency due to damage which may be lost in averaging operations employed by other SI methods.

For level 2 damage identification of the Confederation Bridge which deals with locating damage on a structure, local vibrations modes observed in the 10 to $16 \mathrm{~Hz}$ range could provide good information. These local vibrations have been observed to affect the contour shape of the box girder cross-section of variable proportions as shown by $\mathrm{Li}$ and others of the monitoring research team. These local vibrations are caused by moving loads such as heavy loaded trucks passing through measured cross-sections at a certain speed. If changes in mode-shapes or frequency are observed at a given cross-section, damage or deterioration may be assumed to be located in the near vicinity of the affected cross-section. An advantage of using higher order modes is the possibility of a more consistent identification of these modes as compared to modes below $1 \mathrm{~Hz}$, as shown in Table 7.3. A study comparable to the one co-authored with Londoño, Lau and Li (Londoño et al. 2004b) and briefly presented in Section 7.2.1 with results in Table 7.3 of this thesis should be considered for modes in the 10 to $16 \mathrm{~Hz}$ range to verify if these modes are more consistently identified than other lower modes. The local vibration 
modes may be a promising area for research in the development of a damage detection algorithm.

\subsubsection{Damage Identification Module}

A robust and efficient damage identification or condition assessment algorithm is the ultimate objective of current research program. Studies undertaken on the variability of dynamic properties contribute to future practical applications of damage identification. Integration of these findings and methodologies into an algorithm capable of correlating environmental data with the response data to remove any effects on the extracted modal properties will lead to a successful condition assessment module for structural health analysis.

\subsubsection{Real-Time Continuous Health Monitoring}

Continuous monitoring requires real-time processing and analysis capabilities of huge amounts of streaming data or data packets which also need to be archived in databases. SPLASH is currently programmed to process data packets incoming from the Confederation Bridge in a real-time manner while the SI module which extracts the much needed modal properties from the data-packets will need modifications to automate the process of identifying stable poles from the stabilization diagram. The recent advances in computer pattern recognition may aid with this automation. 


\subsubsection{Engineering Report Module}

With the above proposed modification to SPLASH as a continuous VBSHM application, a module providing detailed engineering information for structural engineer, operator or end-user is necessary. This module can provide operational information on the structure based on the extracted information from the monitoring data, such as reports of daily maximum and average values of accelerations and deflections, plots of identified modal properties for the day and any significant information which may need attention from bridge operators. The engineering report module will permit the end-user to operate SPLASH with minimal interaction while still getting pertinent information on bridge performance.

\subsection{Conclusion}

A computer application platform for vibration-based structural health monitoring (VBSHM) has been created and presented in this thesis for complex data operations including signal processing, system identification and real-time visualization of structural response monitoring data. The need for such a computer application, considering the sheer amount of vibration data involved in the collection of ambient structural responses, has been shown to be very evident. The ultimate objective for such a platform is to aid in continuing research in the emerging field of VBSHM which will ultimately provide structural engineers, operators or end-users a very powerful structural condition assessment tool for efficient maintenance operations or as a means to remotely evaluate structural performance immediately after occurrence of a major event such as an 
earthquake, hurricane or impact. SPLASH includes efficient and robust processing algorithms, which prepares data for analysis by filtering and treating all signal anomalies, integrated into an automated real-time processing module with a highly sophisticated graphical user interface. The analysis module permits the consistent extraction of structural modal properties by system identification techniques and spectral analysis operations incorporated into highly visual modular GUIs. The animation module permits remote real-time visualization of bridge response on detailed 3D bridge models. Extracted mode-shapes can also be animated on the same 3D bridge models for comparison with other extracted mode-shapes or with analytical mode-shapes imported from commercial FEM software. All captured signals may be plotted in the time and frequency domain in the data display module which is highly flexible and permits rapid signal comparison and visual double checks of the processing operations.

Research on the Confederation Bridge monitoring data has progressed from early processing and system identification methods using FFT computations, power spectrum and band-pass filters to time domain operations on a state space model and finally to sophisticated stochastic subspace identification methods which deals with noisy and uncertain output only data by employing correlation algorithms and data time lags. With the exclusive use of the centralized platform detailed in this thesis as a research tool, there has been observation of a significant correlation between temperature and frequency which represents a significant advance in VBSHM research in that temperature effects must be addressed in any subsequent damage identification algorithm. Other significant studies have also used the platform exclusively and have obtained very important and meaningful results in design verification and in the observation and 
quantification of modal parameter variability. These interesting research results have proved the ability of SPLASH as a robust, efficient and practical VBSHM tool for researchers and operators. 


\section{References}

Abe, M., Fujino, Y., Yanagihara, M., Sato, M. (2000) "Monitoring of Hakucho Suspension Bridge by ambient vibration measurement," Proceedings of the International Society for Optical Engineering SPIE Vol. 3995, p. 237-244.

Brigham, E. O. (1988) “The Fast Fourier Transform and Its Applications," Englewood Cliffs, NJ, Prentice-Hall, Inc., 448 pp.

Bogunović Jakobsen, J. (1995) "Fluctuating Wind Load and Response of a Line-like Engineering Structure with Emphasis on Motion-Induced Wind Forces.” Ph.D. Thesis, Department of Structural Engineering, The Norwegian Institute of Technology, University of Trondheim, Trondheim, Norway.

Cheung, M.S., Tadros, G.S., Brown, T., Dilger, W.H., Ghali, A., Lau, D.T., (1997) "Field Monitoring and Research on Performance of the Confederation Bridge," Canadian Journal of Civil Engineering, 24, 951-962.

Cheung, M.S., Lau D.T., Li W.C., (2002) “Data Acquisition System In Confederation Bridge Monitoring Project," Research Report, Ottawa-Carleton Bridge Research Institute, Carleton University, Ottawa, ON, Canada. 
Cole, H.A. (1973) “On-line Failure Detection and Damping Measurement of Aerospace Structures by Random Decrement Signatures," NASA CR-2205.

Desjardins, S.L., Londoño, N.A., Lau, D.T., Khoo, H.A. 2004. "Real-Time Data Processing and Graphical User Interface (GUI) for Continuous Structural Monitoring," Proceedings of the 18th Australasian Conference on the Mechanics of Structures \& Materials, Perth, Australia.

Doebling, S.W., Farrar, C.R., Prime M.B., Shevitz, D.W. (1996) “Damage Identification and Health Monitoring of Structural and Mechanical Systems from Changes in their Vibration Characteristics: A Literature Review," Los Alamos National Laboratory Report LA-13070-MS, Los Alamos National Laboratory, New Mexico, USA.

Eibeck, P.A., Muramatsu, B., Wang W.L. (2000) “Types of Accelerometers," University of California Berkeley (http://bits.me.berkeley.edu/beam/acc_2b.html).

Ghali, A., Elbadry, M., Megally, S. (2000) "Two-year deflections of the Confederation Bridge," Canadian Journal of Civil Engineering, 27: 1139-1149.

Ghali, A. (1996) "Short and long-term deformation of bridges," Proceedings of the 1996 Canada-Taiwan workshop on medium and long-span bridges, OCBRI.

Hamming, R.W. (1998) Digital Filters. Dover Publications, Mineola N.Y.

Huang, N.E., Zheng, Shen, Z., Long, S.R., Wu, M.C., Shih, H.H., Zheng, Q., Yen, N.-C., Tung, C.C., Liu, H.H., (1998), “The Empirical Mode Decomposition and the Hilbert Spectrum for Nonlinear and Non-Stationary Time Series Analysis," Proceedings of the Royal Society of London. Series A. Mathematical, physical and engineering sciences, 454, 903-995. 
Ibraham, S.R. (1977) "A Method for the Direct Identification of Vibration Parameters from the Free Vibration Response," Shock and Vibration Bulletin Vol. 47.

Jaeger, G.L., Mufti, A.A., Tadros, G., Wong, P. (1997) "Seismic design for the Confederation Bridge," Canadian Journal of Civil Engineering, 24: 922-933.

Juang, J.-N. (1994) Applied System Identification. Prentice Hall, Upper Saddle River, N.J.

Katsuchi, H., Yamada, H., Hata, K., Kusuhara, S. (2003) "Full-scale measurement of vibration of Akashi-Kaikyo Bridge," Proceedings of the first international conference on structural health monitoring and intelligent infrastructure, Tokyo, Japan, Vol. 2, pp. 1059-1065.

King, P., Davenport, A. (1997) "The treatment of wind effects for the Northumberland Strait Crossing," Proceedings of the 1996. Canada-Taiwan Workshop on Medium and Long-Span Bridges, Ottawa, Ontario, pp. 55-73.

Lau, D.T., Brown, T., Cheung, M.S., Li, W.C. (2004) "Dynamic modeling and behavior of the Confederation Bridge," Canadian Journal of Civil Engineering, 31, 379-390.

Li, A.Q., Miao, C.Q., Zhao, L. (2003) "The health monitoring system for the Runyang Yangtse River Bridge," Proceedings of the first international conference on structural health monitoring and intelligent infrastructure, Tokyo, Japan, Vol. 2, pp. 1017-1023.

Londoño, N.A., Lau, D.T., (2003a), "Verification of the Dynamic Properties of the Confederation Bridge," Proceedings of the annual conference of the Canadian Society for Civil Engineering, Moncton, Nouveau-Brunswick, Canada, June 4-7 2003.

Londoño, N.A., Lau, D.T., (2003b), "Variability of dynamic properties from Confederation Bridge Monitoring Data," Proceedings of the first international 
conference on structural health monitoring and intelligent infrastructure, Tokyo, Japan, Vol. 1, pp. 543-550.

Londoño, N.A., Desjardins, S.L., Lau, D.T. (2004a) "Use of Stochastic Subspace Identification Methods for Post-Disaster Condition Assessment of Highway Bridges," Proceedings of 13th World Conference on Earthquake Engineering, Vancouver, B.C., Canada, August 1-6, 2004, Paper No. 2714.

Londoño, N.A., Lau, D.T., Desjardins, S.L., Li, W.C. (2004b) "Characteristics of dynamic monitoring data and observed behaviour of the Confederation Bridge," Submitted to Canadian Journal of Civil Engineering (November 3rd 2004).

MacGregor, J.G., Kennedy, D.J.L., Bartlett, F.M., Chernenko, D., Maes, M.A., Dunaszegi, L. (1997). "Design criteria and load and resistance factors for the Confederation Bridge," Canadian Journal of Civil Engineering, 24: 882-897.

Montreuil, M.A., Lau, D.T., Brown, T.G. (1998), “A Distributed Data Acquisition System for Monitoring the Confederation Bridge." Proceedings of the 44th International Instrumentation Symposium, Reno, Nevada, U.S.A. May 3-7 1998, p 318-333.

Naumoski, N., Cheung, M.S., Foo, S. (2002) "Dynamic characteristics of the Confederation Bridge," Canadian Journal of Civil Engineering, 29, 448-458.

Naumoski, N., Cheung, M.S., Foo, S. (2004) "Dynamic performance of the Confederation Bridge due to traffic and wind," Canadian Journal of Civil Engineering, 31, 487-498. 
Peeters, B. (2000) "System Identification and Damage Detection in Civil Engineering." $\mathrm{PhD}$ thesis, Department of Civil Engineering, Katholieke Universiteit Leuven, Belgium.

Peeters, B., Couvreur, G., Razinkov,O., Kundig, C., Van der Auweraer, H., De Roeck, G., (2003), "Continuous Monitoring of the Oresund Bridge : System and Data Analysis," Proceedings of IMAC XXI, A conference and exposition on structural dynamics, Kissimmee, Florida, U.S.A., February 3-6, 2003.

Peeters, B., De Roeck, G., (1999), "Reference Based Stochastic Subspace Identification For Output-Only Modal Analysis," Mechanical Systems and Signal Processing, 13(6), 855-878.

Peeters, B., De Roeck G., Caetano E., Cunha, A., (2002) "Dynamic Study of the Vasco da Gama Bridge," ISMA 2002 International Conference on Noise and Vibration Engineering, Katholieke Universiteit Leuven, Leuven, Belgium.

Welch, P.D. (1967) "The Use of Fast Fourier Transform for the Estimation of Power Spectra: A Method Based on Time Averaging Over Short, Modified Periodograms," IEEE Trans. Audio Electroacoustics, Vol. AU-15 (June 1967), pp. 70-73.

Yang, J.N., Lei, Y., Pan, S., Huang, N. (2003) "System identification of linear structures based on Hilbert-Huang spectral analysis. Part 1 : Normal modes," Journal of Earthquake Engineering and Structural Dynamics, 32, 1443-1467.

Yang, J.N., Lei, Y., Pan, S., Huang, N. (2003) "System identification of linear structures based on Hilbert-Huang spectral analysis. Part 2 : Complex modes," Journal of Earthquake Engineering and Structural Dynamics, 32, 1533-1554. 
Zhang, M. (2002) "System Identification Analysis of the Dynamic Monitoring Data of the Confederation Bridge.” M.A.Sc. thesis, Department of Civil and Environmental Engineering, Carleton University, Ottawa, On.,Canada 


\section{Appendix A - Types of Accelerometers}

Piezoelectric: Piezoelectric transducers are often used in vibration-sensing accelerometers. The piezoelectric crystals, often quartz or ceramic, produce an electric charge when a force is exerted by the vibrating mass under acceleration. The quartz plates are preloaded so that a positive or negative change due to the applied force on the crystals results in a change in the electric charge. Although the sensitivity of piezoelectric accelerometers is relatively low compared with other types of accelerometers, they have the highest range (up to $100,000 \mathrm{~g}$ ) and frequency response (over $20 \mathrm{kHz}$ ).

Potentiometric: The displacement of the spring-mass system is linked mechanically to a wiper arm, which moves along a potentiometer. The system can use gas, viscous, or magnetic damping to minimize acoustic noise caused by contact resistance of the wiper arm. Potentiometric accelerometers typically have a frequency range from zero to $20-60 \mathrm{~Hz}$, lower than most other accelerometers, depending on the stiffness of the spring, and have a high-level output signal.

Reluctive: Reluctive accelerometers use an inductance bridge to produce an output voltage signal proportional to the movement of the vibrating mass. The displacement of the vibration mass in inductance-bridge accelerometers causes the inductances of two coils to vary in opposing directions. The coils act as two arms of an inductance bridge, with resistors as the other two arms. The alternative current (AC) output voltage of the bridge varies with applied acceleration. A demodulator can be used to 
convert the AC signal to direct current (DC). An oscillator can be used to generate the required AC current when a DC power supply is used, as long as the frequency of the AC signal is far greater than that of the frequency of the acceleration.

Servo: In servo accelerometers, acceleration causes a vibrating mass "pendulum" to move. When motion is detected by a position-sensing device, a signal is produced that acts as the error signal in the closed-loop servo system. After the signal has been demodulated and amplified to remove the steady-state component, the signal is passed through a passive damping network and is applied to a torque coil located at the axis of rotation of the mass. The torque developed by the torque coil is proportional to the current applied, and counteracts the torque acting on the vibrating mass due to the acceleration, preventing further motion of the mass. Therefore, the current through the torque coil is proportional to acceleration. This device can also be used to measure angular acceleration as long as the vibrating mass is balanced. Servo accelerometers provide high accuracy and a high-level output though at a relatively high cost, and can be used for very low measuring ranges (well below $1 \mathrm{~g}$ ).

Strain gauge: Strain gauge accelerometers, often called "piezoresistive" accelerometers, use strain gauges acting as arms of a Wheatstone bridge to convert mechanical strain to a DC output voltage. The gauges are either mounted to the spring, or between the vibrating mass and the stationary frame. Strain gauge windings, which contribute to the spring action, are stressed (two in tension, two in compression), and a DC output voltage is generated by the four arms of the bridge that is proportional to the applied acceleration. These accelerometers can be made more sensitive with the use of semiconductor gauges and stiffer springs, yielding higher frequency response and 
output signal amplitude. And unlike other types of accelerometers, strain gauge accelerometers respond to steady-state accelerations.

Capacitive: A change in acceleration causes a change in the space between the moving and fixed electrodes of a capacitive accelerometer. The moving electrode is typically a diaphragm-supported vibrating mass or a flexure-supported, disk-shaped vibrating mass. The element can act as the capacitor in the inductor-capacitor (LC) or resistorcapacitor (RC) portion of an oscillator circuit. The resulting output frequency is proportional to the applied acceleration.

Vibrating element: In a vibrating element accelerometer, a very small displacement of the vibrating mass varies the tension of a tungsten wire in a permanent magnetic field. A current through the wire in the presence of the magnetic field causes the wire to vibrate at its resonant frequency (like a guitar string). The circuitry then outputs a frequency modulation (deviation from a center frequency) that is proportional to the applied acceleration. Although the precision of such a device is high, it is quite sensitive to temperature variations and is relatively expensive. 


\section{Appendix B - Example of Accelerometer and Data-Logger}

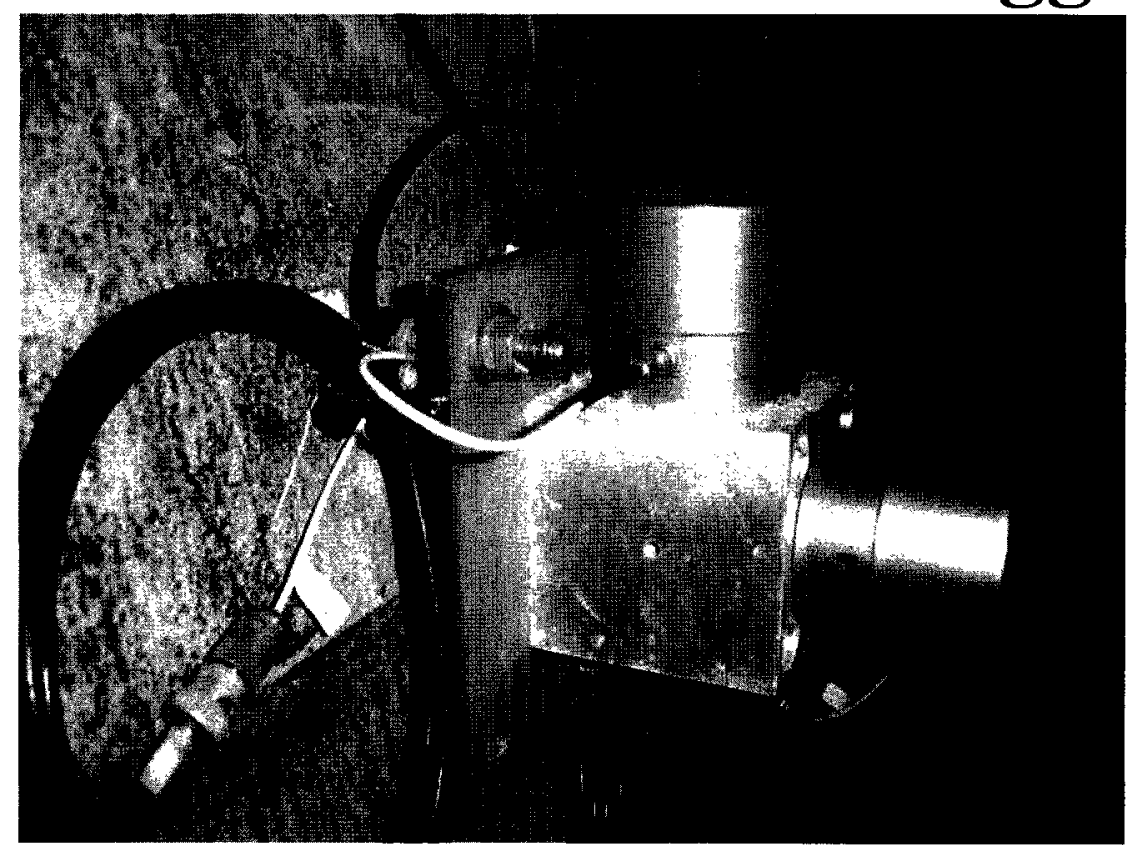

Example of two mounted PCB 393C accelerometers. Mounted at sensor location 14 and measuring vertical and transverse accelerations.

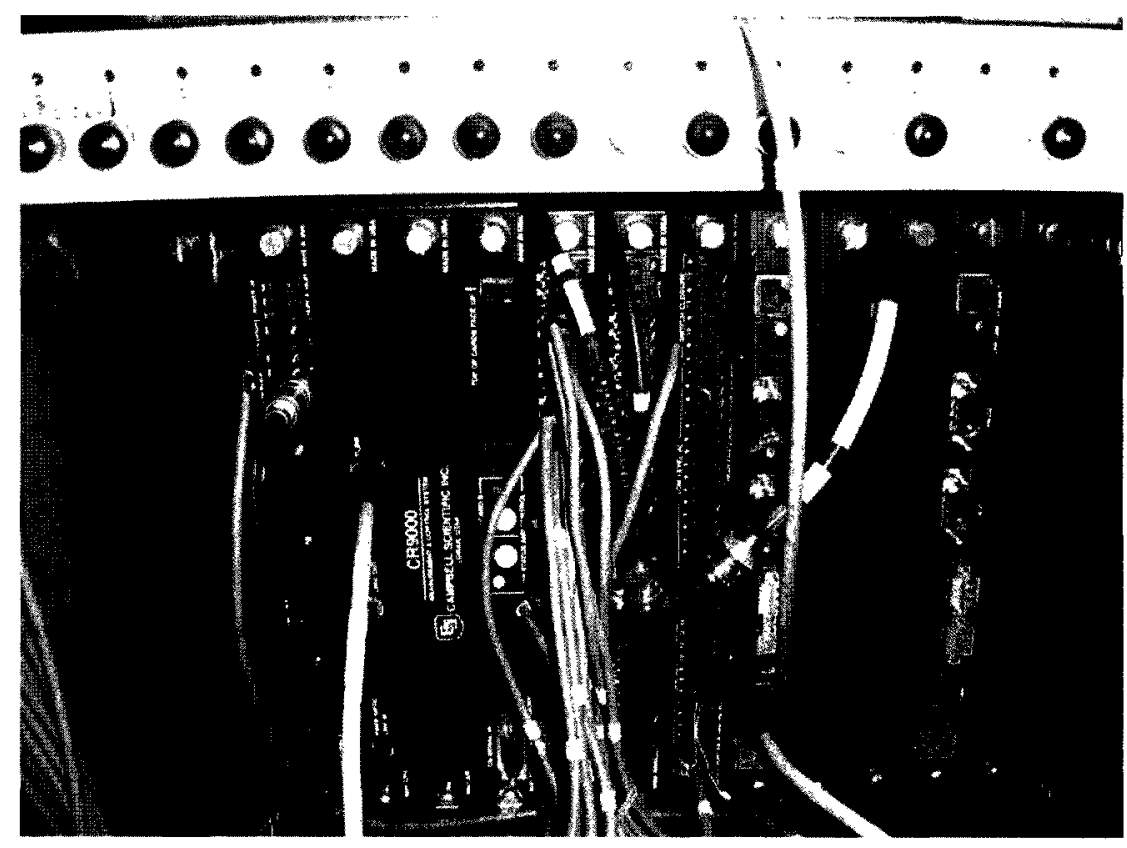

Example of CR9000 data-logger with signal conditioner. Shown is data-logger number 4 located inside bridge pier number 31 . 


\section{Appendix C - Processing Module Code Structure}

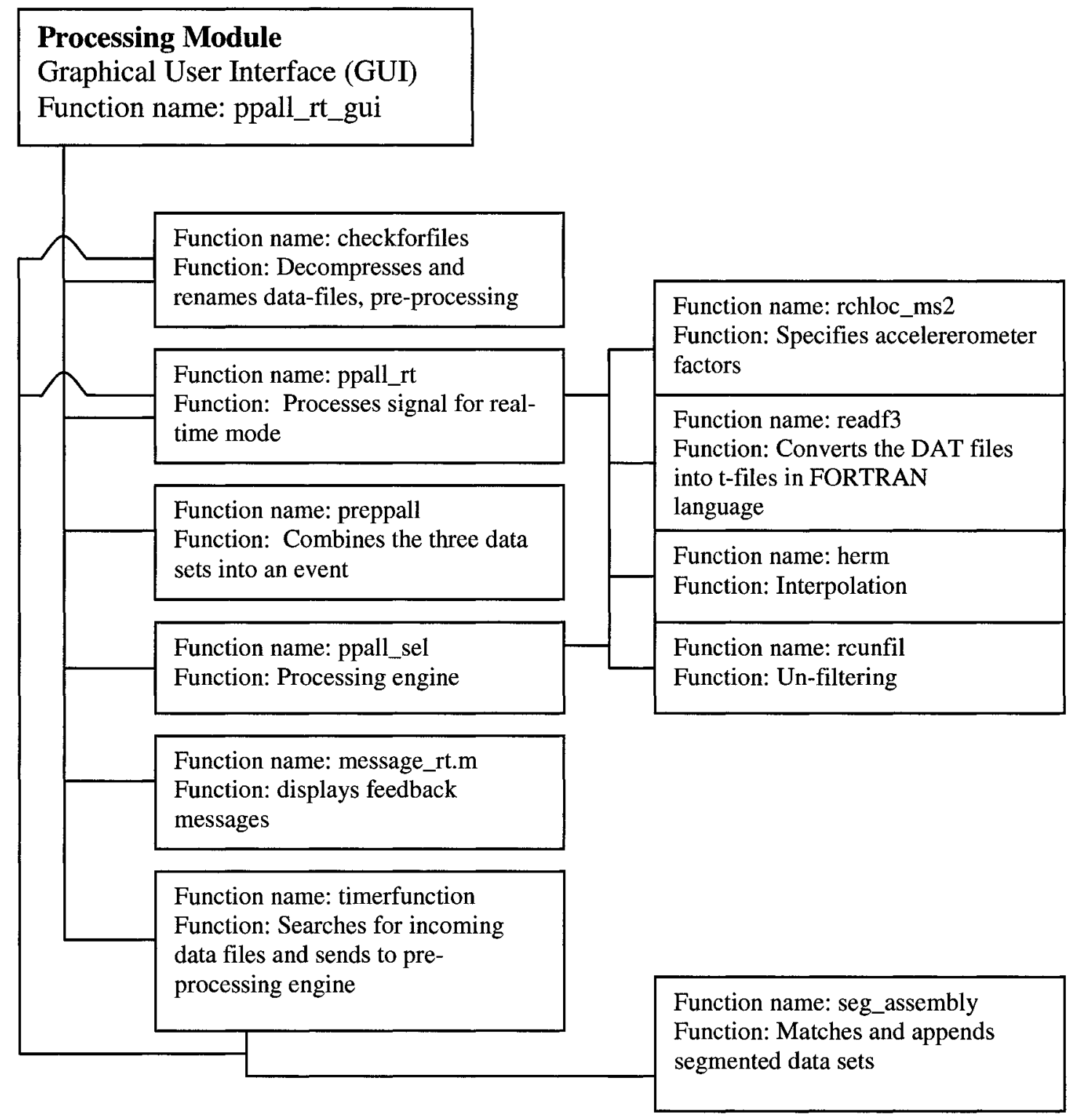




\section{Appendix D - Analysis Module Code Structure}

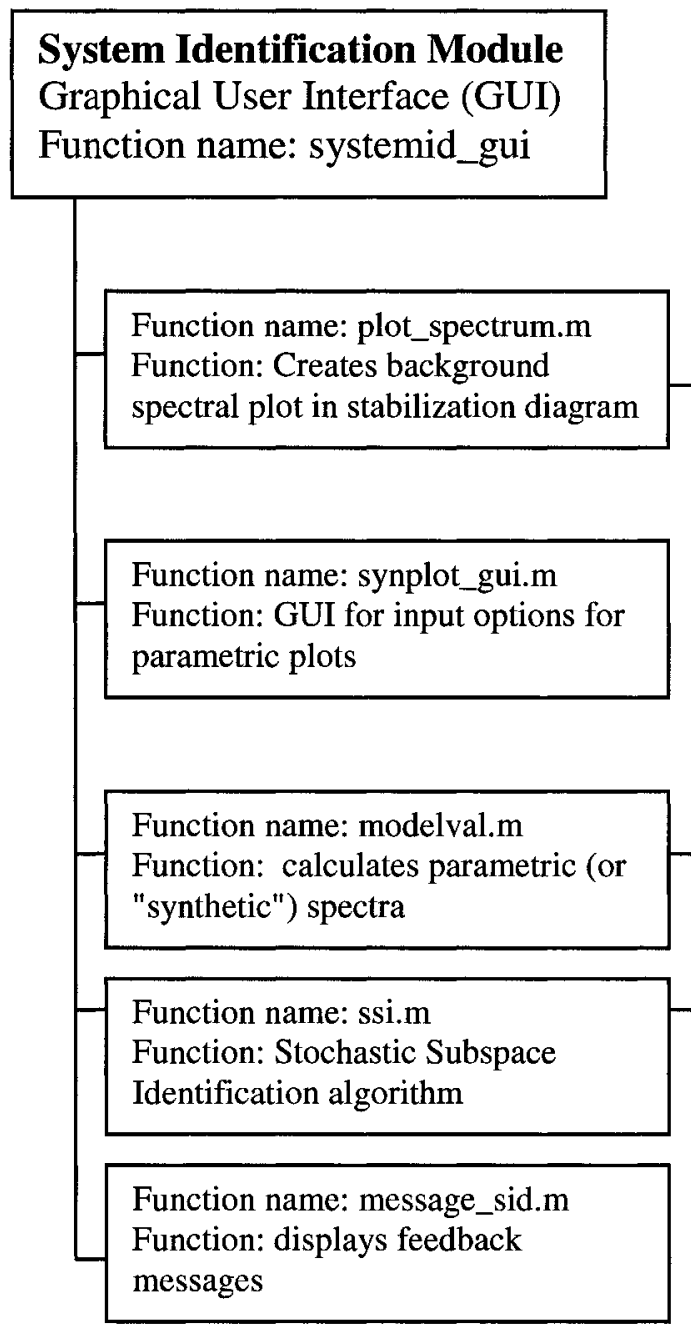

Function name: spec.m Function: Computes the averaged cross-power spectra

Function name: specplot.m Function: Plots cross-power spectra in active axes
Function name: powerspec.m Function: Cross power spectra computation

Function name: sumcp.m Function: calculates normalized sum of cross powers.

Function name: paramspec.m Function: computes the parametric spectrum matrix

Function name: ypf.m

Function: Assembles Ypast_ref and Yfuture matrices

Function name: toep.m

Function: Calculates the toeplitz matrix

Function name: part_svd.m

Function: takes a subset of $\mathrm{k}$ singular values

Function name: partifac.m

Function: Computes the reduced stochastic modal participation matrix

Function name: stab.m Function: plots appropriate characters in stabilization diagram

Function name: mac.m Function: Calculates modal assurance criteria for 2 mode-shapes 


\section{Appendix E - Visualization Modules Code Structure}

\section{Animation Module \\ Graphical User Interface (GUI) \\ Function name: bridge_animate_gui.m \\ Function name: animrt_timerfunction.m Function: runs the real-time animation of bridge responses.}

Function name: anim_funct.m Function: animation function for simple wire mesh bridge model.

Function name: animasth_funct.m Function: animation function for detailed fine meshed bridge model.

Function name: animasth_funct $2 . m$ Function: animation function for detailed bridge model.

Function name: anim_mode.m Function: animates extracted and analytical mode shapes on fine meshed bridge model

Function name: anim1_mode.m Function: animates extracted and analytical mode shapes on wire mesh bridge model

Function name: anim2_mode.m Function: animates extracted and analytical mode shapes on detailed bridge model

Function name: bridge.m Function: plots simple wire mesh bridge inside GUI.

Function name: bridge_asth.m Function: plots fine meshed bridge model inside GUI.

Function name: bridge_asth2.m Function: plots detailed bridge model inside GUI.

Function name: message.m

Function: displays feedback messages

\section{Data Display Module}

Graphical User Interface (GUI)

Function name: acc_plot_gui.m

Function name: message2.m

Function: displays feedback messages 